\title{
SEARCH FOR THE STANDARD MODEL HIGGS BOSON IN LEPTONS PLUS JETS FINAL STATES
}

\author{
Huong Thi Nguyen \\ Hanoi, Vietnam
}

M.S., Hanoi University of Education, 1994

\begin{abstract}
A Dissertation presented to the Graduate Faculty of the University of Virginia in Candidacy for the Degree of Doctor of Philosophy
\end{abstract}

Department of Physics

Unversity of Virginia

January, 2014 
To my parents 


\title{
ACKNOWLEDGMENTS
}

First and foremost, I gratefully acknowledge the continual guidance and support of my adviser, Professor Bob Hirosky. His dedication to research and pursuit of physics have been an invaluable source of inspiration and encouragement to us, his students.

I had the privilege of being on the teams of the ZHllbb and LNUJJ analysis groups in the D0 Collaboration and I would like to deeply thank all members of these two research groups. I will always remember the stimulating discussions and scientific atmosphere we shared with one another over the last four years.

Finally, I could not have achieved this without the support, encouragement and love from my friends and family, particularly my children, Chau and Minh.

\author{
Huong Nguyen \\ Charlottesville, Virginia
}

January, 2014 


\section{Contents}

1 Introduction 1

2 The Standard Model and Higgs Physics 4

2.1 The Standard Model of Particle Physics . . . . . . . . . . . . . . . . . 4

2.1 .1 Elementary particles . . . . . . . . . . . . . . . 5

2.1 .2 Interactions . . . . . . . . . . . . . . . . 7

2.1.3 The Higgs Mechanism . . . . . . . . . . . . . . . . . . . . . 15

2.1.4 Yukawa Interaction and Fermion Mass . . . . . . . . . . . . . . . . . 19

2.2 The Standard Model Higgs Physics . . . . . . . . . . . . . . . 23

2.2.1 Theoretical Constraints on the Higgs Boson Mass . . . . . . . . . . . 23

2.2 .2 Indirect Searches for the SM Higgs boson . . . . . . . . . . . . 26

2.2.3 Direct Searches for the SM Higgs Boson . . . . . . . . . . . . . . . . 28

2.3 Higgs Physics Beyond the Standard Model . . . . . . . . . . . . . . . . . 37

2.3.1 Fourth Generation of Fermions and Higgs Physics . . . . . . . . . 37

2.3.2 Fermiophobic Higgs Model . . . . . . . . . . . . . . . . . 37

2.4 Search for the SM Higgs Boson in Leptons plus Jets Final States . . . . . . 38

2.4.1 The SM Higgs Boson Signal in $\boldsymbol{Z} \boldsymbol{H} \rightarrow \boldsymbol{\ell} \boldsymbol{\ell} \boldsymbol{b} \boldsymbol{b}$ and $\boldsymbol{\ell} \boldsymbol{\nu} \boldsymbol{j} \boldsymbol{j}$ Analyses . . 38

2.4.2 Background in $\boldsymbol{Z} \boldsymbol{H} \rightarrow \boldsymbol{\ell} \boldsymbol{\ell} \boldsymbol{b} \boldsymbol{b}$ and $\boldsymbol{\ell} \boldsymbol{\nu} \boldsymbol{j} \boldsymbol{j}$ Analyses . . . . . . . . . 39

2.4.3 Analysis Procedure in $\boldsymbol{Z} \boldsymbol{H} \rightarrow \boldsymbol{\ell} \boldsymbol{\ell} \boldsymbol{b} \boldsymbol{b}$ and $\boldsymbol{\ell} \boldsymbol{\nu} \boldsymbol{j} \boldsymbol{j}$ Channels . . . . . . 43

3 The Experiment $\quad 46$

3.1 The Tevatron . . . . . . . . . . . . . . . . . . 46

3.2 The Dø Detector . . . . . . . . . . . . . . . . . . . 48

3.2.1 Coordinate System . . . . . . . . . . . . . . . . . . 49

3.2 .2 Central Tracking System . . . . . . . . . . . . . . . . . . . . . . . . . . . . . . . . . .

3.2 .3 Preshower Detectors . . . . . . . . . . . . . . . . 51

3.2 .4 Calorimeter System _. . . . . . . . . . . . . . . 52

3.2 .5 Muon System . . . . . . . . . . . . . . . . . . . . . . . . . . . . . . . 54

3.2 .6 Luminosity Monitor . . . . . . . . . . . . . . . . . . 57

3.2.7 Trigger and Data Acquisition Systems . . . . . . . . . . . 58 
4 Event Reconstruction $\quad 62$

4.1 The DØRECO Program . . . . . . . . . . . . . . . . . . 62

4.2 Particle Identification $\ldots \ldots \ldots \ldots$

4.2 .1 Electron Identification . . . . . . . . . . . . . . . . . 63

4.2 .2 Muon Identification _ . . . . . . . . . . . . . . . . 67

$4.2 .3 \quad$ Jet Identification . . . . . . . . . . . . . . . . . . . . . . . . . . . . 69

4.2.4 Missing Transverse Energy _. . . . . . . . . . . . . . . . . . . 79

$4.2 .5 \quad \boldsymbol{b}$-jet Identification . . . . . . . . . . . . . . . . . . . . 80

5 Signal Prediction and Background Estimation $\quad \mathbf{8 5}$

5.1 Data Samples . . . . . . . . . . . . . . . . . . . . . 85

5.2 Monte Carlo Generators . . . . . . . . . . . . . . . . 86

5.2 .1 PYTHIA ............................ 86

5.2 .2 ALPGEN . . . . . . . . . . . . . . . . . . 87

5.2 .3 SingleTop . . . . . . . . . . . . . . . . . . . 87

$5.2 .4 \mathrm{MCFM} \ldots \ldots \ldots \ldots \ldots \ldots$

5.2 .5 MLM Scheme . . . . . . . . . . . . . . . . . . 87

5.3 Detector Simulation . . . . . . . . . . . . . . . . . 88

5.4 Simulated Event Samples _ . . . . . . . . . . . . . . . . . . 88

5.4.1 Simulated signal processes . . . . . . . . . . . . . . . . 88

5.4 .2 Simulated Background processes . . . . . . . . . . . . . 90

5.5 Estimation of Multijet Background . . . . . . . . . . . . . . . . . 92

5.5.1 Multijet background modeling strategy . . . . . . . . . . . . 92

5.5 .2 Derivation of $P_{L T}^{M J} \ldots \ldots \ldots \ldots \ldots \ldots 4 \ldots \ldots \ldots$

5.5 .3 Derivation of $\epsilon_{L T}^{\ell} \ldots \ldots \ldots \ldots \ldots \ldots$

5.6 Simulated Sample Corrections . . . . . . . . . . . . . . . . 95

5.6.1 Reconstruction Efficiency Corrections . . . . . . . . . . . . . 96

5.6 .2 Trigger Correction . . . . . . . . . . . . . . . . . 97

5.6 .3 Luminosity Reweighting . . . . . . . . . . . . . . . . . . . . . . 97

5.6.4 Primary Vertex Reweighting . . . . . . . . . . . . . . . . 98

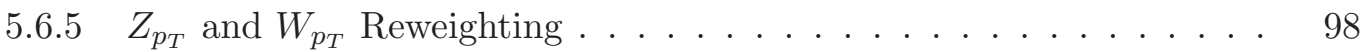

$5.6 .6 \quad \boldsymbol{V}+$ jets Reweighting . . . . . . . . . . . . . 98

6 Event Selections $\quad 104$

6.1 Event Selection in $\ell \nu j j$ Analysis . . . . . . . . . . . . . . . . . . 104

6.1.1 Online Event Selection . . . . . . . . . . . . . . . . . . . . . . . 104

6.1 .2 Offline Event Selection _ . . . . . . . . . . . . 106

6.1.3 Normalization at the Preselection . . . . . . . . . . . . . . . . 107

6.1.4 Classifying b-tag Samples . . . . . . . . . . . . . . . . . 108

6.1.5 Organizing the Search in $\ell \nu j j$ Final State . . . . . . . . . . 115 
6.2 Event Selection in $\boldsymbol{Z} \boldsymbol{H} \rightarrow \boldsymbol{\ell} \boldsymbol{\ell b} \boldsymbol{b}$ Analysis . . . . . . . . . . . . 115

6.2.1 Event Triggering . . . . . . . . . . . . . . . . . . 115

6.2 .2 Offline Event Selection ～. . . . . . . . . . . . . . . 116

6.2.3 Multijet Background and Normalization at the Preselection . . . . . 117

6.2.4 Classifying b-tag Samples . . . . . . . . . . . . . . . . . 118

7 Multivariate Signal Discriminants $\quad 121$

7.1 Mutivariate Analysis in $\boldsymbol{H} \rightarrow \boldsymbol{W} \boldsymbol{W} \rightarrow \boldsymbol{\mu \nu} \boldsymbol{j}$ Channel . . . . . . . . . . . 122

7.1.1 Random Forest of Decision Trees . . . . . . . . . . . . . . . . . 122

7.1.2 Optimizing Random Forest Classifiers . . . . . . . . . . . . . . 123

7.1.3 Radom Forest Discriminants . . . . . . . . . . . . . . . . . . . 133

$7.2 \quad$ MVA Analysis in $\boldsymbol{Z} \boldsymbol{H} \rightarrow \boldsymbol{\ell} \ell \boldsymbol{b} \boldsymbol{b}$ Search . . . . . . . . . . . . 136

7.2.1 Matrix Elements Discriminants . . . . . . . . . . . . . . . 136

7.2.2 Kinematic Fit and Random Forest Discriminants . . . . . . . . . . . 142

8 Systematic Uncertainties $\quad 146$

8.1 Systematic Uncertainties in $\boldsymbol{H} \rightarrow \boldsymbol{W} \boldsymbol{W} \rightarrow \boldsymbol{\mu} \boldsymbol{\nu} \boldsymbol{j}$ Analysis . . . . . . . . 147

8.1 .1 Flat Systematics Uncertainties . . . . . . . . . . . . . . . 147

8.1.2 Shape Systematics Uncertainties . . . . . . . . . . . . . . . . 147

8.2 Systematic Uncertainties in $\boldsymbol{Z} \boldsymbol{H} \rightarrow \boldsymbol{\ell} \ell \boldsymbol{b} \boldsymbol{b}$ Analysis . . . . . . . . . . 149

9 Results of the Higgs Boson Searches 151

$9.1 \mathbf{C L}_{\mathbf{S}}$ Method for Limit Calculation . . . . . . . . . . . . . . . . 151

9.1.1 Procedure for Limit-Setting . . . . . . . . . . . . . . . 151

9.1.2 Treatment of Uncertainties . . . . . . . . . . . . . . . . . 154

9.2 Results of the Higgs Boson Searches in $\ell \boldsymbol{\nu} j \boldsymbol{j}$ Analysis . . . . . . . . . . 156

9.2.1 Upper Limits on the SM Higgs Boson Production . . . . . . . . . . 156

9.2.2 Interpretation in Fourth Generation and Fermiophobic Higgs Boson Models . . . . . . . . . . . . . . . . . 160

9.3 Results of the SM Higgs Boson Searches in $\boldsymbol{Z} \boldsymbol{H} \rightarrow \boldsymbol{\ell} \boldsymbol{\ell} \boldsymbol{b} \boldsymbol{b}$ Analysis . . . . . . 166

9.3.1 Upper Limits on the SM Higgs Boson Production . . . . . . . . . . . 166

9.3.2 RF Technique Validation with Matrix Element Results . . . . . . . . 167

9.4 Combined Results from DØ and Tevatron Higgs Boson Searches . . . . . . 169

$\begin{array}{ll}10 \text { Conclusion } & 173\end{array}$ 


\section{Introduction}

What would have happened if all known elementary particles in the Universe were massless? No atoms would have formed, no planets would have developed and no life would have evolved. But why do some particles have mass (like the $Z$ boson) and some don't (like the photon)? How do the massive elementary particles gain their mass? Tackling these fundamental questions has been a major goal of particle physics for more than the last 50 years. Enormous effort from scientists and laboratories all over the world have been devoted to find solutions for these mass-related questions in the context of different physics models.

The Standard Model (SM) of particle physics is a mathematical model that codifies nearly all the properties and interactions of the fundamental constituents of visible matter in the Universe. Indeed, it is the most successful physics model in describing the results of myriads of accelerator experiments. The predictions of the Standard Model have been crowned with series of historical successes including the discoveries of neutral currents, the charm, bottom and top quarks, and the $W^{ \pm}$and $Z^{0}$ bosons. In order to answer the mass questions, an intriguing solution called the Higgs mechanism was incorporated and developed in the SM to originate the masses of $W^{ \pm}$and $Z^{0}$ gauge bosons through the process of spontaneous symmetry breaking. Based on the Higgs mechanism, the Standard Model further predicts the existence of a neutral scalar particle, the Higgs boson, and explains the origin of fundamental fermion masses through the interaction between fermion fields and the Higgs field. The Standard Model requires the Higgs boson be the unique source of mass for all quarks, charged leptons, and vector gauge bosons, and it implies that the couplings of the Higgs boson to those fundamental massive particles are precisely in the ratio of their masses. However, the mass of the SM Higgs boson itself remains an unknown parameter.

The Higgs mechanism in the Standard Model insists that the SM Higgs boson is the only physical manifestation of the origin of mass, therefore, the key to confirming the Higgs mechanism is verifying the existence of the SM Higgs boson. Indirect and direct searches for the SM Higgs boson over last three decades provide stringent constraints on its mass. Based on requirements of consistency in the SM framework, precision electroweak data indirectly constrain the allowed mass of a SM Higgs boson to $M_{H}<152 \mathrm{GeV}$ [1] 
at the $95 \%$ confidence level (C.L.). Direct searches for the production of a SM Higgs boson at the ALEPH, DELPHI, L3, and OPAL experiments at the LEP $e^{+} e^{-}$collider [2], the CDF and D0 experiments at the Tevatron $p \bar{p}$ collider [3, 4], and the ATLAS [5] and CMS [6] experiments at the LHC $p p$ collider limit the mass of the SM Higgs boson to $122 \mathrm{GeV}<M_{H}<127 \mathrm{GeV}$ at $95 \%$ C.L.

The ATLAS and CMS Collaborations have recently discovered a Higgs boson with a mass of $\sim 126 \mathrm{GeV}[7,8]$. The $\mathrm{CDF}$ and D0 Collaborations have reported combined evidence for a particle, with a mass consistent with that of the Higgs boson observed at the $p p$ collider, produced in association with a $W$ or $Z$ boson and decaying to a bottom-antibottom quark pair [9]. Most of the resolving power for the SM Higgs boson searches at the ATLAS and CMS Collaborations comes from the bosonic final states where the Higgs boson decays to $\gamma \gamma, Z Z$, and $W^{+} W^{-}$, while much of the signal sensitivity of the search for the SM Higgs boson at the Tevatron in the low mass region near $126 \mathrm{GeV}$ is due to the fermionic decay mode $H \rightarrow b \bar{b}$. Therefore, the Tevatron results continue to provide information to help unravel the nature of the discovered boson.

This dissertation presents searches for SM Higgs boson production in final states containing leptons (where the leptons can be charged leptons $\ell$ or neutrinos $\nu$ ) and hadronic jets $(j)$ initiated by quarks. We utilize a data set corresponding to $9.7 \mathrm{fb}^{-1}$ of $p \bar{p}$ collisions at the central-of-mass energy of $\sqrt{s}=1.96 \mathrm{TeV}$ collected by the $\mathrm{D} \varnothing$ experiment. The searches are carried out in two independent analyses accounting for different signal topologies. The $\ell \nu j j$ analysis comprises searches for three main signal processes, $W H \rightarrow \ell \nu b b$, $H \rightarrow W W \rightarrow \ell \nu j j$, and $W H \rightarrow W W W \rightarrow \ell \nu j j j j$, where the SM Higgs boson is produced either in association with a $W$ boson or through the gluon-gluon fusion process, and then decays to either a bottom and anti-bottom quark $b \bar{b}$ pair or a vector boson $W^{+}$and $W^{-}$ pair. The $Z H \rightarrow \ell \ell b b$ analysis performs the search for the SM Higgs boson produced in association with a $Z$ boson and subsequently decaying to bottom and anti-bottom quark pair. In addition to the interpretation in the context of the Standard Model, the searches in the $\ell \nu j j$ analysis are also interpreted in models containing a fourth generation of fermions, and models with a fermiophobic Higgs boson. Results of the searches for the Higgs boson in the $\ell \nu j j$ and the $Z H \rightarrow \ell \ell b b$ analyses have been published in Refs. [10] and [11]. Search results obtained from these two analyses are also combined with those from other channels at the D $\varnothing$ and CDF experiments and the combined results published in Refs. [12] and [13].

The dissertation is structured in ten chapters. Chapter 2 introduces the Standard Model of particles physics, provides an overview of the physics of the Higgs sector in the SM framework as well as in frameworks extended from the SM, and outlines the $\ell \nu j j$ and $Z H \rightarrow \ell \ell b b$ analyses. Descriptions of the fundamental particles and interactions in the SM framework and of the process of incorporating the Higgs mechanism in the SM to originate the masses for the weak gauge bosons and fundamental fermions as well as to give a rise to the Higgs boson are given in Sec. 2.1. Phenomenological studies and current theoretical and experimental constraints on the mass of the Higgs boson in the SM, as well as in extended 
models containing fourth generation fermions or a fermiophobic Higgs boson, are presented in Secs. 2.2 and 2.3. A brief introduction to the procedures employed in the $\ell \nu j j$ and $Z H \rightarrow \ell \ell b b$ analyses and a review of the main contributions to each analysis step from the author of this thesis are given in the last section of Chapter 2 (Sec. 2.4).

To explore the particle world and seek evidence for the SM Higgs boson, we first have to produce powerful particle collisions and build an intricate detector to observe the outcome of the collisions with high precision. Chapter 3 briefly describes the process of producing $p \bar{p}$ collisions at the Fermilab Tevatron Collider and outlines the characteristics and performance of the $\mathrm{D} \varnothing$ detector used to collect the data analyzed in this thesis. An overview of programs used to translate data recorded by the detector into properties of physics events along with techniques and algorithms used for reconstructing and identifying physics objects from $p \bar{p}$ collision events are described in Chapter 4.

Various SM processes can mimic the signatures of the targeted SM Higgs boson signals and contribute to the background of the corresponding analysis. Chapter 5 discusses the common methods of simulating signal and background processes based on the SM prediction and estimating background compositions of the data. Chapter 6 describes the event selections applied in each of the $\ell \nu j j$ and $Z H \rightarrow \ell \ell b b$ analyses and discusses the orgnization of selected events further into subchannels based on charged lepton flavor, jet multiplicity, and the number and quality of candidate $b$ quark jets in the final states to maximize the sensitivity for each particular Higgs boson production and decay mode.

Despite the imposing of selection requirements significantly enhancing signal purity in data, the search for the Higgs boson is still challenging due to the presence of prodigious background at the Tevatron. To improve the power of differentiating signal and background events mixed in a data sample, multivariate analysis (MVA) techniques are employed to incorporate signal-to-background discriminating information from individual event kinematic variables into a single MVA discriminant. Details of optimizing the performance of the MVAs in each analyses are described in Chapter 7. An overview of the most important systematic uncertainties arising from various sources, such as reconstruction of physics objects, calibration of measured quantities and simulations, and their impact on the normalization and shape of the MVA discriminant are provided in Chapter 8.

The MVA discriminant distributions, together with the impact of associated uncertainties in their construction, are used as inputs to a procedure of statistical calculations to extract the upper limits on the Higgs boson production cross section multiplied by the corresponding branching fraction. Chapter 9 describes the limit setting process and the treatment of systematic uncertainties and presents our search results for the SM Higgs boson as well as two interpretations beyond the SM.

Finally, concluding remarks on the completed analyses and the Higgs boson results from our individual analyses as well as from the full Tevatron combination are presented in Chapter 10. The dissertation is closed by a short discussion of new directions for physics in the future years Anno Higgsi $\mathrm{AH}>0$. 
2

\section{The Standard Model and Higgs Physics}

\subsection{The Standard Model of Particle Physics}

The Standard Model (SM) of particle physics is a well established gauge theory based on principles of symmetry. It describes basically all fundamental particles and interactions that we have been able to probe in the nature. Among the four known fundamental interactions, only the gravitational interaction is not included in the framework of the SM, because quantum gravity effects are very weak at technological accesible energy scale and therefore we have no experimental input about quantum gravity. The strong, the weak and the electromagnetic interactions in the SM model are determined by the gauge symmetry group:

$$
\mathrm{SU}(3)_{C} \otimes \mathrm{SU}(2)_{L} \otimes \mathrm{U}(1)_{Y}
$$

where $\mathrm{C}$ denotes the color charge, $\mathrm{L}$ implies that $\mathrm{SU}(2)$ acts on the left-handed fields, and $\mathrm{Y}$ indicates the weak hypercharge which relates to the electric charge and the weak isospin. The strong force corresponds to the $\mathrm{SU}(3)_{C}$ group, while the electromagnetic and the weak forces are unified in the $\mathrm{SU}(2)_{L} \otimes \mathrm{U}(1)_{Y}$ symmetry group.

The elementary particles in the Standard Model are classified into two groups, the fermions with spin $\frac{1}{2}$ and the gauge bosons with spin 1 . While the elementary fermion particles interact through the gauge fields and serve as the sources, the gauge bosons are the quanta associated with the gauge fields mediating the interactions between fermions. The gauge bosons mediating the electromagnetic and strong forces (photon and gluons) are massless, but the weak force carriers ( $W^{ \pm}$and $Z^{0}$ bosons) have masses of the order of $100 \mathrm{GeV}$.

The deep connections between symmetries and physics are mathematically encoded in the structure of the Lagrangian of a physics system by demanding the Lagrangian to be invariant under gauge transformations operating on the system. Inserting explicitly 
the mass terms for the gauge fields into the Lagrangian destroys the gauge invariance and renders the theory meaningless. Therefore, an intriguing mechanism, called the Higgs mechanism, was developed to originate the masses of $W^{ \pm}$and $Z^{0}$ gauge bosons through the process of spontaneous symmetry breaking. Based on the Higgs mechanism, the Standard Model further predicts the existence of a neutral scalar particle, the Higgs boson, and explains the origin of fermion masses through the interaction between fermion fields and the Higgs field.

In the following sections, the fundamental particles and interactions will be described first, and then the mechanism of originating the masses for the weak gauge bosons and the elementary fermions within the Standard Model framework will be presented.

\subsubsection{Elementary particles}

\section{Fermions}

The elementary fermion particles in the Standard Model are separated into two groups, quarks and leptons. The quarks are confined in compound systems (hadrons) by the strong interaction. Leptons, on the other hand, do not participate in the strong interaction.

Six types of leptons have been observed and incorporated in the Standard Model: electron $\left(e^{-}\right)$, muon $\left(\mu^{-}\right)$, tau $\left(\tau^{-}\right)$, electron neutrino $\left(\nu_{e}\right)$, muon neutrino $\left(\nu_{\mu}\right)$ and tau neutrino $\left(\nu_{\tau}\right)$. Three charged leptons $\left(e^{-}, \mu^{-}, \tau^{-}\right)$are massive and experience both weak and electromagnetic interactions. While the electron is stable, the other two charged leptons (muon and tau) have finite lifetimes and decay via the weak interaction. The fact that neutrinos participate exclusively in the weak interaction and do not decay makes experimental investigations of neutrinos very challenging. Due to the field content (there are no scalar triplets or fields that are singlet under the gauge group $\left.N_{R}(1,1)_{0}\right)$ and the renormalizable requirement of the theory, the Standard Model implies that neutrinos are massless. However, recent searches for neutrino flavor oscillations strongly indicate that neutrinos are massive. Therefore, the Standard Model needs to be extended in this respect.

The Standard Model considers six different flavors of quarks: up $(u)$, down $(d)$, charm $(c)$, strange $(s)$, top $(t)$, and bottom $(b)$. Each quark flavor has three quark color states (red, green and blue) and carries fractional electric charge ${ }^{1}$. The up-type quarks, $(u, c, t)$ have electric charge of $q=\frac{2}{3}$ and the down-type quarks $(d, s, b)$ carry electric charge of $q=-\frac{1}{3}$. As charged leptons, quarks interact through the electromagnetic interaction and the weak interaction. But by virtue of carrying the color charge, quarks also participate in the strong interaction. For an isolated system, the strong and electromagnetic interactions conserve the number of different flavors of quarks, but the weak interaction can change quark flavor. Quarks are confined in bound states of hadrons and no isolated quark has been observed in nature so far.

\footnotetext{
${ }^{1}$ Electric charge is in the units where the electron charge is $q=-1$.
} 
Within the Standard Model, leptons and quarks are organized in three generations as in Table 2.1. Each generation is a group of one charged lepton, a corresponding neutrino, one up type quark and one down type quark. The only distinction between generations is the difference in masses of particles. Each lepton or quark of a higher generation has greater mass than that of the corresponding lepton or quark of the lower generation. Having the same number of generations for quarks and lepton is a result of the anomaly cancellation conditions [14], but the reason for postulating exactly three generations in the SM is an open question for particle physics.

\begin{tabular}{||c|c|c|c|c||}
\hline \hline Generation & Symbol & Mass (MeV) & Charge $(e)$ & Interaction \\
\hline \hline \multirow{3}{*}{ First } & $e$ & 0.511 & -1 & EM, Weak \\
& $\nu_{e}$ & $<2 \times 10^{-6}$ & 0 & Weak \\
& $u$ & $2.3_{-0.5}^{+0.7}$ & $+\frac{2}{3}$ & EM, Weak, Strong \\
& $d$ & $4.8_{-0.3}^{+0.5}$ & $-\frac{1}{3}$ & EM, Weak, Strong \\
\hline \hline \multirow{3}{*}{ Second } & $\mu$ & 105.7 & -1 & EM, Weak \\
& $\nu_{\mu}$ & $<0.19$ & 0 & Weak \\
& $c$ & $1.275 \pm 0.025 \times 10^{3}$ & $+\frac{2}{3}$ & EM, Weak, Strong \\
& $s$ & $95 \pm 5$ & $-\frac{1}{3}$ & EM, Weak, Strong \\
\hline \hline \multirow{3}{*}{ Third } & $\tau$ & $1176.86 \pm 0.16$ & -1 & EM, Weak \\
& $\nu_{\tau}$ & $<18.2$ & 0 & Weak \\
& $t$ & $173.07 \pm 0.89 \times 10^{3}$ & $+\frac{2}{3}$ & EM, Weak, Strong \\
& $b$ & $4.18 \pm 0.03 \times 10^{3}$ & $-\frac{1}{3}$ & EM, Weak, Strong \\
\hline \hline
\end{tabular}

Table 2.1: Properties of quarks and leptons.

Each lepton or quark has an associated antiparticle. The antiparticles have the same mass and undergo the same interactions as corresponding particles, but have the reversed signs for some quantum numbers, such as electric charge, baryon number and lepton number. Quarks, leptons, antiquarks and antileptons together make a complete list of 24 elementary fermions in the Standard Model.

\section{Gauge bosons}

The gauge fields appear in the SM as a consequence of the invariance of the Lagrangian under local symmetry transformations. Quanta of those gauge fields are spin-1 particles, gauge bosons, which mediate the interactions between fermions.

The photon $(\gamma)$, the quanta of the electromagnetic interaction field between electrically charged fermions, has no electric charge and zero mass. Since the photon is massless, the associated electromagnetic force is a long range force.

The force carriers of the weak interaction are the charged $W^{+}$and $W^{-}$bosons and the neutral $Z$ boson that were discovered at CERN in 1983. Since the $W^{ \pm}$and $Z^{0}$ bosons carry mass $\left(M_{W} \approx 80.4 \mathrm{GeV}, M_{Z} \approx 91.2 \mathrm{GeV}\right)$, the weak force has a short range of $\approx 10^{-3} \mathrm{fm}$. At low energies, the weak interaction is much weaker than the EM interaction and the 
strong interaction. However, its unique features of maximally violating charge conjugation and parity and not to conserve flavor enabled the discovery of the weak force, long before the advent of high energy accelerators $[15,16]$.

\begin{tabular}{||c|c|c|c|c||}
\hline \hline Symbol & Mass $(\mathrm{MeV})$ & Interaction & Interact range & Interact with \\
\hline$\gamma$ & 0 & EM & Infinite & $\begin{array}{c}\text { charged leptons } \\
\text { quarks }\end{array}$ \\
\hline$W^{ \pm}$ & 80.4 & Weak & $10^{-18} m$ & quarks, leptons \\
$Z$ & 91.2 & & & quarks, gluons \\
\hline$g_{i}$ & 0 & Strong & $10^{-15} m$ & \\
\hline$(i=1, \ldots, 8)$ & & & & \\
\hline \hline
\end{tabular}

Table 2.2: The SM gauge bosons.

The quanta of the strong interaction field, the gluons $(g)$, mediate the strong force between color charged particles. The gluons are massless and electrically neutral as the photon, but they have eight different color charge combinations. Since gluons themselves carry the color charge and can directly interact with one another, the strong force between colored objects becomes larger when the objects are further apart and the strong interaction is confining. The properties of the gauge bosons and the interactions they associate with are summarized in Table 2.2 .

\subsubsection{Interactions}

One of the most profound insights in the Standard Model is that the interactions between fundamental particles can be dictated by the local gauge symmetries. In the simplest example, the requirement of the Lagrangian for a single fermion being invariant under the $U(1)_{E M}$ gauge transformation defines the electromagnetic interaction. The weak interaction as well as the unification of the weak interaction and the electromagnetic interaction are introduced by demanding the invariance of the Lagrangian under transformation of the $S U(2)_{L} \otimes U(1)_{Y}$ gauge group. Following similar procedure in Quantum Electrodynamics (QED), the strong interaction appears in the Standard Model by enforcing the Lagrangian of a single quark to be invariant under the $S U(3)_{C}$ gauge transformation. Finally, invoking gauge invariance under the transformations of the gauge group made by product of three sets of gauge transformations $S U(3)_{C} \otimes \mathrm{SU}(2)_{L} \otimes \mathrm{U}(1)_{Y}$ ensures that all three interactions (strong, weak and electromagnetic) are described in the Standard Model.

\section{Electromagnetic interaction : $\mathrm{U}(1)_{\mathrm{EM}}$}

We first consider QED, where quarks and leptons are represented by Dirac fields which are functions of the continuous space and time coordinates $x_{\mu}$. The most general renormalizable 
Lagrangian for a single Dirac fermion field has the following structure:

$$
\mathcal{L}=\bar{\psi}\left(i \gamma^{\mu} \partial_{\mu}-m\right) \psi
$$

Under the $U(1)_{E M}$ gauge group, the fermion field transforms as:

$$
\psi \rightarrow \psi^{\prime}=e^{i \alpha(x) Q} \psi
$$

where the charge operator Q (with eigenvalue of -1 for electron) acts as the generator of $U(1)_{E M}$ group.

Insisting the Lagrangian in Eqn. (2.2) to be invariant under the $U(1)_{E M}$ gauge transformation (Eqn. (2.3)) leads to the existence of the gauge field $A_{\mu}$. This process is mathematically done by replacing the $\partial_{\mu}$ in the Eqn. (2.2) by the covariant derivative:

$$
\partial_{\mu} \rightarrow D_{\mu}=\partial_{\mu}+i e Q A_{\mu}
$$

where $A_{\mu}$ transforms as:

$$
A_{\mu} \rightarrow A_{\mu}+\frac{1}{e} \partial_{\mu} \alpha
$$

Using the covariant derivative, the invariant Lagrangian can be expressed as:

$$
\mathcal{L}=\underbrace{\bar{\psi}\left(i \gamma^{\mu} \partial_{\mu}-m\right) \psi}_{\text {Free fermion }}-\underbrace{e \bar{\psi} \gamma^{\mu} Q \psi A_{\mu}}_{\text {Interaction }} .
$$

The second term in Eqn. (2.6) shows the electromagnetic interaction of the $A_{\mu}$ field and the fermion with coupling constant $e$.

Regarding $A_{\mu}$ in the interaction term as the physical field of a massless photon, we do not need to consider the mass term $\frac{1}{2} m^{2} A_{\mu} A^{\mu}$ of the gauge field. However, to describe the propagation of the gauge field, we must consider the dynamical contribution to the Lagarangian of the $A_{\mu}$ field. Defining the field strength tensor of gauge field $A_{\mu}$ by

$$
F_{\mu \nu}=\partial_{\mu} A_{\nu}-\partial_{\nu} A_{\mu}
$$

and taking a gauge invariant term corresponding to kinetic energy of the $A_{\mu}$ field to be $-\frac{1}{4} F_{\mu \nu} F^{\mu \nu}$, we obtain the Lagrangian of QED:

$$
\mathcal{L}_{E M}=\underbrace{\bar{\psi}\left(i \gamma^{\mu} \partial_{\mu}-m\right) \psi}_{\text {Free fermion }}-\underbrace{e \bar{\psi} \gamma^{\mu} Q \psi A_{\mu}}_{\text {Interaction }}-\underbrace{\frac{1}{4} F_{\mu \nu} F^{\mu \nu}}_{\text {Kinetic energy of } A_{\mu}} .
$$


Electroweak interaction : $\mathbf{S U}(2)_{\mathbf{L}} \otimes \mathbf{U}(1)_{\mathbf{Y}}$

In the chiral presentation, the four-component Dirac fields of quarks and leptons can be written as a pair of two-component fields:

$$
\psi=\left(\begin{array}{c}
\psi_{L} \\
\mathbf{0}
\end{array}\right)+\left(\begin{array}{c}
\mathbf{0} \\
\psi_{R}
\end{array}\right)
$$

where $\psi_{L}$ and $\psi_{R}$ are left-handed and right-handed fields which are projected out the Dirac field by the chirality operator $\gamma^{5}$ :

$$
\left(\begin{array}{c}
\psi_{L} \\
\mathbf{0}
\end{array}\right)=\frac{1}{2}\left(\mathbf{I}-\gamma^{\mathbf{5}}\right) \psi, \quad\left(\begin{array}{c}
\mathbf{0} \\
\psi_{R}
\end{array}\right)=\frac{\mathbf{1}}{\mathbf{2}}\left(\mathbf{I}+\gamma^{\mathbf{5}}\right) \psi
$$

A pair of left-handed charged lepton and the corresponding left-handed neutrino fields and a pair of left-handed up-type quark and left-handed down-type quark fields from each generation form doublets $\Psi_{L}$, while the right-handed fermion fields forming $\operatorname{singlets} \Psi_{R}$ :

$$
\begin{array}{cccccc}
\mathbf{\Psi}_{\mathbf{L}}:\left(\begin{array}{c}
\nu_{e} \\
e
\end{array}\right)_{L} \quad\left(\begin{array}{c}
\nu_{\mu} \\
\mu
\end{array}\right)_{L} \quad\left(\begin{array}{c}
\nu_{\tau} \\
\tau
\end{array}\right)_{L}\left(\begin{array}{l}
u \\
d
\end{array}\right)_{L}\left(\begin{array}{c}
c \\
s
\end{array}\right)_{L}\left(\begin{array}{l}
t \\
b
\end{array}\right)_{L} \\
\Psi_{\mathbf{R}}:=e_{R} & \mu_{R} & \tau_{R} & d_{R} & s_{R} & b_{R} \\
& & & u_{R} & c_{R} & t_{R}
\end{array}
$$

When the Standard Model was constructed, there was no experimental evidence for the existence of the right-handed neutrino, therefore, the right-handed neutrino fields were ignored. Today, however, we consider extensions of the Standard Model where such fields are included.

Since the group $S U(2)_{L}$ in the electroweak gauge symmetry $S U(2)_{L} \otimes U(1)_{Y}$ acts only on left-handed fields, the $S U(2)_{L} \otimes U(1)_{Y}$ transformations of the left-handed doublets and right-handed singlet are:

$$
\Psi_{L} \rightarrow \Psi_{L}^{\prime}=e^{i \boldsymbol{\alpha}(x) \cdot \mathbf{T}+i \beta(x) Y} \Psi_{L}, \quad \Psi_{R} \rightarrow \Psi_{R}^{\prime}=e^{i \beta(x) Y} \Psi_{R}
$$

In Eqn. $(2.14), \beta(x)$ and three components of the vector $\boldsymbol{\alpha}(x)=\left(\alpha_{1}(x), \alpha_{2}(x), \alpha_{3}(x)\right)$ are real parameters. The operator $Y$ and three components of isospin vector operator $\mathbf{T}=\left(T^{1}, T^{2}, T^{3}\right)$ are generators of the $U(1)_{Y}$ and $S U(2)_{L}$ groups, respectively.

The form of the generators depends on the representation of the field. In the fundamental representation, the generators of $S U(2)_{L}$ are $2 \times 2$ matrixes and written as $T^{a}=\frac{\tau^{a}}{2}(a=$ $1,2,3)$, where the $\tau^{a}$ are identical to the Pauli spin matrixes:

$$
\tau^{1}=\left(\begin{array}{cc}
0 & 1 \\
1 & 0
\end{array}\right) \quad \tau^{2}=\left(\begin{array}{cc}
0 & -i \\
i & 0
\end{array}\right) \quad \tau^{3}=\left(\begin{array}{cc}
1 & 0 \\
0 & -1
\end{array}\right)
$$


The generator of the $U(1)_{Y}$ group, the weak hyperchage operator $Y$, is defined as a linear combination of the electromagnetic charge operator $Q$ and the third generator $T^{3}$ of $S U(2)_{L}$ :

$$
Y=2\left(Q-T^{3}\right)
$$

The week isospin $(T)$ and hypercharge quantum numbers $(Y)$ of left-handed and right handed fundamental fermion fields of the first generation are summarized in Table 2.3.

\begin{tabular}{||c|c|c|c|c|c|c|c||}
\hline \hline Field & $\mathbf{e}_{\mathbf{L}}$ & $\boldsymbol{\nu}_{e L}$ & $\mathbf{u}_{\mathbf{L}}$ & $\mathbf{d}_{\mathbf{L}}$ & $\mathbf{e}_{\mathbf{R}}$ & $\mathbf{u}_{\mathbf{R}}$ & $\mathbf{d}_{\mathbf{R}}$ \\
\hline Weak Isospin T & $\frac{1}{2}$ & $\frac{1}{2}$ & $\frac{1}{2}$ & $\frac{1}{2}$ & 0 & 0 & 0 \\
\hline Hypercharge $\mathbf{Y}$ & -1 & -1 & $\frac{1}{3}$ & $\frac{1}{3}$ & -2 & $\frac{4}{3}$ & $-\frac{2}{3}$ \\
\hline \hline
\end{tabular}

Table 2.3: Weak Isospin and Hypercharge of Leptons and Quarks.

Consider the Lagrangian for a set of massless Dirac fermion fields:

$$
\mathcal{L}=\bar{\Psi}_{L} i \gamma^{\mu} \partial_{\mu} \Psi_{L}+\bar{\Psi}_{R} i \gamma^{\mu} \partial_{\mu} \Psi_{R}
$$

Demanding the Lagrangian of Eqn. (2.17) to be invariant under the gauge transformation of $S U(2)_{L} \otimes U(1)_{Y}$ symmetry (Eqn. (2.14) forces us to introduce four gauge vector fields, $\mathbf{W}_{\mu}=\left(W_{\mu}^{1}, W_{\mu}^{2}, W_{\mu}^{3}\right)$ and $B_{\mu}$, and replace the partial derivative by the covariant derivative:

$$
\partial_{\mu} \rightarrow D_{\mu}=\partial_{\mu}+i g W_{\mu}^{a} T^{a}+i \frac{g^{\prime}}{2} B_{\mu} Y
$$

where the three gauge fields $W_{\mu}^{a}(\mathrm{a}=1,2,3)$ are associated with the $S U(2)_{L}$ group and the $B_{\mu}$ gauge field is associated with the $U(1)_{Y}$ group, $g$ and $g^{\prime}$ are gauge coupling constants to the $W_{\mu}^{a}$ fields and $B_{\mu}$ fields. The gauge fields transform as:

$$
\begin{aligned}
B_{\mu} & \rightarrow B_{\mu}-\frac{1}{g^{\prime}} \partial_{\mu} \beta \\
W_{\mu}^{a} & \rightarrow W_{\mu}^{a}-\frac{1}{g} \partial_{\mu} \alpha^{a}-\epsilon_{a b c} \alpha^{b} W_{\mu}^{c},
\end{aligned}
$$

where $\epsilon_{a b c}$ is structure constant of the $S U(2)$ group $\left[T^{a}, T^{b}\right]=i \epsilon_{a b c} T^{c}$. The field strength tensors of the $B_{\mu}$ and $W_{\mu}^{a}$ gauge fields are defined by:

$$
\begin{gathered}
B_{\mu \nu}=\partial_{\mu} B_{\nu}-\partial_{\nu} B_{\mu} \\
W_{\mu \nu}^{a}=\partial_{\mu} W_{\nu}^{a}-\partial_{\nu} W_{\mu}^{a}-g \epsilon_{a b c} W_{\mu}^{b} W_{\nu}^{c} .
\end{gathered}
$$


The last term in Eqn. (2.20) arrises from non-Abelian nature of the group $S U(2)$ when generators $T^{i}(\mathrm{i}=1,2,3)$ do not commute with each other. We then take the dynamical contribution to the Lagrangian associated with $B_{\mu}$ and $W_{\mu}^{a}$ gauge fields to be:

$$
\mathcal{L}_{\text {gauge }}=-\frac{1}{4} B_{\mu \nu} B^{\mu \nu}-\frac{1}{4} W_{\mu \nu}^{a} W^{a \mu \nu}
$$

Replacing the partial derivative $\partial_{\mu}$ in Eqn. (2.17) with the covariant derivative of Eqn. (2.18) and considering the contribution to the Lagrangian of the gauge sector (Eqn. (2.21)) lead to the electroweak Lagrangian first developed by Glashow in 1961:

$$
\begin{array}{r}
\mathcal{L}_{E W}=\underbrace{\bar{\Psi}_{L} i \gamma^{\mu} \partial_{\mu} \Psi_{L}+\bar{\Psi}_{R} i \gamma^{\mu} \partial_{\mu} \Psi_{R}}_{\text {Fermion kinetic }} \underbrace{-\bar{\Psi}_{L} \gamma^{\mu}\left(g W_{\mu}^{a} T^{a}+\frac{g^{\prime}}{2} B_{\mu} Y\right) \Psi_{L}}_{\text {Left-handed interaction }} \\
\underbrace{-\bar{\Psi}_{R} \gamma^{\mu} \frac{g^{\prime}}{2} B_{\mu} Y \Psi_{R}}_{\text {Right-handed interaction }} \underbrace{-\frac{1}{4} B_{\mu \nu} B^{\mu \nu}-\frac{1}{4} W_{\mu \nu}^{a} W^{a \mu \nu}}_{\text {Gauge sector }}
\end{array}
$$

Two interaction terms in Eqn. (2.22) describing interactions of left-handed and right-handed fermion fields with the $W_{\mu}^{a}, B_{\mu}$ gauge fields arise from the requirement of $S U(2)_{L} \otimes U(1)_{Y}$ gauge invariance. However, the requirement of gauge invariance forbids the presence of the mass terms of the gauge fields $\frac{1}{2} m^{2} W_{a \mu} W_{a}^{\mu}$ and $\frac{1}{2} m^{2} B_{\mu} B^{\mu}$ in the Lagrangian. Adding the mass term of the fermion fields such as $\frac{1}{2} m^{2}\left(\bar{\psi}_{L} \psi_{R}+\left(\bar{\psi}_{R} \psi_{L}\right)\right.$ would also destroy the gauge invariance of Lagrangian. Therefore, the electroweak Lagrangian (Eqn. (2.22)) describes only massless fermions and massless gauge bosons.

To accommodate the charged vector bosons $\left(W^{ \pm}\right)$, the $W_{\mu}^{+}$and $W_{\mu}^{-}$fields which are responsible for weak-charged current interactions are introduced as complex combinations of the $W_{\mu}^{1}$ and $W_{\mu}^{2}$ gauge fields:

$$
W_{\mu}^{ \pm}=\frac{1}{\sqrt{2}}\left(W_{\mu}^{1} \mp i W_{\mu}^{2}\right)
$$

By defining:

$$
W_{\mu \nu}^{ \pm}=\frac{1}{\sqrt{2}}\left(W_{\mu \nu}^{1} \mp i W_{\mu \nu}^{2}\right),
$$

the gauge sector in Eqn. (2.21) is rewritten as:

$$
\mathcal{L}_{\text {gauge }}=-\frac{1}{4} B_{\mu \nu} B^{\mu \nu}-\frac{1}{4} W_{\mu \nu}^{3} W^{3 \mu \nu}-\frac{1}{2} W_{\mu \nu}^{-} W^{+\mu \nu}
$$

with:

$$
W_{\mu \nu}^{3}=\partial_{\mu} W_{\nu}^{3}-\partial_{\nu} W_{\mu}^{3}-i g\left(W_{\mu}^{-} W_{\nu}^{+}-W_{\nu}^{-} W_{\mu}^{+}\right)
$$




$$
\begin{aligned}
& W_{\mu \nu}^{+}=\left(\partial_{\mu}+i g W_{\mu}^{3}\right) W_{\nu}^{+}-\left(\partial_{\nu}+i g W_{\nu}^{3}\right) W_{\mu}^{+} \\
& W_{\mu \nu}^{-}=\left(\partial_{\mu}-i g W_{\mu}^{3}\right) W_{\nu}^{-}-\left(\partial_{\nu}-i g W_{\nu}^{3}\right) W_{\mu}^{-}
\end{aligned}
$$

The relation described in Eqn. (2.16) implies that the $A_{\mu}$ field associated with the generator $Q$ of $U(1)_{E M}$ must be a combination of the $W_{\mu}^{3}$ and $B_{\mu}$ fields associated with generators $T^{3}$ and $Y$. In a sense, the unification of the electromagnetic and weak interactions is expressed in mixing of $B_{\mu}$ and $W_{\mu}^{3}$ fields to form the $Z_{\mu}$ and $A_{\mu}$ fields associated with the $\mathrm{Z}$ boson and photon:

$$
\begin{aligned}
& Z_{\mu}=\frac{1}{\sqrt{g^{2}+g^{\prime 2}}}\left(g W_{\mu}^{3}-g^{\prime} B_{\mu}\right) \\
& A_{\mu}=\frac{1}{\sqrt{g^{2}+g^{\prime 2}}}\left(g^{\prime} W_{\mu}^{3}+g B_{\mu}\right) .
\end{aligned}
$$

Rather than having a symmetry group with a single coupling to unify electromagnetic and weak interactions, the $S U(2)_{L} \otimes U(1)_{Y}$ theory contains two symmetry groups with independent coupling strength $g$ and $g^{\prime}$. Using the definition of Weinberg or weak mixing angle $\theta_{W}$ :

$$
\tan \theta_{W} \equiv \frac{g^{\prime}}{g}
$$

the $Z_{\mu}$ and $A_{\mu}$ fields are written as:

$$
Z_{\mu}=W_{\mu}^{3} \cos \theta_{W}-B_{\mu} \sin \theta_{W} \quad A_{\mu}=W_{\mu}^{3} \sin \theta_{W}+B_{\mu} \cos \theta_{W}
$$

Inverting the rotation in $\left(B_{\mu}, W_{\mu}^{3}\right)$ space (Eqn. (2.29)) gives the expression for $B_{\mu}$ and $W_{\mu}^{3}$ in term of $Z_{\mu}$ and $A_{\mu}$ :

$$
B_{\mu}=A_{\mu} \cos \theta_{W}-Z_{\mu} \sin \theta_{W} \quad W_{\mu}^{3}=A_{\mu} \sin \theta_{W}+Z_{\mu} \cos \theta_{W}
$$

Substituting Eqn. (2.32) into Eqn. (2.26) and Eqn. (2.19) gives:

$$
\begin{gathered}
B_{\mu \nu}=A_{\mu \nu} \cos \theta_{W}-Z_{\mu \nu} \sin \theta_{W} \\
W_{\mu \nu}^{3}=A_{\mu \nu} \sin \theta_{W}+Z_{\mu \nu} \cos \theta_{W}-i g\left(W_{\mu}^{-} W_{\nu}^{+}-W_{\nu}^{-} W_{\mu}^{+}\right) .
\end{gathered}
$$

Using Eqn. (2.33) and Eqn. (2.34), the gauge sector in Eqn. (2.25) can be rewritten as:

$$
\mathcal{L}_{\text {gauge }}=-\frac{1}{4} Z_{\mu \nu} Z^{\mu \nu}-\frac{1}{4} A_{\mu \nu} A^{\mu \nu}-\frac{1}{2} W_{\mu \nu}^{-} W^{+\mu \nu}
$$


with:

$$
\begin{aligned}
W_{\mu \nu}^{+}= & \left(\partial_{\mu}+i g A_{\mu} \sin \theta_{W}+i g Z_{\mu} \cos \theta_{W}\right) W_{\nu}^{+} \\
& -\left(\partial_{\nu}+i g A_{\nu} \sin \theta_{W}+Z_{\nu} \cos \theta_{W}\right) W_{\mu}^{+} \\
W_{\mu \nu}^{-} & =\left(\partial_{\mu}-i g A_{\mu} \sin \theta_{W}-i g Z_{\mu} \cos \theta_{W}\right) W_{\nu}^{-} \\
- & \left(\partial_{\nu}-i g A_{\nu} \sin \theta_{W}-i g Z_{\nu} \cos \theta_{W}\right) W_{\mu}^{-}
\end{aligned}
$$

The interaction of the electromagnetic field $A_{\mu}(x)$ with vector boson fields $W_{\mu}^{+}(x)$ and $W_{\mu}^{-}(x)$ is shown in the last term of Eqn. (2.35), and there is no term in the Lagrangian (Eqn. (2.35)) describing interaction between electromagnetic field and vector boson fields $Z_{\mu}(x)$. The three vector fields, $W_{\mu}^{+}(x), W^{-}(x)_{\mu}$ and $B_{\mu}(x)$ are identified with the mediators of the weak interaction, $W^{+}, W^{-}$and $Z$ particles. The $W^{ \pm}$bosons are found experimentally to carry electric charge of $\pm e$, and the $Z$ boson is found to be neutral. From the coupling of the $W^{ \pm}$fields to electromagnetic field $\left( \pm g \sin \theta_{W}\right)$ shown in Eqn. (2.35) and Eqn. (2.37), the electromagnetic coupling is identified as:

$$
e=g \sin \theta_{W}=g^{\prime} \cos \theta_{W}
$$

The $W^{ \pm}$are responsible for the charged weak currents, meanwhile, $Z$ is responsible for the neutral weak current.

Explicitly adding the mass terms for the $W_{\mu}^{ \pm}(x)$ and $B_{\mu}(x)$ fields as well as for the fermion fields to the electroweak Lagrangian is prohibited by gauge invariance. However, experimental results have confirmed that all gauge bosons of weak interactions are massive. To introduce the mass of $W^{ \pm}$and $\mathrm{Z}$ without explicitly breaking the local gauge invariance, a mechanism of spontaneous symmetry breaking has been developed to reveal the mass for those bosons corresponding to the broken generators of the symmetry group. This intriguing development will be described in Sec. 2.1.3 on the Higgs Mechanism.

\section{Strong interaction : $\mathrm{SU}(3)_{\mathrm{C}}$}

Quantum Chromodynamics (QCD) is a theory describing the strong interaction between particles carrying color charges. In the Standard Model, each flavor of quark has three color states and QCD is based on the $S U(3)$ gauge group of phase transformations on the quark color fields. Leptons do not carry color charge, therefore, they do not participate in the strong interaction and are singlets under $S U(3)$ transformation.

Consider the Lagrangian for a free quark:

$$
\mathcal{L}=\bar{\psi}_{q}\left(i \gamma^{\mu} \partial_{\mu}-m\right) \psi_{q}
$$


Under the $S U(3)_{C}$ gauge group, the quark fields of each flavor transforms as a color triplet:

$$
\psi_{q} \rightarrow \psi_{q}^{\prime}=e^{i \alpha_{a}(x) L_{a}} \psi_{q}
$$

where $\alpha_{a}(\mathrm{a}=1, \ldots, 8)$ are real parameters and $L_{a}$ are generators of the $S U(3)$ gauge group. In the fundamental representation, $L_{a}$ are conventionally written in terms of $3 \times 3$ traceless Gell-Mann matrices:

$$
L_{a}=\frac{\lambda_{a}}{2}
$$

with

$$
\begin{array}{rlrl}
\lambda_{1} & =\left(\begin{array}{ccc}
0 & 1 & 0 \\
1 & 0 & 0 \\
0 & 0 & 0
\end{array}\right) & \lambda_{2} & =\left(\begin{array}{ccc}
0 & -i & 0 \\
i & 0 & 0 \\
0 & 0 & 0
\end{array}\right) \\
\lambda_{3}=\left(\begin{array}{ccc}
1 & 0 & 0 \\
0 & -1 & 0 \\
0 & 0 & 0
\end{array}\right) & \lambda_{4}=\left(\begin{array}{ccc}
0 & 0 & 1 \\
0 & 0 & 0 \\
1 & 0 & 0
\end{array}\right) \\
\lambda_{5}=\left(\begin{array}{ccc}
0 & 0 & -i \\
0 & 0 & 0 \\
i & 0 & 0
\end{array}\right) & \lambda_{6}=\left(\begin{array}{ccc}
0 & 0 & 0 \\
0 & 0 & 1 \\
0 & 1 & 0
\end{array}\right) \\
\lambda_{7}=\left(\begin{array}{ccc}
0 & 1 & 0 \\
1 & 0 & -i \\
0 & i & 0
\end{array}\right) & \lambda_{8}=\frac{1}{\sqrt{3}}\left(\begin{array}{ccc}
1 & 0 & 0 \\
0 & 1 & 0 \\
0 & 0 & -2
\end{array}\right)
\end{array}
$$

Similar to the $U(1)_{E M}$ theory of electromagnetic interaction and the $S U(2)_{L} \otimes U(1)_{Y}$ theory of electroweak interaction, postulating the invariance of the Lagrangian (Eqn. (2.39)) under the $S U(3)$ local gauge transformation (Eqn. (2.40)) gives rise to eight vector gauge fields $G_{\mu}^{a}$ and forces us to replace the partial derivative with the covariant derivative. Just as for the photon, local gauge invariance also requires gluons, excitations of the gauge fields $G_{\mu}^{a}$ mediating the strong force, to be massless. Therefore, the QCD Lagrangian is given as:

$$
\mathcal{L}=\bar{\psi}_{q}\left(i \gamma^{\mu} D_{\mu}-m\right) \psi_{q}-\frac{1}{4} G_{\mu \nu}^{a} G_{a}^{\mu \nu}
$$

where $D_{\mu}$ is covariant derivative and $G_{\mu \nu}^{a}$ are gauge field strength tensors:

$$
\begin{gathered}
D_{\mu}=\partial_{\mu}+i g_{s} G_{\mu}^{a} L_{a} \\
G_{\mu \nu}^{a}=\partial_{\mu} G_{\nu}^{a}-\partial_{\nu} G_{\mu}^{a}-g_{s} f_{a b c} G_{\mu}^{b} G_{\nu}^{c} .
\end{gathered}
$$

In above equations, the parameter $g_{s}$ is the strong coupling constant and $f_{a b c}$ is the structure constant of the $S U(3)$ group $\left[L^{a}, L^{b}\right]=i f_{a b c} L^{c}$. Under the $S U(3)_{C}$ gauge group, the gluons 
fields transforms as:

$$
G_{\mu}^{a} \rightarrow G_{\mu}^{a}-\frac{1}{g_{s}} \partial_{\mu} \alpha^{a}-f_{a b c} \alpha^{b} G_{\mu}^{c}
$$

Interactions in QCD are revealed by rearranging the terms in Lagrangian (2.43):

$$
\begin{gathered}
\mathcal{L}_{Q C D}=\underbrace{\bar{\psi}_{q}\left(i \gamma^{\mu} \partial_{\mu}+m\right) \psi_{q}}_{\text {Quark kinetic and mass term }}-\underbrace{\frac{1}{4}\left(\partial_{\mu} G_{\nu}^{a}-\partial_{\nu} G_{\mu}^{a}\right)\left(\partial^{\mu} G_{a}^{\nu}-\partial^{\nu} G_{a}^{\mu}\right)}_{\text {Gauge field kinetic }} \\
+\underbrace{g_{s} \bar{\psi}_{q} \gamma^{\mu} G_{\mu}^{a} L^{a} \psi_{q}}_{\text {Quark-gluons interaction }}+\underbrace{g_{s} f_{a b c}\left(\partial_{\mu} G_{a}^{\nu}\right) G_{b}^{\mu} G_{c}^{\nu}+g_{s}^{2}\left(f_{a b c} G_{b}^{\mu} G_{c}^{\nu}\right)\left(f_{a d e} G_{d}^{\mu} G_{e}^{\nu}\right)}_{\text {Gluon self-interaction }}
\end{gathered}
$$

Arising from imposing the $S U(3)$ local gauge invariance on the Lagrangian of free quarks, the quark-gluon interaction term in the QCD Lagrangian describes the interaction of quarks and gluon fields. The 3-point and 4-point self interactions of gluons appear in the QCD Lagrangian as a consequence of gauge invariance requirement and the non-Abelian character of the $S U(3)$ gauge group. The gluon self interactions reflect the fact that gluons themselves carry color charge.

At very short distance $(<0.1 \mathrm{fm})$ or very high energy $(>10 \mathrm{GeV})$, the effective strong coupling constant becomes so small that quarks and gluons can be considered as approximately free and their interactions can be treated by perturbation theory. This remarkable property of strong interaction is due to the non-Abelian nature of QCD and known as asymptotic freedom. At long distances or small values of energy, the effective strong interaction coupling constant becomes large and the theory is no longer perturbative. On a length scale greater than about $1 \mathrm{fm}$, the quark and gluon fields are experimentally known to be confining and colored particles such as quarks and gluons can never be isolated. The peculiar property of strong interaction that does not allow isolated quarks to exist, and binding quarks together forming colorless hadrons is called confinement.

\subsubsection{The Higgs Mechanism}

It has been well experimentally established that the weak interaction is mediated by massive $W^{+}, W^{-}$and $Z$ bosons. To incorporate the mass of the $W^{+}, W^{-}$and $Z$ bosons into the Standard Model where the gauge theory requires all gauge bosons to be massless, the Higgs mechanism has been developed to circumvent this constraint on the mass. The Higgs mechanism begins with applying a local gauge invariant theory with massless gauge bosons to hypothetical scalar fields and ends with a Standard Model spectrum having massive gauge bosons, after undergoing a spontaneous symmetry breaking.

Consider a Higgs doublet of complex scalar fields $\phi=\left(\begin{array}{c}\phi^{+} \\ \phi^{0}\end{array}\right)$, where the charged and 
neutral components of the doublet are constructed from four real scalar fields:

$$
\phi^{+}=\frac{1}{\sqrt{2}}\left(\phi_{1}+i \phi_{2}\right) \quad \phi^{0}=\frac{1}{\sqrt{2}}\left(\phi_{3}+i \phi_{4}\right)
$$

The week isospins $\left(T, T^{3}\right)$ and hypercharge (Y) quantum numbers of the Higgs doublet are chosen as in Table 2.4 .

\begin{tabular}{||c|c|c|c||}
\hline \hline Field & Isospin T & Isospin $\mathbf{T}^{\mathbf{3}}$ & Hypercharge Y \\
\hline$\phi^{+}$ & $\frac{1}{2}$ & $\frac{1}{2}$ & 1 \\
\hline$\phi^{0}$ & $\frac{1}{2}$ & $-\frac{1}{2}$ & 1 \\
\hline \hline
\end{tabular}

Table 2.4: Properties of Higgs doublet

In the $S U(2)_{L} \otimes U(1)_{Y}$ gauge theory where the massless vector gauge fields $W_{\mu}^{a}$ and $B_{\mu}$ have been introduced, the local gauge invariant Lagrangian describing the Higgs doublet is given as:

$$
\mathcal{L}_{\text {Higgs }}=\left|\left(\partial_{\mu}+i g W_{\mu}^{a} T^{a}+i \frac{g^{\prime}}{2} B_{\mu} Y\right) \phi\right|^{2}-V(\phi),
$$

where $V(\phi)$ is the Higgs potential:

$$
V(\phi)=\mu^{2} \phi^{\dagger} \phi+\lambda\left(\phi^{\dagger} \phi\right)^{2}
$$

The parameters in Eqn. (2.50) are constrained by $\mu^{2}<0$ and $\lambda>0$ so that the Higgs potential has a minimum different than zero and is bounded from below (Fig. 2.1). Then the potential defined by Eqn. (2.50) reaches its minimum at a finite value of $|\phi|$ where:

$$
|\phi|^{2} \equiv \phi^{\dagger} \phi \equiv \frac{1}{2}\left(\phi_{1}^{2}+\phi_{2}^{2}+\phi_{3}^{2}+\phi_{4}^{2}\right)=-\frac{\mu^{2}}{2 \lambda}
$$

The vacuum state of the system is degenerated in four-dimentional space of scalar fields $\left(\phi_{1}, \phi_{2}, \phi_{3}, \phi_{4}\right)$, and the manifold of points at which $V(\phi)$ is minimized is invariant under the transformation of $S U(2)_{L} \otimes U(1)_{Y}$ symmetry. The fluctuations around the minimum energy are calculated by perturbation theory based on expanding $\phi(x)$ about a particular vacuum state which has been chosen as:

$$
\phi_{1}=\phi_{2}=\phi_{4}=0, \quad \phi_{3}^{2}=-\frac{\mu^{2}}{\lambda}=v^{2}
$$

Parameterizing the fluctuations from the vacuum state chosen as Eqn. (2.52) in terms of 


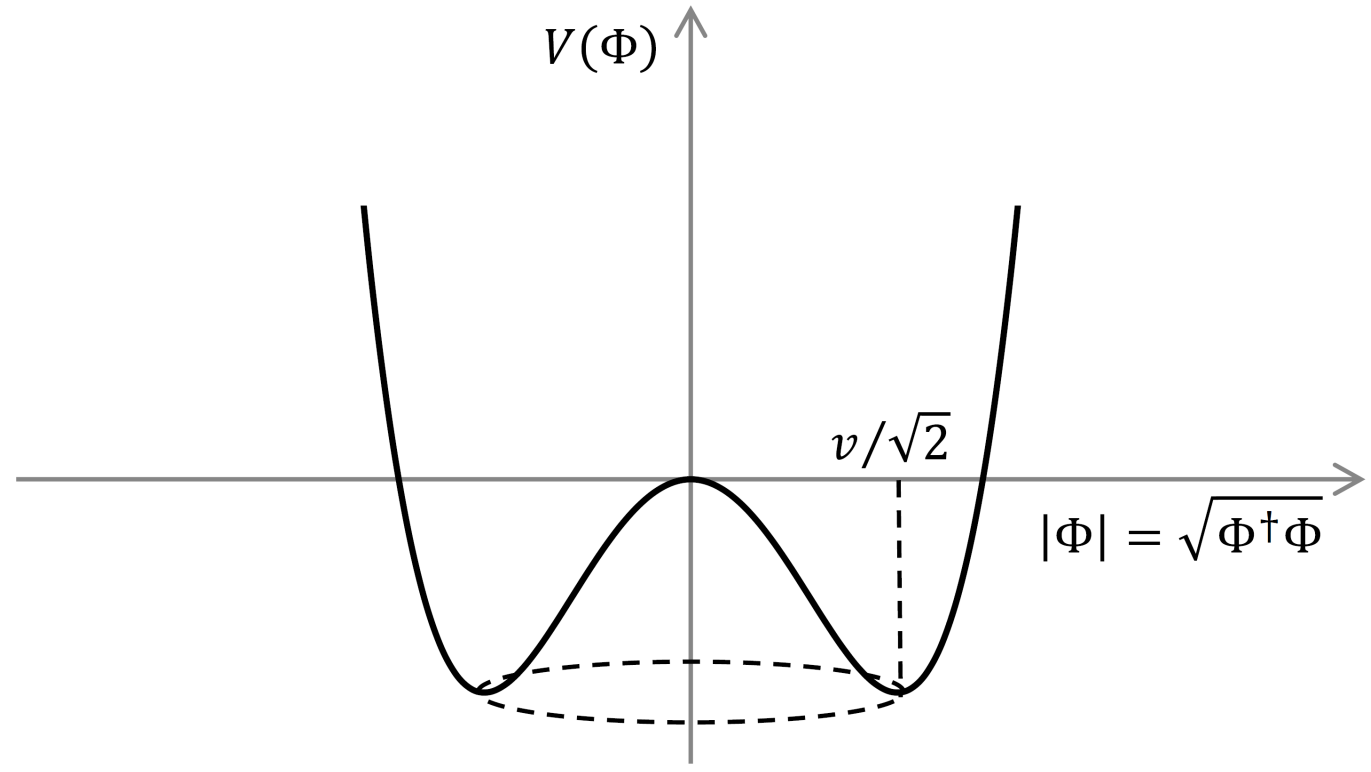

Figure 2.1: The Higgs potential

four real fields $\boldsymbol{\xi}\left(\xi_{1}(x), \xi_{2}(x), \xi_{3}(x)\right)$ and $h(x)$ with corresponding vacuum expectation value (VEV):

$$
\left\langle\xi_{1}(x)\right\rangle=\left\langle\xi_{2}(x)\right\rangle=\left\langle\xi_{3}(x)\right\rangle=0, \quad\langle h(x)\rangle=0,
$$

the exited states of the Higgs doublet are obtained in the form:

$$
\phi(x)=\frac{1}{\sqrt{2}}\left(\begin{array}{c}
\xi_{2}(x)+i \xi_{1}(x) \\
v+h(x)-i \xi_{3}(x)
\end{array}\right) \approx e^{i \boldsymbol{\tau} \cdot \xi(x) / v}\left(\begin{array}{c}
0 \\
\frac{v+h(x)}{\sqrt{2}}
\end{array}\right),
$$

with choice of the unitary gauge $U(\xi)=e^{-i \boldsymbol{\tau} \cdot \xi(x) / v}$. Due to the gauge invariance, the exited states (Eqn. (2.54)) in the unitary gauge can be written as:

$$
\phi(x)=\left(\begin{array}{c}
0 \\
\frac{v+h(x)}{\sqrt{2}}
\end{array}\right)
$$

Adding a constant term $\left(-\mu^{2} v^{2} / 4\right)$ to Eqn. (2.50) and substituting Eqn. (2.55) in, the Higgs potential is expressed in terms of the VEV and the field $h(x)$ :

$$
\begin{array}{r}
V(\phi)=\lambda\left(\phi^{\dagger} \phi-\frac{v^{2}}{2}\right)^{2} \\
V(\phi)=V(h)=-\mu^{2} h^{2}-\frac{\mu^{2}}{v} h^{3}-\frac{\mu^{2}}{4 v^{2}} h^{4}
\end{array}
$$

Substituting Eqn. (2.55) and Eqn. (2.56) in Eqn. (2.49), and using the relations described 
in Eqn. (2.23) and Eqn. (2.31), we find:

$$
\mathcal{L}_{\text {Higgs }}=\frac{1}{2} \partial_{\mu} h \partial^{\mu} h-\frac{g^{2}}{4} W_{\mu}^{-} W^{+\mu}(v+h)^{2}+\frac{1}{8}\left(g^{2}+g^{\prime 2}\right) B_{\mu} B^{\mu}(v+h)^{2}-V(h)
$$

There are infinite number of possible Higgs doublet vacuum states which have the VEV of $v / \sqrt{2}$. Choosing a particular state as in Eqn. (2.52) is equivalent to spontaneously breaking $S U(2)_{L} \otimes U(1)_{Y}$ symmetry, and the Lagrangian in Eqn. (2.57) obtained from expanding $\phi(x)$ about this particular state is no longer invariant under the $S U(2)_{L} \otimes U(1)_{Y}$ gauge transformation. However, the option of giving the VEV to the neutral component of the Higgs doublet ensures the conservation of electric charge and the $U(1)_{E M}$ symmetry remains unbroken after the expansion.

The physics content of the full Lagragian after the spontaneous symmetry breaking is revealed by adding Eqn. (2.35) to Eqn. (2.57) and rearranging the terms as:

$$
\begin{aligned}
\mathcal{L}= & \mathcal{L}_{\text {Higgs }}+\mathcal{L}_{\text {gauge }} \\
\mathcal{L}= & \frac{1}{2} \partial_{\mu} h \partial^{\mu} h+\mu^{2} h^{2}+\frac{\mu^{2}}{v} h^{3}+\frac{\mu^{2}}{4 v^{2}} h^{4} \\
& -\frac{1}{2} W_{\mu \nu}^{-} W^{+\mu \nu}+\frac{1}{4} v^{2} g^{2} W_{\mu}^{-} W^{+\mu}-\left(\frac{h^{2}}{4}+\frac{v h}{2}\right) g^{2} W_{\mu}^{-} W^{+\mu} \\
& -\frac{1}{4} Z_{\mu \nu} Z^{\mu \nu}-\frac{1}{8} v^{2}\left(g^{2}+g^{\prime 2}\right) B_{\mu} B^{\mu}-\left(\frac{h^{2}}{8}+\frac{v h}{4}\right)\left(g^{2}+g^{\prime 2}\right) Z_{\mu} Z^{\mu} \\
& -\frac{1}{4} A_{\mu \nu} A^{\mu \nu}
\end{aligned}
$$

The resulting Eqn. (2.58) describes the Lagrangian for a free massive neutral scalar boson field $h(x)$, a free massive neutral vector boson field $Z_{\mu}(x)$, a pair of massive charged vector boson fields $W_{\mu}^{ \pm}(x)$, and a massless photon field $A_{\mu}(x)$. The Higgs doublet $\phi(x)$ and its vacuum state have been constructed with the requirement that the photon be massless, therefore, the result $M_{A}^{2}=0$ is a consistency check on the calculation in the Higgs mechanism. From the expected mass term for a charge boson $\left(M_{W}^{2}=W_{\mu}^{-} W^{+\mu}\right)$, and that for a neutral boson $\left(M_{Z}^{2}=Z_{\mu} Z^{\mu}\right)$, the masses of $W$ and $Z$ bosons are identified as:

$$
\begin{aligned}
M_{W} & =\frac{1}{2} v g \\
M_{Z} & =\frac{1}{2} v \sqrt{\left(g^{2}+g^{\prime 2}\right)}
\end{aligned}
$$

Subsequently, the $W^{ \pm}$and $Z$ bosons were discovered with the masses [17]:

$$
\begin{aligned}
M_{W} & =80.399 \pm 0.023 \mathrm{GeV} \\
M_{Z} & =91.1876 \pm 0.0021 \mathrm{GeV}
\end{aligned}
$$

Using the notation introduced in Eqn. (2.30) and predicted $W$ and $Z$ masses in Eqn. (2.59), the relation between $W$ and $Z$ masses is expressed in terms of the weak mixing angle $\theta_{W}$ 
as:

$$
\frac{M_{W}}{M_{Z}}=\cos \theta_{W}=0.8819 \pm 0.0006
$$

Among the four fields parameterizing the Higgs doublet in Eqn. (2.54), three fields $\left(\xi_{1}(x), \xi_{2}(x), \xi_{3}(x)\right)$ have been gauged away, and there remains only the scalar Higgs field $h(x)$ which can provide a handle to verify the theory. From Eqn. (2.59), the vacuum expectation value of the Higgs field is estimated as:

$$
\frac{v}{\sqrt{2}}=\frac{\sqrt{2} M_{W}}{g}=\frac{\sqrt{2} M_{W} \sin \theta_{W}}{e} \approx 174 \mathrm{GeV}
$$

There are three-point and four-point interaction terms of the Higgs field $h(x)$ and the weak gauge boson fields shown in Eqn. (2.58). Using the expressions of the weak boson masses in Eqn. (2.59), the three-point coupling strength of the Higgs field to the $W^{ \pm}$and $Z$ boson fields are found to be proportional to the mass of the gauge bosons:

$$
g M_{W}, \quad \frac{g M_{Z}}{\cos \theta_{W}} .
$$

Imposing $S U(2)_{L} \otimes U(1)_{Y}$ gauge invariance on the Lagrangian, followed by the spontaneous symmetry breaking $S U(2)_{L} \otimes U(1)_{Y} \rightarrow U(1)_{E M}$, generates the massive vector $W \pm$ and $Z$ gauge boson fields, the massless electromagnetic field and gives a rise to the Higgs scalar field $h(x)$. The excitation of the neutral scalar $h(x)$ field, the Higgs boson, is an electrically neutral particle with mass determined from parameters of the Higgs potential:

$$
M_{H}=\sqrt{2 v^{2} \lambda}
$$

The parameter $v$ is experimentally fixed by Eqn. (2.61), but $\lambda$ is a free parameter in the Standard Model. Therefore, the mass of the Higgs boson is not predicted by theory. However, the existence of the Higgs boson is an essential part of the Standard Model. Theoretical studies to put constraints on the Higgs boson mass as well as experimental searches for the evidence of this particle have been pursued for half a century. The experimental confirmation of the existence of the SM Higgs boson has been eagerly awaited.

\subsubsection{Yukawa Interaction and Fermion Mass}

Combining the gauge theories describing electroweak and strong interactions together, the general symmetry group of the SM is formed as a product of three disconnected sets of gauge transformations with different coupling strengths, the $S U(3)_{C}$ group with strength 
$g_{s}$, the $S U(2)_{L}$ group with strength $g$ and $U(1)_{Y}$ with strength $g^{\prime}$ :

$$
G_{S M}=\mathrm{SU}(3)_{C} \otimes \mathrm{SU}(2)_{L} \otimes \mathrm{U}(1)_{Y}
$$

The quarks and leptons of each generation in the SM are excitations of fermion fields which are organized in five representations of the gauge symmetry group $G_{S M}$ :

$$
Q_{L i}^{I}(3,2)_{+1 / 3}, \quad U_{R i}^{I}(3,1)_{+4 / 3}, \quad D_{R i}^{I}(3,1)_{-2 / 3}, \quad L_{L i}^{I}(1,2)_{-1}, \quad E_{R i}^{I}(1,1)_{-2}
$$

In the fermion representations (2.65), the super-index $I$ denotes gauge interaction eigenstates, the sub-index $i=1,2,3$ is the generation index, the first and second numbers in the parenthesis indicate the $\mathrm{SU}(3)$ and $\mathrm{SU}(2)$ representations respectively, and subindex number represents $\mathrm{U}(1)_{Y}$ charge. $Q_{L i}^{I}(3,2)_{+1 / 6}$ and $L_{L i}^{I}(1,2)_{-1 / 2}$ are left-handed quark and lepton fields, respectively. $U_{R i}^{I}(3,1)_{+2 / 3}$ and $D_{R i}^{I}(3,1)_{-1 / 3}$ are right-handed up-type and down-type quark fields. $E_{R i}^{I}(1,1)_{-1}$ represents right-handed charged lepton fields.

The Higgs doublet of complex scalar fields introduced in the Higgs mechanism is a singlet of $S U(3)_{\mathrm{C}}$, doublet of $S U(2)_{\mathrm{L}}$ and carries hypercharge $Y=+1 / 2$. It can be expressed as a scalar representation of the gauge symmetry group:

$$
\phi(1,2)_{+1} .
$$

Explicitly adding the lepton or quark mass terms $(-m \psi \bar{\psi})$ to the Lagrangian of the fermion system in Eqn. (2.22) violates the gauge theory. To retain the gauge invariance, the fermion mass needs to be introduced into the SM model through the Yukawa interaction between the Higgs field and the fermions fields. The contribution to the Lagrangian of the coupling for three fermion families to the Higgs field is taken to be:

$$
\begin{aligned}
& \mathcal{L}_{\text {Yukawa }}=\mathcal{L}_{\text {Yukawa }}^{\text {lepton }}+\mathcal{L}_{\text {Yukawa }}^{\text {quark }} \\
& \mathcal{L}_{\text {Yukawa }}^{\text {leptons }}=-Y_{i j}^{e} \overline{L_{L i}^{I}} \phi E_{R j}^{I}-Y_{i j}^{e \star} \overline{E_{R j}^{I}} \bar{\phi} L_{L i}^{I} \\
& \mathcal{L}_{\text {Yukawa }}^{\text {quarks }}=-Y_{i j}^{d} \overline{Q_{L i}^{I}} \phi D_{R j}^{I}-Y_{i j}^{u} \overline{Q_{L i}^{I}} \tilde{\phi} U_{R j}^{I}-Y_{i j}^{d \star} \overline{D_{R j}^{I}} \overline{\tilde{\phi}} Q_{L i}^{I}-Y_{i j}^{u \star} \overline{U_{R j}^{I}} \bar{\phi} Q_{L i}^{I}
\end{aligned}
$$

where $Y_{i j}^{e}, Y_{i j}^{d}$ and $Y_{i j}^{u}$ are arbitrary complex matrixes, and the conjugate of the Higgs field $\tilde{\phi}$ is constructed as :

$$
\tilde{\phi}(1,2)_{-1}=-i \tau_{2} \phi^{\star}=\left(\begin{array}{c}
-\overline{\phi^{0}} \\
\phi^{-}
\end{array}\right)
$$

Gathering all ingredients together, we have the Standard Model Lagrangian $\mathcal{L}_{S M}$, which is consistent with the gauge symmetry in Eqn. (2.64) and particle content shown in Eqns. (2.65) and (2.66), written as:

$$
\mathcal{L}_{\mathrm{SM}}=\mathcal{L}_{\text {kinetic }}+\mathcal{L}_{\text {Higgs }},+\mathcal{L}_{\text {Yukawa }}
$$


where $\mathcal{L}_{\text {Yukawa }}(2.67)$ describes the Yukawa coupling and $\mathcal{L}_{\text {Higgs }}$ represents the scalar self interaction:

$$
\mathcal{L}_{\text {Higgs }}=-\mu^{2} \phi^{\dagger} \phi-\lambda\left(\phi^{\dagger} \phi\right)^{2} .
$$

The $\mathcal{L}_{\text {kinetic }}$ includes kinematic terms of all the fields:

$$
\begin{aligned}
\mathcal{L}_{\text {kinetic }}= & \mathcal{L}_{\text {kinetic }}^{\text {lepton }}+\mathcal{L}_{\text {kinetic }}^{\text {quark }}+\mathcal{L}_{\text {kinetic }}^{\text {gauge }}+\mathcal{L}_{\text {kinetic }}^{\text {Higgs }} \\
\mathcal{L}_{\text {kinetic }}^{\text {lepton }}= & \overline{L_{L i}^{I}} \gamma_{\mu}\left(i \partial^{\mu}-g W^{a \mu} T^{a}-\frac{g^{\prime}}{2} B^{\mu} Y\right) L_{L i}^{I}+\overline{E_{R i}^{I}} \gamma_{\mu}\left(i \partial^{\mu}-\frac{g^{\prime}}{2} B^{\mu} Y\right) E_{R i}^{I} \\
\mathcal{L}_{\text {kinetic }}^{\text {quark }}= & \overline{Q_{L i}^{I}} \gamma_{\mu}\left(i \partial^{\mu}-g_{s} G^{b \mu} L^{b}-g W^{a \mu} T^{a}-\frac{g^{\prime}}{2} B^{\mu} Y\right) Q_{L i}^{I} \\
& +\overline{U_{R i}^{I}} \gamma_{\mu}\left(i \partial^{\mu}-g_{s} G^{b \mu} L^{b}-\frac{g^{\prime}}{2} B^{\mu} Y\right) U_{R i}^{I} \\
& +\overline{D_{R i}^{I}} \gamma_{\mu}\left(i \partial^{\mu}-g_{s} G^{b \mu} L^{b}-\frac{g^{\prime}}{2} B^{\mu} Y\right) D_{R i}^{I} \\
\mathcal{L}_{\text {kinetic }}^{\text {Higgs }}= & \left|\left(\partial_{\mu}+i g W_{\mu}^{a} T^{a}+i \frac{g^{\prime}}{2} B_{\mu} Y\right) \phi\right|^{2} \\
\mathcal{L}_{\text {kinetic }}^{\text {Gauge }=} & -\frac{1}{4} B_{\mu \nu} B^{\mu \nu}-\frac{1}{4} W_{\mu \nu}^{a} W^{a \mu \nu}-\frac{1}{4} G_{\mu \nu}^{b} G^{b \mu \nu}
\end{aligned}
$$

Expanding the SM Lagrangian $\mathcal{L}_{S M}$ around the vacuum states of the Higgs field is equivalent to substituting $\phi(1,2)_{+1}$ and $\tilde{\phi}(1,2)_{-1}$ in Eqn. (2.69) with:

$$
\phi=\left(\begin{array}{c}
-\phi^{+} \\
\phi^{0}
\end{array}\right) \longrightarrow\left(\begin{array}{c}
0 \\
\frac{v+h(x)}{\sqrt{2}}
\end{array}\right), \quad \tilde{\phi}=\left(\begin{array}{c}
-\overline{\phi^{0}} \\
\phi^{-}
\end{array}\right) \longrightarrow\left(\begin{array}{c}
\frac{v+h(x)}{\sqrt{2}} \\
0
\end{array}\right)
$$

Under spontaneous symmetry breaking, the Standard Model symmetry group $G_{S M}$ breaks down as:

$$
\mathrm{SU}(3)_{C} \otimes \mathrm{SU}(2)_{L} \otimes \mathrm{U}(1)_{Y} \rightarrow \mathrm{SU}(3)_{C} \otimes \mathrm{U}(1)_{E M}
$$

The gluons and the photons associated with generators of the unbroken parts, $S U(3)_{C}$ and $U(1)_{E M}$, remain massless, while $W^{ \pm}$and $Z$ weak bosons acquire a masses as shown in Eqn. (2.59).

Decomposing the $S U(2)_{L}$ lepton and quark doublets into their components as:

$$
L_{L i}^{I}(1,2)_{-1}=\left(\begin{array}{c}
\nu_{e L}^{I} \\
e_{L}^{I}
\end{array}\right), \quad\left(\begin{array}{c}
\nu_{\mu L}^{I} \\
\mu_{L}^{I}
\end{array}\right), \quad\left(\begin{array}{c}
\nu_{\tau L}^{I} \\
\tau_{L}^{I}
\end{array}\right) ; \quad Q_{L i}^{I}=\left(\begin{array}{c}
U_{L i}^{I} \\
D_{L i}^{I}
\end{array}\right)
$$

and applying the substitution Eqn. (2.72) to Eqn. (2.67), we obtain the Yukawa interaction terms of the SM Lagrangian expressed in terms of the vacuum expectation value and of the neutral scalar Higgs field $h(x)$ as:

$$
\mathcal{L}_{\text {Yukawa }}^{\text {leptons }}=-\frac{v+h}{\sqrt{2}}\left(\overline{e_{L}^{I}}, \overline{\mu_{L}^{I}}, \overline{\tau_{L}^{I}}\right) \mathrm{Y}^{\mathrm{e}}\left(e_{R}^{I}, \mu_{R}^{I}, \tau_{R}^{I}\right)^{T}+\text { hermitian conjugate }
$$




$$
\begin{aligned}
\mathcal{L}_{\text {Yukawa }}^{\text {quarks }}= & -\frac{v+h}{\sqrt{2}}\left(\overline{D_{L 1}^{I}}, \overline{D_{L 2}^{I}}, \overline{D_{L 3}^{I}}\right) \mathrm{Y}^{\mathrm{d}}\left(D_{R 1}^{I}, D_{R 2}^{I}, D_{R 3}^{I}\right)^{T} \\
& -\frac{v+h}{\sqrt{2}}\left(\overline{U_{L 1}^{I}}, \overline{U_{L 2}^{I}}, \overline{U_{L 3}^{I}}\right) \mathrm{Y}^{\mathrm{u}}\left(U_{R 1}^{I}, U_{R 2}^{I}, U_{R 3}^{I}\right)^{T}+\text { hermitian conjugat( }(2.76)
\end{aligned}
$$

Upon spontaneous symmetry breaking, the Yukawa interactions give rise to the mass terms of leptons and quarks:

$$
\begin{aligned}
\mathcal{L}_{\text {Mass }}^{\text {leptons }}= & -\frac{v}{\sqrt{2}}\left(\overline{e_{L}^{I}}, \overline{\mu_{L}^{I}}, \overline{\tau_{L}^{I}}\right) \mathrm{Y}^{\mathrm{e}}\left(e_{R}^{I}, \mu_{R}^{I}, \tau_{R}^{I}\right)^{T}+\text { hermitian conjugate } \\
\mathcal{L}_{\text {Yukawa }}^{\text {quarks }}= & -\frac{v}{\sqrt{2}}\left(\overline{D_{L 1}^{I}}, \overline{D_{L 2}^{I}}, \overline{D_{L 3}^{I}}\right) \mathrm{Y}^{\mathrm{d}}\left(D_{R j}^{I}, D_{R j}^{I}, D_{R j}^{I}\right)^{T} \\
& -\frac{v}{\sqrt{2}}\left(\overline{U_{L 1}^{I}}, \overline{U_{L 2}^{I}}, \overline{U_{L 3}^{I}}\right) \mathrm{Y}^{\mathrm{u}}\left(U_{R 1}^{I}, U_{R 2}^{I}, U_{R 3}^{I}\right)^{T}+\text { hermitian conjugate }
\end{aligned}
$$

Each matrix of $Y^{e}, Y^{u}, Y^{d}$ can be diagonalized with a set of two chosen $3 \times 3$ unitary matrixes:

$$
Y_{\text {diag }}^{e}=\mathrm{V}_{\mathrm{L}}^{\mathrm{e}} \mathrm{Y}^{\mathrm{e}} \mathrm{V}_{\mathrm{R}}^{\mathrm{e} \dagger}, \quad Y_{\text {diag }}^{u}=\mathrm{V}_{\mathrm{L}}^{\mathrm{u}} \mathrm{Y}^{\mathrm{u}} \mathrm{V}_{\mathrm{R}}^{\mathrm{u} \dagger}, \quad Y_{\text {diag }}^{d}=\mathrm{V}_{\mathrm{L}}^{\mathrm{d}} \mathrm{Y}^{\mathrm{d}} \mathrm{V}_{\mathrm{R}}^{\mathrm{d} \dagger}
$$

and the mass eigenstates of quarks and leptons are defined as:

$$
\begin{aligned}
U_{L i} & =\left(V_{L}^{u}\right)_{i j} U_{L j}^{I} \\
U_{R i} & =\left(V_{R}^{u}\right)_{i j} U_{R j}^{I} \\
D_{L i} & =\left(V_{L}^{d}\right)_{i j} D_{L j}^{I} \\
D_{R i} & =\left(V_{R}^{d}\right)_{i j} D_{R j}^{I} \\
\left(e_{L}, \mu_{L}, \tau_{L}\right)^{T} & =V_{L}^{e}\left(e_{L}^{I}, \mu_{L}^{I}, \tau_{L}^{I}\right)^{T} \\
\left(e_{R}, \mu_{R}, \tau_{R}\right)^{T} & =V_{R}^{e}\left(e_{R}^{I}, \mu_{R}^{I}, \tau_{R}^{I}\right)^{T}
\end{aligned}
$$

Then, from $(2.77),(2.79)$ and (2.80), the masses of the leptons are given by:

$$
m_{e}=\frac{f_{e} v}{\sqrt{2}}, \quad m_{\mu}=\frac{f_{\mu} v}{\sqrt{2}}, \quad m_{\tau}=\frac{f_{\tau} v}{\sqrt{2}},
$$

where the diagonal elements of $Y_{\text {diag }}^{e}, f_{e}, f_{\mu}$, and $f_{\tau}$, are dimensionless Yukawa coupling constants.

Similarly, the masses of the six different flavor quarks are obtained from Eqns. (2.78), (2.79) and (2.80):

$$
\begin{array}{lll}
m_{u}=\frac{f_{u} v}{\sqrt{2}}, & m_{c}=\frac{f_{c} v}{\sqrt{2}}, & m_{t}=\frac{f_{t} v}{\sqrt{2}} \\
m_{d}=\frac{f_{d} v}{\sqrt{2}}, & m_{s}=\frac{f_{s} v}{\sqrt{2}}, & m_{b}=\frac{f_{b} v}{\sqrt{2}},
\end{array}
$$


It can be seen from Eqn. (2.75) and Eqn. (2.76) that introducing masses for charged leptons and quarks through the Yukawa interaction also gives a rise to the interaction between those fermions and the Higgs boson with coupling strength being proportional to the fermion mass:

$$
f=\frac{\sqrt{2} m_{f}}{v}
$$

Couplings of the Higgs boson to itself as well as to fermions and gauge bosons are characterized by the Feynman diagrams in Fig. 2.2.

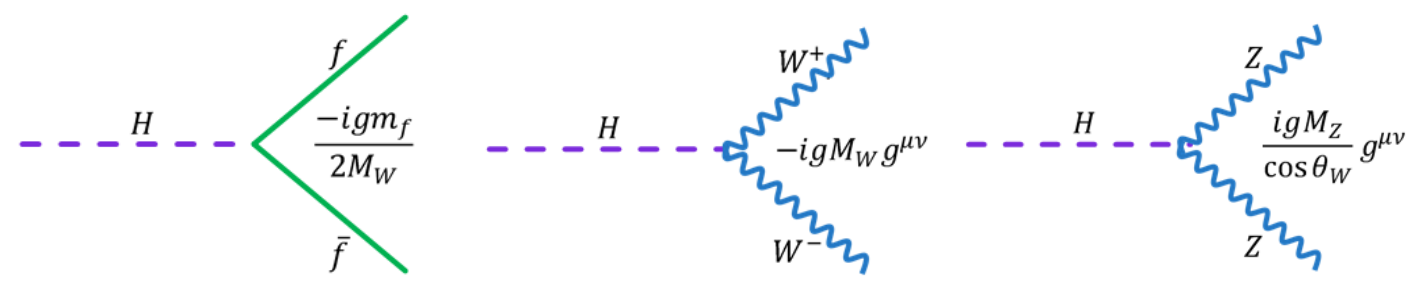

(a)

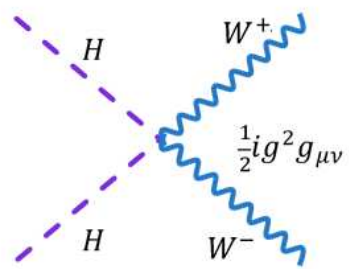

(d) (b)

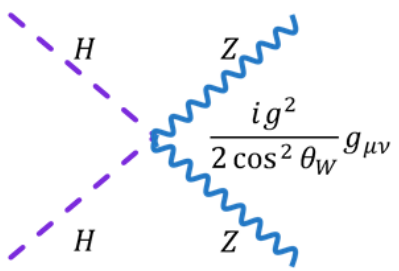

(e) (c)

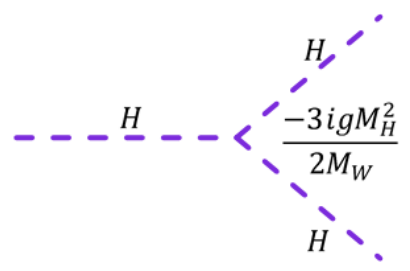

(f)

Figure 2.2: Feynman rules for the SM Higgs Boson

\subsection{The Standard Model Higgs Physics}

\subsubsection{Theoretical Constraints on the Higgs Boson Mass}

The Higgs mechanism in the Standard Model requires the presence of the Higgs boson as a direct physical manifestation of the origin of the mass. The Higgs boson appears to be an electrically neutral particle which has mass depending on two parameters, $M_{H}=\sqrt{2 v^{2} \lambda}$. The parameter $v$ related to vacuum expectation value of the Higgs field is experimentally determined by the mass of the weak gauge bosons. So far, there has been no way to calculate the Higgs boson self-coupling constant $\lambda$, which characterizes the scalar potential, without having experimental knowledge about the SM Higgs spectrum itself. Therefore, the mass of the Higgs boson remains as unspecified parameter in the SM theory. However, theoretical studies to determine constraints on the mass of the Higgs boson from considering triviality and vacuum stability lead to the upper and lower bounds on the mass, respectively [18]. 


\section{Upper bounds on the Higgs boson mass}

Due to the loop contributions from the Higgs boson self-interaction, the coupling of the Higgs field to gauge bosons fields and the top-Yukawa coupling, the Higgs coupling $\lambda$ changes with the effective energy scale as:

$$
\frac{d \lambda}{d t}=\frac{1}{16 \pi^{2}}\left[12 \lambda^{2}+6 \lambda g_{t}^{2}-3 g_{t}^{4}-\frac{3}{2} \lambda\left(3 g^{2}+g^{\prime 2}\right)+\frac{3}{16}\left(2 g^{4}+\left(g^{2}+g^{\prime 2}\right)^{2}\right)\right],
$$

where $t \equiv \log \frac{Q^{2}}{Q_{0}^{2}}$ with $Q_{0}$ being some reference scale, and $g_{t} \equiv \frac{\sqrt{2} M_{t}}{v}$ being the top-Yukawa coupling. Neglecting all coupling other than the Higgs boson self-coupling, Eqn. (2.84) becomes:

$$
\frac{d \lambda}{d t}=\frac{3 \lambda^{2}}{4 \pi^{2}}
$$

Taking the reference scale $Q_{0}$ to be the vacuum expectation value $v$ and solving Eqn. (2.85), we obtain the evolution equation for $\lambda$ of the form:

$$
\begin{aligned}
\frac{1}{\lambda(Q)} & =\frac{1}{\lambda(v)}-\frac{3}{4 \pi^{2}}, \log \left(\frac{Q^{2}}{v^{2}}\right) \\
\frac{1}{\lambda(Q)} & =\frac{1}{\lambda(v)-\frac{3 \lambda(v)}{4 \pi^{2}} \log \left(\frac{Q^{2}}{v^{2}}\right)} .
\end{aligned}
$$

The triviality refers to the fact that if we are allowed to take $Q \rightarrow \infty$ keeping $\lambda(Q)>0$ as a requirement for the stability of the theory, the coupling $\lambda(v)$ at low energy is driven to 0 , which implies a trivial, non-interacting theory. However, at lager and larger scales of $Q$, it is more and more likely that new physics, beyond the Standard Model, will appear and the possible triviality problem connected to pure scalar theory can be avoided.

Consider a high energy scale $\Lambda$ at which the new physics enters in and the Standard Model is embedded in a more complete theory. Then $\Lambda$ acts as a cutoff energy scale for the effective Standard Model theory. Depending on the specific value of $\Lambda$, an upper bound on the Higgs mass $M_{H}$ can be derived [19]. The cutoff scale $\Lambda$ is presumably smaller than the Plank scale $\left(\approx 10^{19} \mathrm{GeV}\right)$ and also smaller than the $\Lambda_{N P}$ energy scale at which the perturbative theory breaks down and non-perturbative effects become important.

To obtain the upper bound on $M_{H}$, we require the running Higgs coupling $\lambda(Q)$ be finite at the cutoff scale $\Lambda$ :

$$
\frac{1}{\lambda(\Lambda)}>0
$$

From Eqns. (2.63), (2.86), and (2.87), the approximate upper bound on the Higgs boson mass is evaluated by:

$$
M_{H}^{2}<\frac{8 \pi^{2} v^{2}}{3 \log \left(\Lambda^{2} / v^{2}\right)}
$$

Suppose there is no new physics before the grand unification scale and set $\Lambda \sim 10^{16} \mathrm{GeV}$, 
the upper bound on the Higgs boson mass is then estimated as:

$$
M_{H}<160 \mathrm{GeV}
$$

It can be seen from Eqn. (2.87) that as the cutoff scale $\Lambda$ becomes smaller, the constraint on the Higgs mass upper bound becomes progressively loose. For example, with $\Lambda \sim 3 \mathrm{TeV}$, the upper bound is roughly $600 \mathrm{GeV}$.

Considering the contribution of the top quark and gauge bosons to the evolution equation for $\lambda$, but just focusing on the case of a heavy Higgs boson, which corresponds to $\lambda>$ $g_{t}, g, g^{\prime}$, the equation of running $\lambda$ (Eqn. (2.84)) is simplified as:

$$
\frac{d \lambda}{d t} \sim \frac{\lambda}{16 \pi^{2}}\left[12 \lambda+6 g_{t}^{2}-\frac{3}{2}\left(3 g^{2}+g^{\prime 2}\right)\right]
$$

Following a similar analysis applied for the pure scalar field theory and requiring the running coupling $\lambda(\Lambda)$ to be finite up to the scale $\Lambda$, a more stringent upper limit on the Higgs boson mass is obtained as a function of top quark mass $M_{t}$. The upper curve in Fig. 2.3 shows the upper bound on the Higgs mass as a function of the cutoff scale $\Lambda$ [19]. The numerical value for the bound is calculated with considering the evolution of the gauge coupling constants and top-Yukawa coupling. The upper filled area indicates the sum of theoretical uncertainties in the $M_{H}$ upper bound when keeping top quark mass fixed at $M_{t}=175 \mathrm{GeV}$. The cross-hatched area shows the additional uncertainty when varying $M_{t}$ from 150 to $200 \mathrm{GeV}$.

\section{Lower bound on the Higgs boson mass}

The lower bound on the Higgs boson mass is derived from requiring the spontaneous symmetry-breaking minimum to be an absolute minimum of the effective Higgs potential up to some cutoff scale $\Lambda$. This requirement, referred to as vacuum stability, is essentially equivalent to requiring the Higgs running coupling $\lambda$ to remain positive at all scales up to a cutoff point $\Lambda$.

At the limit where the Higgs boson mass is small (corresponding to small $\lambda$ ), the evolution equation for $\lambda$ in Eqn. (2.84) becomes:

$$
\frac{d \lambda}{d t}=\frac{1}{16 \pi^{2}}\left[-3 g_{t}^{4}+\frac{3}{16}\left(2 g^{4}+\left(g^{2}+g^{\prime 2}\right)^{2}\right)\right]
$$

The solution to Eqn. (2.91) at scale $\Lambda$ is written as:

$$
\lambda(\Lambda)=\lambda(v)+\frac{1}{16 \pi^{2}}\left[-3 g_{t}^{4}+\frac{3}{16}\left(2 g^{4}+\left(g^{2}+g^{\prime 2}\right)^{2}\right)\right] \log \left(\frac{\Lambda^{2}}{v^{2}}\right)
$$

Imposing the requirement of vacuum stability $(\lambda(\Lambda)>0)$ on Eqn. (2.92) and using the relation in (2.63), we get the lower bound on the Higgs boson mass as a function of top 


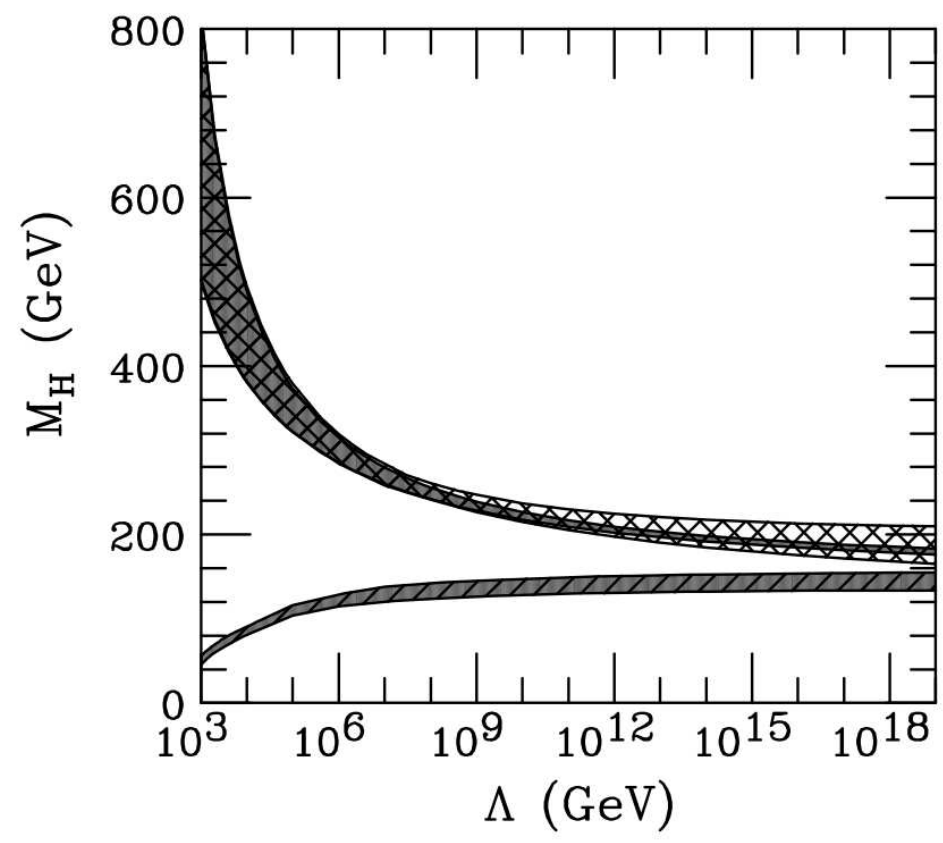

Figure 2.3: The theoritical upper and lower bounds on the Higgs boson mass

quark mass and the scale $\Lambda$ :

$$
M_{h}^{2}>\frac{v^{2}}{8 \pi^{2}}\left[-3 g_{t}^{4}+\frac{3}{16}\left(2 g^{4}+\left(g^{2}+g^{\prime 2}\right)^{2}\right)\right] \log \left(\frac{\Lambda^{2}}{v^{2}}\right)
$$

A similar analysis procedure as above has been carried out using the two-loop normalization group [20], provide lower bounds at fixed $M_{t}=175 \mathrm{GeV}$ and $\alpha_{s}\left(M_{Z}\right)=0.118$ as shown by the lower curve in Fig. 2.3. The solid area around this curve indicates the theoretical uncertainty. The lower bound on $M_{H}$ is sensitive to $\Lambda$ and gets weaker as $\Lambda$ get smaller. If the Standard Model is valid up to an energy scale of $10^{16} \mathrm{GeV}$, the lower limit on $M_{H}$ is about $130 \mathrm{GeV}$. But if the new physics scale appears at $\approx 1 \mathrm{TeV}$, it goes down to $\approx 70 \mathrm{GeV}$.

\subsubsection{Indirect Searches for the SM Higgs boson}

Consistency of the SM framework requires that all measurements are accommodated by the same values of SM parameters as the coupling constants of various interactions and the masses of the fundamental fermions, vector bosons and the Higgs boson. Based on this requirement, a stringent constraint on $M_{H}$ is obtained by performing a fit to a set of precision electroweak data to minimize a $\chi^{2}$ [21] calculated by comparing electroweak observables, their errors and correlations with the predictions calculated in the SM. 
To achive the best precision, a combination of the hadronic vacuum polarization, $14 Z$ pole results obtained at the electron-proton colliders LEP and SLC, as well as three direct results measured in high- $Q^{2}$ interactions at the Tevatron namely top quark mass, $W$ boson mass and $W$ boson width have been used for the fit to derive the constraint on the mass of the Higgs boson [2]. The combined direct measurement results of top quark mass, $W$ boson mass and $W$ boson width from the Tevatron experiments CDF and $\mathrm{D} \varnothing$ are :

$$
\begin{aligned}
M_{t} & =173.20 \pm 0.87(\mathrm{GeV}) \\
M_{W} & =80.385 \pm 0.015(\mathrm{GeV}) \\
\Gamma_{W} & =2.046 \pm 0.049(\mathrm{GeV})
\end{aligned}
$$

Including above high- $Q^{2}$ measurement results in the fit, the observed value of $\Delta \chi^{2}\left(M_{H}\right)=$ $\chi_{\min }^{2}\left(M_{H}\right)-\chi_{\min }^{2}$ is obtained as a function of $M_{H}$ and represented by the solid line in Fig. 2.4 [1]. The theoretical uncertainty in the SM calculation due to missing higher-order electroweak, strong and mixed corrections is estimated by ZFITTER [21] and presented by the thickness of the shaded curve. The preferred value for the mass of the SM Higgs boson, corresponding to the minimum of the $\Delta \chi^{2}\left(M_{H}\right)$ curve, is at $94 \mathrm{GeV}$. An experimental uncertainty is derived from setting $\Delta \chi^{2}\left(M_{H}\right)=1$, and according to the fit result, the mass of the SM Higgs boson should be in the range $M_{H}=94_{-24}^{+29} \mathrm{GeV}$ at $68 \%$ confidence level.

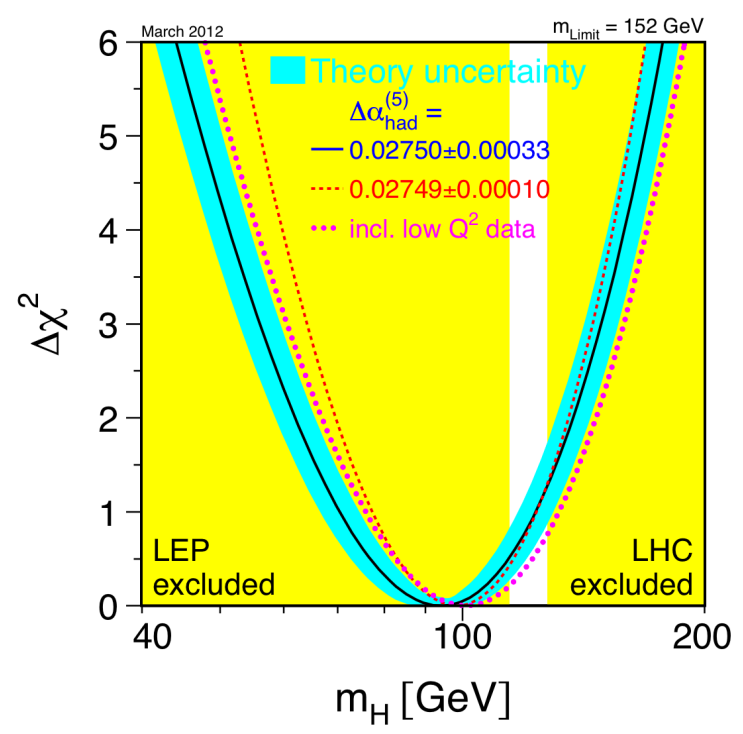

Figure 2.4: Constraints on the Higgs boson mass from precision electroweak data. The black solid line presents the result of the fit $\Delta \chi^{2}\left(M_{H}\right)=\chi_{\min }^{2}\left(M_{H}\right)-\chi_{\min }^{2}$ as a function of $M_{H}$. The associated band represents the estimated theory-uncertainty due to missing higher-order corrections. The vertical bands show the $95 \%$ confidence level exclusion limits on $M_{H}$ derived from the direct searches at LEP, Tevatron and LHC [1]. 
While the fit result is not a proof that the SM Higgs boson actually exists, it does serve as a guideline the preferred mass range to focus the searches. Including both the theoretical and experimental errors, the one-sided 95\% confident level upper limit on $M_{H}$ given at $\Delta \chi^{2}\left(M_{H}\right)=2.7$ (taking the theory-uncertanty shaded band into account) is $152 \mathrm{GeV}$. The upper limit $M_{H}<152 \mathrm{GeV}$ is clearly consistent with the $95 \%$ C.L. lower limit on $M_{H}$ obtained from the direct search performed at LEP as well as the 95\% C.L. exclusion regions obtained from direct searches for Higgs boson performed at the LHC and Tevatron as described in the next two sections.

\subsubsection{Direct Searches for the SM Higgs Boson}

\section{Decays of the SM Higgs Boson}

Since the Higgs boson was incorporated into the Standard Model, phenomenological studies have been carried out to predict the decay branching ratios of the Higgs boson in terms of its unknown mass. Decays to fermions and weak gauge bosons $(H \rightarrow f \bar{f}, H \rightarrow W W$ and $H \rightarrow Z Z$ ) are possible at tree level, while the decays $H \rightarrow \gamma \gamma, g g$ occur at the one-loop level.

\section{- Decay to fermion pairs}

The fermionic decays are dominant for the Higgs boson with mass below the $W^{+} W^{-}$threshold. Since the couplings of the Higgs boson to fermions are proportional to fermion mass $M_{f}$, the partial width of Higgs boson into any pair of charged leptons or quarks is proportional to $M_{f}^{2}$ :

$$
\Gamma(h \rightarrow f \bar{f})=\frac{g^{2} N_{c} M_{f}^{2}}{32 \pi M_{W}^{2}} M_{H} \beta^{3}
$$

where $N_{c}$ is 1 for charged leptons and 3 for quarks, $\beta \equiv \sqrt{1-4 M_{f}^{2} / M_{H}^{2}}$. The one-loop electroweak radiation corrections to decays into fermion can approach $10 \%$ at $M_{H} \approx 1 \mathrm{TeV}$, but are rather small for the mass range where the decays in $f \bar{f}$ final states are substantial. These corrections to decays into a pair of quarks $(H \rightarrow q \bar{q})$ can be neglected in comparison with significant QCD corrections.

The QCD corrected decay width into quarks shown in Ref. [22] has been expressed in terms of running quark mass $M_{q}\left(M_{H}\right)$ and running strong coupling $\alpha\left(M_{H}\right)$ evaluated at the energy scale of $M_{H}^{2}$ as:

$$
\Gamma(h \rightarrow q \bar{q})=\frac{3 g^{2}}{32 \pi M_{W}^{2}} M_{q}^{2}\left(M_{H}^{2}\right) M_{H} \beta^{3}\left(1+5.67 \frac{\alpha_{s}\left(M_{H}^{2}\right)}{\pi}+\cdots\right)
$$

It is clear that the Higgs boson preferably decays into the heaviest fermion kinematically allowed.

The branching ratios with QCD corrections for the dominant decay modes to pairs of fermions are computed using the program HDECAY and shown in Fig. 2.5. For mass 
hypotheses in the range $10 \mathrm{GeV}<M_{H}<160 \mathrm{GeV}$, where $m_{t}>M_{H} / 2$, the $H \rightarrow b \bar{b}$ is the most important $f \bar{f}$ decay mode.

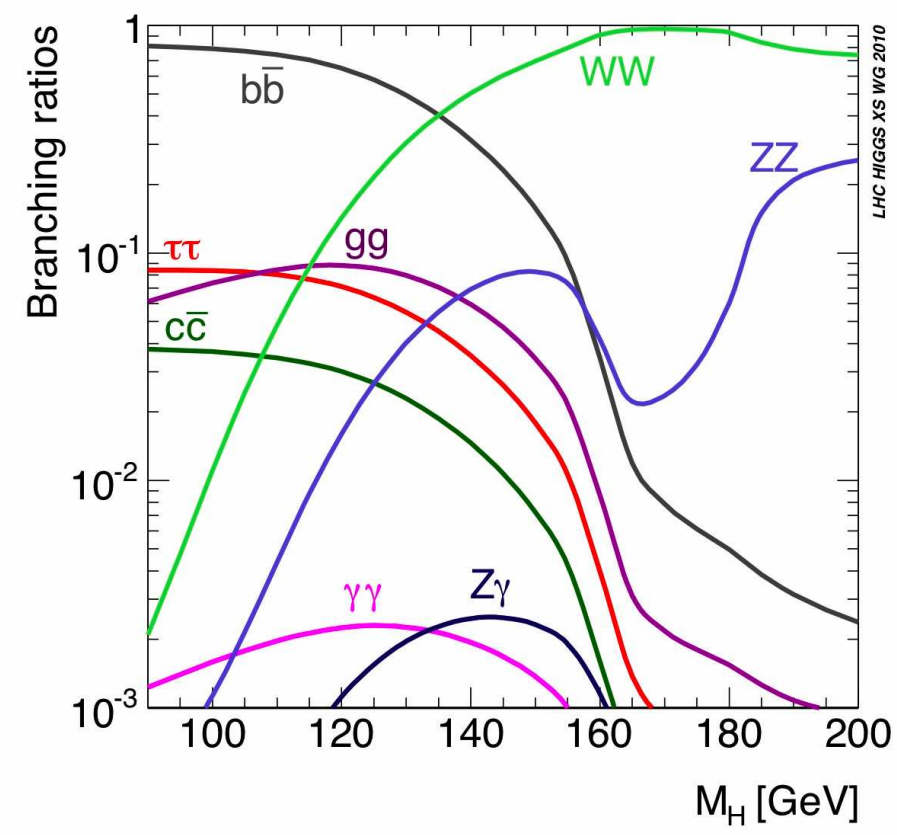

Figure 2.5: Branching ratios of the SM Higgs boson.

\section{- Decay to boson pairs}

With mass in the range $2 M_{W}<M_{H}<600 \mathrm{GeV}$, the Higgs boson will decay preferentially into a pair of weak gauge bosons, $H \rightarrow V V\left(V=W^{ \pm}, Z\right)$. A perturbative estimate should be reliable in this mass range, and the decay widths of the Higgs boson into physical pairs of $W^{+} W^{-}$and $Z Z$ were found in [23] to be:

$$
\begin{aligned}
\Gamma\left(h \rightarrow W^{+} W^{-}\right) & =\frac{g^{2}}{64 \pi} \frac{M_{H}^{3}}{M_{W}^{2}} \sqrt{1-r_{W}}\left(1-r_{W}+\frac{3}{4} r_{W}^{2}\right), \\
\Gamma(h \rightarrow Z Z) & =\frac{g^{2}}{128 \pi} \frac{M_{H}^{3}}{M_{W}^{2}} \sqrt{1-r_{Z}}\left(1-r_{Z}+\frac{3}{4} r_{Z}^{2}\right),
\end{aligned}
$$

where $r_{V} \equiv 4 M_{V}^{2} / M_{h}^{2}$.

With an intermediate mass, $M_{W}<M_{H}<2 M_{W}$, the Higgs boson can also decay to a pair of vector bosons $H \rightarrow V V^{*}$ where one boson $V^{*}$ is virtual. The inclusive rates $\Gamma\left(H \rightarrow V V^{*}\right)$ for all available channels $V^{*} \rightarrow f \bar{f}$ are given by [24]:

$$
\begin{aligned}
\Gamma\left(H \rightarrow W W^{*}\right) & =\frac{3 g^{4} M_{H}}{512 \pi^{3}} F\left(\frac{M_{W}}{M_{H}}\right) \\
\Gamma\left(h \rightarrow Z Z^{*}\right) & =\frac{g^{4} M_{H}}{2048 \pi^{3} \cos ^{4} \theta_{W}}\left(7-\frac{40}{3} \sin ^{2} \theta_{W}+\frac{160}{9} \sin ^{4} \theta_{W}\right) F\left(\frac{M_{Z}}{M_{H}}\right),
\end{aligned}
$$


where

$$
\begin{aligned}
F(x) \equiv & -\left|1-x^{2}\right|\left(\frac{47}{2} x^{2}-\frac{13}{2}+\frac{1}{x^{2}}\right)-3\left(1-6 x^{2}+4 x^{4}\right)|\ln (x)| \\
& +\frac{3\left(1-8 x^{2}+20 x^{4}\right)}{\sqrt{4 x^{2}-1}} \cos ^{-1}\left(\frac{3 x^{2}-1}{2 x^{3}}\right) .
\end{aligned}
$$

The branching ratios of $H \rightarrow W W$ and $H \rightarrow Z Z$ are plotted in Fig. 2.5. Both decay modes become significant (>10\%) for $M_{H}$ above $120 \mathrm{GeV}$.

The decay of Higgs boson to a pair of photons arises through fermion loops and vector boson $W^{ \pm}$loops as shown in Fig. 2.6.

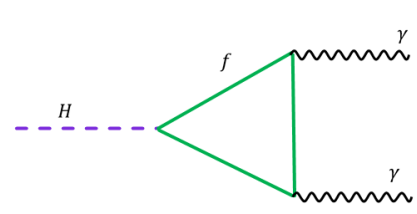

(a)

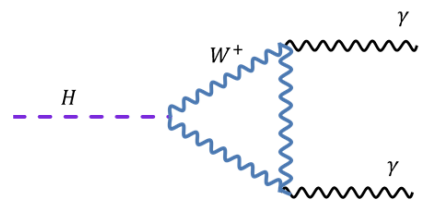

(b)

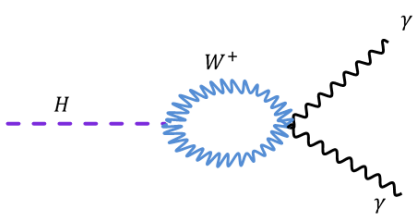

(c)

Figure 2.6: Diagrams contributing to $\boldsymbol{H} \rightarrow \boldsymbol{\gamma} \gamma$

At the lowest order, the decay width of $H \rightarrow \gamma \gamma$ is given by [25]:

$$
\Gamma(H \rightarrow \gamma \gamma)=\frac{\alpha^{2} g^{2}}{1024 \pi^{3}} \frac{M_{H}^{3}}{M_{W}^{2}}\left|\sum_{i} N_{c i} Q_{i} F_{i}\left(\tau_{i}\right)\right|^{2},
$$

where the sum is over fermions and $W^{ \pm}$bosons, $N_{c i}$ is the color multiplicity of particle $i$ $\left(N_{c i}=3\right.$ for quarks, $N_{c i}=1$ for leptons and $\left.W^{ \pm}\right), Q_{i}$ is the electric charge of particle $i$ in units of $e, \tau_{i} \equiv 4 M_{i}^{2} / M_{h}^{H}$, and $F_{i}\left(\tau_{i}\right)$ is defined differently for fermions and $W^{ \pm}$boson:

$$
\begin{aligned}
F_{\text {fermions }}\left(\tau_{i}\right) & =-2 \tau_{i}\left[1+\left(1-\tau_{i}\right) f\left(\tau_{i}\right)\right] \\
F_{W}\left(\tau_{i}\right) & =2+3 \tau_{i}\left[1+\left(2-\tau_{i}\right) f\left(\tau_{i}\right)\right] .
\end{aligned}
$$

The function $f\left(\tau_{i}\right)$ is given by:

$$
f\left(\tau_{i}\right)= \begin{cases}{\left[\sin ^{-1}\left(\sqrt{1 / \tau_{q}}\right)\right]^{2},} & \text { if } \tau_{i} \geq 1 \\ -\frac{1}{4}\left[\log \left(\frac{x_{+}}{x_{-}}\right)-i \pi\right]^{2}, & \text { if } \tau_{i}<1\end{cases}
$$

with

$$
x_{ \pm}=1 \pm \sqrt{1-\tau_{i}} .
$$

As shown in Fig. 2.5, the branching ratio of $H \rightarrow \gamma \gamma$ is increasing with Higgs mass for the lower mass region and reaches its maximum for $M_{H} \sim 125 \mathrm{GeV}$. For Higgs boson mass above $125 \mathrm{GeV}$, it becomes suppressed due to the rapidly increase of the $H \rightarrow V V$ decay 
modes.

The decay of the Higgs boson to gluons at one-loop level is similar to the decay to photons described above, with contributions from quark loops only. The decay width $\Gamma(H \rightarrow g g)$ is given by [26]:

$$
\Gamma(H \rightarrow g g)=\frac{\alpha_{s}^{2} g^{2}}{128 \pi^{3}} \frac{M_{H}^{3}}{M_{W}^{2}}\left|\sum_{q} \tau_{q}\left[1+\left(2-\tau_{q}\right) f\left(\tau_{q}\right)\right]\right|^{2},
$$

where $\tau_{q} \equiv 4 m_{q}^{2} / M_{H}^{2}$ and $f\left(\tau_{q}\right)$ is defined by 2.101 .

In the limit that the quark mass is much less than the Higgs boson mass, we have:

$$
\tau_{q}\left[1+\left(2-\tau_{q}\right) f\left(\tau_{q}\right)\right] \rightarrow \frac{m_{q}^{2}}{M_{H}^{2}} \log ^{2}\left(\frac{M_{q}}{M_{h}}\right)
$$

Therefore, the contribution of light quark loops to the decay mode $H \rightarrow g g$ can be neglected, and the dominant contribution to the decay width to gluons is from the top quark loop.

\section{The SM Higgs production and searches at LEP}

The Large Electron Positron collider (LEP) at CERN colliding began operation in 1989 at center-of-mass energy $\sqrt{s}=90 \mathrm{GeV}$ and was eventually upgraded to $\sqrt{s} \sim 209 \mathrm{GeV}$ (LEP2) at the end of its run in the year of 2000. Due to the smallness of the Higgs boson coupling to an electron-positron pair, the SM Higgs boson is expected to be produced at LEP mainly in association with the $\mathrm{Z}$ boson through the associated process $e^{+} e^{-} \rightarrow Z^{*} \rightarrow Z H$, as shown in Fig. 2.7.

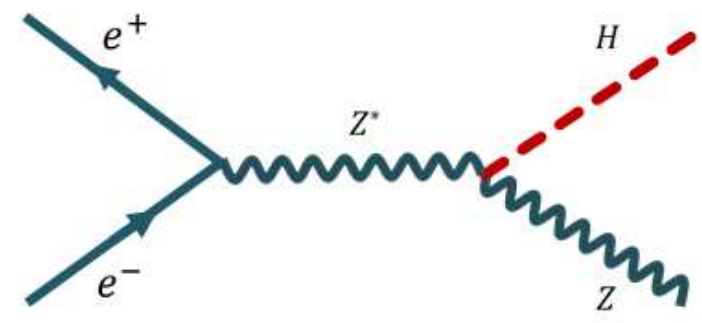

Figure 2.7: Feynman diagram of $\boldsymbol{e}^{+} \boldsymbol{e}^{-} \rightarrow Z^{*} \rightarrow \boldsymbol{Z} \boldsymbol{H}$ production

For the range of Higgs boson mass which is relevant at LEP, the SM Higgs boson decays mostly to a $b \bar{b}$ pair. The main searches for the SM Higgs boson at LEP encompass final state topologies having four fermions [2]:

- The four-jet final state: $e^{+} e^{-} \rightarrow Z H \rightarrow q \bar{q} b \bar{b}$ where $H \rightarrow b \bar{b}$ and $Z \rightarrow q \bar{q}$

- The missing energy final state: $e^{+} e^{-} \rightarrow Z H \rightarrow \nu \bar{\nu} b \bar{b}$ where $H \rightarrow b \bar{b}$ and $Z \rightarrow \nu \bar{\nu}$

- The leptonic final state: $e^{+} e^{-} \rightarrow Z H \rightarrow l^{+} l^{-} b \bar{b}$ where $H \rightarrow b \bar{b}$ and $Z \rightarrow l^{+} l^{-}$ ( $l$ denotes an electron or a muon) 
- The tau lepton final state: $e^{+} e^{-} \rightarrow Z H \rightarrow \tau \bar{\tau} b \bar{b}$ where $H \rightarrow b \bar{b}$ and $Z \rightarrow \tau \bar{\tau}$ or $H \rightarrow \tau \bar{\tau}$ and $Z \rightarrow b \bar{b}$

Using an $e^{+} e^{-}$collision data set of $2.6 \mathrm{fb}^{-1}$ at centre-of-mass energy between 189 and $209 \mathrm{GeV}$, the LEP Collaborations established a lower bound of $114.4 \mathrm{GeV}$ on the SM Higgs boson at the $95 \%$ confidence level [2].

\section{SM Higgs production at hadron colliders}

There have been two major hadron colliders have been operating in recent years, the CERN Large Hadron Collider (LHC) and the Tevatron. The LHC, a $p p$ collider, is the highest energy accelerator available today with the $p p$ center-of-mass energy settled to $8 \mathrm{TeV}$. The Tevatron, a $p \bar{p}$ collider at Fermi Nationsl Accelerator Laboratory (Fermilab), started producing collisions in 1988 at center-of-mass energy $\sqrt{s}=1.8 \mathrm{GeV}$ and was upgraded to have collider energy of $\sqrt{s}=1.96 \mathrm{GeV}$ in 2001, then ran up to September 2011. The main production mechanisms of the SM Higgs boson at hadron colliders are determined from the fact that the SM Higgs boson couples preferentially to heavy particles and there is high gluon luminosity at these colliders. The four main production processes for a SM Higgs boson in hadronic colliders are the gluon-gluon fusion mechanism, the weak vector boson fusion processes, the associated production with $W / Z$ bosons, and the associated production with top quarks or bottom quarks [27]. The Feynman diagrams of these processes are displayed in Fig. 2.8. Production rates of the SM Higgs boson for various production modes at the Tevatron and the LHC are summarized in Fig. 2.9 [28].

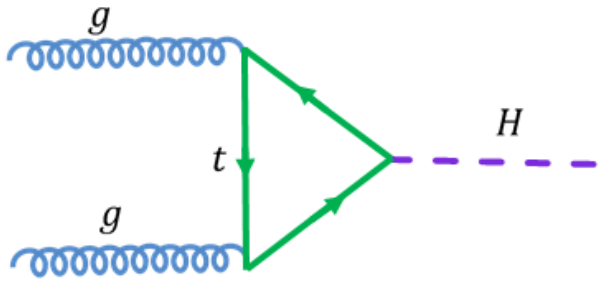

(a) Gluon fusion

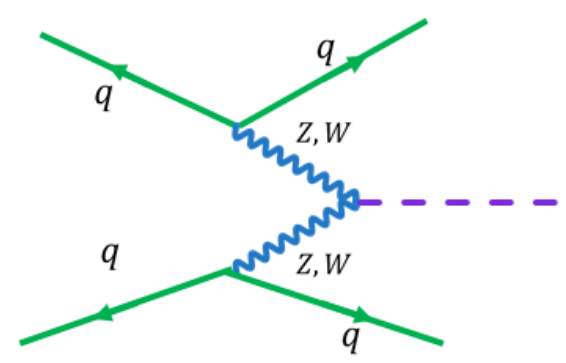

(c) Vector boson fusion

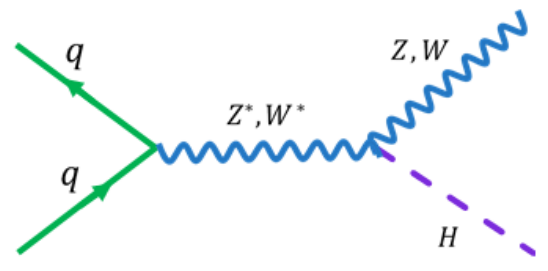

(b) Associated production with $\mathrm{W} / \mathrm{Z}$ bosons

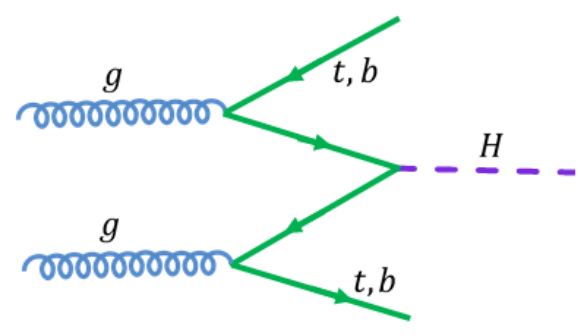

(d) Associated production with heavy quarks

Figure 2.8: The main SM Higgs boson production processes at hadron colliders. 

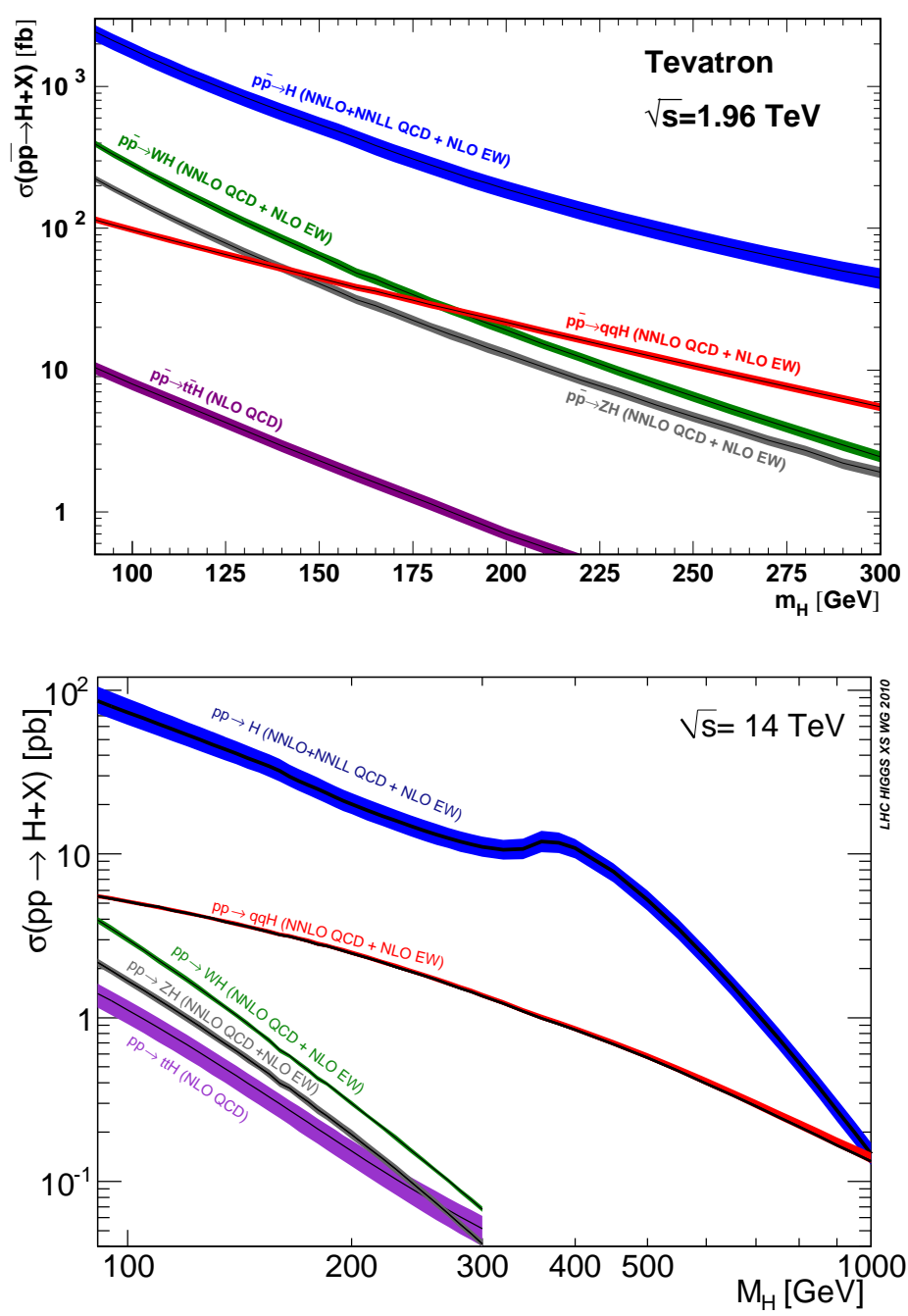

Figure 2.9: The production cross section for a SM Higgs boson at the Tevatron (top) and LHC (bottom).

\section{- Gluon-gluon fusion $g g \rightarrow H$}

The primary production mode of the SM Higgs boson at the Tevatron and the LHC is via gluon-gluon fusion mediated by a virtual heavy quark loop. The cross section for $g g \rightarrow H$ is a factor of 10 larger than all other production mode cross sections [29]. Radiative QCD corrections to the gluon-gluon fusion process are very important. The cross sections of this mode plotted in Fig 2.8 are computed at NNLO in QCD and include soft-gluon resummation effects at NNLO.

- Associated Higgs production with $W / Z$ bosons $q q \rightarrow V+H$

The associated production of a Higgs boson with a massive gauge boson $\mathrm{V}(V=W, Z)$ is an important channel in the low $M_{H}$ region at the Tevatron. It utilizes the leptonic decay of the $W / Z$ boson and the $H \rightarrow b \bar{b}$ decay mode to reject background. The 
production rate of $q q \rightarrow V+H$ mode is computed at NNLO in QCD and NLO in electroweak approximation, and ranges between $0.3 \mathrm{pb}$ and $3 \mathrm{pb}$ depending on the Higgs boson mass [29].

- Vector boson fusion $q q \rightarrow V V^{*} \rightarrow q q+H$

In the vector boson fusion (VBF) production mode, the Higgs boson is produced in association with two forward jets and its decay products are found in a central rapidity region, which allows to efficient reduction of background if suitable event selections are chosen. The production rate of the VBF mode is computed with NLO QCD corrections. It is one order of magnitude lower than production rate of the gluon-gluon fusion mode, but VBF is an important channel for measurements of the Higgs boson couplings and CP properties [29].

- Associated Higgs boson production with heavy quarks $g g, q q \rightarrow Q \bar{Q}+\boldsymbol{H}$ The plotted production rates of associated Higgs boson production with heavy quarks in Fig. 2.8 are computed at different levels of perturbative corrections [30]: at NLO in QCD for the $t \bar{t}$ associated production $(g g, q q \rightarrow t \bar{t}+H)$, and at the LO in QCD for the single-top associated production $(q q \rightarrow b t+H)$. Since any Higgs boson decay products in this channel would be present in the top decays, the large backgrounds (particularly from $t \bar{t} b \bar{b}$ and $t \bar{t} j j$ ) would make the observation of a Higgs boson signal from this production mode very difficult.

\section{Direct searches for the SM Higgs boson at Hadron Colliders}

The search for the SM Higgs boson is a central part of the Tevatron's physics program and a primary scientific goal of the LHC. After the shutdown of LEP in 2001, the CDF and D $\varnothing$ experiments at the Tevatron took the lead in Higgs searches and have performed searches in both low mass $\left(M_{H} \leq 130 \mathrm{GeV}\right)$ and high mass $\left(M_{H}>130 \mathrm{GeV}\right)$ regions. The SM Higgs analyses carried out in this thesis use the total $\sim 10 \mathrm{fb}^{-1}$ data set of $p \bar{p}$ collision at the center-of-mass energy $\sqrt{s}=1.96 \mathrm{TeV}$ accumulated by the $\mathrm{D} \varnothing$ experiment.

The two general purpose experiments at the LHC, ATLAS and CMS, have been constructed to cover a large spectrum of possible signatures in the LHC environment. The search for the SM Higgs boson has been one of the major guides to define the detector requirements and performances for these experiments [27]. The LHC started producing $p p$ collisions of the center-of-mass energy $\sqrt{s}=7 \mathrm{TeV}$ from March 2010, and then collider energy was increased to $8 \mathrm{TeV}$ in 2012. By June 2012, each experiment collected a data set

of $\sim 5.1 \mathrm{fb}^{-1}$ at the center-of-mass energy $\sqrt{s}=7 \mathrm{TeV}$ and an additional data set of above $\sim 5.1 \mathrm{fb}^{-1}$ at center-of-mass energy $\sqrt{s}=8 \mathrm{TeV}$.

The direct search for the SM Higgs boson at the hadron colliders is performed in five major decay modes, $H \rightarrow b \bar{b}, H \rightarrow \tau^{+} \tau^{-}, H \rightarrow Z Z, H \rightarrow W^{+} W^{-}$, and $H \rightarrow \gamma \gamma$.

\section{- $H \rightarrow b \bar{b}$ decay mode}


For Higgs boson mass below $130 \mathrm{GeV}$, the decay $H \rightarrow b \bar{b}$ has the largest branching ratio of the five search modes. However, the QCD production rate of bottom quarks at the hadron colliders is several orders of magnitude higher than the inclusive signal $H \rightarrow b \bar{b}$. Therefore, to suppress the QCD background, the $H \rightarrow b \bar{b}$ analysis search strategy focuses on the associated production of the Higgs boson with $\mathrm{W}$ or $\mathrm{Z}$ boson where the $\mathrm{W}$ or $\mathrm{Z}$ boson decays into a pair of leptons. Three exclusive subchannels corresponding to different leptonic decays of the vector boson are considered, $Z H \rightarrow$ $l^{+} l^{-} b \bar{b}, Z H \rightarrow \nu \bar{\nu} b \bar{b}$ and $W H \rightarrow l \nu b \bar{b}$. The $H \rightarrow b \bar{b}$ decay mode is the most important search channel at the Tevatron for the low $M_{H}$ region. The recent D $\varnothing$ and CDF combined search result based on the $H \rightarrow b \bar{b}$ decay mode shows an excess of events in the data compared to background prediction in the range $120<M_{H}<135 \mathrm{GeV}$ [31].

\section{- $H \rightarrow \tau^{+} \tau^{-}$decay mode}

For $M_{H} \lesssim 135 \mathrm{GeV}$, the decay $H \rightarrow \tau^{+} \tau^{-}$has an appreciable $(\simeq 8 \%)$ branching fraction. The search for the Higgs boson decaying into a pair of $\tau$ leptons has been carried out in independent channels corresponding to different decay modes of $\tau$ pairs, $e \mu, \mu \mu, e \tau_{h}, \mu \tau_{h}$ and $\tau_{h} \tau_{h}$ where the electrons and muons arise from leptonic $\tau$ decays and $\tau_{h}$ denotes hadronic $\tau$ decays $[32,33,34,35]$. Several Higgs boson production processes (associated production $W H, Z H$, vector boson fusion and gluon fusion) have been considered in this search, and classifying the events coming from different production processes based on jet multiplicity and charged lepton multiplicity of the final states improves the signal sensitivity. The seach for the $H \rightarrow \tau^{+} \tau^{-}$decay is the third most important analysis for low $M_{H}$ at the Tevatron.

\section{- $\boldsymbol{H} \rightarrow \gamma \gamma$ decay mode}

Despite its small branching fraction, the $H \rightarrow \gamma \gamma$ decay mode is an important discovery channel for a low mass Higgs at the LHC. The excellent resolution of the diphoton mass $M_{\gamma \gamma}$ makes the experimental signature of this decay very clean. The searches for $H \rightarrow \gamma \gamma$ decay by both CMS and ATLAS Collaborations indicate the presence of a significant excess in data compared to background prediction at around a mass point $M_{H}=125 \mathrm{GeV}$. The observed local $p$-values for a SM Higgs boson of mass $M_{H}=125 \mathrm{GeV}$ in CMS and $M_{H}=126.5 \mathrm{GeV}$ in ATLAS corresponding to above 4.0 Gaussian standard deviations $[32,36]$. The combined search result of D $\varnothing$ and CDF for $H \rightarrow \gamma \gamma$ also shows an excess of approximately two standard deviations at $M_{H}=125 \mathrm{GeV}$ and has a strong impact on Higgs boson coupling constraints [13].

\section{- $H \rightarrow Z Z$ decay mode}

The $H \rightarrow Z Z \rightarrow 4$ leptons decay is the golden channel for observing a Higgs boson at the LHC, producing a narrow four lepton mass peak on top of a small continuum background. Similar to the $H \rightarrow \gamma \gamma$ channel, the excellent mass resolution in the $H \rightarrow Z Z$ channel gives it a special role in the searches within the low mass region. 
Using the combined $7 \mathrm{TeV}$ and $8 \mathrm{TeV}$ data set, both CMS and ATLAS Collaborations have recently reported the presence of a significant excess in the Higgs mass range $120<M_{H}<130 \mathrm{GeV}$ in this channel $[32,36]$. The minimum of the observed local $p$-value occurred at $M_{H}=125.6 \mathrm{GeV}$ in the CMS search result for $H \rightarrow Z Z \rightarrow 4 l$ with a significance of 3.2 standard deviations. A similar result has been observed by the ATLAS collaboration with an observed local $p$-value of approximately 3.6 standard deviations.

- $\boldsymbol{H} \rightarrow W W$ decay mode

The $H \rightarrow W W$ decay mode contributes the majority of the signal sensitivity in the SM Higgs boson search for the mass region above $130 \mathrm{GeV}$. The dominant search channels related to this decay are $H \rightarrow W W \rightarrow l^{+} l^{-} \nu \bar{\nu}$, where both $\mathrm{W}$ bosons decay leptonically and $l$ denotes an electron or muon. The presence of neutrinos in the final states prevents precise reconstruction of the candidate $M_{H}$ and the mass resolution in this search is lower compared to $H \rightarrow \gamma \gamma$ and $H \rightarrow Z Z$ search channels. However, with the strong angular correlation between charged leptons in the final state, it is possible to extend the search sensitivity down to the $M_{H}$ as low as $120 \mathrm{GeV}$. A broad excess, which is consistent with a SM Higgs boson of mass $125 \mathrm{GeV}$, is observed at both the LHC and Tevatron. The Tevatron combined search results sho a one-totwo standard deviation excess in the region from 115 to $140 \mathrm{GeV}$ [13]. The observed significance for the SM Higgs boson of mass $125 \mathrm{GeV}$ is measured in CMS and ATLAS at 1.6 and 2.8 standard deviations, respectively [32, 36].

An additional search channel has been exploited at the DØ collaboration, $H \rightarrow$ $W W \rightarrow \ell \nu j j$ with one boson decaying leptonically and another hadronically. Details about $H \rightarrow W W \rightarrow \ell \nu j j$ analysis will be described in this thesis.

Combining direct search results from the ALEPH, DELPH, L3 and OPAL experiments at the LEP $e^{+} e^{-}$collider [2], the $\mathrm{D} \varnothing$ and CDF experiments at the Tevatron [37, 4], and the CMS [6] and ATLAS [5] experiments at the LHC limits the SM Higgs boson mass to $122<M_{H}<127 \mathrm{GeV}$ at $95 \%$ confidence level. Using a data set corresponding to integrated luminosities of $\sim 10.5 \mathrm{fb}^{-1}$ recorded at $\sqrt{s}=7 \mathrm{TeV}$ and $\sqrt{s}=8 \mathrm{TeV}$, the CMS and ATLAS Collaborations performed the search in the above five decay modes and each Collaboration reported an observation of a new particle consistent with the SM Higgs boson with a mass near $125 \mathrm{GeV}[32,36]$. The CDF and D $\varnothing$ Collaborations combined searches for the SM Higgs boson (including the searches described in this thesis) using data set of $\sim 10.5 \mathrm{fb}^{-1}$ recorded at $\sqrt{s}=1.96 \mathrm{TeV}$ reported a significant excess corresponding to 3.1 standard deviations at $M_{H}=125 \mathrm{GeV}$, consistent with the mass of the new particle observed at the LHC [13]. 


\subsection{Higgs Physics Beyond the Standard Model}

Any physics beyond the Standard Model that affects the properties of the Higgs boson of the Standard Model is of outmost importance to studies in particle physics. Two of the simplest kinds of new physics that have a major impact on the Higgs sector of the SM, which are considered in this dissertation, are the fourth generation of chiral matter $[38,39]$ and the Fermiophobic Higgs boson model [40].

\subsubsection{Fourth Generation of Fermions and Higgs Physics}

Inclusion of the fourth generation of fermions with masses larger than those of the three known generations is the most natural extension of the SM. The presence of the fourth generation of fermions would have a significant effect on the couplings of the Higgs boson to the SM particles and, therefore, modify both the production and decay properties of the Higgs boson. The contribution of two additional heavy fourth generation quarks to the quark-loop in $g g \rightarrow H$ production enhances the $g g \rightarrow H$ production cross section by a factor of 7.0 to 9.0, depending on the masses of the quarks and the Higgs boson [39, 41, 42]. Although the partial decay width for $H \rightarrow g g$ is enhanced by the same factor as the production cross section, $H \rightarrow V V$ decays continue to dominate over the loop-mediated decays for $M_{H}>135 \mathrm{GeV}$.

Under the assumption of a sequential fourth generation of fermions, constraints from precision electroweak data on the mass of the Higgs boson becomes less restrictive and the allowed mass range is expanded to $115<M_{H}<750 \mathrm{GeV}$ [39]. Previous direct searches for the Higgs boson within the context of the fourth generation at Tevatron have excluded at $95 \%$ C.L. the mass range $131<M_{H}<207 \mathrm{GeV}$ [4]. Similar searches performed at CMS [43] and ATLAS [44] have excluded $110<M_{H}<600 \mathrm{GeV}$ and $140<M_{H}<185 \mathrm{GeV}$. The SM boson Higgs searches carried out in this thesis are re-interpret in models containing a fourth generation of fermion and are combined to others at the CDF and D $\varnothing$ to make the latest exclusion reported by Tevatron, $121<M_{H}<232 \mathrm{GeV}$ [13].

\subsubsection{Fermiophobic Higgs Model}

The Fermiophobic Higgs model (FMH) is an extension of the SM where multiple Higgs bosons are allowed and the Higgs couplings are modified such that the Higgs bosons only couple to vector bosons at tree level $[40,45,46]$. Since a fermiophobic Higgs boson $\left(H_{F}\right)$ has zero tree-level couplings to fermions, gluon fusion production $g g \rightarrow H_{F}$ is suppressed to a negligible rate and direct decays of $H_{F}$ to fermions become impossible. The vector

boson fusion production and the associated production with $\mathrm{W} / \mathrm{Z}$ boson remain nearly unchanged relative to the SM, but the branching fractions for bosonic decays $H_{F} \rightarrow W W$, $H_{F} \rightarrow W W$ and $H_{F} \rightarrow \gamma \gamma$ increase significantly and comprise nearly the entire decay width. The $H_{F} \rightarrow \gamma \gamma$ decay is further enhanced over the SM since the negative interference 
between top quark and $\mathrm{W}$ loops responsible for this decay in SM is no longer present in the FHM. For the low mass region, the diphoton decay of a fermionphobic Higgs boson decay is enhanced by a magnitude and this decay mode provides most of search sensitivity for $M_{H_{F}} \sim 125 \mathrm{GeV}$.

Previous searches for a fermiophobic Higgs boson using the $H_{F} \rightarrow \gamma \gamma$ channel by CMS and ATLAS Collaborations excluded $M_{H_{F}}$ in the ranges $119<M_{H_{F}}<121 \mathrm{GeV}$ and $110<$ $M_{H_{F}}<118 \mathrm{GeV}$, respectively. Combining fermiophobic Higgs boson searches from multiple final states at Tevatron, including the searches performed in this thesis, has excluded signal with masses in the range $110<M_{H_{F}}<116 \mathrm{GeV}$ at $95 \%$ C.L. [13].

\subsection{Search for the SM Higgs Boson in Leptons plus Jets Final States}

Searching for the SM Higgs boson begins by looking for detector signatures that are consistent with its production and final decay products. This thesis is focused on searches in the final states containing leptons and jets, which have been carried out in two different physics analyses at the $\mathrm{D} \varnothing$ experiment, namely $Z H \rightarrow \ell \ell b b$ and $\ell \nu j j$ analyses. The searches are performed using a data set of proton-antiproton collisions corresponding to $9.7 \mathrm{fb}^{-1}$ of integrated luminosity collected with the $\mathrm{D} \varnothing$ detector at a center of mass energy of $\sqrt{s}=1.96 \mathrm{TeV}$.

\subsubsection{The SM Higgs Boson Signal in $Z H \rightarrow \ell \ell b b$ and $\ell \nu j j$ Analyses The SM Higss signal in $Z H \rightarrow \ell \ell b b$ analysis}

The $Z H \rightarrow \ell \ell b b$ analysis searches for the Higgs boson produced in association with a $\mathrm{Z}$ boson, with the $\mathrm{Z}$ decaying leptonically to a pair of $\mu^{+} \mu^{-}$or $e^{+} e^{-}$and the Higgs boson decaying to a pair of bottom and anti-bottom quarks. The leading order Feyman diagram for the $Z H \rightarrow \ell^{+} \ell^{-} b b$ signal process is shown in Fig. 2.10. Being more sensitive to the production of a low mass Higgs boson, the $Z H \rightarrow \ell \ell b b$ analysis performs the search for the mass range $90 \leq M_{H} \leq 150 \mathrm{GeV}$.

\section{The SM Higgs signal in the $\ell \nu j j$ channel.}

The $\ell \nu j j$ analysis performs the search for the SM Higgs boson using events containing one charge lepton $(\ell=e$ or $\mu)$, a significant imbalance in transverse energy $\left(E_{\mathrm{T}}\right)$ arising from a neutrino, and two or more jets. It comprises searches for multiple signal processes, $W H \rightarrow \ell \nu b b, H \rightarrow W W \rightarrow \ell \nu j j$, and $V H \rightarrow V W W \rightarrow \ell \nu j j j j$ (where $V=W$ or $Z)$. Leading order Feyman diagrams describing three main signal processes are shown in Fig. 2.11. In the $W H \rightarrow \ell \nu b \bar{b}$ process, the Higgs boson is produced via associated production with a $W$ boson, then the Higgs boson decays into a b-quark pair and the $W$ boson decays 


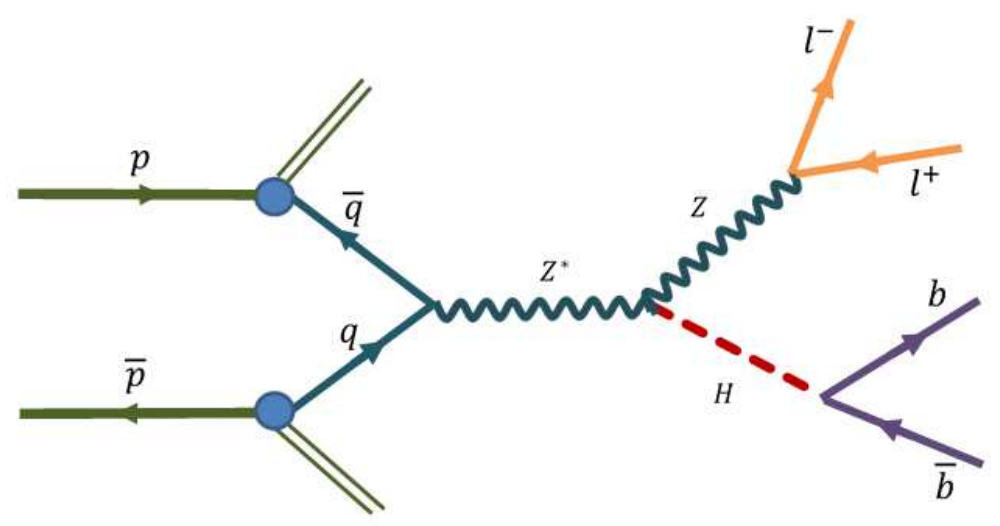

Figure 2.10: Feynman diagram of $\boldsymbol{Z} \boldsymbol{H} \rightarrow \boldsymbol{\ell} \ell \boldsymbol{b} \boldsymbol{b}$ signal.

into a charge lepton and a neutrino. The Higgs boson in the $H \rightarrow W W^{*} \rightarrow \ell \nu j j$ process is produced by either gluon-gluon fusion or VBF process and decays into a pair of $W$ bosons, then one $W$ boson decays to a charged lepton and an associated neutrino, the other $W$ boson decays to a quark-antiquark pair that hadronizes into two jets of particles. In the $V H \rightarrow$ $V W W \rightarrow \ell \nu j j j j$ process, the Higgs boson is produced via associated production with a weak boson $V$ and decays into a pair of $W$ bosons, and then one $W$ boson decays leptonically into a charged lepton and a neutrino, the other two weak bosons decays hadroncally into four jets. A small contribution to the Higgs boson sinal coming from $Z H$ production and from the decay $H \rightarrow Z Z$ when one of the charged leptons from the $Z \rightarrow \ell \ell$ decay is not identified in the detector is also consider in this analysis.

The three main Higgs boson production and decay channels considered in the $\ell \nu j j$ analysis are sensitive to different hypotheses for $M_{H}$, the $W H \rightarrow \ell \nu b b$ process is more sensitive to the low mass region, while the $H \rightarrow W W \rightarrow \ell \nu j j$ and $V H \rightarrow V W W \rightarrow \ell \nu j j j j$ processes are more sensitive to the high mass region, but contribute some senstivity at lower masses through decays piercing a virtual $W$ boson. Therefore, the $l \nu j j$ analysis is performed the search in the mass range $90 \leq M_{H} \leq 200 \mathrm{GeV}$.

\subsubsection{Background in $Z H \rightarrow \ell \ell b b$ and $\ell \nu j j$ Analyses}

Searching for the SM Higgs boson is challenging, because its primary decays are similar to prodigious backgrounds at hadron colliders. There are two types of background processes that are considered in $Z H \rightarrow \ell \ell b b$ and $\ell \nu j j$ analyses:

- The physics background processes, $W+$ jets, $Z+$ jets, top-quark pair, single-top and diboson productions, are simulated by Monte Carlo (MC).

- The instrumental background from multijet events is estimated through control samples in the data. 


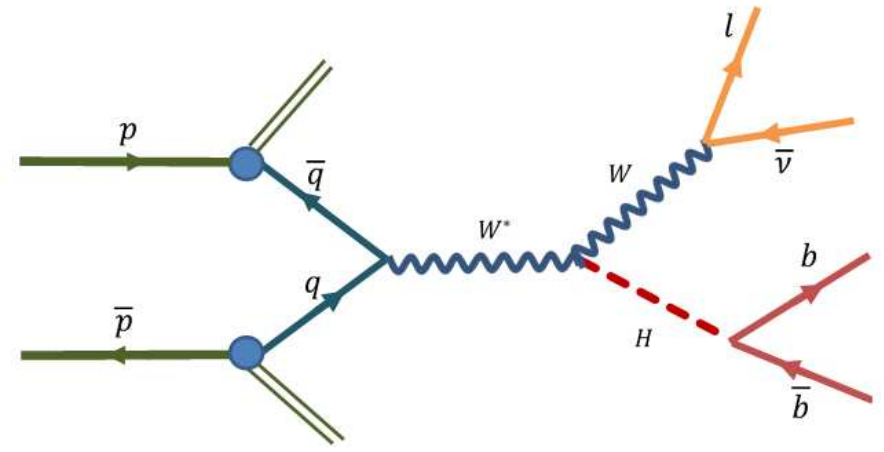

(a) $W H \rightarrow \ell \nu b \bar{b}$

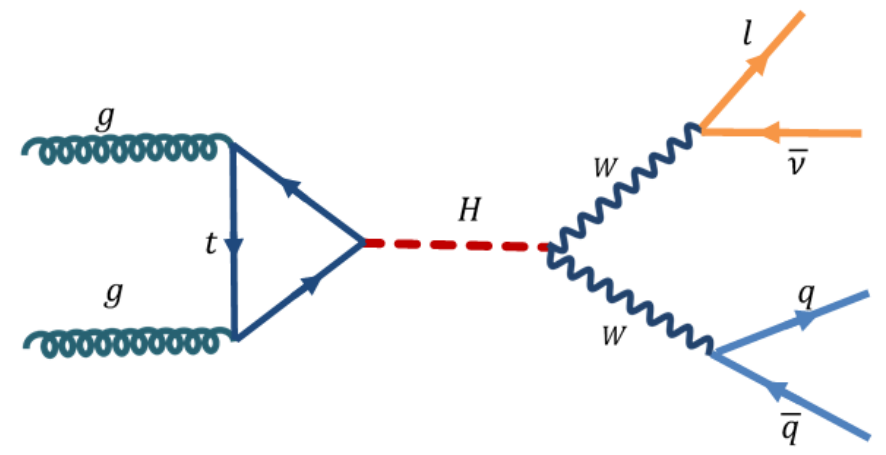

(b) $\boldsymbol{H} \rightarrow W W \rightarrow \ell \nu j j$

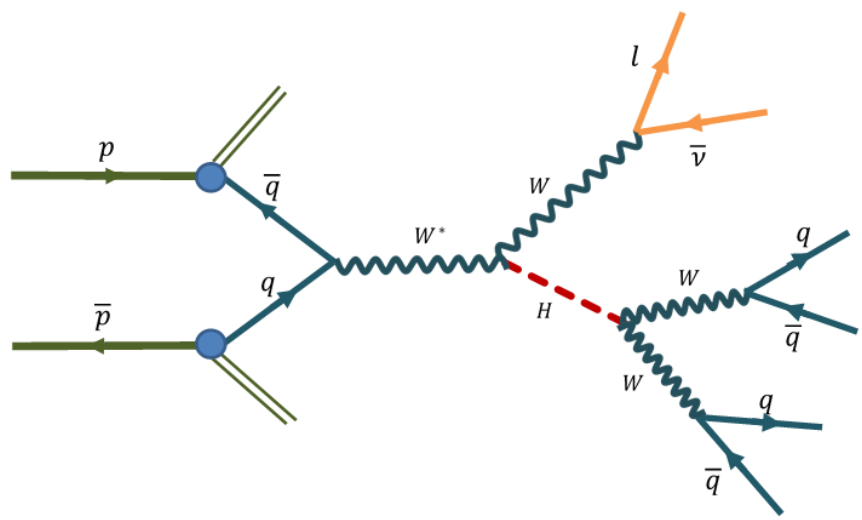

(c) $W H \rightarrow W W W \rightarrow \ell \nu j j j j$

Figure 2.11: Feyman diagrams of the main SM Higgs signals in $\boldsymbol{\ell} \boldsymbol{\nu} \boldsymbol{j} \boldsymbol{j}$ analyses. 
The $Z+$ jets, top-quark pair, diboson production and multijets are background processes contribute in both the $Z H \rightarrow \ell \ell b b$ and $\ell \nu j j$ analyses. For the $\ell \nu j j$ channel, the production of a $W$ boson in association with jets and single top production are considered as two dominated backgrounds.

\section{$W+$ jets and $Z+$ jets}

The largest contribution to the background in the $\ell \nu j j$ channel comes from $W+$ jets events where the $W$ boson is produced in association with quarks or gluons with the $W$ boson decaying leptonically and the quarks or gluons hadronizing into jets. The $W+$ jets sample can be split into $W+\mathrm{LF}$ and $W+\mathrm{HF}$ where the jets are initiated from light flavor quark $(u, d, s)$ and heavy flavor quarks $(c, b)$, respectively.

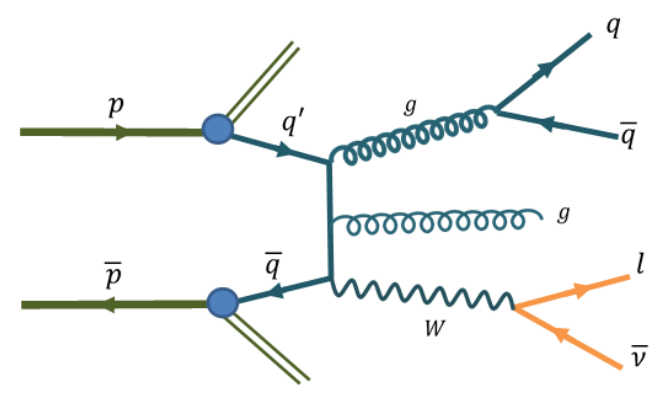

(a) $W+$ jets

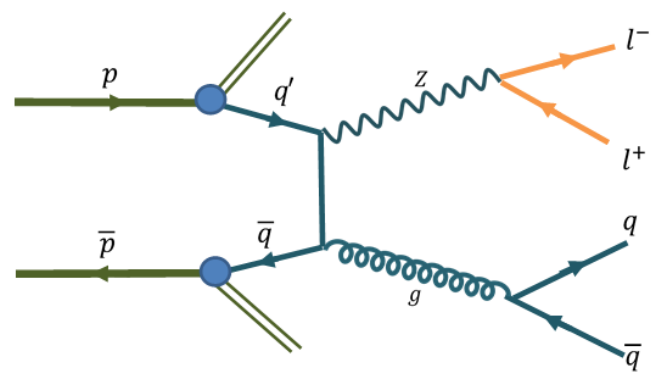

(b) $Z+$ jets

Figure 2.12: Feynman diagrams for $\boldsymbol{W}+$ jets and $\boldsymbol{Z}+$ jets background

The most dominant background in the $Z H \rightarrow \ell \ell b b$ channel arises from the $Z+$ jets process. Similar to $W+$ jets, the $Z+$ jets events are categorized in $Z+\mathrm{LF}$ and $Z+\mathrm{HF}$. The $Z+$ jets events, where one charged lepton from $Z$ boson decay is lost to detection, can also mimic expected signal signatures in the $\ell \nu j j$ channel. In fact, the second largest background in the $\ell \nu j j$ channel comes from those $Z+$ jets events. Feyman diagrams shown in Fig. 2.12 are examples for $W+$ jets and $Z+$ jets processes.

\section{Top Pair Production}

A leading order Feyman diagram of top pair production $(t \bar{t})$ is shown in Fig 2.13. The $t \bar{t}$ events can be background candidates for the $Z H \rightarrow \ell \ell b b$ channel when both $W$ bosons decay leptonically. The $t \bar{t}$ events can also contribute background to the $\ell \nu j j$ channel in the case that one $W$ boson decays leptonically.

\section{Single Top Production}

The top quark in single top events is produced via electroweak interaction either from a s-channel or t-channel process as shown in Fig. 2.14 The top quark decays almost $100 \%$ of the time to a bottom quark and a $W$ boson. To make a contribution to the background 


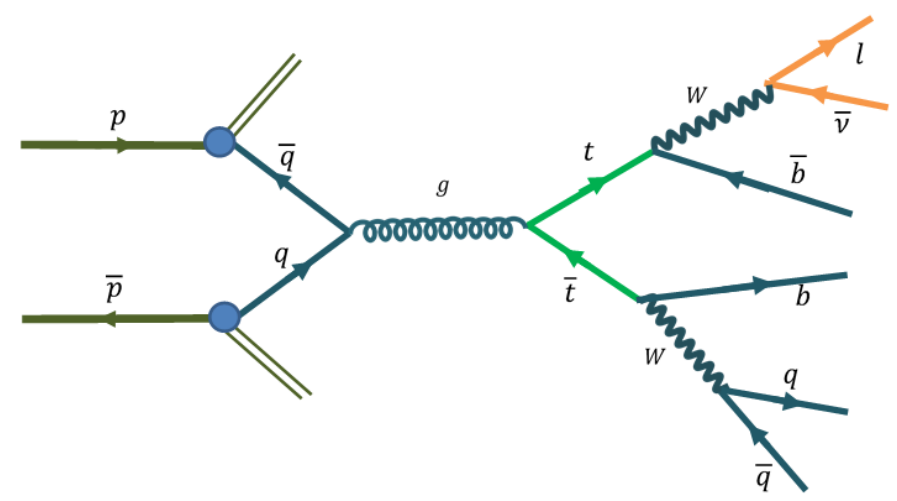

Figure 2.13: Feynman diagram for top pair production

of the $\ell \nu j j$ channel, the $W$ boson in the final states of top quark production must decay leptonically.

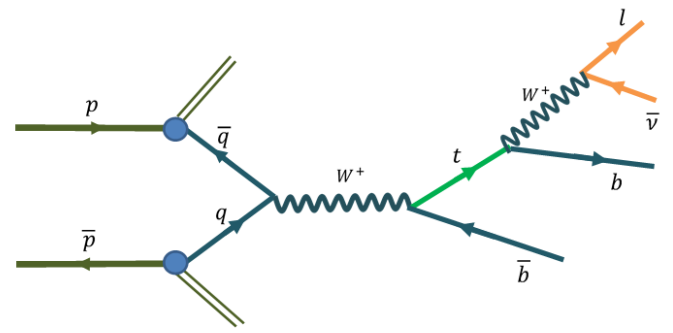

(a) s-channel

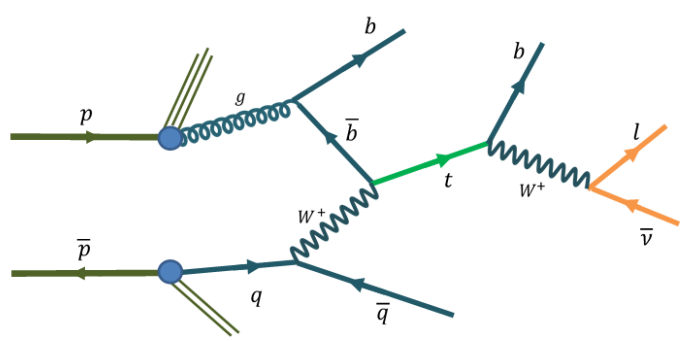

(b) t-channel

Figure 2.14: Feynman diagrams for single top production

\section{Diboson Production}

A pair of weak bosons, $W W, W Z$ or $Z Z$, are produced via diagrams as shown in Fig. 2.15. The diboson events can be background candidates for both $Z H \rightarrow \ell \ell b b$ and $\ell \nu j j$ channels when at least one of the boson decays leptonically.

\section{Multijet}

The only background in $Z H \rightarrow \ell \ell b b$ and $\ell \nu j j$ channels that is not simulated by $\mathrm{MC}$ is the multijet background. The multijet events are produced via the strong interaction and do not contain any isolated charged lepton (Fig. 2.16), but they still can pass the selection criteria and mimic expected signal signatures in the cases that:

- A jet is misidentified as an electron.

- A photon is misidentified as an electron. 


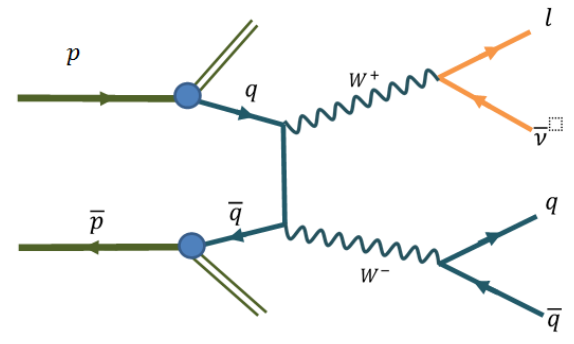

(a) $\boldsymbol{W} \boldsymbol{W}$

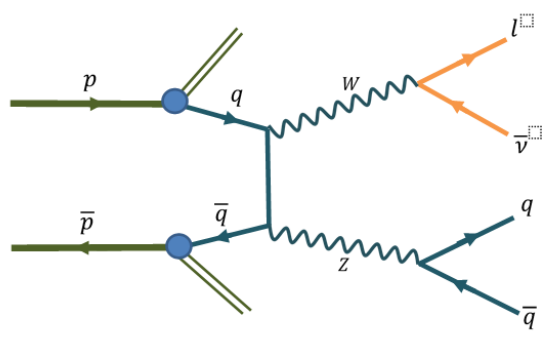

(b) $\boldsymbol{W} \boldsymbol{Z}$

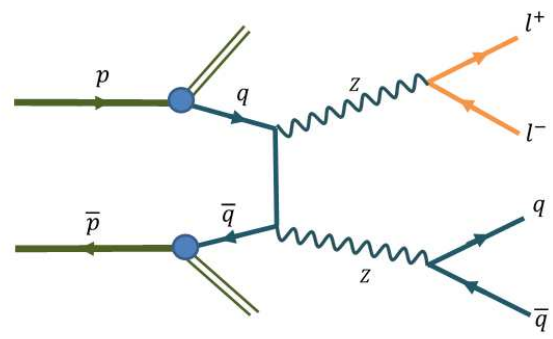

(c) $Z Z$

Figure 2.15: Feynman diagrams for diboson production

- A lepton from decay products of a jet is identified as an isolated lepton.

The description of multijet processes is not precisely modeled by $\mathrm{MC}$, therefore, a datadriven method is used to determine this background. Details about techniques used to derive this background are presented in Sec. 5.5.

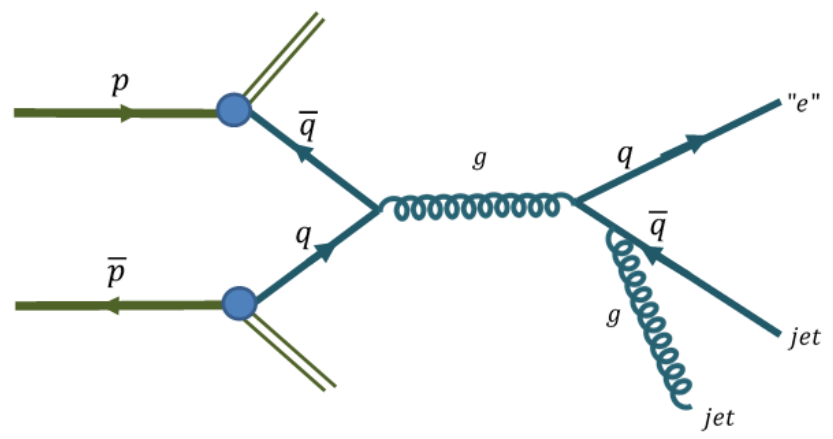

Figure 2.16: One of tree level Feynman diagrams for a multijet process

\subsubsection{Analysis Procedure in $Z H \rightarrow \ell \ell b b$ and $\ell \nu j j$ Channels}

\section{Event selection}

Searching for the Higgs boson in both $Z H \rightarrow \ell \ell b b$ and $\ell \nu j j$ analysis begin with selecting events based on corresponding signal topologies as well as the kinematic differences between 
signal and background. The selection criteria are designed to maximize both the signal acceptance and background suppression in each channel.

\section{Background estimation}

After applying selection criteria, detailed studies of simulated background samples and estimated multijet background are necessary to ensure that data are well described by the SM processes that dominate the selected events. Both shape and normalization of the multijet background are estimated from data. Corrections to background for the discrepancies between data and simulation are derived separately for each channel. These are described in detail in Sec. 5.6.

\section{Tagging for b-quark jets}

The b-tagging technique is applied in both channels to identifying jets originating from b-quarks. It is used to enhance the signal and suppress background in $Z H \rightarrow \ell \ell b b$ channel. Considering the differences in signal compositions of different signal processes, the b-tagging requirements are used in $\ell \nu j j$ channel to divide data into sub-channels (WH $\rightarrow \ell \nu b b$, $H \rightarrow W W \rightarrow \ell \nu$ and $W H \rightarrow W W W \rightarrow \ell \nu j j j j)$ to improve the signal sensitivities.

\section{Multivariate analysis}

Based on the differences in kinematics between signal processes and various background processes with the same final state, a number of physics quantities that have the potential of discriminating signal from background are identified. Studies to evaluate the discriminating power as well as the agreement between data and total simulated background in the distribution of those kinematic variables are carried out in both $Z H \rightarrow \ell \ell b b$ and $\ell \nu j j$ channels. The $Z H \rightarrow \ell \ell b b$ analysis exploits a special technique, namely a "kinematic fit", to improve the discriminating power of important variables. Finally, a list of good variables is selected for each channels.

Multivariate Analysis (MVA) techniques are employed to incorporate discriminating information from all selected variables into a single powerful discriminant to separate signal from background events. A boosted decision tree (BDT) implemented with the TMVA package [47] and a random forest decision tree (RF) [48] implemented in the STATPATTERNRECOGNition (SPR) [49] package are two MVA techniques used in $\ell \nu j j$ analysis. The RF method and Matrix Element method, in which the final signal discriminant is built from calculated probabilities of each observed event being from a signal or background process, are applied in the $Z H \rightarrow \ell \ell b b$ analysis. 


\section{Assessing systematic uncertainties}

The impact of systematic uncertainties on the shape and normalization of the MVA output, the final discriminant distributions, for signal and each background process are assessed. Correlations of each source of systematic uncertainty across all subsamples in each channel are also considered.

\section{Extracting the Higgs boson search results}

The MVA output distributions in each channel along with associated systematic uncertainties are used as inputs for the procedure of setting the upper limits on the SM Higgs boson production cross section multiplied by the corresponding branching fraction in units of the SM prediction. The 95\% C.L. upper limits are determined on the Higgs boson production cross section in the ranges of $90 \leq M_{H} \leq 150 \mathrm{GeV}$ and $90 \leq M_{H} \leq 200 \mathrm{GeV}$ for $Z H \rightarrow \ell \ell b b$ and $\ell \nu j j$ channels, respectively, in steps of $5 \mathrm{GeV}$. The search in the $\ell \nu j j$ channel is also interpreted in the context of fermiophobic and fourth generation models.

The analysis procedure in both $Z H \rightarrow \ell \ell b b$ and $\ell \nu j j$ channeles will be described in this thesis. Research procedures where the author of this thesis mainly contributed will receive more detailed discussion. For the $\ell \nu j j$ channel, the thesis focuses on the process of background estimation, optimizing the MVA for the sub-channel $H \rightarrow W W \rightarrow \mu \nu j j$ and the SM Higg boson search results, as well as interpretation in the fermiophobic and fourth generation models in this sub-channel. For the $Z H \rightarrow \ell \ell b b$ channel, the thesis focuses on the kinematic fit process and Matrix Element approach in discriminating Higgs boson signal from backgrounds. The main Higgs boson search results in $Z H \rightarrow \ell \ell b b$ and $\ell \nu j j$ channel are published separately in Refs. [50] and [10]. The Higgs boson searches in these two channels are also combined with searches in other channels at the DØ and CDF experiments and the combined results published in [12] and [13]. 
3

\section{The Experiment}

\subsection{The Tevatron}

The Fermilab Tevatron is the world's highest energy proton-antiproton $(p \bar{p})$ collider with center-of-mass collision energy $\sqrt{s}=1.96 \mathrm{TeV}$. Providing beams of such high energy requires a complex accelerator chain to produce and accelerate particles. A diagram depicting the Fermilab accelerator chain is shown in Fig. 3.1 [51].

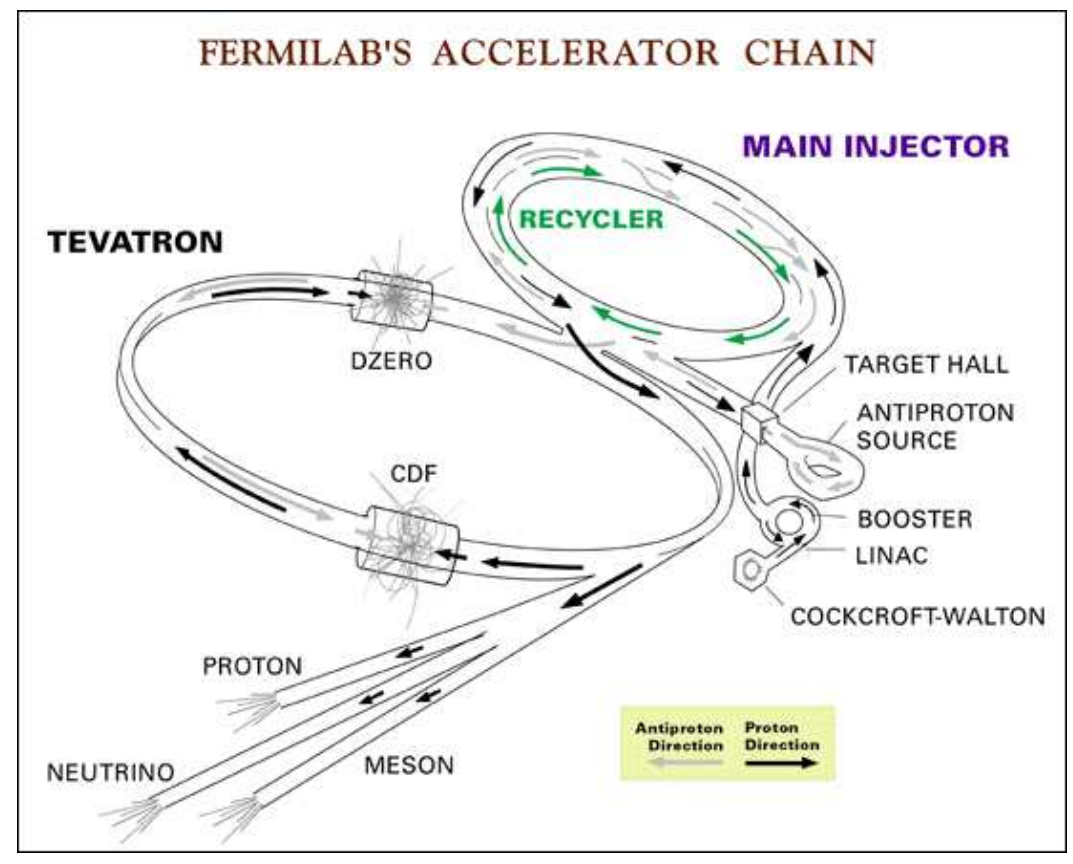

Figure 3.1: Fermilab accelerator chain

At the first step, hydrogen gas is ionized into electrons and protons by electric pulses in an ionization chamber. The released protons strike a negative electrode coated with cesium in the chamber and produce hydrogen ions, $\left(\mathrm{H}^{-}\right)$. The $\mathrm{H}^{-}$ions are focused toward the Cockcroft-Walton pre-accelerator and accelerated to $750 \mathrm{keV}$. They are then steered to the 
linear accelerator (Linac) where they are accelerated to $400 \mathrm{MeV}$ and grouped together to form bunches spaced $5 \mathrm{~ns}$ apart by the oscillating electric fields of radio frequency (RF) cavities.

Leaving the Linac, the $400 \mathrm{MeV} \mathrm{H} H^{-}$ion beam is injected into the Booster, a synchrotron accelerator, through a carbon foil where the two electrons are stripped off from each $\mathrm{H}^{-}$ ion, leaving a beam of bare protons. The magnetic field in the Booster bends the trajectory of the protons making them move along the ring and the energy of protons is boosted from $400 \mathrm{MeV}$ to $8 \mathrm{GeV}$ by $\mathrm{RF}$ cavities. The $8 \mathrm{GeV}$ beam of protons then goes to a transfer line that leads to the Main Injector.

A part of the proton beam injected in the Main Injector is accelerated to $120 \mathrm{GeV}$ for creating antiprotons and the remainder is accelerated to $150 \mathrm{GeV}$ for feeding into the Tevatron. Antiprotons are produced by colliding the $120 \mathrm{GeV}$ proton beam on a fixed nickel target at the rate of about one antiproton created for each $10^{5}$ incident protons on the target with a wide energy spectrum and a large angular spread. The outgoing antiproton beam is focused by magnetic lenses and undergoes a "cooling" process which shrinks the kinetic energy spectrum of the beam to a mean value of $8 \mathrm{GeV}$. The antiprotons are then accumulated and formed into 36 bunches of about $3 \times 10^{10}$ antiprotons before being transfered back into the Main Injector. This $8 \mathrm{GeV}$ antiproton beam is subsequently accelerated to $150 \mathrm{GeV}$ together with 36 bunches of about $3 \times 10^{11}$ protons and injected into the Tevatron.

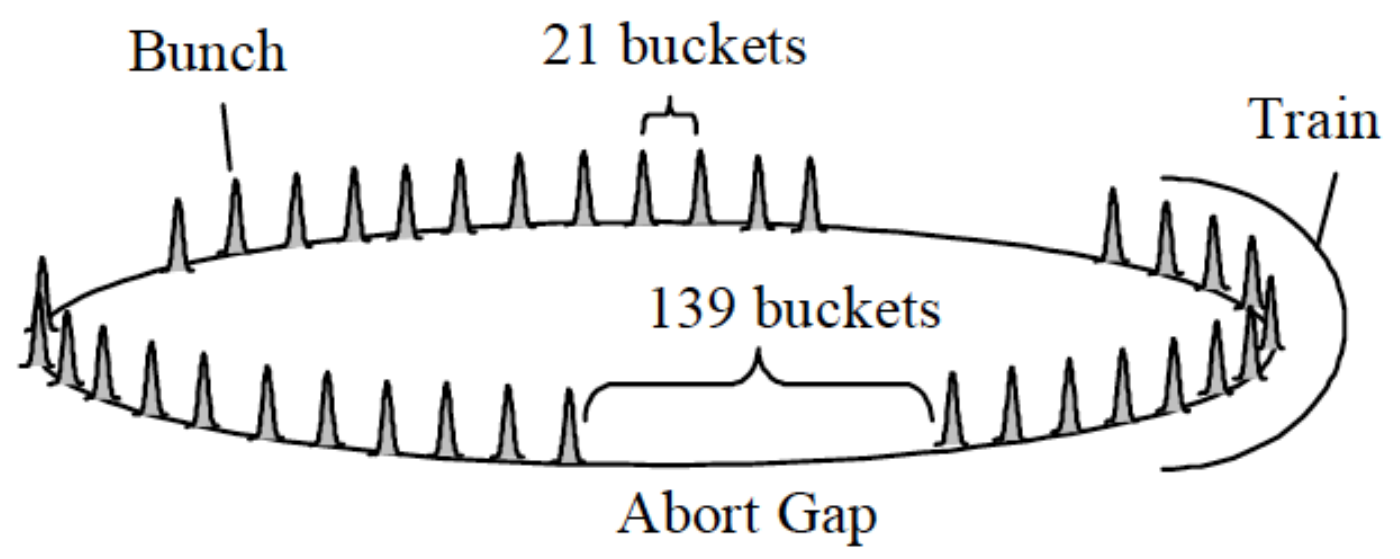

Figure 3.2: The structure of proton and antiproton beams.

The last stage of the Fermilab accelerator chain is the Tevatron, a circular synchroton with a $1 \mathrm{~km}$ radius. It receives the $150 \mathrm{GeV}$ proton and antiproton beams from the Main Injector and accelerates them to $980 \mathrm{GeV}$. The structure of the proton and antiproton beams in the Tevatron is shown in Fig. 3.2, where 36 bunches are grouped in 3 "trains" of 12 bunches [52]. The spacing between bunches within a train is $396 \mathrm{~ns}$ and the 3 trains are separated by $2.64 \mu \mathrm{s}$. The proton and antiproton beams follow separate helical paths throughout most of the beam ring and are shifted to collide at two interaction regions 
located around the centers of the $\mathrm{D} \varnothing$ and CDF detectors. To enhance the interaction rate, quadrupole magnets are used to focus the beams at each collision region.

\subsection{The $\mathrm{D} \varnothing$ Detector}

Two detectors, CDF and DØ, were built around two collision regions of the Tevatron to record and study the outcome of $p \bar{p}$ collisions. The design and performance of the $\mathrm{D} \varnothing$ detector, used to collect the data presented in this thesis, are described in detail in Refs. $[53,54,55,56]$. The major components of this multipurpose detector are shown in Fig. 3.3 and listed from the innermost to the outermost location from the beam pipe as follows:

- Central tracking system

- Preshower detector

- Calorimeter

- Muon system

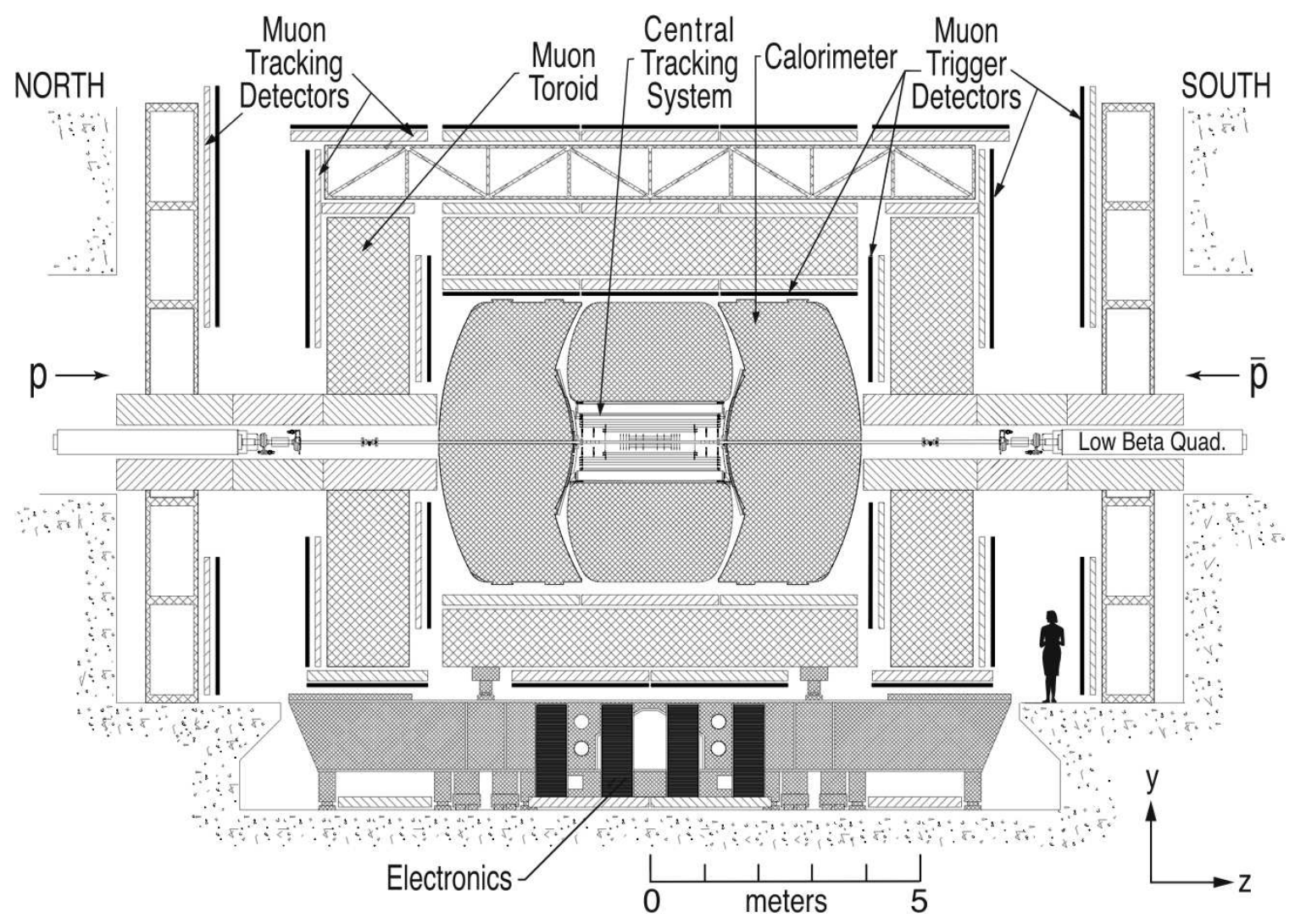

Figure 3.3: Cross sectional side view of the $\mathrm{D} \varnothing$ detector.

The major components of the detector as well as the triggering and data acquisition systems will be briefly described in the next sections. 


\subsubsection{Coordinate System}

In the detector description and data analysis we use a right-handed coordinate system which has z-axis along the direction of proton beam and the y-axis pointing upward as in Fig. 3.3. The $(x, y)$ plane is transverse to the derection of the colliding beams. The angles $\theta$ and $\phi$ are the polar and azimuthal angles, respectively, and the r coordinate denotes the perpendicular distance from the z-axis.

The pseudorapidity, defined as $\eta=-\ln \left(\tan \frac{\theta}{2}\right)$, is commonly used in experimental particle physics as a spatial coordinate. The pseudorapidity of a particle approximates its Lorentz-invariant rapidity, which is defined in terms of energy $(E)$ and longitudinal momentum $\left(p_{z}\right)$ of the particle as $y=\frac{1}{2} \ln \left(\tan \frac{\mathrm{E}+\mathrm{p}_{\mathrm{z}}}{\mathrm{E}-\mathrm{p}_{\mathrm{z}}}\right)$, in the limit that its invariant mass is much less than its energy $m / E \rightarrow 0$. If the $\eta$ quantity is calculated with respect to the center of the detector, it is called detector eta and denoted as $\eta_{\text {det }}$. If the $\eta$ quantity of a particle is calculated based on its reconstructed trajectory from the beam axis, it is called physics eta and denoted as $\eta$.

\subsubsection{Central Tracking System}

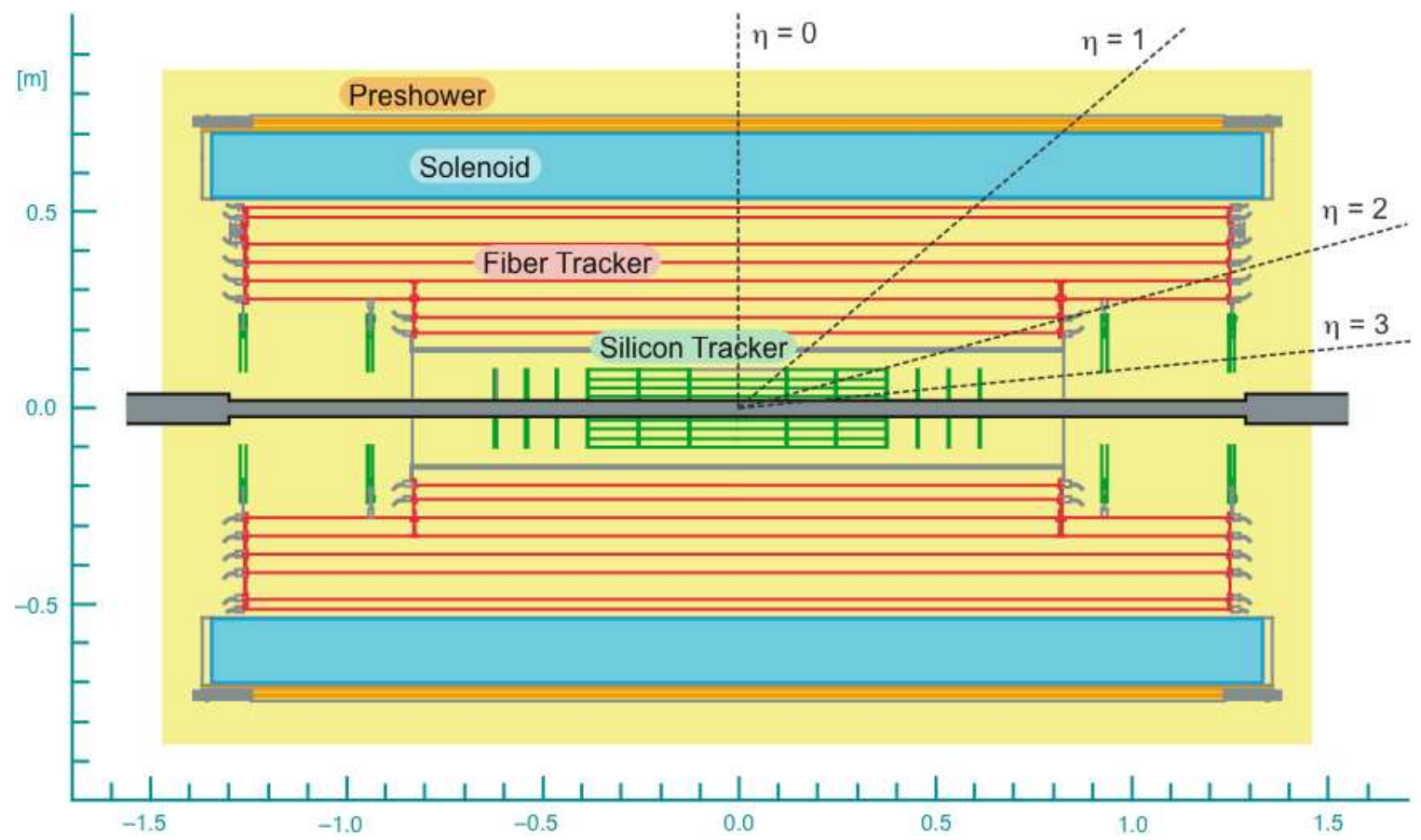

Figure 3.4: Schematic view of the central tracking system.

The central tracking system of DØ detector consists of the Silicon Microstrip Tracker (SMT) and the Central Fiber Tracker (CFT) embedded in a $2 \mathrm{~T}$ magnetic field provided by a superconducting soneloid. The magnetic field bends the path of the charged particles created from the $p \bar{p}$ collisions and the charged particles interact with the SMT and CFT leaving patterns of hit points. The two tracking detectors record the tracks of the charged 
particles with $\left|\eta_{\text {det }}\right|<3$ and locate the $p \bar{p}$ primary interaction vertex with a resolution of about $35 \mu \mathrm{m}$ along the beamline. A schematic view of the central tracking system is shown in Fig. 3.4.

\section{Silicon Microstrip Tracker}

Located closest to the beam pipe, the SMT is designed for tracking charged particles near the interaction points with a high resolution. It is constructed from six silicon barrels, twelve F-disks and four H-disks as shown in Fig. 3.5.

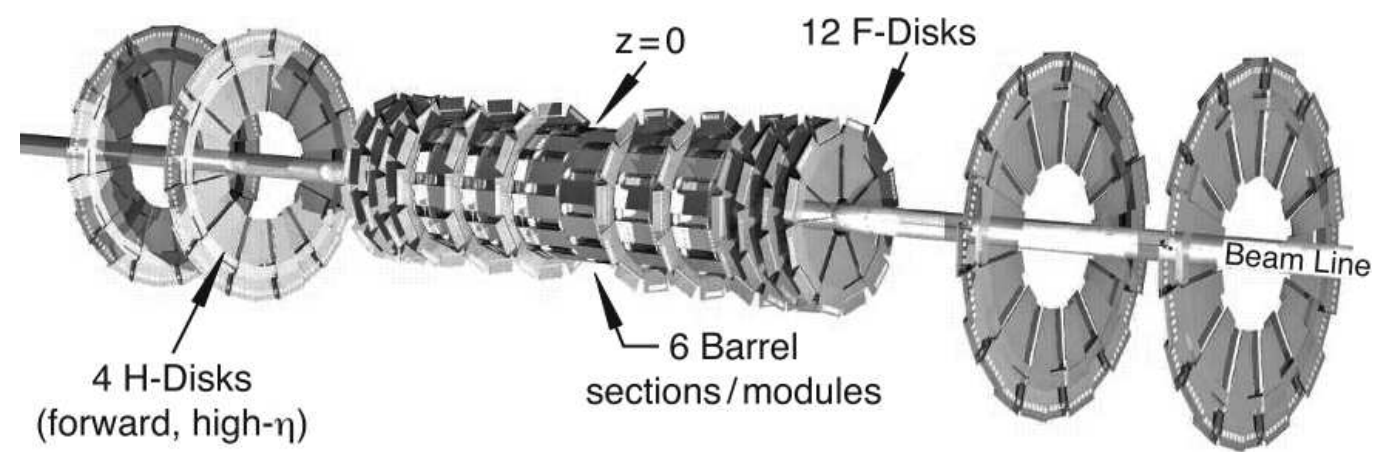

Figure 3.5: Isometric view of the silicon microstrip tracker.

The central region of the SMT comprises six barrels which are arranged along the beam axis with the centers at $|z|=6.2,19.0,31.8 \mathrm{~cm}$. Each barrel s capped at high $|z|$ with an F-disk and has four silicon readout layers, which are set at the distance 2.7, 4.5, 6.6 and $9.4 \mathrm{~cm}$ with respect to the beam pipe. An unit of three F-disks is assembled on each side of the central region. In the far forward region of the SMT, four H-disks are installed at $|z|$ $=100.4$ and $121.0 \mathrm{~cm}$ to provide tracking information at high $\left|\eta_{\text {det }}\right|$.

In 2006, an inner layer called Layer 0 was inserted between the innermost layer of the barrels and the beam pipe to improve tracking resolution and to compensate for radiation damage at the first silicon layer of the barrels [57].

\section{Central Fiber Tracker}

The CFT consists of scintillating fibers arranged in eight concentric cylinders and occupies the radial space from 20 to $50 \mathrm{~cm}$ from the beam axis. Waveguide fibers are coupled to scintillating fibers to transfer the scintillating light produced by an incident charged particle to visible light photon counters (VLPCs) for read out. The CFT provides additional information to determine the momentum of charged particles and reconstruct tracks in region $\left|\eta_{\text {det }}\right| \leq 2.5$. 


\section{Solenoid Magnet}

To improve the detector performance, a superconducting solenoidal magnet was installed in the available space between CFT and the calorimeter: $2.73 \mathrm{~m}$ in length and $1.42 \mathrm{~m}$ in diameter. In order to optimize the momentum resolution and tracking pattern recognition the solenoidal magnet was designed to create a central magnetic field of 2 Tesla. The magnet operates stably at either polarity and the polarity of the magnetic field in the tracking system is frequently reversed to reduce any detector asymmetry effects.

\subsubsection{Preshower Detectors}

Preshower detectors are made of thin layers of scintillator strips interspersed with lead radiators. They are placed in front of the calorimeter and function as tracking detectors as well as calorimeters. They help with electron identification and background rejection during both triggering and offline reconstruction by enhancing the spatial matching between tracks and calorimeter showers. Since particles like electrons and photons may interact with materials in the solenoid and create electromagnetic showers before entering the calorimeters, having the preshower detector in front of calorimeters improves energy reconstruction in the downstream calorimeters.

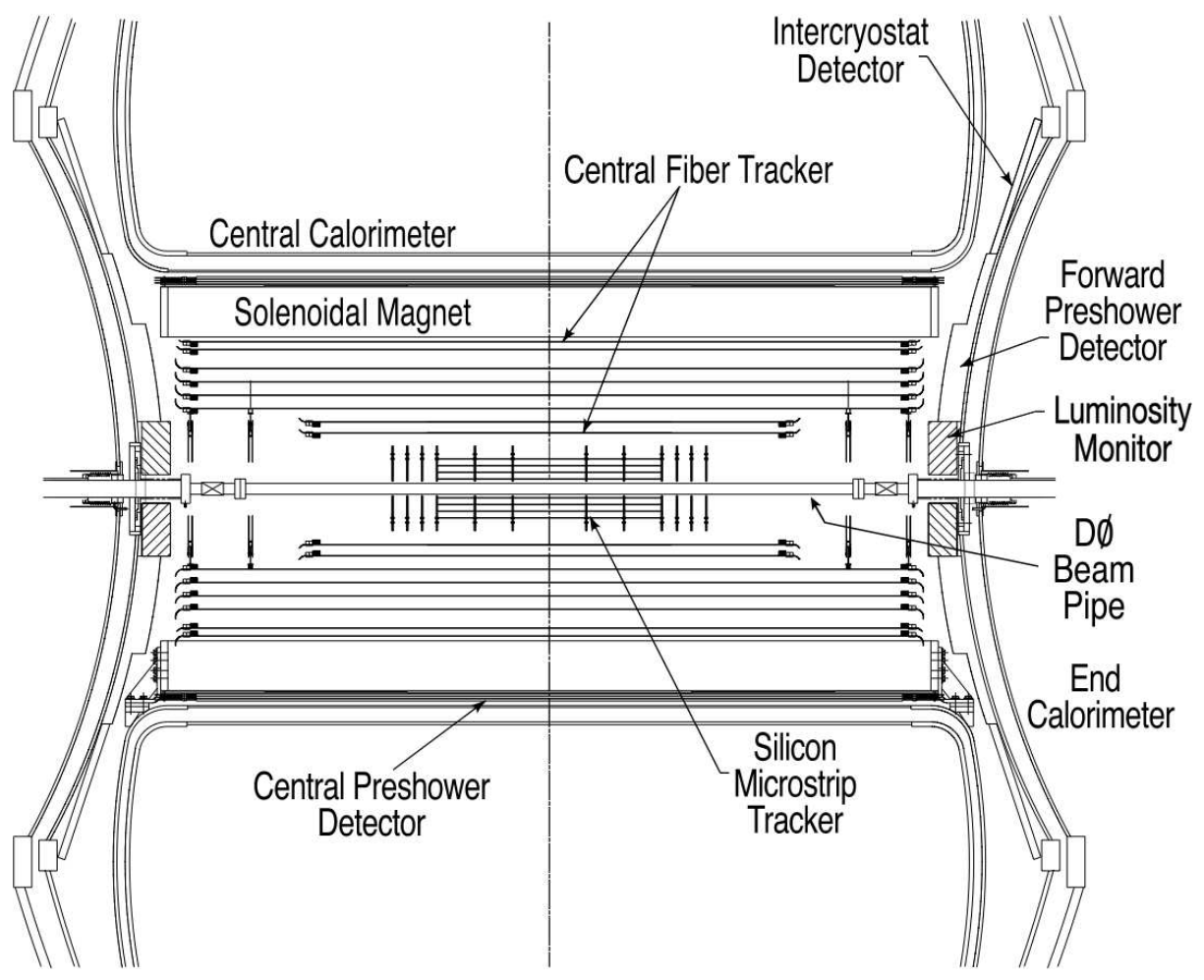

Figure 3.6: Location of major components of the $\mathrm{D} \varnothing$ detector.

The DØ preshower detectors including the central preshower detector (CPS) located between the solenoid and the central calorimeter and two forward preshower detectors (FPS) 
mounted on the end calorimeter are shown in Fig. 3.6. The CPS detector covers the central region $\left|\eta_{\text {det }}\right| \leq 1.3$ and the two FPS detectors cover the forward region $1.5 \leq\left|\eta_{\text {det }}\right| \leq 2.5$.

\subsubsection{Calorimeter System}

The D $\varnothing$ calorimeter system was designed to assist in identification of electrons, photons, and jets, as well as provide energy measurements of those objects, and measure the transverse energy imbalance in events. The system consists of three sampling calorimeters located in separate cryostats, the central calorimeter (CC) and two end calorimeters (EC), and an intercryostat detector (ICD) (see Fig. 3.6). An illustration of the three main calorimeters is shown in Fig. 3.7(a). The central calorimeter covers the region $\left|\eta_{\text {det }}\right|<1$ and the two end calorimeters extend the coverage to $\left|\eta_{\text {det }}\right|<4$. In the transition region $1.1 \leq\left|\eta_{\text {det }}\right| \leq 1.4$ between $\mathrm{CC}$ and EC cryostats, calorimeter coverage is supplemented by the intercryostat detector (see Fig. 3.7(b)).

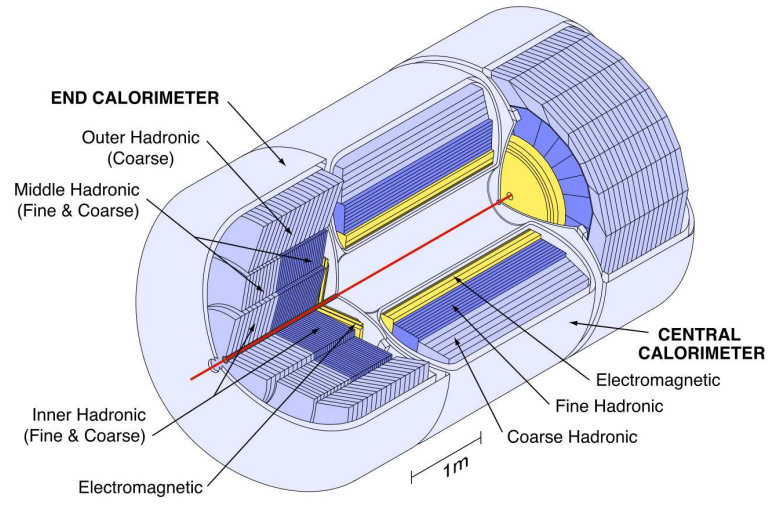

(a) Isometric view of the calorimeter

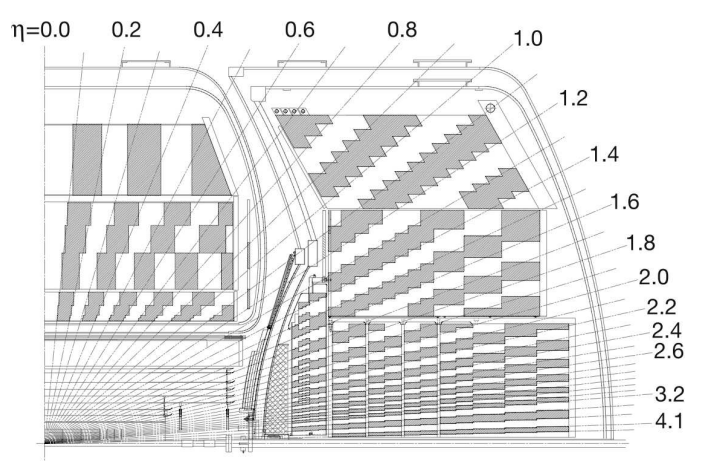

(b) Schematic view of the calorimeter

Figure 3.7: Overview of the DØ calorimeter.

\section{Central Calorimeter and End Calorimeters}

Particles created from $p \bar{p}$ collisions traverse material in calorimeters and lose their energy through different interaction processes resulting from the electromagnetic and strong forces [58]. The types of energy-loss mechanisms that play a role depend on the nature and the energy of the particle. Bremsstralung from electrons and the $e^{+} e^{-}$pair production from photons are the dominant physics processes governing high-energy electromagnetic showers initiated by electrons, positrons or photons. Meanwhile, the hadronic showers initiated by high energy hadrons subject to strong interactions develop based on nuclear interactions. Differences in the primary interactions giving rise to electromagnetic and hadronic showers have crucial consequences in the development of the showers. The longitudinal length and lateral spread of electromagnetic showers are governed by the radiation length $\left(X_{0}\right)$, which mainly depends on the electron density of the material, while the dimensions of hadronic showers depend on the nuclear interaction length $\left(\lambda_{i n t}\right)$, which depends mainly on nuclear 
geometric cross sections [59]. The radiation length and nuclear interaction length for the absorber materials used in the DØ calorimeter are listed in Table 3.1.

\begin{tabular}{||l|c|c||}
\hline \hline Material & $\mathbf{X}_{0}(\mathrm{~cm})$ & $\lambda_{\text {int }}(\mathrm{cm})$ \\
\hline Uranium & 0.32 & 11.03 \\
Copper & 1.45 & 15.32 \\
Iron (steel) & 1.76 & 16.77 \\
\hline \hline
\end{tabular}

Table 3.1: The radiation length $\left(X_{0}\right)$ and nuclear interaction length $\left(\lambda_{\text {int }}\right)$ for the absorber materials used in the $\mathrm{D} \varnothing$ calorimeter.

In order to have good energy resolution for electromagnetic and hadronic showers as well as to improve the performance of particle identification, each of the D $\varnothing$ calorimeters is segmented in two main parts: the electromagnetic (EM) section for measuring electrons and photons, and the hadronic section for measuring particles that pass through the EM section, but range out within material in hadronic sections. The hadronic section in the $\mathrm{CC}$ is subdivided further in the fine hadronic $(\mathrm{FH})$ and the coarse hadronic $(\mathrm{CH})$ sections. Liquid argon is used as the active material for all parts of calorimeters, but different absorber plates are used in different parts of the calorimeters to optimize measurements of different types of particles. Thin plates ( $3 \mathrm{~mm}$ for $\mathrm{CC}$ and $4 \mathrm{~mm}$ for EC) of depleted uranium are used in the EM sections, $6 \mathrm{~mm}$ thick plates made of uranium-niobium (2\%) alloy are used in FH sections, and $46 \mathrm{~mm}$ thick plates made of copper or stainless steel are used in the $\mathrm{CH}$ sections of CC and ECs, respectively. The shower leghth of an incident electron with energy as high as $\sim 300 \mathrm{GeV}$ in the EM section is about $7 X_{0}$, therefore, the D $\varnothing$ electromagnetic calorimeter is a very compact device. The EM section is approcimately $20.0 X_{0}\left(21.4 X_{0}\right)$ thick in the $\mathrm{CC}(\mathrm{EC})$ region. In the $\mathrm{CC}$, the $\mathrm{FH}$ section is about $3.1 \lambda_{\text {int }}$, and in the $\mathrm{EC}$ it ranges from about $3.6 \lambda_{\text {int }}$ to $4.4 \lambda_{\text {int }}$. The $\mathrm{CH}$ calorimeter ranges from $3.2 \lambda_{\text {int }}$ to $6.0 \lambda_{\text {int }}$ in depth.

The calorimeters are constructed from groups of readout cells ganged together as indicated by shaded pattern (Fig. 3.7(b)). Each typical unit cell consists of an absorber plate, a signal board and liquid argon filled in the gaps (see Fig. 3.8). The absorber plates are grounded, while the resistive surfaces of the signal boards are connected to positive voltage $(\sim 2 k V)$ that serve as high voltage electrodes for the gap. The charges liberated from ionizing liquid argon by secondary particles in the showers are collected by the electrodes. Collected charge is then converted into detectable signal.

Several readout pads at approximately the same $\eta_{\text {det }}$ and $\phi$ are linked together to make a readout cell. The transverse size of readout cells is comparable to the transverse size of the showers ( $\sim 1-2 \mathrm{~cm}$ for electromagnetic showers and $\sim 10 \mathrm{~cm}$ for the hadronic showers). The readout cells are then arranged in layers to form towers along lines projected from the center of the interaction region as shown in Fig. 3.7(b). The arrangement and thickness of layers of readout cells are different for the central and end calorimeters. 


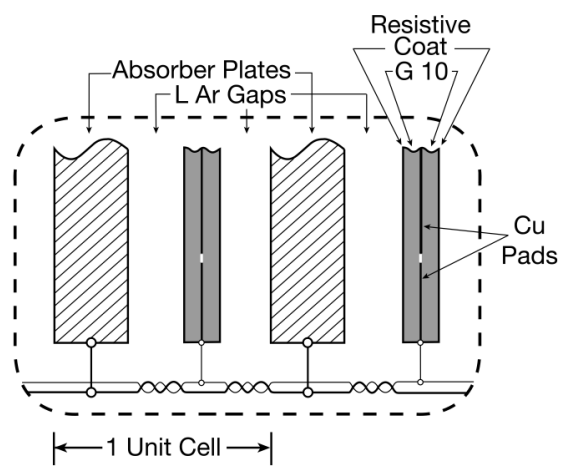

Figure 3.8: Schematic view of a calorimeter cell.

\section{- Central Calorimeters}

The EM section has four layers with thickness of 1.4, 2.0, 6.8 and $9.8 X_{o}$, counting from the innermost part of the CC. There are three layers with thickness of 1.3, 1.0, and $0.76 \lambda_{\text {int }}$ in $\mathrm{FH}$ section, and a single layer with thickness of about $3.2 \lambda_{\text {int }}$ in $\mathrm{CH}$ region.

\section{- End Calorimeters}

The EM section of each EC also have four layers of readout cells with thickness of 1.4, 2.0, 6.8 and $9.8 X_{o}$, in order from innermost layer to the outermost layer. The hadronic section of the EC is separated in three regions: the inner hadronic, the middle hadronic and outer hadronic, as shown in Fig. 3.7(a). Each of the inner and middle hadronic regions is segmented into fine hadronic and coarse hadronic sections, while the outer hadronic region just has the coarse section. The inner hadronic region includes four $1.1 \lambda_{\text {int }}$ thick layers in the FH section and one $4.1 \lambda_{\text {int }}$ thick layer in the $\mathrm{CH}$ section. The middle hadronic region includes four $0.9 \lambda_{\text {int }}$ thick layers in the $\mathrm{FH}$ section and one $4.4 \lambda_{\text {int }}$ thick layer in the $\mathrm{CH}$ section. The $\mathrm{CH}$ section in outer hadronic region has only one layer with the thickness of $6 \lambda_{\text {int }}$.

\section{Intercryostat Detector}

The gaps between CC and EC cryostats along with the presence of substantial unsampled materials used to make cryostat walls degrade significantly the energy resolution in the pseudorapidity region $0.8 \leq\left|\eta_{\text {det }}\right| \leq 1.4$. To address this problem, the intercryostat detector consisting of scintillating tiles is added to sample shower energies between cryostats. The scintillating tiles are attached to the exterior surfaces of the end cryostats covering the $1.1 \leq\left|\eta_{\text {det }}\right| \leq 1.4$ region. Location of the ICD in the D $\varnothing$ detector can be seen in Fig. 3.6.

\subsubsection{Muon System}

As electrons, muons lose their energy through only electromagnetic processes when passing through matter. However, compared to electrons, the cross section for Bremsstrahlung is 
suppressed by a factor of $\left(\frac{m_{\mu}}{m_{e}}\right)^{2} \sim 4 \times 10^{4}$. The dominant energy-loss mechanism for muons at energies below $100 \mathrm{GeV}$ is the ionization process. In fact, muons can penetrate through the $\mathrm{D} \varnothing$ detector without producing a shower in the calorimeter and only deposit about 3 $\mathrm{GeV}$ on average.

The $\mathrm{D} \varnothing$ muon detection system is designed for identification of muons produced in $p \bar{p}$ collisions and determination of their trajectories and momenta. It resides beyond the calorimeter and consists of a toroidal magnet and three layers of muon drift chamber and scintillation detectors. The first layer is before the toroidal magnet and two similar layers of detectors are after the magnet. The muon system is divided into a central section providing the coverage for $\left|\eta_{\text {det }}\right| \leq 1.0$ and the forward section extending the coverage to $\left|\eta_{\text {det }}\right| \leq 2.0$.

\section{Toroidal Magnets}

The toroidal magnets generating a $1.8 \mathrm{~T}$ magnetic field are visible in Fig. 3.3. They allow measurement of the muon momentum independent of the tracking system. This measurement is useful for setting a low- $p_{T}$ threshold in the Level 1 muon trigger (described in Sec. 3.2.7), providing a cleaner matching of muons with central detector tracks, rejecting $\pi / K$ decays, and improving the momentum resolution for high momentum muons.

\section{Muon Drift Chambers}

The central muon system is equipped with proportional drift tubes (PDTs), while the forward muon system uses smaller, faster mini drift tubes (MDTs). The arrangement of three layers composed of PDTs and the MDT is shown in Fig. 3.9. The innermost layer, layer A, located between the calorimeter and the toroidal magnets allows measurement of the muon direction before entering the toroids. The other two layers (B and $\mathrm{C}$ ) reside outside of the magnets and provide measurements of the muon track after being deflected by the magnetic field in the toroids.

For the central muon system, there are four PDT planes in an A layer chamber and three PDT planes in B or $\mathrm{C}$ layer chambers. The PDTs are formed from aluminum cells filled with a mixture of gases ( $84 \%$ argon, $8 \%$ methane, and $8 \% \mathrm{CF}_{4}$ ). Cathode pads are inserted into the top and bottom of each cell and the anode wire is held near the center of the cell. When muons go through cells and ionize the gas, the ions are collected by cathode pads and the freed electrons move toward the anode wires. The electron drift velocity is approximately $10 \mathrm{~cm} / \mu \mathrm{s}$, resulting in a maximum drift time of about $500 \mathrm{~ns}$. The muon coordinate along the wire is measured by a combination of cathode pad signal and timing information from anode wires [53].

For the forward muon system, the MDTs are chosen for the purpose of muon track reconstruction. They are also arranged in three layers A, B and $\mathrm{C}$ of muon chambers as the structure of central muon system. The MDT system is operated with a $\mathrm{CF}_{4}-\mathrm{CH}_{4}(90 \%$ $10 \%)$ gas mixture. The maximum drift time for tracks is between $40-60 \mathrm{~ns}$, depending on 


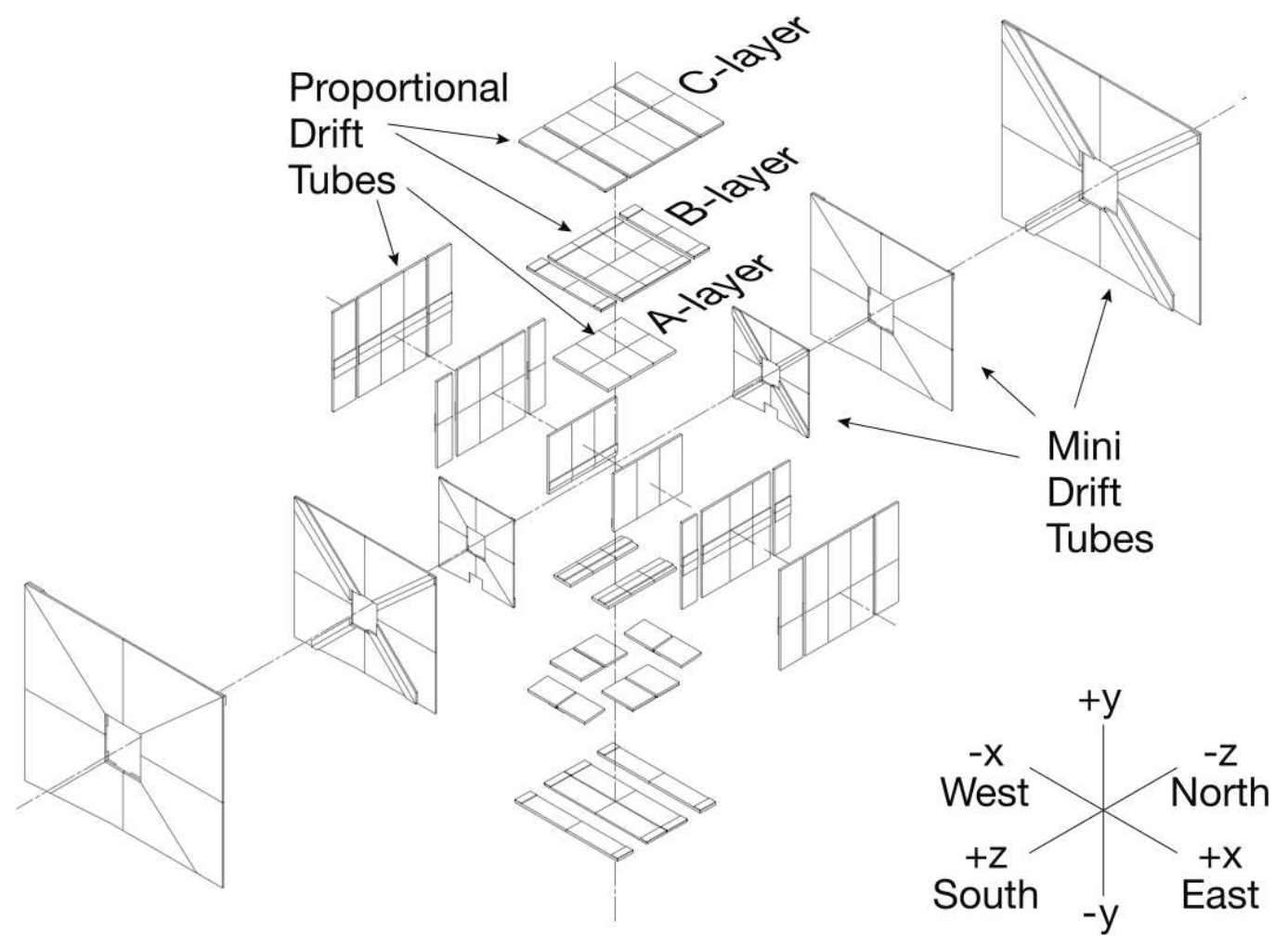

Figure 3.9: Exploded view of the muon wire chamber.

the track direction.

\section{Muon Scintillating Detectors}

Scintillating detectors are installed in both central and forward muon sections for triggering and providing timing information to reject background. The exploded view of muon scintillating detectors is shown in Fig. 3.10.

The cosmic cap and bottom counters are installed on the top, sides and bottom of the outer layer of the central muon PDTs. They provide a fast timing signal to associate a muon in the PDTs with the appropriate bunch crossing and discriminate against cosmic ray muons. The A $\phi$ scintillation counters (see Fig. 3.10) covering the A layer PDTs function as a fast detector for identifying and triggering on muons as well as for rejecting out-of-time background events.

The forward scintillation counters are arranged into three layers and mounted close to the MDT layers. The counters were optimized to provide good time resolution and amplitude uniformity for background rejection, and high muon detection efficiency.

Having to leave space for detector supports and readout electronics, the muon detector coverage in $\left|\eta_{\text {det }}\right|<1.6$ region is limited over the $\phi$ angle, leaving a poorly instrumented region in $-2<\phi<-1.2$ (phi-hole). 


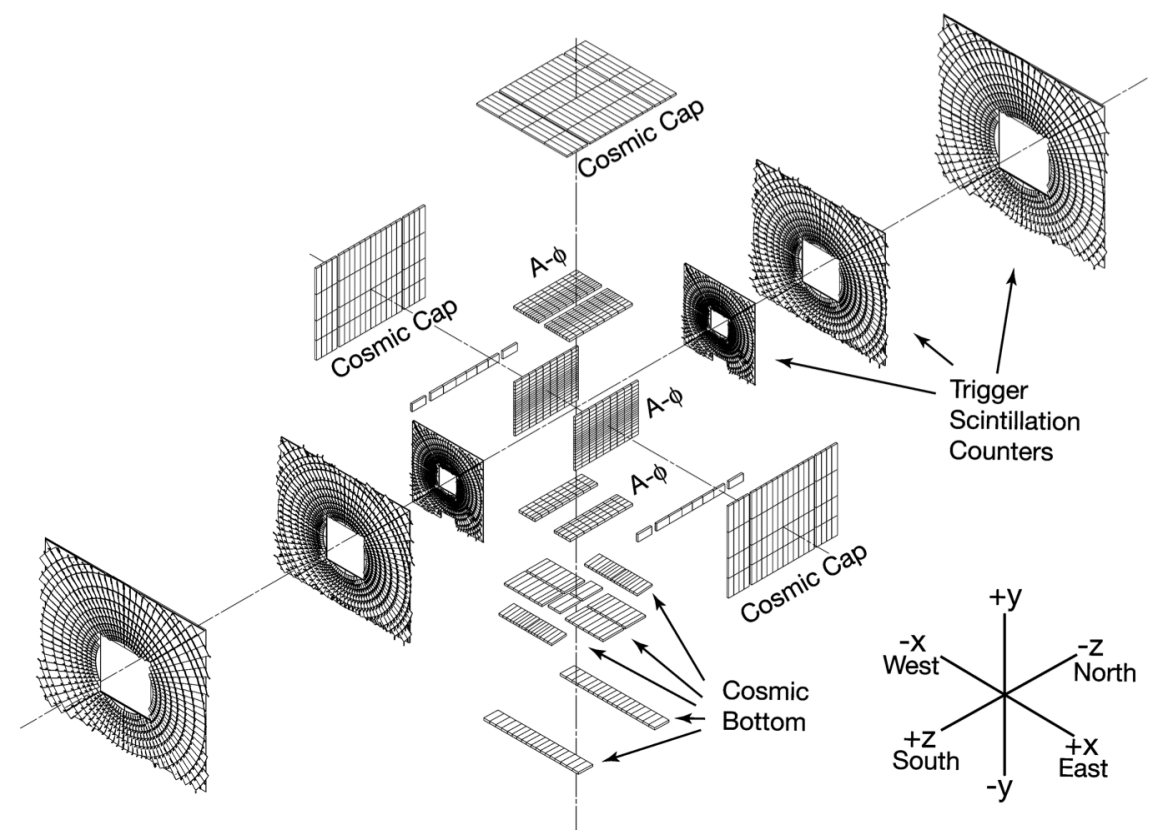

Figure 3.10: Exploded view of the muon scintillating detectors.

\subsubsection{Luminosity Monitor}

The Tevatron luminosity at the $\mathrm{D} \varnothing$ interaction region is determined by detecting inelastic $p \bar{p}$ collisions with the luminosity monitor (LM). The luminosity detector consists of two arrays of 24 scintillating counters located in front of the end calorimeters at $z= \pm 140 \mathrm{~cm}$ and covers the pseudorapidity range $2.7 \leq|\eta| \leq 4.4$ (Fig. 3.11).

The instant luminosity $\mathcal{L}$ is calculated from the average number of inelastic collisions per beam crossing $\bar{N}_{L M}$ measured by the LM:

$$
\mathcal{L}=\frac{f \bar{N}_{L M}}{\sigma_{L M}}
$$

where $\mathrm{f}$ is the $p \bar{p}$ buch crossing frequency and $\sigma_{L M}$ is the effective cross section for $p \bar{p}$ inelastic collisions measured by the LM that takes into account the acceptance and efficiency of the LM detector. The total luminosity accumulated for a period of data-taking is calculated by integrating the instant luminosity over time.

In order to measure luminosity accurately, it is important to distinguish $p \bar{p}$ interactions from the beam halo background. The process of excluding beam halo background is obtained by precise time-of-flight measurements of particles traveling at small angles with respect to the beams and setting a cut on the quantity $z$ calculated from the difference in time of flight:

$$
z=\frac{c}{2}\left(t_{-}-t_{+}\right)
$$

where $t_{-}$and $t_{+}$are the times of flight measured for particles hitting the LM detector placed at $z= \pm 140 \mathrm{~cm}$. If the particles come from beam halo background, $z \approx \pm 140 \mathrm{~cm}$. 
For $p \bar{p}$ interactions, the $z$ value represents the $z$ coordinate of the interaction vertex and is less than $30 \mathrm{~cm}$ for most inelastic collisions. The requirement of $|z|<100 \mathrm{~cm}$ effectively eliminates the beam halo background.

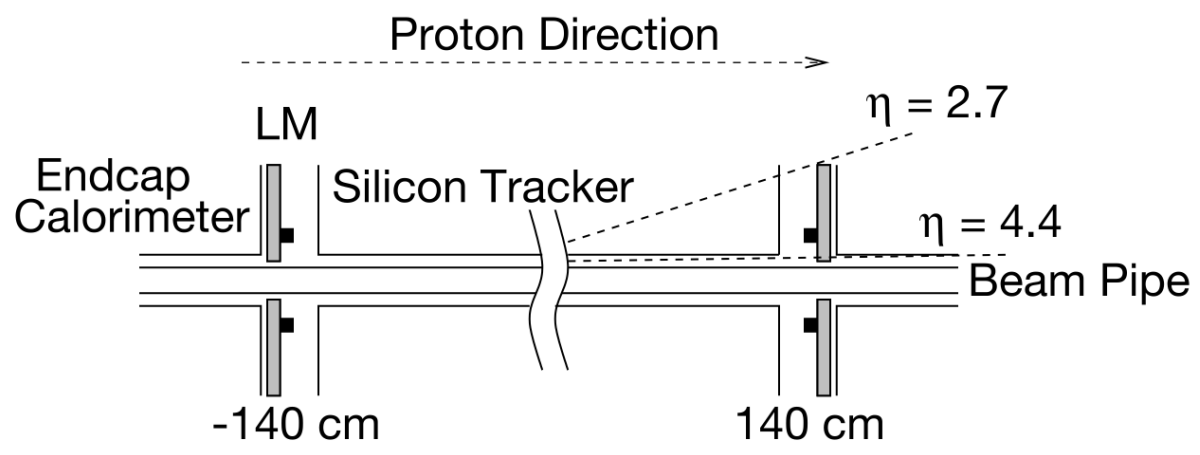

(a) Location of LM detector

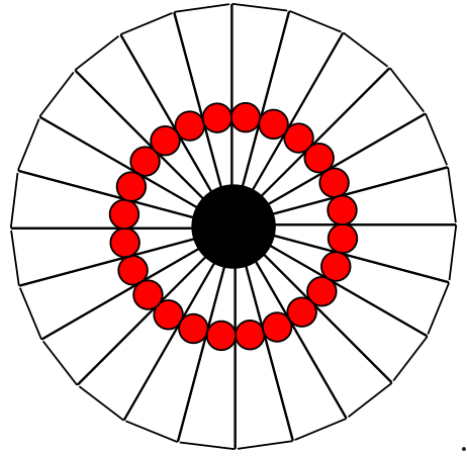

(b) Geometry of LM counters

Figure 3.11: Schematic drawings of the luminosity monitor.

\subsubsection{Trigger and Data Acquisition Systems}

The interaction rate delivered by the Tevatron at the $\mathrm{D} \varnothing$ interaction region has approached $\sim 2 \mathrm{MHz}$. Therefore, it is critical to have efficient trigger and data acquisition systems to select and record only interesting physics and calibration events. The very high selectivity $\left(\sim 1: 10^{5}\right)$ for recorded events at the $\mathrm{D} \varnothing$ detector is achieved through a sequence of three trigger levels of increasing restrictivity and refinement. The recorded data can be then described by the logical "AND" and "OR" of several trigger conditions. A block diagram of the $\mathrm{D} \varnothing$ trigger and data acquisition systems is shown in Fig. 3.12.

\section{Level 1 trigger}

The Level 1 trigger (L1) serves as the first pass in data selection. It uses coarse information from the sub-detector systems to provide a trigger accept rate of about $2 \mathrm{kHz}$. The flowing of the data stream through the L1 trigger system is illustrated in Fig. 3.13. The Level 1 calorimeter trigger (L1CAL) looks for energy deposition pattens with transverse energy 


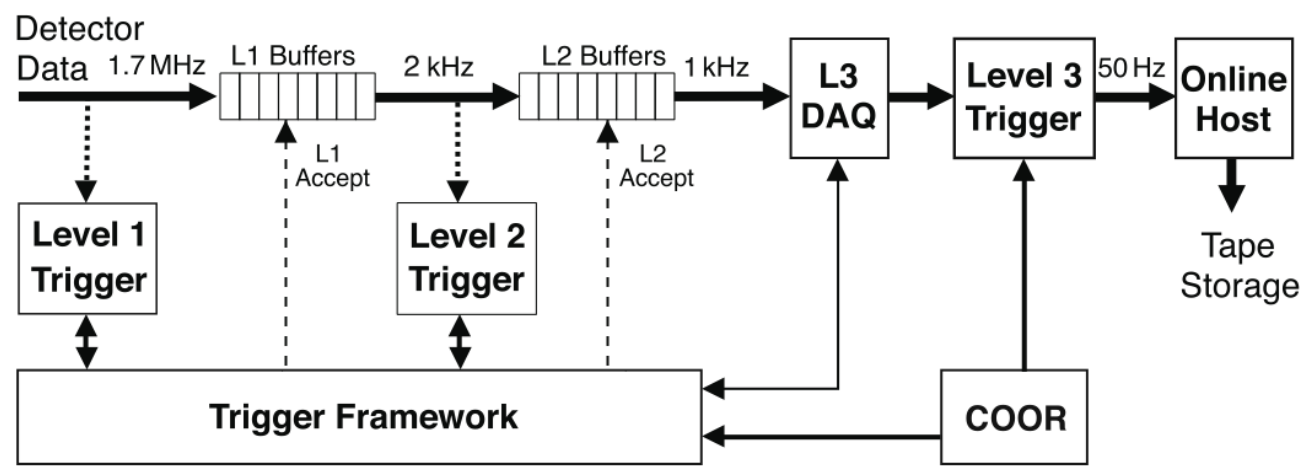

Figure 3.12: Overview of the $\mathrm{D} \varnothing$ trigger and data acquisition systems.

above certain thresholds. The Level 1 central track trigger (L1CTT) looks for hits in the fiber tracker from charged particles which are consistent with predefined models of tracks. The Level 1 muon trigger (L1Muon) uses the hits from muon wire chambers, muon scintillation and the reconstructed tracks obtained in L1CTT to find patterns consistent with muons.

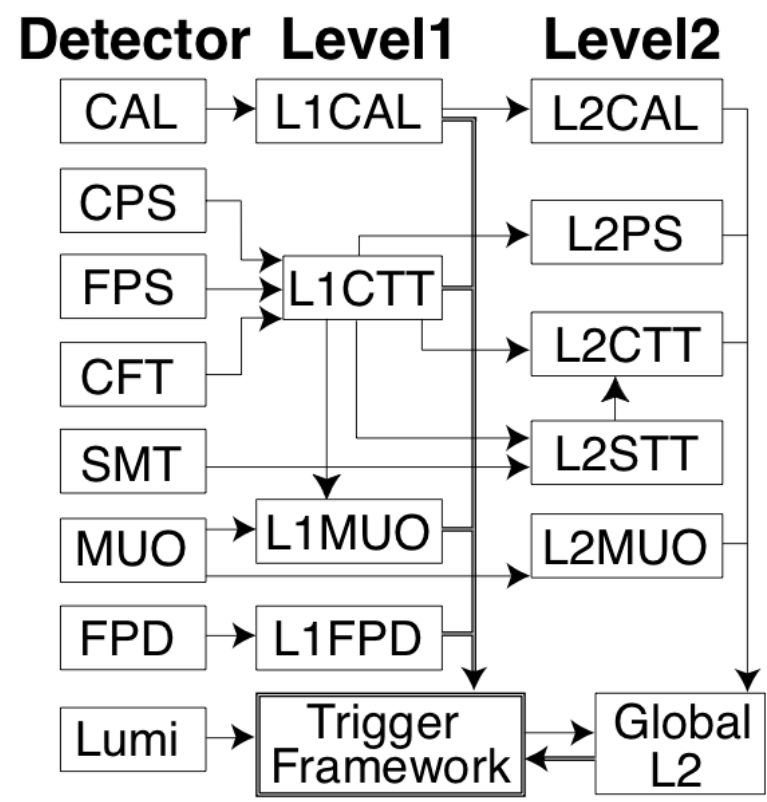

Figure 3.13: The DØ L1 and L2 trigger systems.

The trigger framework (TFW) combines information from each of the specific L1 triggers and makes the decision on whether to select an event for further examination. The TFW is also responsible for applying trigger prescales, which allows removal of events randomly in order to accommodate the recording bandwidth. 


\section{Level 2 trigger}

In the second stage, the Level 2 trigger (L2) uses data collected from L1 triggers and different sub-detectors to construct a global trigger decision based on individual objects as well as object correlations. The L2 system reduces the trigger rate by a factor of about 2 and has an accept rate of approximately $1 \mathrm{kHz}$.

Since the average available time to examine an event is longer than in L1, more complex algorithms can be used and a fast reconstruction of electrons, photons, and jets can be performed in L2. The calorimeter preprocessor system (L2Cal) identifies jets, electrons, photons and calculates the imbalance of transverse energy in an event. The requirements on tracks are tighten by combining tracking information obtained from L1CTT and associated SMT hits to fit the track parameters. At this level, the $p_{T}$ measurements are also refined by using L1CTT output and additional hit and track information than is available at L1. The muon Level 2 trigger (L2Muon) combines the L1Muon output with data from wire chambers and scintillation counter to improve the precision of the track $p_{T}, \eta$ and $\phi$ coordinates and timing information of muon candidates. Global physics objects are created and correlations between objects are examined before trigger decisions are made and events are passed to Level 3 .

\section{Level 3 trigger}

While L1 and L2 trigger systems rely to a large extent on hardware and firmware elements, the Level 3 (L3) trigger is a fully programable software system. The L3 trigger performs a limited reconstruction of events through object-specific software algorithms (such as L3 jet, L3 electron, L3 muon, L3 tracking and L3 missing transverse energy), and reduces the event rate to about $50 \mathrm{~Hz}$. The L3 processing is accomplished by a Linux-farm of about 300 nodes, each with multiple CPU's. When an event is accepted by the L2 trigger, event fragments are sent to the same farm node where they are collated into a complete event available to L3 trigger filter processes. L3 trigger decisions are made based on complete physics objects as well as on correlations between them. Events satisfying L3 trigger requirements are sent to the host cluster to be recorded on tapes in periods of $2-4$ hours and make sequential "runs" of raw data.

\section{Data Acquisition System}

Run control and detector configuration are handled by the central coordination program (COOR) of the data acquisition system. When a new run starts, general trigger and run requests are delivered to COOR which sends out necessary commands to the trigger systems to carry out those requests. After passing L3 filters, event data are collected by the online host system and distributed to logging and monitoring tasks. To take high quality data efficiently, multiple monitor programs are attached to the data acquisition system to quickly spot problems with subdetectors in real time. Event data is examined carefully in the D $\varnothing$ 
detector control room and if there is any problem in the detector performance which appears to effect the data quality, related events are tagged at "bad" by the data quality group and removed from further processing. The monitored event data identified with run number and event number is recorded on tape and used for different analyses by the D $\varnothing$ Collaboration. The data set analyzed in this thesis corresponds to $9.7 \mathrm{fb}^{-1}$ of integrated luminosity and was collected with the D $\varnothing$ detector between March 2001 and September 2011 (Run II). 


\section{Event Reconstruction}

During online data taking, the event data is written on tape from the readout of electrical signals coming from all parts of the detector. Information such as pulse heights, temporal widths and arrival times of those signals in the recorded data must be translated into properties of physics events, so that they can be used to perform all D $\varnothing$ analyses. A sophisticated program called DØ Offline Reconstruction (DØRECO) uses a wide variety of algorithms to reconstruct physics objects from the raw detector data [60]. An overview of the DØRECO program along with techniques and algorithms used for identifying physics objects will be described in the following sections.

\subsection{The DØRECO Program}

The process of reconstructing events in the DØRECO program is structured in three major steps:

\section{- Reconstructing hits and clusters}

At the first step, individual blocks of detector data are decoded and electronic channels are associated with sub-detectors. The information obtained from calorimeter and preshower detectors is then used to reconstruct clusters of calorimeter towers with energy deposits, and the information obtained from the tracking detectors is used to reconstruct the "hits".

\section{- Tracking and vertex finding}

The second step of DØRECO focuses on reconstructing global tracks of particles from the hits in the SMT and CFT detectors. The tracking information is then used in searching for primary vertex (PV) candidates, which indicate the locations of $p \bar{p}$ interaction points. Displaced vertex candidates associated with the decays of long-lived particles are also identified.

- Particle identification

The final step of DØRECO combines the information from each of preceding recon- 
struction steps to produce objects most associated with physics analysis. Candidates of physics objects, such as electrons, photons, muons, jets and missing transverse energy, are created based on clusters of energy, track and vertex objects. This step is essential for a successful translation of the data into physics results.

\subsection{Particle Identification}

\subsubsection{Electron Identification}

\section{Reconstruction and identification criteria}

Being electrically charged and having a relatively low mass, electrons traversing the DØ detector are expected to leave tracks in the central tracking detectors (SMT and CFT) and deposit most of their energy in the EM calorimeter. Therefore, the reconstruction of electron candidates typically proceeds in two stages: a cluster is formed in the EM calorimeter, and subsequent confirmation is sought from the tracking information. Clusters of energy from EM towers are formed with a simple cone algorithm as follow:

- Start with EM towers having $E_{T}>0.5 \mathrm{GeV}$, ordered by decreasing $E_{T}$ and use them as seeds for clustering.

- Centered at each seed tower, all neighboring towers having $E_{T}>0.05 \mathrm{GeV}$ within the cone of radius $\Delta \mathrm{R}=0.3^{1}$ are grouped together. If the total electromagnetic energy of the group of towers within the cone of radius 0.3 satisfies the requirement of being higher than $1 \mathrm{GeV}$, all towers within $\Delta \mathrm{R}=0.4$ are added to form an EM cluster.

- The process is repeated with the highest- $E_{T}$ tower not previously assigned to cluster.

To be identified by the reconstruction program as an electron or photon candidate, the above EM cluster must pass two additional preselection requirements:

- The cluster is required to have more than $90 \%$ of its energy confined in the EM calorimeter. The EM fraction (EMfrac) is defined by the ratio of the energy deposited in the EM calorimeter $\left(E_{E M}\right)$ to the total energy $\left(E_{t o t}\right)$ including the energy deposited hadronic calorimeter, $E M$ frac $=\frac{E_{E M}}{E_{\text {total }}}$. The requirement of having $E M$ frac $>$ 0.9 removes most of candidates caused by hadronic showers, while retains most true electrons and photons.

- Exploiting the characteristic that EM showers are more narrow than the hadronic showers, the isolation of EM clusters is examined through the Iso variable which is defined as the fraction of calorimeter energy in the isolation region bound by the outer

\footnotetext{
${ }^{1}$ The radius of the cone $\Delta \mathrm{R}$ is defined as $\Delta \mathrm{R}=\sqrt{(\Delta \eta)^{2}+(\Delta \Phi)^{2}}$, where $\Delta \eta$ and $\Delta \Phi$ correspond to the sizes of the cone in azimuthal angle and pseudorapidity, respectively.
} 
cone of radius $\Delta \mathrm{R}=0.4$ and the inner $\mathrm{EM}$ cone of radius $\Delta \mathrm{R}=0.2$ to the energy of the EM cluster:

$$
I s o=\frac{E_{t o t}(0.4)-E_{E M}(0.2)}{E_{E M}(0.2)} .
$$

The preselection requirement of having iso $<0.2$ retains more than $98 \%$ of electrons, while significantly reducing the background from jets.

Since photons do not leave tracks as do charged particles, electrons candidates are distinguished from photons by the presence of central tracks associated with EM clusters and pointing to the primary vertexes. A central track is considered to be matched with an EM cluster when it has transverse momentum $p_{T}>1.5 \mathrm{GeV}$ within the window of $\Delta \eta \times \Delta \Phi=0.5 \times 0.5$ around the centroid of the EM cluster. If multiple tracks are found, the selection is made by choosing the track with the best probability for matching the cluster using the spatial distances between track $\eta$ and calorimeter $\eta$ and between track $\phi$ and calorimeter $\phi[61]$.

In order to further reject spurious electrons while retaining high efficiency for identifying true electrons, additional selection criteria based on combined information from the tracking system and calorimeter are applied on top of the preselection requirements. Besides isolation, EM fraction and track-matching variables described above, the following variables are used in our analyses to design more pure selections [62]:

- IsoHC4: representing the track isolation calculated with the total track $p_{T}$ (for tracks with $\left.p_{T}>0.5 \mathrm{GeV}\right)$ in the hollow cone $0.05<\Delta R<0.4$ around the EM cluster:

$$
I s o H C 4=\frac{\sum_{\Delta R>0.05}^{\Delta R<0.4} E_{t r k s}}{E_{E M}(0.2)}
$$

- SigPhi: describing the shower width of the EM cluster at the third layer of the EM calorimeter in the $(\mathrm{r}, \Phi)$ plane.

- HMatrix: characterizing the lateral and longitudinal shower shapes of EM cluster.

- NN: the output of an artificial neutral network trained with variables describing energy deposited and the number of hit cells in the first EM layer, the track isolation and energy deposited in the CPS.

- Lhood8: A likelihood discriminant built from eight quantities characterizing the shapes of both electrons and background. This is a strong variable in discriminating between clusters in the EM calorimeter originating from electrons and those from other processes.

A working point for electron identification (electron ID) is defined by a set of selection cuts applied on above variables. We have applied different requirements for two regions of calorimeters (CC and EC), and several electron ID working points considered in our analyses 
are listed in Table 4.2.1 and Table 4.2.1. The eqn.1 (eqn.2) listed in Table 4.2.1 refering to IsoHC4 $4_{\max }\left(\mathrm{SigPhi}_{\max }\right)$ for the corresponding working points depens on detector $\eta$.

Table 4.1: Electron ID working points in $l \nu j j$ analysis.

\begin{tabular}{|c|c|c|c|c|c|}
\hline \multirow{2}{*}{\multicolumn{2}{|c|}{ Identification variables }} & \multicolumn{2}{|c|}{$\mathrm{CC}$} & \multicolumn{2}{|c|}{ EC } \\
\hline & & Loose & Tight & Loose & Tight \\
\hline EMfrac & $>$ & 0.90 & 0.90 & 0.97 & 0.97 \\
\hline Iso & $<$ & 0.15 & 0.08 & 0.05 & 0.06 \\
\hline IsoHC4 & $<$ & 3.50 & 2.50 & eqn.1a & eqn.1b \\
\hline HMatrix & $<$ & - & 35 & 10.0 & 40.0 \\
\hline NN & $>$ & 0.30 & 0.90 & 0.20 & 1.00 \\
\hline Lhood8 & $>$ & - & 0.20 & - & 0.65 \\
\hline SigPhi & $<$ & - & - & eqn. 2 & eqn. 2 \\
\hline
\end{tabular}

$$
\begin{array}{rr}
\text { eqn.1a } & \text { IsoHC4 }<0.01|| \text { IsoHC4 }<\left(-2.0 \times\left|\eta_{D}\right|+5.0\right) \\
\text { eqn.1b } & \text { IsoHC4 }<0.01|| \text { IsoHC } 4<\left(-2.5 \times\left|\eta_{D}\right|+7.0\right) \\
\text { eqn.2 } & \left|\eta_{D}\right| \leq 2.6: \text { Sigphi }>\left(6.5 \times\left(\left|\eta_{D}\right|-0.82\right)^{-1}-2.8\right) \\
\text { eqn.2 } & \left|\eta_{D}\right|>2.6: \text { Sigphi }>\left(6.5 \times\left(\left|\eta_{D}\right|-1.35\right)^{-1}-2.8\right)
\end{array}
$$

Table 4.2: Electron ID working points in $Z H \rightarrow l l b b$ analysis.

\begin{tabular}{||c|c|c||}
\hline \hline Identification variables & CC & EC \\
\hline EMfrac $>$ & 0.90 & 0.97 \\
Iso $<$ & 0.09 & 0.05 \\
IsoHC4 < & 4.00 & - \\
TrkMatch $>$ & 0.00 & - \\
HMatrix < & - & 10.0 \\
NN $>$ & 0.40 & 0.20 \\
Lhood8 $>$ & - & 0.20 \\
SigPhi < & - & 100 \\
\hline \hline
\end{tabular}

\section{Electron identification efficiency}

The electron identification efficiency (ID efficiency) of data at each working point is determined using the "tag-and-probe" method based on $Z \rightarrow e^{+} e^{-}$candidates having the di-electron mass within the window of $80<M_{e e}<100 \mathrm{GeV}$ [62]. The electron ID efficiency is defined as :

$$
\epsilon=\frac{N_{\text {tag }}}{N_{\text {probe }}}
$$

where $N_{\text {probe }}$ is the number of electron candidates passing the selection cuts relevant to the working point, and $N_{t a g}$ is number of electron candidates passing another set of more stringent selection cuts to reduce the non-Z background and ensure a sample of high purity electron candidates. 
The electron ID efficiency in MC simulation is calculated similarly based on the $Z \rightarrow$ $e^{+} e^{-} \mathrm{MC}$ sample and using the same selection cuts as for data. The number of MC events passing the corresponding set of selection cuts and within the mass window is used to determine the $\mathrm{MC}$ efficiency. The ID efficiency of both data and $\mathrm{MC}$ are parameterized as a function of $\eta$ and $\phi$ components of the electron.

To take into account the imperfection in detector simulation, an efficiency correction is applied to $\mathrm{MC}$ samples by scaling the weight of $\mathrm{MC}$ events by a factor of $\frac{\epsilon_{\text {data }}}{\epsilon_{M C}}$.

\section{Energy calibration correction and resolution}

An absolute calibration of the response of the EM calorimeter is derived based on $Z \rightarrow e^{+} e^{-}$ events considering the boson $\mathrm{Z}$ mass as a calibration point. The measured electron energies consequently are corrected by scaling up so that the mass peak in $Z \rightarrow e^{+} e^{-}$matches the value determined at the LEP collider. The correction is about $0.5 \%$ and $0.1 \%$ in the CC and ECs regions, respectively.

In general, the energy resolution of a calorimeter $\sigma_{E}$ depends on fluctuation in the physical development of the shower, the electronic noise of the readout system and other instrumental effects. For a sampling calorimeter as in $\mathrm{D} \varnothing$ detector, the fractional energy resolution can be expressed as [63]:

$$
\left(\frac{\sigma_{E}}{E}\right)^{2}=\frac{S^{2}}{E}+\frac{N^{2}}{E^{2}}+C^{2}
$$

Where the parameters $S, E$, and $C$ represents different contributing sources to the energy resolution:

- $S$ represents the stochastic term, which is related to fluctuations, and depends on the choice of the absorber, active material and the thickness of sampling layers.

- $N$ is related to electronic noise and depends on the features of the readout circuits. The noise contribution to the energy resolution is dominant at low energy.

- $C$ represents the constant term, which includes contributions from instrumental effects, such as non-uniformity of material thickness, non-uniformity in charge collection, mechanical imperfections, and does not depend on the energy of the particles.

The stochastic term of the electron energy resolution in the $\mathrm{D} \varnothing$ calorimeter is determined by test-beam data, while the noise term is determined by electronics studies and considered to be $0.29 \mathrm{GeV}$ for both $\mathrm{CC}$ and $\mathrm{EC}$ regions [64]. The constant term is dominant in the high energy regime, and is evaluated by using $Z \rightarrow e^{+} e^{-}$events. It is approximately $4 \%$ $(2 \%)$ for CC (ECs) region [64]. 


\subsubsection{Muon Identification}

Muons deposit a very small amount of energy (only about few $\mathrm{GeV}$ ) in the calorimeter and can traverse the entire D $\varnothing$ detector. Therefore, in principle, muons are reconstructed based on the tracking information they leave in the muon detector and the central tracking system.

\section{Muon reconstruction and identification criteria}

The muon reconstruction algorithm employed by the DØ Collaboration can be divided into the following main steps:

- Hit finding

The muon hits (impact positions of muons in the muon system) are determined by using the scintillator counters and the drift time of the wire chambers.

- Segment reconstruction

The 2D track segments in the plane orthogonal to the toroidal magnetic field are built by fitting trajectories to hits in the muon wire chambers. And then identified segments are compared with scintillator hits for timing information on the segment.

- Local muon track finding

Track segments in the A-layer before the toroid and BC-layer after the toroid are matched to form local muon track if they are consistent with the passage of a particle through the magnet. For each compatible pair of segments, a local muon track is reconstructed through a fit taking into account the toroidal magnetic field strength and multiple scattering in the toroids.

- Matching with central tracking

The local muon tracks are matched to the tracks reconstructed in the central tracking system to improve the accuracy of the muon kinematic properties. In the matching process, the tracks are propagated through the calorimeter, taking into account the inhomogeneous magnetic field, energy loss and multiple scattering.

The identification quality of a muon candidate is defined based on the quality of local and central muon tracks as well as the isolation of muon from particles originating from quark fragmentation and other heavy hadron decay products. To reconstruct the candidate $W(\rightarrow \mu \nu)$ or $Z\left(\rightarrow \mu^{+} \mu^{-}\right)$boson in our analyses, the muon candidates in selected events are required to fulfill the following criteria:

- Local muon track with medium quality

To be identified as a local muon with medium quality, the muon candidate must have at least one scintillator hit and two wire hits in the A layer as well as in BC layers. A veto against cosmic muons was imposed by demanding that the scintillator hit times 
in the $\mathrm{A}$ and $\mathrm{BC}$ layers be consistent with the travel time for a particle moving at the speed of light from the primary vertex $\left(\left|t_{A}\right|,\left|t_{B C}\right|<10 \mathrm{~ns}\right)$.

- Central muon track with medium quality

The selected muon candidates are required to have matched tracks in the central tracking system with medium quality. A central track is defined as "medium" if (i) the track distance of closest approach to the beam axis $\mid$ dca $\mid$ is less than $0.04 \mathrm{~cm}$ and the track contains SMT hits or $\mid$ dca $\mid<0.02 \mathrm{~cm}$ for the track without SMT hits, (ii) it has at least two hits in the CFT, and (iii) $\chi^{2} / N_{D O F}<4$ where $\chi^{2}$ is the result of the fit used for reconstruction of the track in the central tracking system and $N_{D O F}$ is the number of degrees of freedom in the fit.

\section{- Loose or tight isolated muon}

Muons coming from leptonic decay of $\mathrm{W}$ or $\mathrm{Z}$ bosons tend to be isolated from jets, while muons originating from decays of heavy hadrons are typically non-isolated due to fragmentation products of hadronic decay. Five discriminating variables were formed to select isolated muons:

- The distance in the $(\eta, \phi)$ space of the muon to the nearest jet with $p_{T}>15 \mathrm{GeV}$, $\Delta R(\mu, j e t)=\sqrt{\Delta \eta(\mu, j e t)^{2}+\Delta \phi(\mu, j e t)^{2}}$.

- The scalar sum of transverse momenta of tracks within the $\Delta R<0.5$ cone around the muon, $I^{\text {trk }}=\Sigma_{(\Delta R<0.5)} p_{T}^{\text {trk }}$.

- The sum of transverse energies of all calorimeter clusters in the hollow cone $0.1<\Delta R<0.4$ around the muon, $I^{\text {cal }}=\Sigma_{(0.1<\Delta R<0.4)} E_{T}^{\text {cluster }}$.

- Two additional isolation variables, $I^{\text {trk }} / p_{T}^{\mu}$ and $I^{\text {cal }} / p_{T}^{\mu}$, were employed to offer stringent rejection of leptons from b-quark and c-quark decays.

The loose isolated muons are required to have $\Delta R(\mu$, jet $)<0.5$. The tight isolated muons are required to have $\Delta R(\mu, j e t)<0.5, I^{\text {trk }} / p_{T}^{\mu}<0.12$ and and $I^{c a l} / p_{T}^{\mu}<0.40$.

In the loose muon samples used to estimate multijet background in our analyses, we require that the muons satisfy the above tracking requirements and the loose isolation criteria. The tight muon events selected to extract the Higgs boson signal in our analyses are required to contain muons satisfying the same tracking requirements as for the loose muon sample and the tight isolation criteria.

\section{Muon identification efficiency and momentum resolution}

The performance of the $\mathrm{D} \varnothing$ detector in identifying and reconstructing muons is quantitatively assessed in terms of muon identification efficiency and momentum resolution [65]. In the region $|\eta|<2$, the efficiency of muon system reconstruction of muon candidates used in 
our analyses ranges from $75 \%$ to $90 \%$. Central tracks matched to these muons are reconstructed with average efficiency of $90.5 \%$. The isolation criteria reject multijet background with efficiencies ranging from $87 \%$ to $92 \%$ depending on quality requirements. The momentum of a muon candidate is taken to be the momentum measured in the central tracking system and the momentum resolution of typically $10 \%$ for $p_{T}=40 \mathrm{GeV}$.

\subsubsection{Jet Identification}

\section{Jet development}

Jets result from the fragmentation of quarks and gluons generated in the hard scattering process of $p \bar{p}$ collisions. The development of jets in the detector can be separated into three sequential stages:

- Quarks and leptons are produced from the hard scattering process, and then these partons can eventually radiate additional partons and form "parton jets".

- Stable particles produced through hadronization of quarks and gluons, excluding undetected muons and neutrinos, are clustered to form "particle jets".

- The spray of produced hadrons interact inside the calorimeter where the "calorimeter jet" is defined and its energy is measured.

A sketch of the evolution from a hard-scatter parton to a jet in the calorimeter is shown in Fig. 4.1.

\section{Jet reconstruction}

Jets are reconstructed using the Run II cone algorithm [66], which is an iterative cone algorithm used to build jets from energy deposits in the calorimeter. The first step of jet reconstruction is defining the jet seeds by clustering pseudoprojective towers in $\eta \times$ $\phi$ calorimeter cells. Each cell is treated as a massless object and has an associated 4momentum computed using the direction defined by the primary vertex and the center of the cell and assuming $E=|p|$. The 4-momentum of a pseudoprojective tower is formed by combining the 4-momentum of calorimeter cells using the E-scheme:

$$
P^{\text {tower }}=\left(E^{\text {tower }}, \vec{P}\right)=\sum_{i=\text { cells in tower }}\left(E_{i}, \overrightarrow{p_{i}}\right)
$$

The calorimeter towers are ordered in decreasing transverse momentum and used to form preclusters within a cone of radius 0.3 in the $(\eta, \phi)$ plane, starting with the tower having the highest $p_{T}$ and descending the list until no towers above the minimum threshold $p_{T}>500 \mathrm{MeV}$ remain. Preclusters with $p_{T}>1 \mathrm{GeV}$ are used as seeds for the jet clustering algorithm. 


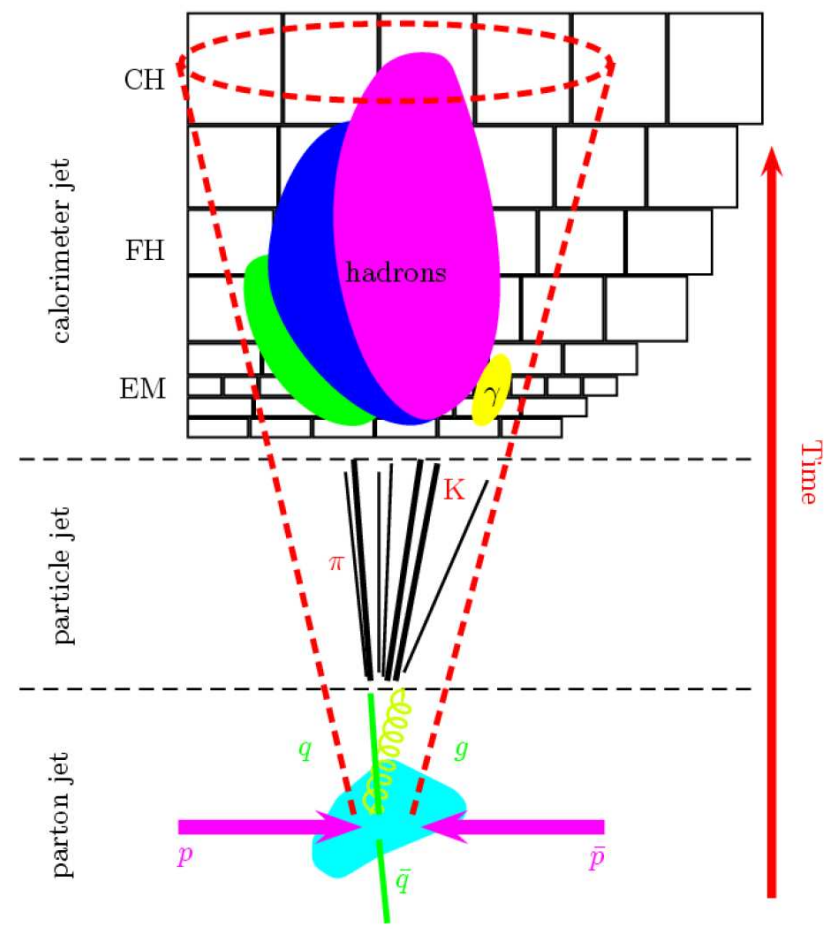

Figure 4.1: The evolution from a hard-scatter parton to a jet in the calorimeter.

The seeds are then used as center points and all calorimeter towers around the seeds within the cone of radius $R_{\text {cone }}=0.5\left(\Delta R=\sqrt{(\Delta y)^{2}+(\Delta \phi)^{2}}<R_{\text {cone }}\right)$ are combined to form the proto-jets. The 4-momemtum of a proto-jet is the sum of the 4-momenta of all included calorimeter towers. When the direction of the 4-momentum of the protojet does not coincise with the cone axis, the process is repeated using the direction of the proto-jet 4-momentum as the new center point for the cone until a stable solution is found. To reduce the sensitivity to soft radiation, mid-points between pars of two protojets are also used additional seeds, if the distance between two proto-jets is in the range of $R_{\text {cone }} \leq \Delta R \leq 2 R_{\text {cone. }}$. Any proto-jets having the transverse momentum below a minimum threshold $p_{T \text { (protojet) }}<3 \mathrm{GeV}$ are discarded.

The obtained proto-jets may contain partially overlapping or identical jet candidates. To avoid double counting tower energies the proto-jets are sorted in order of decreasing $p_{T}$ and processed through a split-or-merge procedure to remove overlaps. Two proto-jets are merged into one jet if they have an overlap region containing more than $50 \%$ of the transverse momentum of the lower $p_{T}$ proto-jet. Otherwise, two proto-jets are split into two jets and each cell in the overlap region is assigned to the nearest jet in the $(y, \phi)$ plane. The jet 4-momentum is recomputed after the split-or-merge process and the jets with $p_{T}<8 \mathrm{GeV}$ are discarded. 


\section{Jet identification}

To eliminate jet candidates not originating from outgoing partons of the hard scattering process, a set of jet identification criteria are imposed:

- Requirement on the electromagnetic fraction: $0.05<$ EM frac $<0.95$.

The energy fraction in the EM layers is required to be less than $95 \%$ in order to reject electron- and photon-like objects. The requirement of having electromagnetic fraction higher than $5 \%$ has to be fulfilled to reject fake jets coming from noises in the hadronic calorimeter.

- Requirement on the coarse hadronic fraction: CHfrac $<0.40$.

The energy fraction in the outermost layer of the calorimeter, the coarse hadronic layer, is required to be less than $40 \%$. This requirement is designed to further remove jets that are formed predominantly out of the noise in the hadronic calorimeter.

\section{- L1 trigger confirmation}

A Level 1 trigger confirmation is obtained by requiring the jet energy measured by the independent electrical readout of L1 to be larger than $50 \%$ of the jet energy, excluding the energy deposited in the coarse hadronic calorimeter cells $(\mathrm{CH}$ is not included at L1). This removes jets formed out of noise, such as coherent noise in the precision readout electronics.

- Vertex confirmation

To ensure a selection of jets originating from the hard scattering process, the criteria of a jet to be vertex confirmed are imposed. A vertex confirmed jet must be associated with at least two tracks orginating from the primary vertex. These tracks must have $p_{T}>0.5 \mathrm{GeV}$, at least one hit in the SMT, distance of closest approach to the beam line in the transverse plane $D C A_{x y}<0.5 \mathrm{~cm}$, distance of closest approach to the beam line in the z direction $D C A_{z}<1 \mathrm{~cm}$, and $\Delta z($ vertex, track $)<2.0 \mathrm{~cm}$.

\section{Jet energy scale}

The jet energy scale (JES) corrects on average the energy of jets measured in the calorimeter to the corresponding particle jets. This correction accounts for the differences in the observable jets from the particle level due to detector and jet algorithm effects [67]. Correcting the jet energy measured in the $\mathrm{D} \varnothing$ detector $E_{\text {meas }}$ to the particle level $E_{\text {particle }}$ is performed in three stages: (i) correction of offset energy $(O)$, (ii) correction of jet energy due to the calorimeter response $(R)$, (iii) correction for showering effects $(S)$. These corrections can be expressed by the formula:

$$
E_{\text {particle }}=\frac{E_{\text {meas }}-O}{R \cdot S} .
$$

The main effects considered when calibrating jet energy will be briefly discussed before evaluating the final JES. Additional corrections for small biases in the method are described 
elsewhere [67].

\section{- Offset energy correction (O)}

The offset energy consists of the energy in the jet that is not related to the primary $p \bar{p}$ interaction. It includes a component corresponding to energy from multiple $p \bar{p}$ interactions during a bunch crossing (MI) and a component originating from electronic noise, calorimeter noise from uranium decay and the pile-up energy from other bunch crossing (NP). The value of the MI offset depends linearly on the number of additional interactions, which is characterized by the number of reconstructed $p \bar{p}$ interaction vertices $\left(N_{P V}\right)$ in a given event. Fig. 4.2 shows the offset correction as a function of jet pseudorapidity in the detector for different numbers of reconstructed primary vertices. The uncertainties on the offset corrections are of the order of $1 \%$ of the overall energy correction at low jet $p_{T}$ and are negligible for jet $p_{T}$ above $\sim 100 \mathrm{GeV}$.

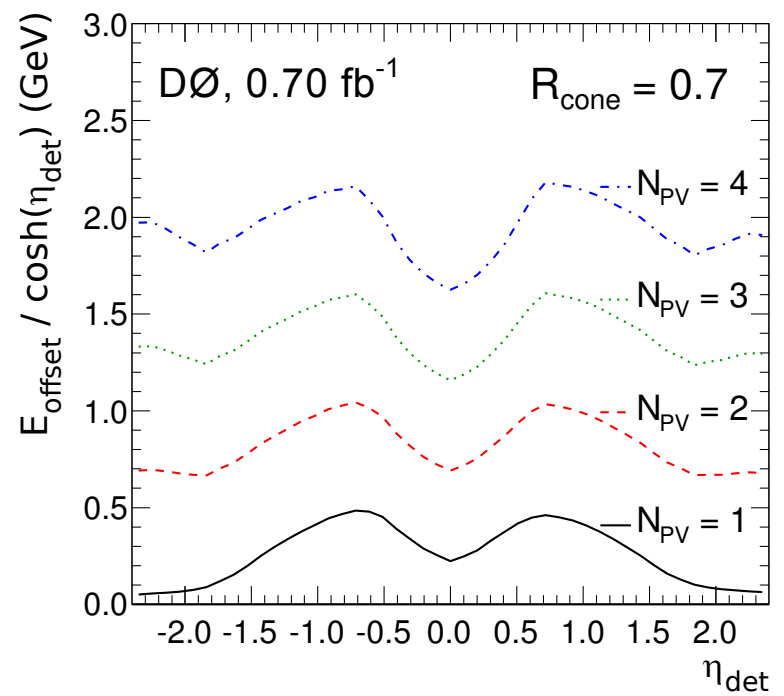

Figure 4.2: Offset corrections for different primary vertex multiplicities.

\section{- Calorimeter response correction ( $R$ )}

The calorimeter response $R$ is the average fraction of the energy measured in the calorimeter for the particles inside the jet cone. It is factorized into two parts $R=R_{C C}(E) \times F_{\eta}(\eta, E)$, where the $R_{C C}$ term is an absolute response correction determined from the $\mathrm{CC}$ region and the relative response correction term $F_{\eta}$ normalizes the response of the calorimeter as a function of jet pseudorapidity.

\section{Absolute response correction}

The response for jet energies in the $\mathrm{CC}$ region is derived by using the $p_{T}$ balance between the $\gamma$ and the jet in the $\gamma+$ jet events. To have a clean $\gamma+$ jet data sample, only photons with tight selection criteria in the $\mathrm{CC}$ region are used. The jet is required to be back-to-back to the photon $\left(\Delta \phi_{(\gamma, j e t)}>2.9\right)$ and no additional jets are allowed in selected events. At the particle level, the transverse momenta of the photon and 
the hadronic recoil system are balanced each other:

$$
\vec{P}_{T, \gamma}+\vec{P}_{T, j e t}=\overrightarrow{0}
$$

Due to non linearities in the energy response of the calorimeter, the measured jet $P_{T, j e t}$ becomes unbalanced with the photon $P_{T, \gamma}$ causing a non-zero $\mathrm{E}_{\mathrm{T}}$ :

$$
R_{j e t} \cdot \vec{P}_{T, j e t}+R_{\gamma} \cdot \vec{P}_{T, \gamma}=-\vec{E}_{T}
$$

where $R^{j e t}$ and $R_{\gamma}$ are electromagnetic and hadronic energy responses of calorimeter, respectively. Using the corrected photon energy scale $\left(R_{\gamma}=1.0\right)$ and projecting Eq. 4.9 onto the direction of photon transverse momentum, the jet response is defined as:

$$
R_{\text {jet }}=1+\frac{{\overrightarrow{E_{T}}} \cdot \vec{P}_{T, \gamma}}{\left|\vec{P}_{T, \gamma}\right|^{2}} .
$$

The jet energy response in the $\mathrm{CC}$ region shown in Fig. 4.3 is measured as a function of:

$$
E^{\prime}=p_{T, \gamma} \cdot \cosh \left(\eta_{j e t}\right),
$$

which is highly correlated with the particle level jet energy and has finer resolution than the measured jet energy. The dependence of jet response to $E^{\prime}$ is later mapped to the measured jet energy to determine the correction to be applied to the specific jet algorithm. The dominant sources of uncertainty on jet $p_{T}$ response come from photon identification, photon energy scale, fragmentation and PDF, and the total uncertainty is about $\sim 1 \%$.

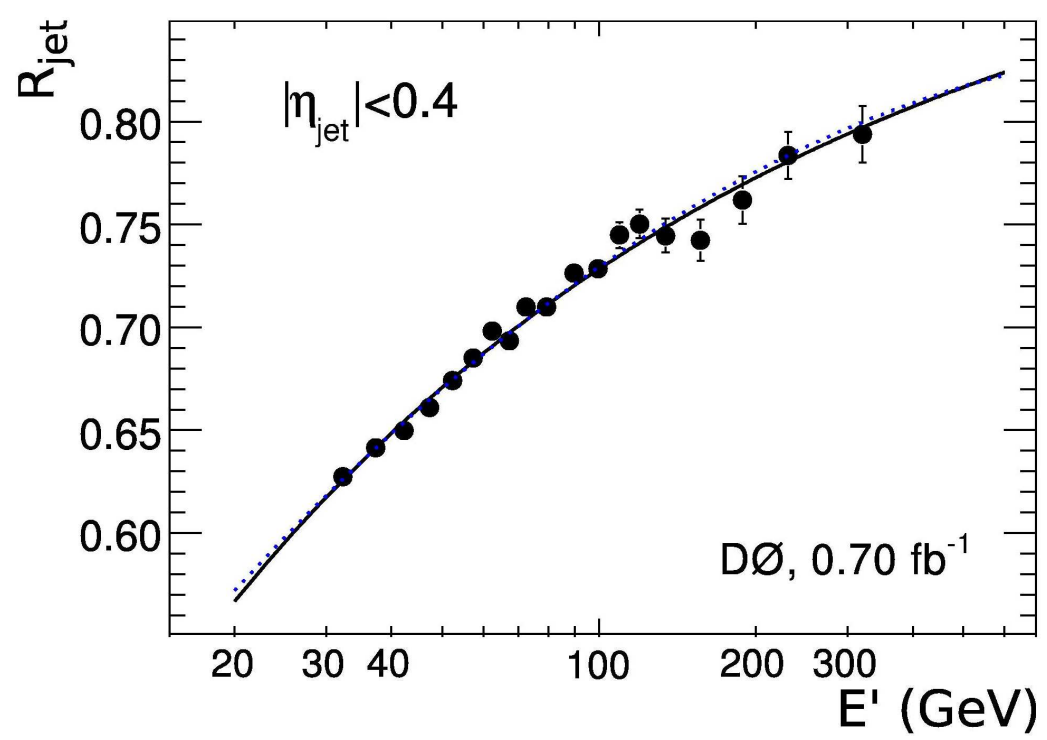

Figure 4.3: Fits of the jet energy response in the $\mathrm{CC}$ region using $\mathrm{MC}$ points (solid line) and data (dotted line) [67]. 


\section{Relative response correction}

The relative response correction, $F_{\eta}$, calibrates forward jets with respect to central jets. It normalizes the response at forward pseudorapidities to that measured in the $\mathrm{CC}$ region $\left(R_{C C}\right)$ and is defined as:

$$
F_{\eta}(E, \eta) \equiv \frac{R(E, \eta)}{R_{C C}(E)}
$$

where $R(E, \eta)$ is the response of detector for a jet of energy $\mathrm{E}$, located at detector pseudorapidity $\eta$. Deriving $F_{\eta}$ is based on the $p_{T}$ balance in two samples, $\gamma+$ jet and dijet. For the dijet sample, one of the jets is required to be in the $\mathrm{CC}$ and the response measurement is binned in E' using the $p_{T}$ of the central jet after correcting for offset and central calorimeter response. The $\eta$-dependence of the relative response is parameterized as a quadratic-logarithmic function of E':

$$
F_{\eta}\left(E^{\prime}, \eta\right)=\frac{p_{0}(\eta)+p_{1}(\eta) \ln \left(E^{\prime}\right)+p_{2}(\eta) \ln ^{2}\left(E^{\prime}\right)}{R_{C C}\left(E^{\prime}\right)},
$$

where $p_{i}$ are fitted as functions of $\eta$. Fig. 4.4 shows the $\eta$ dependence correction in the region of $0.8<\eta<0.9$ for the $\gamma+$ jet and dijet samples. The difference in $F_{\eta}$ fits observed in Fig. 4.4 is due to the different amount of quark and gluon jets in the $\gamma+$ jet and dijet samples. The gluon initiated jets have lower response than quark initiated jets since they have on average higher particle multiplicity with softer particles.

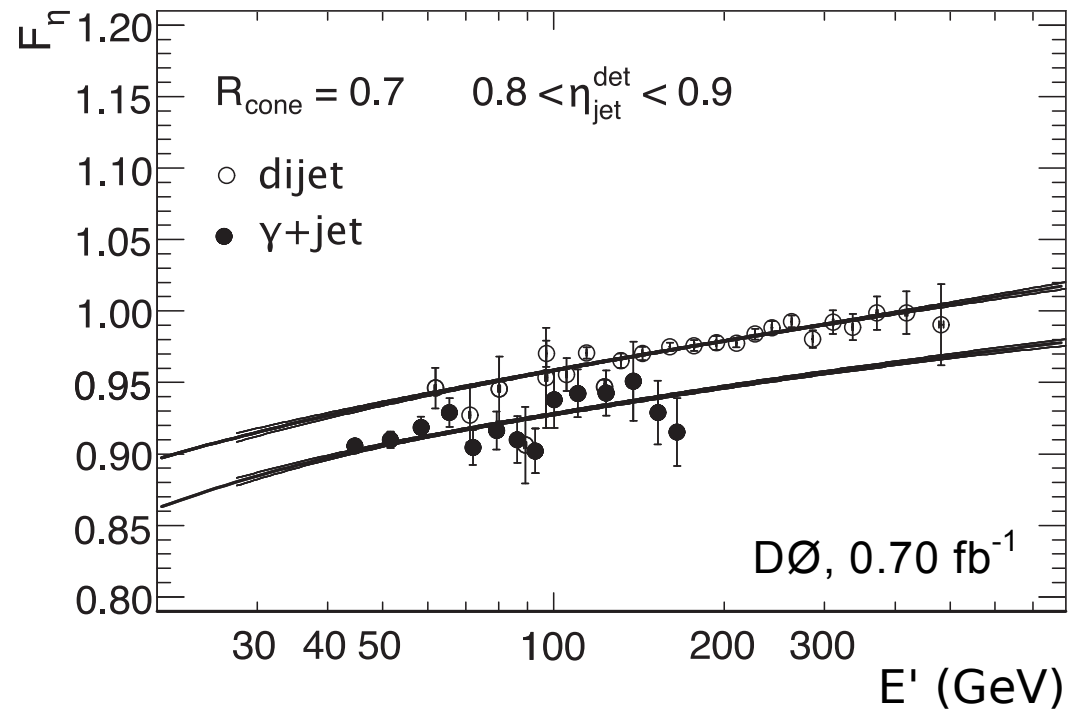

Figure 4.4: Fits of $F_{\eta}$ in $\gamma+$ jet and dijet as a function of E' [67].

\section{- Showering correction (S)}

Showering effects contribute to the mismeasurement of jet energy due to the net flow of energy in and out of the jet cone caused by detector effects, such as the magnetic 
field, scattering from passive material, and shower development in the calorimeter. The determination of the showering correction is based on comparison of jet energy profiles between MC and data using a sample of back-to-back $\gamma+$ jet events. Energy density profiles of jets in $\mathrm{MC}$ are generated as a function of the distance $\Delta R=\sqrt{\left(y_{\text {particle }}-y_{\text {jet }}\right)^{2}+\left(\phi_{\text {particle }}-\phi_{\text {jet }}\right)^{2}}$ between the particle and jet axis. The calculated energy profile in $\mathrm{MC}$ is fitted to measured energy profile after the offset correction has been applied. An example of energy profiles as function of $\Delta R$ for MC and data in one $\eta, p_{T}$ bin is given in Fig. 4.5. It shows the good agreement between measured energy in data and the sum of energy contributions originating from inside and outside the particle jet. The showering correction in each $\left(\eta, p_{T}\right)$ bin is estimated

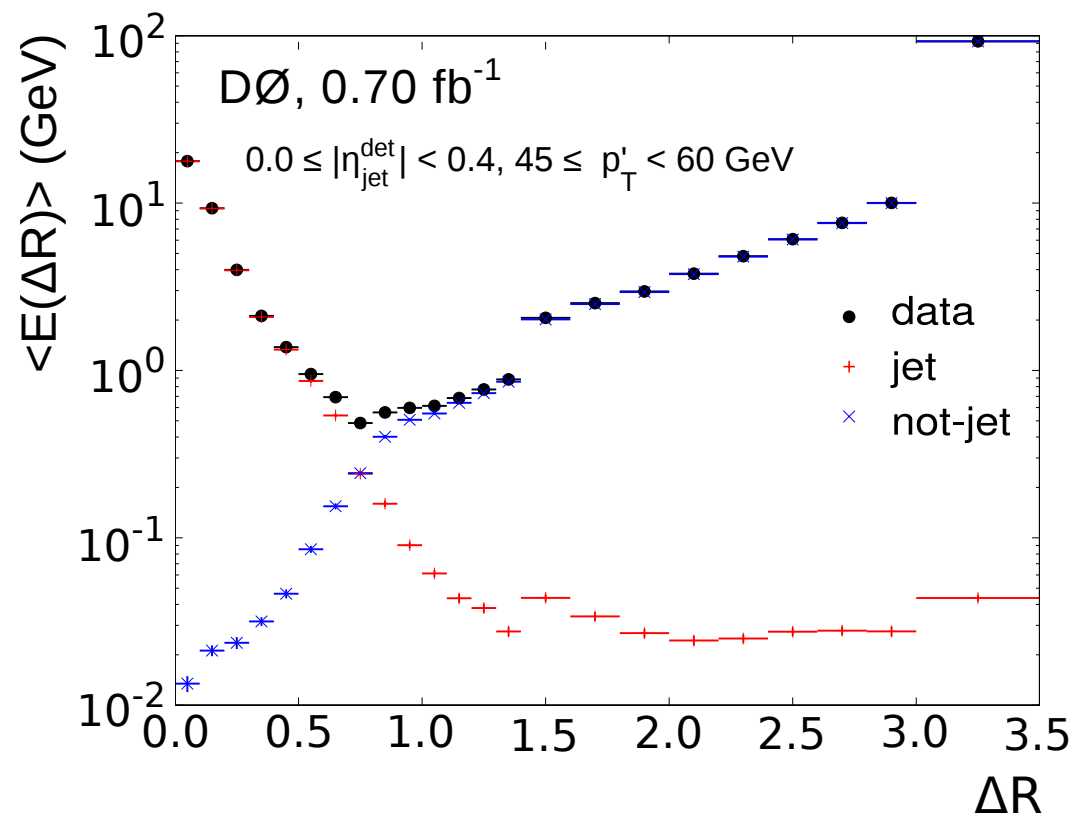

Figure 4.5: Showering profiles as function of distance from jet axis $\Delta R$ for data and MC [67].

as:

$$
S=\frac{\Sigma_{\Delta R=0}^{R_{\text {cone }}} E_{\text {in }}+\Sigma_{\Delta R=0}^{R_{\text {cone }}} E_{\text {out }}}{\Sigma_{\Delta R=0}^{R_{\infty}} E_{\text {in }}},
$$

where $\Sigma_{\Delta R=0}^{R_{\text {cone }}} E_{\text {in }}$ is the energy of the particle jet deposited inside the jet cone, $\Sigma_{\Delta R=0}^{R_{\text {cone }}} E_{\text {out }}$ is the energy deposited inside the jet cone from particles outside the jet, and $\Sigma_{\Delta R=0}^{R} E_{i n}$ is the total enegy of particle jet obtained using the fit-weighted templates.

The systematic uncertainties on the showering correction are mainly driven by the quality of the template fit, the physics modeling of jet fragmentation and the purity of the $\gamma+$ jet sample. They are less than $1 \%$ of the overall correction factor at $p_{T}<50 \mathrm{GeV}$. 


\section{Shifting, smearing and removing in simulated jets}

In order to correct simulated jets for the differences with data in terms of energy scale, resolution and reconstruction efficiency, the JSSR procedure which allows simulated jets to be smeared, shifted and possibly removed was developed [68]. In the JSSR procedure, the corrections of simulated jets were derived using the $\gamma+$ jet and $Z+$ jet samples where $\gamma / Z$ and jet in each selected events are required to be back-to-back in azimuth to avoid large effects from QCD radiation. Determination of these corrections relies on the measurement of the transverse momentum imbalance in selected $\gamma+$ jet and $Z+$ jet events:

$$
\Delta S=\frac{p_{T}^{j e t}-p_{T}^{\gamma / Z}}{p_{T}^{\gamma / Z}},
$$

which was binned in $p_{T}^{\gamma / Z}$ separately in different $\eta$ region. An example of the $\Delta S$ distribution for data and $\mathrm{MC}$ are given in Fig. 4.6. The $\Delta S$ distribution in each $p_{T}, \eta$ bin is fitted
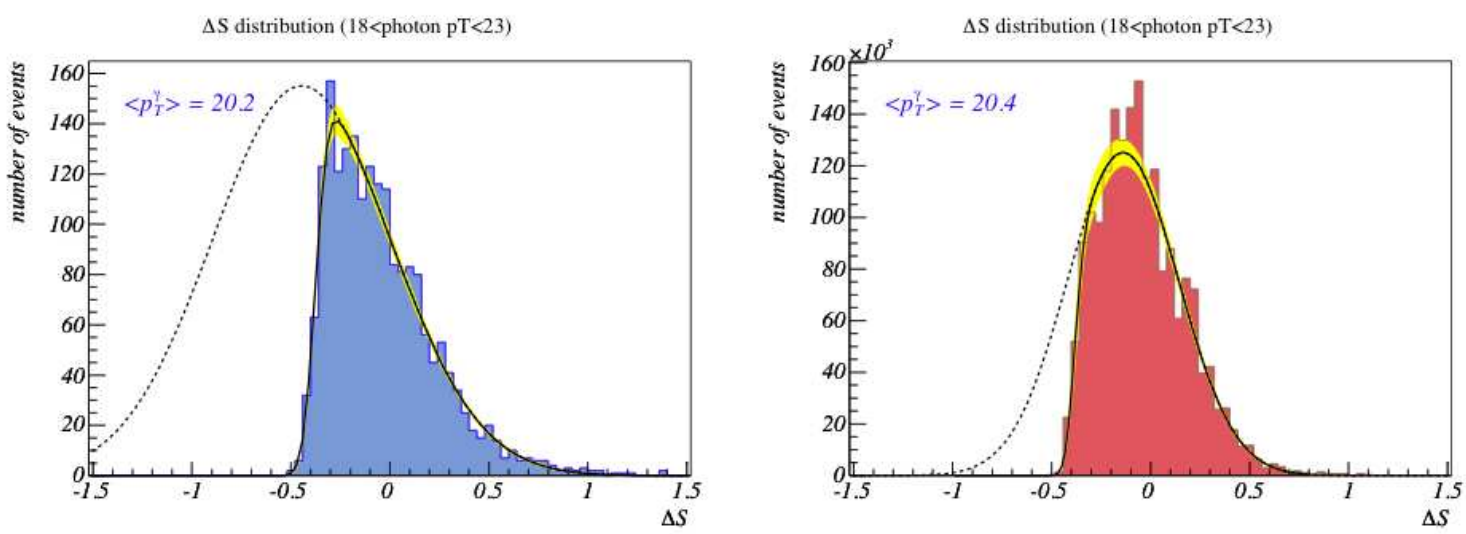

Figure 4.6: $\Delta S$ distributions for data (left) and Monte Carlo (right). The full curves show the fits using a fitted function composed of a Gaussian and an error function. The yellow bands denote statistical error. The dashed curves correspond to the extrapolation of the gaussians to the region effected by the jet reconstructed threshold.

with a function composed of a Gaussian and an error function to count for resolution and efficiency effects $[68,69]$. The full curves in Fig. 4.6 show the fit results and the dashed curves correspond to the extrapolation of the gaussians to the region effected by the jet reconstructed threshold. These fits provide simultaneously information on:

- the jet energy resolution via the width of the Gaussian.

- the jet energy scale via the central value of the Gausian

- the jet reconstruction and identification efficiency via the error function.

\section{Smearing}


The jet transverse energy resolution in data and $\mathrm{MC}$ can be deduced from the corresponding Gaussian width of the $\Delta S$ distribution:

$$
\sigma_{\Delta S}^{\text {Gauss }}=\frac{\sigma_{p_{T}}}{p_{T}^{\gamma / Z}}
$$

The smearing process corrects for jet resolution differences in data and MC by scaling the transverse momentum of simulated jets with a smearing factor which is randomly drawn from a new Gaussian having width computed as:

$$
\sigma_{\text {smearing }}=\sqrt{\sigma_{\Delta S}^{2}(\text { Data })-\sigma_{\Delta S}^{2}(M C)},
$$

where $\sigma_{\Delta S}^{2}($ Data $)$ and $\sigma_{\Delta S}^{2}(M C)$ are the gaussian width of the $\Delta S$ distribution measured in data and Monte Carlo, respectively. Fig. 4.7 shows the width of smearing factor Gaussian for simulated jets in CC region as a function of $p_{T}^{Z}$.

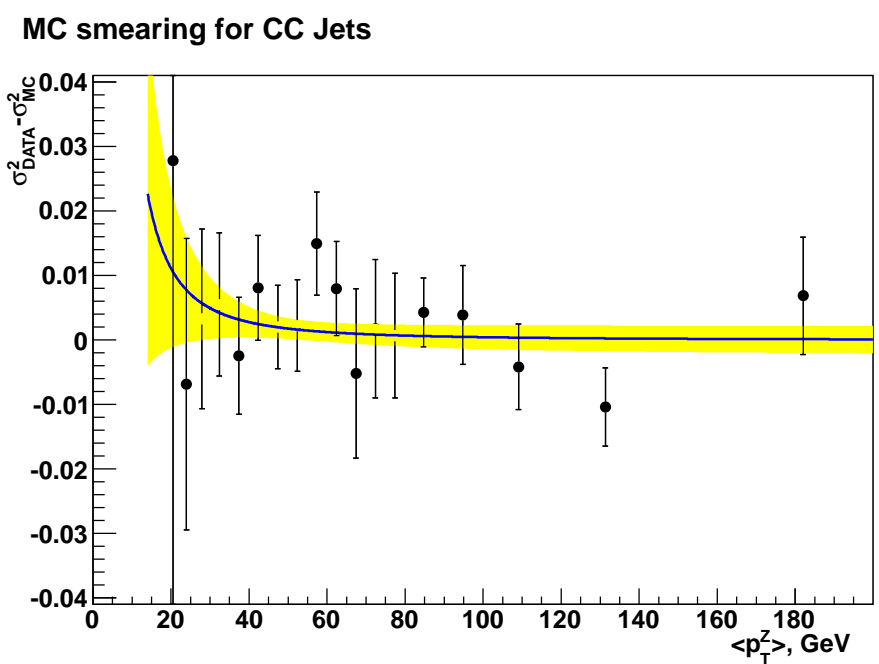

Figure 4.7: Square of the width of the smearing factor Gaussian as function of $p_{T}^{Z}$ for CC jets. The parametrization and the statistical error are represented by the blue curve and the yellow band.

\section{Shifting}

The imbalance between transverse energies of the $Z / \gamma$ and jet is provided by the mean $<\Delta S\rangle_{\text {Gauss }}$ of the Gaussian in the $\Delta S$ distribution. Fig. 4.8 shows the difference in the imbalances obtained in data and simulation $<\Delta S\rangle_{\text {Data }}-\langle\Delta S\rangle_{M C}$, which presents the data-MC relative jet energy scale (JES).

The fact that the data and MC transverse energies do not coincide results from different effective jet reconstruction thresholds and from different amounts of out-of-cone radiation in data and MC. To correct for this difference, the relative JES is parameterize as a function of $p_{T}^{\gamma / Z}$ and a shifting factor extracted from the fit is applied to the jet transverse energy in 
MC shifting for CC Jets

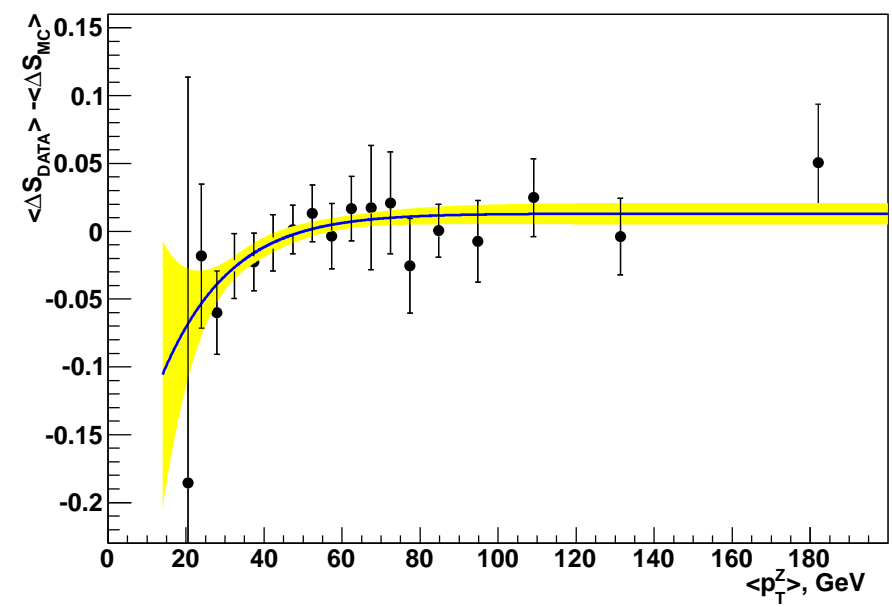

Figure 4.8: The shifting factor as function of $p_{T}^{Z}$ for CC jets. The parametrization and the associated error from the fit are represented by the blue curve and the yellow band.

MC.

Similar to the JES correction, the shifting correction also depends on the flavor of the partons initiating jets. Figure 4.9 shows the difference in Gaussian means between data and $\mathrm{MC}$ as a function of jet $p_{T}$ in $Z+1$ jet events for quark jets (primarily $u, d$ or $s$ quarks) and gluons. The data-MC relative JES is consistent with 0 for quark jets, while tending to be shifted downwards for gluon jets. The b-jets are know to be broader than light quark jets and that makes them more gluon-like. Therefore, the shifting correction is effectively applied to those physics processes with gluon or b-quark dominated final states.

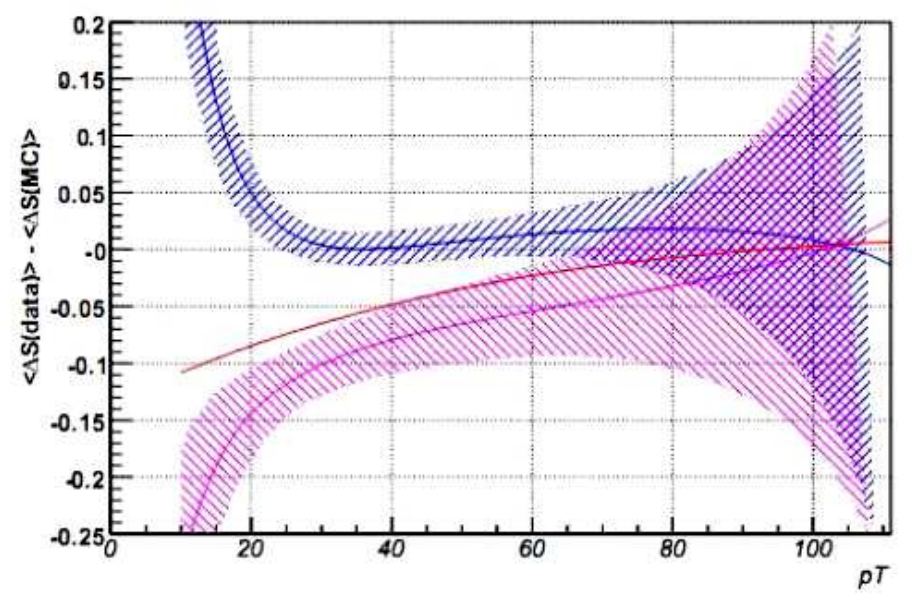

Figure 4.9: Relative JES of data and MC for quark (blue) and gluon (magenta) jets. The red line shows the nominal JSSR jet shifting for an ensemble of jets with both quark and gluon components. Hashed lines represent statistical errors. 
Undergoing the smearing and shifting procedures, the corrected jet $p_{T}$ is obtained by first smearing simulated jets according to the parameterization shown in Fig. 4.7, and then their energies are shifted according to the parameterization shown in Fig. 4.8:

$$
p_{T}^{\text {corr }}=p_{T}+p_{T}\left[\left(\operatorname{shift}\left(p_{T}\right)+\operatorname{Gauss}\left(0, \operatorname{smear}\left(p_{T}\right)\right)\right],\right.
$$

where $\operatorname{shift}\left(p_{T}\right)$ is the shifting factor and $\operatorname{Gauss}\left(0, \operatorname{smear}\left(p_{T}\right)\right)$ is a gaussian function centered at 0 and with a width shown in Fig. 4.7 as a function of $p_{T}$.

\section{Removal}

The fitted efficiency turn-on curves displayed in Fig. 4.10 present the dependence of jet reconstruction and identification efficiency on the jet $p_{T}$ in various calorimeter regions [69]. In both data and simulation, the full efficiency is reached at $\sim 15 \mathrm{GeV}$, which is taken as the threshold for jet removal. The jets with $p_{T}<15 \mathrm{GeV}$ are randomly removed from the MC to match the efficiency in data [68].
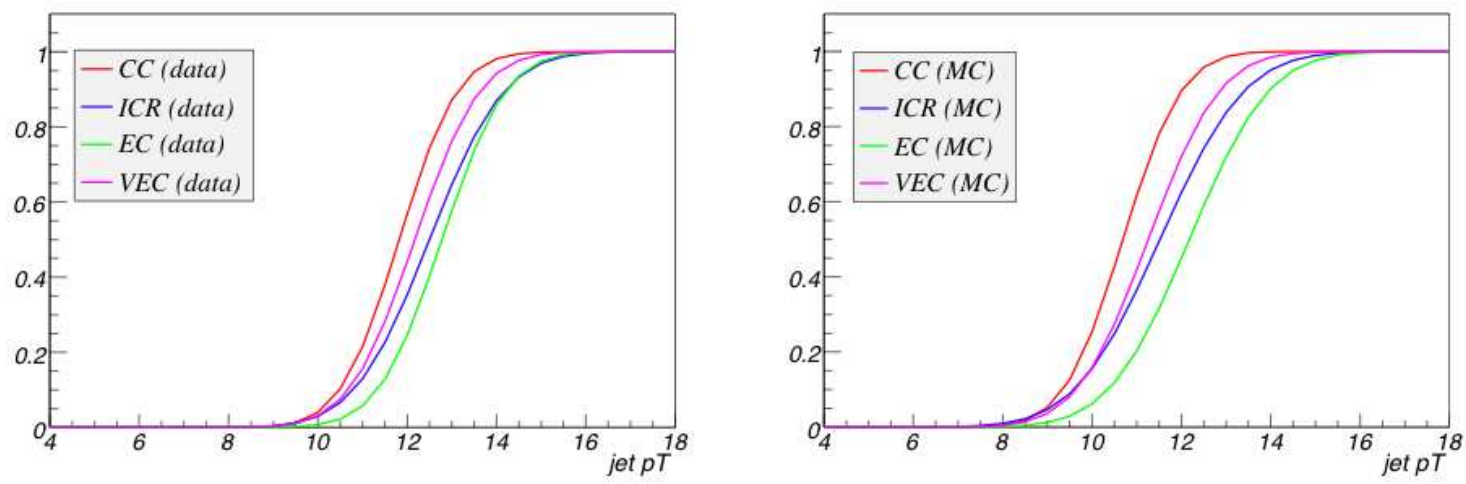

Figure 4.10: Average efficiency turn-on functions represented for different calorimeter regions fo data (left) and simulation (right).

\subsubsection{Missing Transverse Energy}

The presence of neutrinos in the final state of an event at the detector level is inferred from the missing transverse energy, which is the energy imbalance of an event in the transverse plane perpendicular to the beam. The raw $\mathbb{E}_{\mathrm{T}}$, which results from physics objects that escape the detector without energy deposits like neutrinos or from instrumental sources such as a calorimeter noise and physics object resolution, is measured by vectorially summing the calorimeter cells transverse energy:

$$
{\overrightarrow{E_{T}}}^{\text {raw }}=-\sum^{\text {cells }} \overrightarrow{E_{T}}
$$


The cells from the coarse hadronic layers of the calorimeter are not included in the sum since they suffer from relatively high noise (usually have higher noise and negative energy cells). The energy and momentum corrections applied to EM objects, muon and jets are propagated to the measured $\mathrm{E}_{\mathrm{T}}$ and the corrected $\mathrm{E}_{\mathrm{T}}$ is obtained by:

$$
\overrightarrow{E_{T}}=-\sum{\overrightarrow{E_{T}}}^{\text {collected }}-\sum^{\text {muons }} \overrightarrow{p_{T}}
$$

where $\sum \overrightarrow{p_{T}}$ is the sum of muon transverse momentum measured by the muon spectrometer.

\subsection{5 $b$-jet Identification}

Since the $H \rightarrow b \bar{b}$ decay mode is the most important search at the Tevatron for the SM Higgs boson with $M_{H}<135 \mathrm{GeV}$, identifying jets initiated from $b$-quarks (referred to as $b$-jet identification or $b$-tagging) is the main key for Higgs physics at the $\mathrm{D} \varnothing$. The facts that the mass of the b-quark is substantially larger than that of light flavor quarks (u, d, s, c) and the life time of the b-quark is sufficiently long for b-hadrons to be formed and travel a few millimeters before the b-quark decays lead to special signatures for b-jets observed in the detector. Various algorithms have been developed in the $\mathrm{D} \varnothing$ collaboration to exploit the properties of b-jets in order to detect their presence. The performance of identifying b-jets is improved significantly by using a neural network (NN) tagger to combine information obtained from different $b$-tagging algorithms into a final discriminant to distinguish b-jets from other jet flavors. Details about the $b$-tagging methods developed by the $\mathrm{D} \varnothing$ experiment are described in Ref. [70].

\section{$b$-jet properties}

The long lifetime and fragmentation properties of $b$-quarks allow us to use precise tracking information to identify $b$-jets though their decay products. The secondary vertex constructed from the tracks produced by the $b$-hadron decay products and the impact parameter (distance of closest approach of tracks to the primary vertex) are important features considered in $b$-tagging algorithms. Furthermore, $b$-hadron decays often lead to the production of high momentum leptons, which can provide easy access to samples with enhanced b-jet content. An illustration for reconstructing the secondary vertex and measuring the impact parameter of a b-jets is shown in Fig. 4.11.

\section{$b$-tagging prerequisites}

The requirements for a jet to be taggable, i.e., for it to be further investigated by the $b$ tagging algorithms, is that it must contain at least two tracks where each track has at least one hit in the SMT, the "seed" track must have $p_{T}>1 \mathrm{GeV}$ while the other track must have $p_{T}>0.5 \mathrm{GeV}$, and the distance of each track to the primary vertex must be $<2 \mathrm{~mm}$ in the transverse plane and $<4 \mathrm{~mm}$ in the $\mathrm{z}$ direction. 


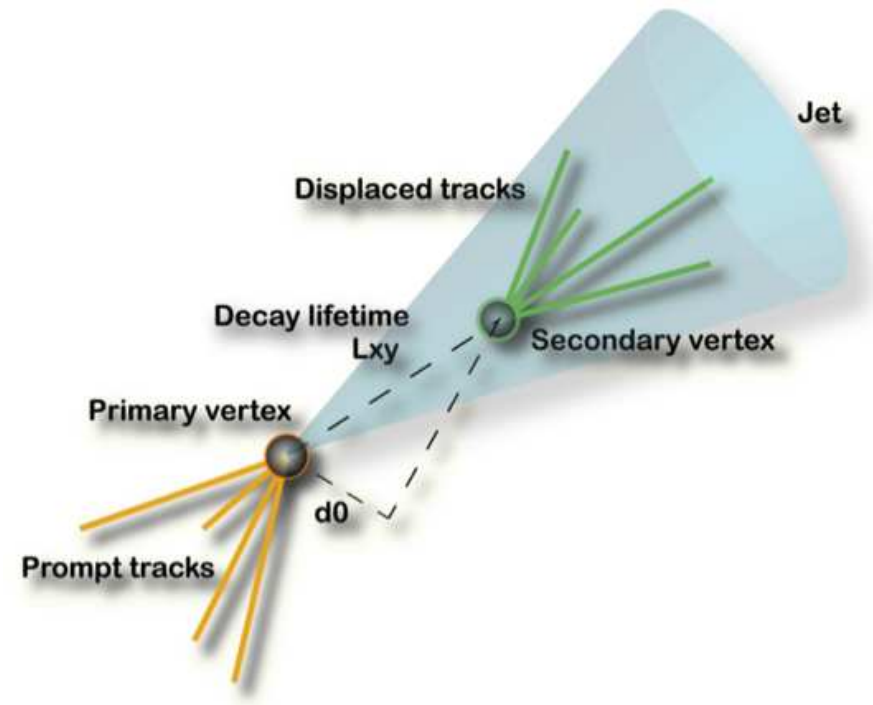

Figure 4.11: Reconstructed secondary vertex and impact parameter (d0) in a b-jet.

The efficiency for taggability is defined as the ratio of the number of taggable jets over the number of calorimeter jets. It accounts for variations in detector acceptance and track reconstruction efficiencies at different locations of the PV and is determined as a function of jet kinematics $\left(p_{T}\right.$ and $\left.\eta\right)$ and the z coordinate of the PV. To optimize the use of geometrical correlations between $\eta$ and $\mathrm{z}$, the taggability is parametrized in $z^{\prime} \equiv|z| \operatorname{sign}(\eta \cdot z)$ as shown in Fig. 4.12.

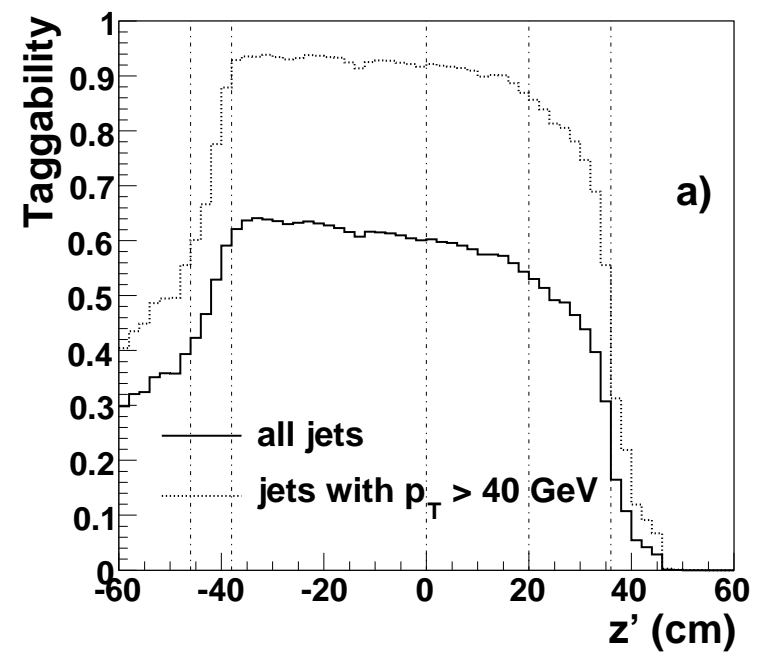

Figure 4.12: Taggability as a function of $z^{\prime}$. 


\section{$b$-tagging algorithms}

Several algorithms to identify b-jets exploiting the long lifetime and decayed products of b-quarks and have been developed in the $\mathrm{D} \varnothing$ Collaboration. They are based entirely on tracking and vertexing of the charged particles.

\section{- The secondary vertex tagger (SVT)}

The SVT is the most intuitive $b$-tagging algorithm which exploits the fact that bhadron decays give rise to multiple charged particles emanating from the b-hadron decay point. It is developed based on the reconstruction of this decay point and requirement of the presence of a secondary vertex. The algorithm starts from selecting tracks associated with a taggable jet and then requires that a number of tracks are extrapolated to the same point in three dimensions. Secondary vertices are associated with the calorimeter jet if $\Delta R($ vertex, jet $)<0.5$, where the vertex direction is computed as the difference of the secondary and primary vertex position.

\section{- The jet lifetime probability tagger (JLIP)}

The JLIP algorithm combines the impact parameters of all tracks associated with a calorimeter jet into a single variable, the jet lifetime probability $\mathcal{P}_{J I L P}$, which can be interpreted as the confidence level that all tracks in a jet originate from the selected primary interaction point. Jets from light quark fragmentation are expected to have an uniform $\mathcal{P}_{J I L P}$ distribution between 0 and 1 , while a peak at close to 0 is expected in $\mathcal{P}_{J I L P}$ distribution of jets from b- or c-quarks. A jet is tagged as $b$-jet if the $\mathcal{P}_{J I L P}$ is below an upper threshold which is set based on the signal efficiency and background rejection designed for a given analysis.

- The counting signed impact parameter tagger (CSIP) The CSIP algorithm selects "good" tracks with a certain quality within a $\mathcal{R}=0.5$ cone around the jet axis and computes the signed impact parameter significance $S_{d}$ for each selected tracks [70]:

$$
S_{d}=\frac{I P_{s}}{\sigma\left(I P_{s}\right)}
$$

where $I P_{s}$ is the signed impact parameter and $\sigma\left(I P_{s}\right)$ is the uncertainty on the measurement of $I P_{s}$. A jet is considered to be b-jet by the CSIP tagger if there are at

least two good tracks with $\frac{S d}{a}>3$ or at least three good tracks with $\frac{S d}{a}>2$, where $a$ is a scaling factor. The choice of $a$ determines the $b$-tagging efficiency and mistag rate of the algorithm.

\section{The Neutral Network (NN) b-tagger}

The $b$-tagging algorithms described above exploit different features of the b-hadron and perform separately to distinguish b-jets from other jet flavors. To improve the tagging 
efficiency with respect to any single algorithm, the NN $b$-tagger combines discriminating information provided by the SVT, JLIP and CSIP tagging algorithms into a single final discriminant to separate b-jets from light jets (where "light" stands for uds-quark or gluon). The NN was trained on simulated light flavor and $b \bar{b}$ samples using nine input variables. Six of the variables are based on the secondary vertex reconstructed by the SVT algorithm, and the remaining three variables are obtained using the JLIP and CSIP algorithms. The description of these variables is given in Ref. [70]:

- SVT $S_{x y}$ : the decay length significance (the decay length in the transverse plane divided by its uncertainty) of the secondary vertex with respect to the primary vertex.

- $\operatorname{SVT} \chi_{d o f}^{2}$ : the $\chi^{2}$ per degree of freedom of the secondary vertex fit.

- SVT $N_{t r k}$ : the number of tracks used to reconstruct the secondary vertex.

- SVT $m_{v t x}$ : the mass of the secondary vertex.

- SVT $N_{v t x}$ : the number of secondary vertices reconstructed in the jet.

- SVT $\Delta R$ : the distance in $(\eta, \phi)$ space between the jet axis and the difference between the secondary and primary vertex positions.

- JLIP $\mathcal{P}_{J L I P}$ : the jet lifetime probability computed by JLIP algorithm.

- JLIP $\mathcal{P}_{\text {RedJLIP }}: \mathcal{P}_{J L I P}$ re-calculated with the track with the highest significance removed from the calculation.

- CSIP $N_{C S I P}$ : a combined variable based on the number of tracks with an impact parameter significance greater than an optimized value.

The output from the optimized NN $b$-tagger on $b \bar{b}$ and light-flavor simulated jets given in Fig. 4.13 shows a significant separation between the signal and background samples.

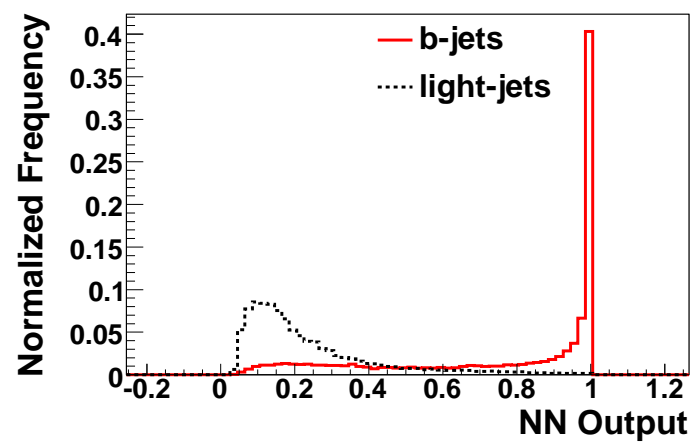

Figure 4.13: The NN output for light-flavor jets (dashed line) and b-jets (continuous line) with $p_{T}>15 \mathrm{GeV}$ and $|\eta|<2.5$ in simulated QCD events. Both distributions are normalized to unity. 
The larger cut values on the NN output set a tighter $b$-tagging working point. Table 4.3 lists several operating points with associated $b$-tagging efficiency and light-flavor mistag rate (fake rate) for jets in $\mathrm{CC}$ region.

\begin{tabular}{||c|c|c|c||}
\hline \hline Operating point & Loose & Medium & Tight \\
\hline \hline NN output & 0.45 & 0.65 & 0.77 \\
b-jet efficiency & $60.8 \%$ & $53.7 \%$ & $47.6 \%$ \\
Fake rate & $2.02 \%$ & $0.96 \%$ & $0.55 \%$ \\
\hline \hline
\end{tabular}

Table 4.3: The $b$-tagging operating points with associated efficiency and fake rate. 


\section{5}

\section{Signal Prediction and Background Estimation}

\subsection{Data Samples}

The search for SM Higgs boson production and decay processes that can lead to the final states containing leptons and jets presented in this thesis uses the full data set corresponding to $9.7 \mathrm{fb}^{-1}$ of integrated luminosity of $p \bar{p}$ collisions at a center-of-mass energy of $\sqrt{s}=$ $1.96 \mathrm{TeV}$ collected with the $\mathrm{D} \varnothing$ detector.

The most crucial step in our analyses is selecting a sub-dataset of events which have topologies similar to the signatures of the targeted SM Higgs boson signals. However, various SM processes can mimic the expected signal signatures, and thus need to be considered as potential background. In order to find the SM Higgs boson or exclude its existence, it is essential to have deep understanding of the differences in the properties of the SM Higgs signal and background processes at the detector level. Therefore, MC simulated signal and background samples are produced based on SM prediction to model compositions of data. The differences in kinematics of simulated signal and background events are then exploited to build a mathematical tool for differentciating signal events from background events in data.

There are two major data subsamples collected with the $\mathrm{D} \varnothing$ detector over two different time periods. The first is the RunIIa data set taken from August 2002 until the 2006 shutdown, and the second is the RunIIb data set taken from June 2006 to September 2011. Since important detector and trigger updates have been made between RunIIa and RunIIb, two different sets of simulated MC samples have been used RunIIa and RunIIb to properly describe the data. 


\subsection{Monte Carlo Generators}

Physics events recorded in the data samples are created from $p \bar{p}$ collisions through the following processes:

- Initially two beams of protons and antiprotons are coming towards each other. Each proton and antiproton involved in a $p \bar{p}$ collision is characterized by a set of parton distribution functions (PDF) which defines the partonic substructure of the proton and antiproton in terms of composition and momentum distribution.

- As $p$ and $\bar{p}$ come into contact, partons from the $p$ or $\bar{p}$ may start a sequence of branchings with initial-state radiation (ISR) and build up initial-state showers.

- Two partons from initial-state showers originating from a $p \bar{p}$ pair undergo a hard scatter process, and then a number of outgoing partons are produced. The nature of this process determines the main characteristics of the event.

- The outgoing partons can start branching with final-state radiation (FSR) and build up final-state showers. The gluons created in the shower can produce further quark pairs, and that increases particle multiplicity in the final state of the event.

- The outgoing quarks and gluons are not directly observable, because they fragment into neutral hadrons. Most of the hadrons produced during the fragmentation are unstable and decay to final state particles forming jets, which finally are identified by the detector. The process wherein the coloured partons are transformed into jets of colourless hadrons, photons and leptons is called hadronization.

The evolution of a physics event as described above can be modeled by different Monte Carlo generators, and the resulting observable signals can be studied using detailed detector smulations. A brief description of these event generators is given in this chapter.

\subsubsection{PYTHIA}

The PYThia Monte Carlo is a general-purpose event generator [71]. It allows generation of complete events within the bounds of current understanding of the underlying physics. The PYTHIA generator as configured for these studies uses the CTEQ6L1 [72] leading order parton distribution functions to model the initial partonic states for the hard parton scattering processes. The parton-shower approach, where the probability for a parton to branch is given by evolution equations, is employed to model the ISR and FSR processes. The hadronization process takes place at low $Q^{2}$ where QCD perturbation theory breaks down. It is codified in PYTHIA based on the phenomenological model called "String Fragmentation" with the assumption of linear confinement [71]. 


\subsubsection{ALPGEN}

The ALPGEN generator is designed for the generation of complex and high-multiplicity hard scattering final states in hadronic collisions. In this program, the tree level scattering amplitude for multi-parton final states is computed based on a recursive numerical Legendre transform of the effective action starting from the SM Lagrangian [73]. Using the CTEQ6L1 parton distribution functions, ALPGEN provides the most accurate modeling for the underlying hard processes of major backgrounds in our analyses, which have final states containing a combination of electroweak bosons along with multiple quarks and gluons. However, ALPGEN itself does not handle the showering and hadronization processes. To complement the calculation of parton-level matrix elements with the evaluation of the full hadronic structure of the final state, the parton-level calculations given by ALPGEN are merged with the partonic evolution through interfacing with shower MC programs such as PYTHIA, HERWIG Or ISAJET.

\subsubsection{SingleTop}

The SingleTop generator is a matrix element generator designed for simulating electroweak single top-quark production in the next leading order (NLO) approximation [74]. The spincorrelation effects in the single top-quark production are also considered in this program. Subsequent parton shower and hadronization are provided via an interface with PYTHIA.

\subsubsection{MCFM}

MCFM is a parton-level Monte Carlo program [75]. It is designed to calculate cross-sections for various femtobarn-level processes at hadron colliders. For most processes, matrix elements are included to the next-to-leading order calculation and spin correlations are fully incorporated.

\subsubsection{MLM Scheme}

The MLM matching scheme is a procedure for combining tree-level matrix element generators with parton showers. The matching criteria developed in the MLM algorithm eliminate the double counting of configurations where jets can arise from both the higher-order parton level calculation and from hard emission during the shower evolution [76]. To properly simulate events with high jet multiplicity in our analyses, the MLM algorithm has been used to merge matrix elements of hard underlying processes generated by ALPGEN and the subsequent development of the hard partons into jets of hadrons modeled by PYTHIA. 


\subsection{Detector Simulation}

In the real world, the collider machine produces interactions and the events are observed at the detector. Information about an event, particles produced and their momentum, is reconstructed based on electronic signals from detector systems, such as sillicon and scintillating fiber tracking systems, calorimeters and wire chambers.

In the Monte Carlo world, the generators play the role of collider machine producing events. The behaviour of the $\mathrm{D} \varnothing$ detector is fully simulated in two programs, the $\mathrm{D} \varnothing$ detector simulation DØgstar based on the detector material simulation package GEANT and the simulation of $\mathrm{D} \varnothing$ detector electronics and pileup interactions by DØsim. The outputs of MC generators are used as inputs for DØgstar program and then passed to DØsim. The DØgstar program models how particles produced by the event generator interact with magnetic fields and material in the detector. The DØsim program models detector noise, residual activity from previous beam crossings and contributions from the presence of additional $p \bar{p}$ interactions. The output of the detector simulation has exactly the same format as the real data recorded by the detector, and these events are fed into the same program for event reconstruction as collider data.

\subsection{Simulated Event Samples}

A brief description of the main Higgs boson signal and background processes in the $Z H \rightarrow$ $\ell \ell b b$ and $\ell \nu j j$ analyses was given in Sec. 2.4 of Chapter 2. Besides the main signal processes introduced, we also include signal components not specifically targeted but which fall in our acceptance criteria nonetheless. The following section presents details of the simulation of the signal and background contributions in each analysis.

\subsubsection{Simulated signal processes}

\section{Signal samples in $\ell \nu j j$ analysis}

In the $\ell \nu j j$ analysis, we account for all SM Higgs boson production with and decay processes that can lead to a final state containing exactly one charge lepton, a significant imbalance in transverse energy $\left(\mathbb{E}_{\mathrm{T}}\right)$, and two or more jets. The considered signal processes are categorized by the production mode as the following:

\section{- Associated production of a Higgs boson with a vector boson}

(i) $W H \rightarrow \ell \nu b b$ : This is the most important signal process in the low Higgs mass region, where the Higgs boson is produced in association with a $W$ boson, the SM Higgs boson decays to a $b \bar{b}$ pair and the $\mathrm{W}$ boson decays leptonically.

(ii) $V H \rightarrow V V V^{*} \rightarrow \ell \nu j j j j$ : In this process, the Higgs boson is also produced in association with a vector boson $(W$ or $Z$ ) and then decays into a pair of vector 
bosons. One vector boson decays leptonically, and two other vector bosons decay hadronically producing four jets. The $V H \rightarrow V V V^{*} \rightarrow \ell \nu j j j j$ process contributes to both low mass and high mass regions.

(iii) Additional topologies that are also considered in this context are $W H \rightarrow \ell \nu c \bar{c}$, $W H \rightarrow \ell \nu \tau^{+} \tau^{-}, Z H \rightarrow l^{+} l^{-} b \bar{b}$. However, these three signal processes just play a minor role in $\ell \nu j j$ analysis, due to the low decay rates of $H \rightarrow \tau^{+} \tau^{-}, c \bar{c}$, and that, the contributions from $Z H \rightarrow l^{+} l^{-} b \bar{b}$ only arises when one lepton in the final state is mis-identified and significant $\mathrm{E}_{\mathrm{T}}$ results from this mis-identification and/or poor jet energy resolution.

\section{- Gluon fusion production}

(i) $g g \rightarrow H \rightarrow W W^{*} \rightarrow \ell \nu j j$ : The Higgs boson is produced through the main production channel, $g g \rightarrow H$, and then decays into a pair of $\mathrm{W}$ bosons, where one $\mathrm{W}$ boson decays leptonically and the other decays hadronically. This is the dominant signal production process in the high Higgs mass region and it contributes to low Higgs mass region as well.

(ii) $g g \rightarrow H \rightarrow Z Z^{*} \rightarrow l^{+} l^{-} j j$ : The Higgs boson in this process decays into a pair of $\mathrm{Z}$ bosons, where one $\mathrm{Z}$ boson decays hadronically and the other decays leptonically (with only one lepton identified). The contribution of this channel is rather small.

\section{- Vector boson fusion production}

(i) $V B F \rightarrow H \rightarrow V V^{*} \rightarrow \ell \nu j j$ : The Higgs boson is produced through the vector boson fusion mechanism and then decays into a pair of $W$ or $Z$ bosons, where one vector boson decays leptonically (with exactly one identified lepton). The contribution of this signal process is minor due to the small cross section of the SM Higgs production through vector boson fusion.

\section{Signal samples in $Z H \rightarrow \ell \ell b b$ analysis}

- The $Z H \rightarrow \ell \ell b b$ analysis requires the final state to have two isolated charged leptons (either electrons or muons), at least two jets and at least one jet must satisfy a tight b-tagging requirement. Besides the main signal process $Z H \rightarrow l^{+} l^{-} b \bar{b}$ (where the Higgs boson is produced in association with a $\mathrm{Z}$ boson, then the Higgs boson decays into a pair of b-quarks and the $\mathrm{Z}$ boson decays into a pair of charged letons), we also consider $Z H \rightarrow l^{+} l^{-} c \bar{c}$ and $Z H \rightarrow l^{+} l^{-} \tau^{+} \tau^{-}$signal processes. The $Z H \rightarrow l^{+} l^{-} b b$ signal component accounts for more than $90 \%$ of the total signal yields.

All SM Higgs signal processes considered in our analyses are simulated with the Monte Carlo event generator PyTHIA at leading order. The signal samples involving $H \rightarrow b \bar{b}, H \rightarrow c \bar{c}$ or $H \rightarrow \tau^{+} \tau^{-}$decays are produced in the mass range $90-200 \mathrm{GeV}$ in $5 \mathrm{GeV}$ steps. The signal samples involving $H \rightarrow V V^{*}$ decays, which dominate the sensitivity of high mass searches, 


\begin{tabular}{||crrrrrrrcc||}
\hline \hline$M_{H}(\mathrm{GeV})$ & $\sigma_{g g \rightarrow H}$ & $\sigma_{W H}$ & $\sigma_{Z H}$ & $\sigma_{V B F}$ & $\mathcal{B}_{(H \rightarrow b \bar{b})}$ & $\mathcal{B}_{(H \rightarrow c \bar{c})}$ & $\mathcal{B}_{\left(H \rightarrow \tau^{+} \tau^{-}\right)}$ & $\mathcal{B}_{\left(H \rightarrow W^{+} W^{-}\right)}$ & $\mathcal{B}_{(H \rightarrow Z Z)}$ \\
\hline 90 & 2442 & 394.7 & 224.0 & 118.2 & 81.2 & 3.78 & 8.41 & 0.21 & 0.042 \\
95 & 2101 & 332.1 & 190.3 & 108.8 & 80.4 & 3.73 & 8.41 & 0.47 & 0.067 \\
100 & 1821 & 281.1 & 162.7 & 100.2 & 79.1 & 3.68 & 8.36 & 1.11 & 0.113 \\
105 & 1584 & 238.7 & 139.5 & 92.3 & 77.3 & 3.59 & 8.25 & 2.43 & 0.215 \\
110 & 1385 & 203.7 & 120.2 & 85.2 & 74.5 & 3.46 & 8.03 & 4.82 & 0.439 \\
115 & 1215 & 174.5 & 103.9 & 78.7 & 70.5 & 3.27 & 7.65 & 8.67 & 0.873 \\
120 & 1072 & 150.1 & 90.2 & 72.7 & 64.9 & 3.01 & 7.11 & 14.3 & 1.60 \\
125 & 949 & 129.5 & 78.5 & 67.1 & 57.8 & 2.68 & 6.37 & 21.6 & 2.67 \\
130 & 842 & 112.0 & 68.5 & 62.1 & 49.4 & 2.29 & 5.49 & 30.5 & 4.02 \\
135 & 750 & 97.2 & 60.0 & 57.5 & 40.4 & 1.87 & 4.52 & 40.3 & 5.51 \\
140 & 670 & 84.6 & 52.7 & 53.2 & 31.4 & 1.46 & 3.54 & 50.4 & 6.92 \\
145 & 600 & 73.7 & 46.3 & 49.4 & 23.1 & 1.07 & 2.62 & 60.3 & 7.96 \\
150 & 539 & 64.4 & 40.8 & 45.8 & 15.7 & 0.725 & 1.79 & 69.9 & 8.28 \\
155 & 484 & 56.2 & 35.9 & 42.4 & 9.18 & 0.425 & 1.06 & 79.6 & 7.36 \\
160 & 432 & 48.5 & 31.4 & 39.4 & 3.44 & 0.159 & 0.397 & 90.9 & 4.16 \\
165 & 383 & 43.6 & 28.4 & 36.6 & 1.19 & 0.055 & 0.138 & 96.0 & 2.22 \\
170 & 344 & 38.5 & 25.3 & 34.0 & 0.79 & 0.036 & 0.092 & 96.5 & 2.36 \\
175 & 309 & 34.0 & 22.5 & 31.6 & 0.61 & 0.028 & 0.072 & 95.8 & 3.23 \\
180 & 279 & 30.1 & 20.0 & 29.4 & 0.50 & 0.023 & 0.059 & 93.2 & 6.02 \\
185 & 252 & 26.9 & 17.9 & 27.3 & 0.39 & 0.018 & 0.046 & 84.4 & 15.0 \\
190 & 228 & 24.0 & 16.1 & 25.4 & 0.32 & 0.015 & 0.038 & 78.6 & 20.9 \\
195 & 207 & 21.4 & 14.4 & 23.7 & 0.27 & 0.013 & 0.032 & 75.7 & 23.9 \\
200 & 189 & 19.1 & 13.0 & 22.0 & 0.24 & 0.011 & 0.029 & & 74.1 \\
\hline \hline
\end{tabular}

Table 5.1: The production cross sections (in fb) and decay branching fractions (in \%) for SM Higgs boson.

are produced in the mass range $100-200 \mathrm{GeV}$ in $5 \mathrm{GeV}$ steps. The signal samples generated at leading order (LO) production rate are then normalized to the most recent higher-order prediction. The calculations of associated production cross sections, $W H$ and $Z H$, are performed at next-to-next-to-leading-order (NNLO) precision in QCD and next-to-leadingorder (NLO) precision in electroweak corrections [77]. The cross section of gluon-gluon fusion production $\left(\sigma_{g g \rightarrow H}\right)$ is calculated at NNLO in QCD with next-to-next-to-leading-log (NNLL) accuracy of soft gluon resummation [78]. The vector-boson fusion cross section is computed at NNLO in QCD [79], and the electroweak correction to VBF production is computed with the HAWK program [80]. The Higgs boson decay branching ratio predictions are taken from HDECAY $[81,82]$. The inclusive Higgs boson production cross sections as well as the relevant decay branching fractions considered in our analyses are listed in Table 5.1.

\subsubsection{Simulated Background processes}

For both $Z H \rightarrow \ell \ell b b$ and $\ell \nu j j$ analyses, background contributions from most SM processes are estimated by MC simulation, except for the QCD multijet background which is determined by a data-driven method. Details about generating and normalizing simulated backgrounds will be described in the following sections. 


\section{$W+$ jets and $Z+$ jets backrounds}

The dominant background for the $\ell \nu j j$ and $Z H \rightarrow \ell \ell b b$ anylyses is from $W+$ jets and $Z+$ jets processes, respectively. The $Z+$ jets process can also be a background component of the $\ell \nu j j$ analysis if one charged leptons from $\mathrm{Z}$ decays is unidentified and significant missing transverse energy results from this and/or poor jet energy resolution. In fact, $Z+$ jets is the second largest background in the $\ell \nu j j$ analysis. The $W+$ jets and $Z+$ jets $(V+$ jets $)$ samples are generated at LO with the matrix-element generator ALPGEN interfaced with PYTHIA for subsequent parton showering and hadronization. The MLM scheme is applied to avoid overestimating the probability of further partonic emissions in PYTHIA.

Separate $W / Z+$ jets sub-samples with different number of partons or different parton flavor in the final state are produced. The $W / Z+$ jets sample made of events with only light partons ( $\mathrm{u}, \mathrm{d}, \mathrm{s}$ quarks or gluon) is called $W / Z+\mathrm{LP}$, and the sample made of events with heavy-flavor partons (b or c quarks) is called $W / Z+\mathrm{HF}$. The $W / Z+\mathrm{HF}$ sample includes $W / Z+b \bar{b}$ and $W / Z+c \bar{c}$. To ensure the orthogonality among these sub-samples, a procedure called Heavy Flavor (HF) skimming is applied to remove events with $b$ or $c$ quarks from $W / Z+\mathrm{LP}$ samples and events with $c$ quarks $W / Z+b \bar{b}$ samples.

\begin{tabular}{||c|c|c||}
\hline \hline Sample & K-factor & $\boldsymbol{K}_{\mathbf{H F}}$ \\
\hline$W / Z+\mathrm{LP}$ & 1.3 & 1 \\
$W+b \bar{b}$ & 1.3 & 1.47 \\
$Z+b \bar{b}$ & 1.3 & 1.52 \\
$W / Z+c \bar{c}$ & 1.3 & 1.67 \\
\hline \hline
\end{tabular}

Table 5.2: K-factors and $\boldsymbol{K}_{\mathbf{H F}}$ for $\boldsymbol{W} / \boldsymbol{Z}+$ jets processes.

The LO cross section of the inclusive $W / Z+$ jets samples generated by ALPGEN is scaled to the NNLO calculation by applying a correction K-factor [83]. Additional correction factors, $K_{\mathrm{HF}}$, calculated at NLO from MCFM are applied to heavy-flavor samples [84]. The heavy flavor scale factor for the $W / Z+b \bar{b}$ samples is computed as:

$$
K_{\mathrm{HF}}^{\mathrm{Vbb}}=\frac{\sigma_{(\mathrm{NLO} \mathrm{Vb \overline {b }})} / \sigma_{(\mathrm{LO} \mathrm{Vb} \bar{b})}}{\sigma_{(\mathrm{NLO} \mathrm{V}+\mathrm{LP})} / \sigma_{(\mathrm{LO} \mathrm{V}+\mathrm{LP})}},
$$

where $\left.\sigma_{(\mathrm{NLO}} \mathrm{Vb \overline {b }}\right)$ and $\left.\sigma_{(\mathrm{LO}} \mathrm{Vb \overline {b }}\right)$ are NLO and LO $W+\mathrm{b} \overline{\mathrm{b}}+0 \mathrm{lp}$ cross sections from MCFM, and $\left.\sigma_{(\mathrm{NLO}} \mathrm{V}+\mathrm{LP}\right)$ and $\left.\sigma_{(\mathrm{LO}} \mathrm{V}+\mathrm{LP}\right)$ are NLO and LO $W+2 \mathrm{lp}$ cross sections from MCFM. The heavy flavor scale factor for the $W / Z+c \bar{c}$ samples are calculated using the same method. The K-factor and $K_{\mathrm{HF}}$ applied to $W / Z+$ jets samples in our analyses are listed in Table 5.2.

\section{$t \bar{t}$ and single top backrounds}

Background from top quark pair and single top quark processes are respectively generated with ALPGEN and SINGLETOP using the CTEQ6L1 parton distribution functions and inter- 
faced with PYTHIA. The LO cross section of top quark pair production in the simulated sample is then normalized to approximate NNLO [85], while the cross section for single top quark production is normalized to the next-to-NNLO [86]. This higher-order correction in the production cross section results in a K-factor of 1.43 and 0.99 applied to the normalization of the $t \bar{t}$ and single top quark samples, respectively.

\section{Diboson backgrounds}

Diboson processes $(V V)$ are simulated by PYTHIA at the LO approximation. The cross sections of diboson production are then normalized to NLO calculations [87, 75]. The correction factors of 1.005, 1.061 and 1.030 are applied to $W W, W Z$ and $Z Z$, respectively.

A list of cross sections with the corresponding approximation for each background process is given in Table 5.3.

\begin{tabular}{||l|c|c||}
\hline \hline \multicolumn{1}{|c|}{ Sample } & Cross section $(\mathrm{pb})$ & Approximation \\
\hline$W(\rightarrow \ell \nu)+2$ jets inclusive & 509.03 & NNLO \\
$Z\left(\rightarrow l^{+} l^{-}\right)+2$ jets inclusive & 99.43 & NNLO \\
$t \bar{t}$ inclusive & 7.04 & NNLO \\
Single Top inclusive & 3.15 & NNLO \\
Diboson $(W W, W Z, Z Z)$ & 16.47 & NLO \\
\hline \hline
\end{tabular}

Table 5.3: Cross sections and order of accuracy of background processes.

\subsection{Estimation of Multijet Background}

\subsubsection{Multijet background modeling strategy}

The QCD multijet events do not contain isolated charged leptons in the final state, but they still can contribute to the background when jets are misidentified as leptons or leptons from decay products of jets are misidentified as isolated leptons. In general, the efficiency for a jet or its decay product to fake an isolated lepton and multijet events to pass all selection cuts to fall into the acceptance is small. But the cross section for QCD processes is large, therefore, the contribution from multijet events to the background (referred to as the MJ background) can not be ignored.

The multijet background in both $Z H \rightarrow \ell \ell b b$ and $\ell \nu j j$ analyses are estimated by the data-driven matrix method [88]. To derive the shape and the normalization of the multijet background, three data samples, tight, loose and loose-not-tight are defined. The tight sample, which is used for SM Higgs signal searching, is a selected sample with strict criteria applied on leptons. The loose sample is obtained by changing the lepton requirement to be less strict. The loose-not-tight sample is made of events from data in which the leptons pass the loose isolation requirement, but fail the tight requirement. It is a sub-sample of loose 
sample and orthogonal to the tight sample. The loose-not-tight data sample is composed of multijet events with jets faking loose leptons $\left(N_{l n t}^{Q C D}\right)$ and events with real charged leptons $\left(N_{l n t}^{\ell}\right)$. The total number of events in the loose-not-tight sample $\left(N_{l n t}\right)$ is:

$$
N_{l n t}=N_{l n t}^{Q C D}+N_{l n t}^{\ell}
$$

Using the same naming convention, similar relations for event components of tight and loose samples are written as:

$$
N_{\text {tight }}=N_{\text {tight }}^{Q C D}+N_{\text {tight }}^{\ell}, \quad N_{\text {loose }}=N_{\text {loose }}^{Q C D}+N_{\text {loose }}^{\ell},
$$

where $N_{\text {tight }}^{Q C D}, N_{\text {tight }}^{\ell}$ and $N_{\text {tight }}\left(N_{\text {loose }}^{Q C D}, N_{\text {loose }}^{\ell}\right.$ and $\left.N_{\text {loose }}\right)$ are the number of mutijet events, the number of events having a real chaged lepton and the total number of events in tight (loose) sample, respectively.

The relative efficiency $\epsilon_{L T}^{\ell}$ for a lepton passing loose requirements to subsequently pass the tight requirements and a relative probability, $P_{L T}^{M J}$, for a multijet event to pass these sequential selections are defined as:

$$
\epsilon_{L T}^{\ell}=\frac{N_{\text {tight }}^{\ell}}{N_{\text {loose }}^{\ell}}, \quad P_{L T}^{M J}=\frac{N_{\text {tight }}^{Q C D}}{N_{\text {loose }}^{Q C D}} .
$$

Using Eqn. (5.2), Eqn. (5.3) and Eqn. (5.4) we obtain:

$$
N_{\text {lnt }}=N_{\text {loose }}-N_{\text {tight }}=\left(1-P_{L T}^{M J}\right) \cdot N_{\text {loose }}^{Q C D}+\left(1-\epsilon_{L T}^{\ell}\right) \cdot N_{\text {loose }}^{\ell}
$$

From Eqn. (5.4 )and Eqn. (5.5) we then find:

$$
N_{\text {loose }}^{Q C D}=\frac{1}{1-P_{L T}^{M J}} \cdot N_{\text {loose }}-\frac{1}{\epsilon_{L T}^{\ell}} \cdot \frac{1-\epsilon_{L T}^{\ell}}{1-P_{L T}^{M J}} \cdot N_{\text {tight }}^{\ell} .
$$

We use Eqn. (5.4) and Eqn. (5.6) to estimate the number of multijet events in tight sample:

$$
N_{\text {tight }}^{Q C D}=\frac{P_{L T}^{M J}}{1-P_{L T}^{M J}} \cdot N_{\text {lnt }}-\frac{P_{L T}^{M J}}{\epsilon_{L T}^{\ell}} \cdot \frac{1-\epsilon_{L T}^{\ell}}{1-P_{L T}^{M J}} \cdot N_{\text {tight }}^{\ell} .
$$

From Eqn. (5.3) and Eqn. (5.7) we have:

$$
N_{\text {tight }}=\frac{P_{L T}^{M J}}{1-P_{L T}^{M J}} \cdot N_{l n t}+\left[1-\frac{P_{L T}^{M J}}{\epsilon_{L T}^{\ell}} \cdot \frac{1-\epsilon_{L T}^{\ell}}{1-P_{L T}^{M J}}\right] \cdot N_{\text {tight }}^{\ell} .
$$

The first term in Eqn. (5.7) containing $N_{l n t}$, which comes from the loose-not-tight sample, represents the shape of multijet background. The second term in Eqn. (5.7) containing $N_{\text {tight }}^{\ell}$ represents the real charged lepton contamination which leaks into the estimated multijet sample and needs to be subtracted to get an accurate estimation for multijet background. 
According to Eqn. (5.7) and Eqn. (5.8), the shape and the normalization of the multijet background can be obtained from the loose-not-tight data sample and the tight MC sample. We first construct a template sample that models the kinematics of multijet events by assigning each event in the loose-not-tight data sample a weight:

$$
w_{M J}=\frac{P_{L T}^{M J}}{1-P_{L T}^{M J}} .
$$

To account for the real electron contamination to the multijet template and get the correct modeling for the tight sample, the MC simulated background samples passing the tight selection need to be scaled down by:

$$
1-\frac{P_{L T}^{M J}}{\epsilon_{L T}^{\ell}} \cdot \frac{1-\epsilon_{L T}^{\ell}}{1-P_{L T}^{M J}} .
$$

\subsubsection{Derivation of $P_{L T}^{M J}$}

The probability $P_{L T}^{M J}$ is determined in the region $5<\mathrm{E}_{\mathrm{T}}<15 \mathrm{GeV}$ for events with a single lepton candidate, where the QCD contribution is dominant, and parameterized as a function of the event kinematics. For a given kinematic region, $P_{L T}^{M J}$ is derived from the ratio of the number of data events with a tight lepton and those with a loose lepton after subtracting for the expected MC contribution from SM backgrounds with real leptons:

$$
P_{L T}^{M J}=\frac{N_{\text {tight }}^{\text {Data }}-\left[N_{\text {tight }}^{V+j e t s}+N_{\text {tight }}^{T o p}+N_{\text {tight }}^{V V}\right]}{N_{\text {loose }}^{\text {Data }}-\left[N_{\text {loose }}^{V+j e t s}+N_{\text {loose }}^{T o p}+N_{\text {loose }}^{V V}\right]} .
$$

For electron channels, where the charged leptons in the final states are electrons, the probability $P_{L T}^{M J}$ is parametrized as function of lepton calorimeter detector $\eta$ :

$$
f(\eta)=a_{0}+a_{1}|\eta|^{2}+a_{2}|\eta|^{4}
$$

where $a_{n}$ are the fit parameters determined in each of lepton transverse momentum $p_{T}$ and $\Delta \phi\left(E_{\mathrm{T}}, \mathrm{j}\right)_{\min }$ bins. For muon channels, the probability $P_{L T}^{M J}$ is parametrized as a fourthorder polynomial function of lepton transverse momentum $p_{T}$ for different regions in muon detector $\eta$ and $\Delta \phi\left(\mathbb{E}_{\mathrm{T}}, \mu\right)$ :

$$
f\left(p_{T}\right)=b 1_{0}+b_{1}\left(p_{T}\right)^{2}+b_{2}\left(p_{T}\right)^{4} .
$$

Figure 5.1 shows examples of resulting fits for $P_{L T}^{M J}$ with the parameterization functions overlaid on the corresponding data, along with associated uncertainty bands. 

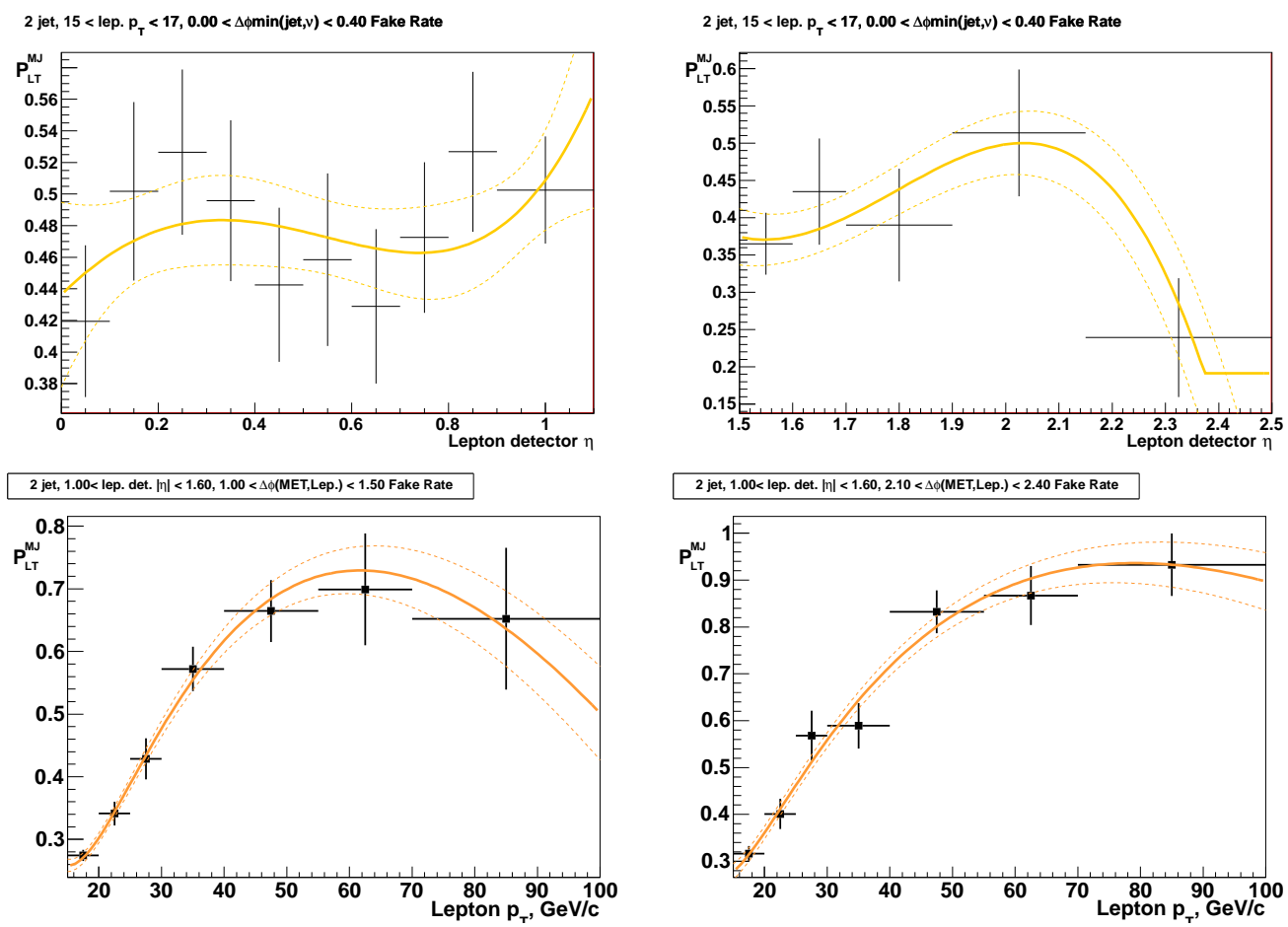

Figure 5.1: Examples of $P_{L T}^{M J}$ as a function of detector $|\eta|$ (lepton $p_{T}$ ) in different specific kinematic regions for electron (muon) channel. The plots for electron (muon) channel are on the top (bottom).

\subsubsection{Derivation of $\epsilon_{L T}^{\ell}$}

The lepton efficiencies for loose and tight samples are derived based on samples of $Z \rightarrow \ell \ell$ events as presented in Section II of Chapter 4. The efficiencies $\epsilon_{L T}^{\ell}$ are then determined from the ratio of measured lepton efficiencies for tight and loose samples. For an electron, the efficiency $\epsilon_{L T}^{\ell}$ is parametrized in bins of electron detector $|\eta|$ and electron $p_{T}$ bins, while for the muon the efficiency $\epsilon_{L T}^{\ell}$ is parametrized as a function of muon $p_{T}$ :

$$
f\left(p_{T}\right)=c_{0} \times \tanh \left(\frac{p_{T}+c_{1}}{c_{2}}\right),
$$

where $c_{n}$ are the fit parameters determined for each data subsample taken at different periods of time. Examples of the muon $\epsilon_{L T}^{\ell}$ efficiency parameterization and the fit uncertainties are shown in Fig. 5.2.

\subsection{Simulated Sample Corrections}

Physics analyses at high energy colliders are statistical in nature where millions of simulated events are produced in order to understand the behaviors of different physics processes created by the collisions. Generated MC events of each simulated process are given a primary weight, $w=\frac{(\sigma \times \mathcal{B}) \times \mathcal{L}_{\text {int }}}{N_{\text {tot }}}$, where $\sigma$ and $\mathcal{B}$ are the production cross section and 

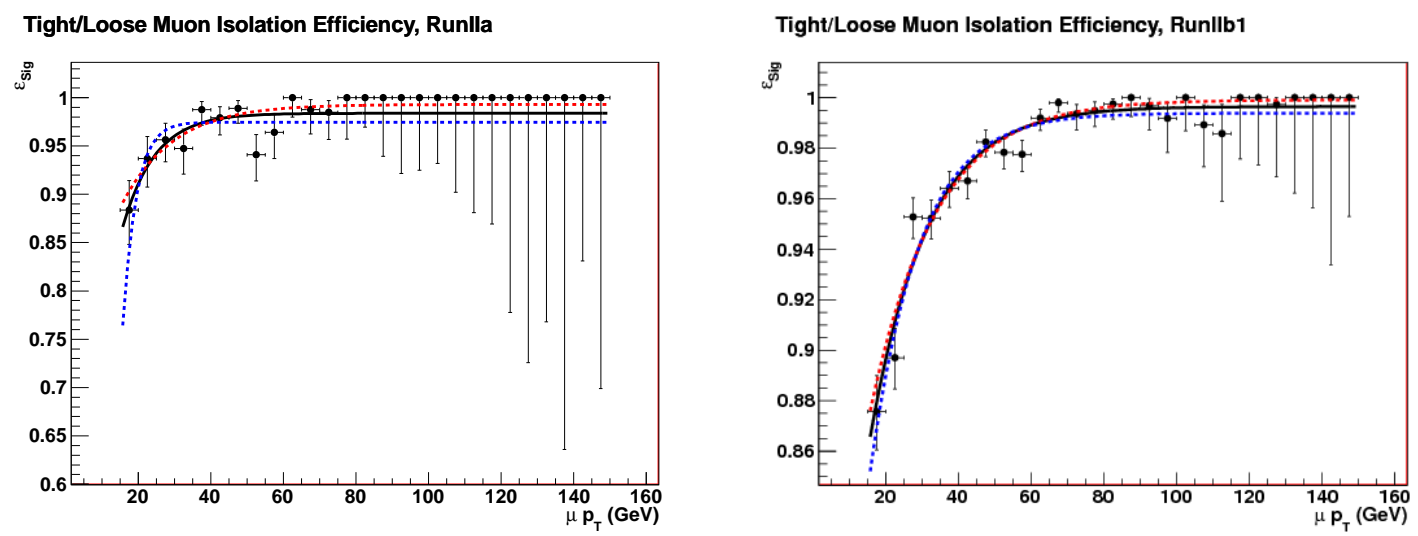

Figure 5.2: Muon efficiency $\epsilon_{L T}^{\ell}$ parametrization as a function of muon $p_{T}$. The red and blue lines are the fit uncertainties.

branching ratio of the process, $\mathcal{L}_{\text {int }}$ is the integrated luminosity used in the analysis, and $N_{\text {tot }}$ is the total number of generated events in each MC samples.

Besides physics motivated corrections, which are applied to MC samples to account for higher-order calculations of cross sections as described above, we also consider another class of corrections used to compensate for possible higher order corrections to specific physics final states and detector modeling variations in the simulations. Processes of deriving corrections for these modeling effects are commonly called reweighting. The corrections coming from reweighting processes are derived in form of scale factors which can be constant or have a kinematic dependency. MC samples are then corrected through scaling the weight of simulated events accordingly.

Most corrections related to detector effects are integrated into the D $\varnothing$ analysis framework, and they are applied in our analyses. To further improve the data modeling and mitigate discrepancies in kinematic distributions, we derive an additional set of corrections based on specific samples and selection criteria to further correct the $V+$ jets $(W+$ jets and $Z+$ jets) sample, the largest background in our analyses. Brief descriptions of common corrections implemented in the $\mathrm{D} \emptyset$ framework and details of our own $V+$ jets reweighting process are given in the following sections.

\subsubsection{Reconstruction Efficiency Corrections}

\section{Lepton Reconstruction Efficiency Corrections}

Scale factors are applied to MC events to account for differences in reconstruction efficiencies between the data and simulation for leptons and jets. For the MC electrons, a scale factor correcting for electron identification (ID) efficiency is parameterized and applied as a function of detector $\eta$, detector $\phi$ and instantaneous luminosity [62]. Similarly, multiple scale factors have been parameterized as functions of detector $\eta$ and $\phi$ and applied to MC 
muons to correct for muon identification efficiency, muon track reconstruction efficiency and muon isolation efficiency [89].

The electron energy scale correction parameterized in detector azimuthal angle $\phi$, physics $\eta$ of the electron and kinematic variables describing shower shape is applied to both data and MC samples. Subsequently, the difference in electron energy resolution of data and $\mathrm{MC}$, which is caused by imperfect descriptions of the material in front of the calorimeter and approximations in the shower development used in the DØ simulation programs, is corrected by electron energy smearing process [90]. The resolution of muon momentum is also not described perfectly in MC. Therefore, the muon transverse momenta reconstructed in $\mathrm{MC}$ are smeared to bring MC into a better agreement with data [91].

\section{Jet Reconstruction Efficiency Corrections}

To correct on average the jet energy of jets measured in the calorimeter to the corresponding particle jets and for the differences in jet energy resolution predicted in $\mathrm{MC}$ and observed in data, JES and JSSR processes described in Chapter 4 are applied to MC samples accordingly. The efficiency of vertex confirmation for jets and the efficiency for a jet to be taggable are also corrected in simulated samples to reproduce the respective efficiencies in data. Both vertex confirmation and taggability scale factors are parameterized as a function of jet $p_{T}$, jet $\eta$, and the Z-coordinate of the primary vertex [92].

Since performance of the $b$-tagging algorithm is different in data and simulated samples, the tagging rate function (TRF) in $\mathrm{MC}$ is not the same as that in data [70]. To correct for this effect, the $b$-jet identification scale factor $\left(\mathrm{SF}_{b}^{i}\right)$ for jets to pass a specific b-tagging working point $(i)$ is applied to each corresponding $b$-tagged data sample.

\subsubsection{Trigger Correction}

The effect of trigger selection is simulated in the MC sample by applying the trigger efficiency, measured in data, as an event weight to each MC event. The trigger efficiency used in our analyses is parametrized as a function of electron or muon $\eta$, azimuthal angle, and transverse momentum $p_{T}$. More details about trigger selection and trigger efficiency will be presented in Chapter 6 .

\subsubsection{Luminosity Reweighting}

In order to provide a realistic simulation of the detector response to beam conditions during actual data collection, an overlay of zero bias data collected using zero-bias triggers is used to define the baseline detector response for each MC event. However, the instantaneous luminosity for the zero-bias overlay does not match the luminosity profile of the dataset under analysis. Therefore, a luminosity reweighting is apply to match the instantaneous luminosity profile in MC to the luminosity profile of the data. 


\subsubsection{Primary Vertex Reweighting}

Due to the emittance growth of the colliding beams, the width of the distribution of the primary vertex longitudinal position $V_{z}$ becomes broader later in the store. The width of $V_{z}$ measured in data depends on the intensity profile of the proton and antiproton beams, the amplitude and oscillation of the transverse motion of bunches, while the $V_{z}$ distribution used in simulating MC events is based on a Gaussian of a fixed width. Therefore, a primary vertex reweighting is used to correct the generic primary vertex distribution in $\mathrm{MC}$ to the distribution measured in data.

\subsection{5 $Z_{p_{T}}$ and $W_{p_{T}}$ Reweighting}

Due to the softscale of radiation emission back-to-back with the vector boson, the $\mathrm{Z}$ boson $p_{T}$ and $\mathrm{W}$ boson $p_{T}$ distributions are poorly modeled by both PYTHIA and ALPGEN for events with $p_{T}^{Z}$ or $p_{T}^{W}$ less than $30 \mathrm{GeV}$. The transverse momentum spectrum of the $\mathrm{Z}$ boson is corrected in the $\mathrm{MC}$ to match that observed in data by reweighting the $Z_{p_{T}}$ distribution. The correction is derived from the $p_{T}^{Z}$ distribution at the generator level and the observed spectrum in the unfolded data [93]. The $p_{T}^{Z}$ reweighting scale factor is applied to simulated events as a function of $p_{T}^{Z}$ in each bin of jet multiplicity. The $\mathrm{W}$ boson $p_{T}$ distribution is also corrected using the same dependence, taking into account differences between the $p_{T}$ spectra of $\mathrm{Z}$ andW bosons predicted in NNLO QCD [94, 95].

\subsection{6 $V+$ jets Reweighting}

After all standard corrections for physics modeling and detector effects implemented in the $\mathrm{D} \varnothing$ framework have been applied to preselected samples in our analyses, the kinematic distributions in data and simulated events show remaining residual differences in several kinematic distributions. Motivated by comparisons of MC events simulaed with ALPGEN and data [96], and MC samples simulated with other event generators [76], we develop corrections to $W+$ jets and $Z+$ jets MC samples to correct for the shape discrepancies in kinematic distributions between data and simulation. The corrections are derived based on the direct comparison between selected data and $\mathrm{MC}$ events passing our preselection criteria (before applying $b$-tagging), where any contamination from signal is very small.

To improve the description of the jet directions, we correct the $\eta$ distributions of the leading and second leading jets ( jets with the highest and second highest transverse momentum) in $V+$ jets events. The lepton $\eta$ distribution in $W+$ jets $(Z+$ jets $)$ MC events in the $\ell \nu j j(Z H \rightarrow \ell \ell b b)$ analysis is also corrected to reproduce the distribution of final state leptons associated with $W / Z$ decays in data. Correlated discrepancies observed in distributions of transverse momentum $p_{T}^{W}$ of the leptonically decaying $W$ boson and the jet angular

separation between two leading jets, $\Delta \mathcal{R}\left(j_{1}, j_{2}\right)=\sqrt{\left(\eta_{\text {jet } 1}-\eta_{\text {jet } 2}\right)^{2}+\left(\phi_{\text {jet } 1}-\phi^{j 2}\right)^{2}}$, are corrected simultaneously in the two-dimensional $\left(\Delta \mathcal{R}\left(j_{1}, j_{2}\right), p_{T}^{W}\right)$ plane [97]. 

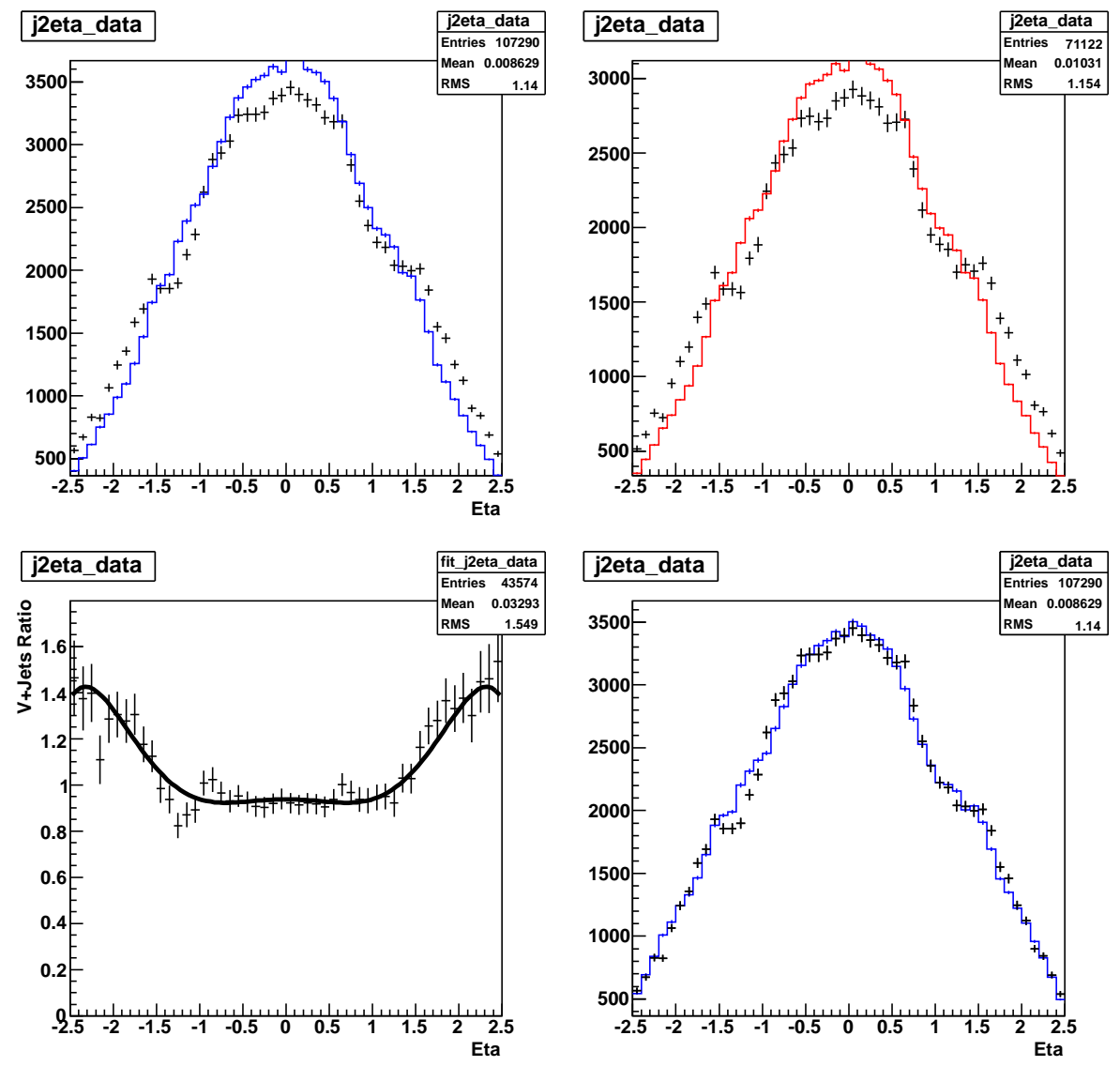

Figure 5.3: Performance of the second leading jet $\eta$ reweighting. The top-left plot shows data (points) and all backgrounds (blue) before reweighting; the top-right plot shows the non- $V+$ jets background-subtracted data (points) and $V+$ jets sample (red); the bottomleft plot shows the fit to the ratio of non- $V+$ jets background-subtracted data and $V+$ jets background; the bottom-right plot shows data (points) and all backgrounds (blue) after applying the second jet reweighting.

\section{Jet $\eta$ Reweighting}

Due to the deficiencies in the MC programs and the parameters that are used in the event generation, the jet $\eta$ distributions of the $V+$ jets samples generated by ALPGEN interfaced with PYTHIA do not agree with our data. In particular, the $\eta$-distributions observed in data are broader than in simulation. To compensate for the observed deviations we apply reweighting scale factors to each generated event in the $W+$ jets and $Z+$ jets MC samples to better match the observed data. Two reweighting processes are applied in succession, the first one is for the $\eta$ distribution of the second leading jet $\left(\eta_{\text {jet } 2}\right)$ followed by another for the $\eta$ ditribution of the leading jet $\left(\eta_{j e t 1}\right)$.

The jet $\eta$ reweighting procedure starts with normalizing the total number of $W+$ jets and $Z+$ jets $\mathrm{MC}$ events to the number of events in the $V+$ jets component of data $\left(N_{V+\text { jets }}^{\text {data }}\right)$, 
which is is obtained by subtracting the MJ background $\left(N_{M J}\right)$ and other non- $W+$ jets or non- $Z+$ jets SM background sources $\left(N_{\text {nonV jets }}^{M C}\right)$ from the selected data sample $N_{\text {data }}$ :

$$
N_{V+j e t s}^{\text {data }}=N_{\text {data }}-N_{M J}-N_{\text {nonV jets }}^{M C}
$$

The jet $\eta$ reweighting scale factor is then parameterized as function of jet $\eta$ based on the ratio of jet $\eta$ distributions between simulated $V+$ jets sample and data after subtraction of the none $V+$ jets backgrounds and :

$$
f(\eta)=p_{0}+p_{1} \times \eta^{2}+p_{2} \times \eta^{4}+p_{2} \times \eta^{4} .
$$

The normalization of the $\mathrm{V}+$ jets sample is constrained during the fit for the parameters $p_{i}$ of the reweighting function (Eqn. (5.15)). The agreement in the distribution of $\eta_{\text {jet } 2}$ between data and $\mathrm{MC}$ before and after applying $\eta_{\text {jet } 2}$ reweighting along with the fit based on preselected samples of $\ell \nu j j$ analysis are show in Fig. 5.3 .

\section{Lepton $\eta$ Reweighting}

To account for mismodeling of lepton directions in the $\ell \nu j j$ analysis, we reweight the $W+$ jets samples using a second-order polynomial reweighting function of lepton $\eta\left(\eta_{\text {lepton }}\right)$ :

$$
f\left(\eta_{\text {lepton }}\right)=p_{0}+p_{1} \times \eta_{\text {lepton }}^{2},
$$

which is extracted from the ratio of lepton $\eta$ distributions between simulated $W+$ jets sample and data after subtracting QCD and the other non- $W+$ jets physics background. Similar to the jet $\eta$ fitting procedure, the $W+$ jets sample is first normalized to the corresponding background-subtracted data, and the normalization is constrained during the fitting. The lepton $\eta$ reweigting is also applied in the $Z H \rightarrow \ell \ell b b$ analysis where the distribution of leading $p_{T}$ lepton in the $Z+$ jets sample is corrected to that of background-subtracted data.

\section{$\Delta \mathcal{R}(j 1, j 2)$ and $p_{T}^{W}$ Reweighting}

To account for residual discrepancies in modeling the $p_{T}^{W}$ and the separation of the two leading jets $\Delta \mathcal{R}(j 1, j 2)$ as well as for strong correlation between these two kinematic variables, we perform a simultaneous fit in both $p_{T}^{W}$ and $\Delta \mathcal{R}(j 1, j 2)$ using the two dimensional reweighting function $f_{p_{T}^{W}} \times f_{\Delta \mathcal{R}}$, where:

$$
\begin{aligned}
& f_{p_{T}^{W}}=a_{0}+a_{1} \times\left[1-\operatorname{erf}\left(\frac{p_{T}^{W}-a_{2}}{a_{3}}\right)\right]+a_{4} \times \exp \left(\frac{-0.5 \times\left(p_{T}^{W}\right)^{4}}{a_{5}^{2}}\right), \\
& f_{\Delta \mathcal{R}}=b_{0}+b_{1} \times \Delta \mathcal{R}+b_{2} \times(\Delta \mathcal{R})^{2}+c_{3} \times(\Delta \mathcal{R})^{3} .
\end{aligned}
$$

The $a_{i}$ and $b_{i}$ parameters in the pair of $f_{p_{T}^{W}}$ and $f_{\Delta \mathcal{R}}$ reweighting functions are determined from the $2 \mathrm{D}$ fit in the $\left(\Delta \mathcal{R}\left(j_{1}, j_{2}\right), p_{T}^{W}\right)$ plane to the ratios of $W+$ jets MC sample to the 
data/wjet

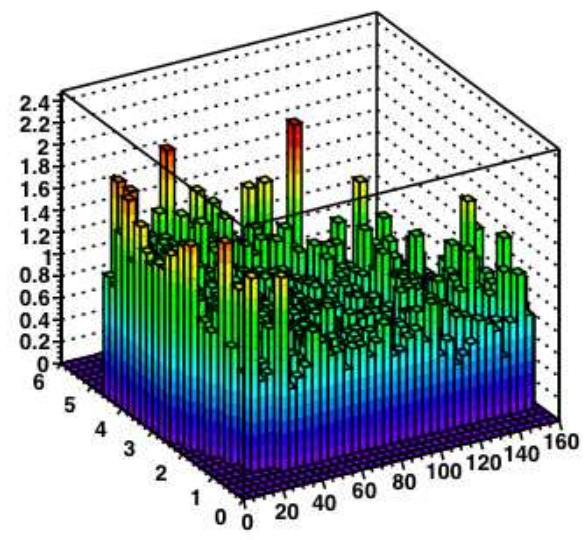

2dfit

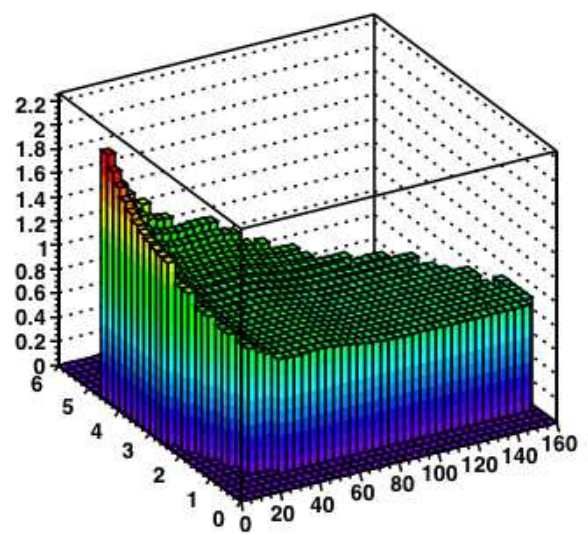

Figure 5.4: The 2D reweighting in the $\left(p_{T}^{W}, \Delta \mathcal{R}(j 1, j 2)\right)$ plane: (left) Ratio of backgroundsubtracted data (points) and simulated $W+$ jets, (right) $2 \mathrm{D}$ fitting function $f_{p_{T}^{W}} \times f_{\Delta \mathcal{R}}$

non- $V+$ jets component of the data, which is obtained by subtracting the MJ background and other non- $W+$ jets SM background sources from the data sample (see Fig. 5.4). The $p_{T}^{W}$ reweighting is applied only to $W+j$ ets events, while the $\Delta \mathcal{R}(j 1, j 2)$ reweighting is applied to both $W+$ jets and $Z+$ jets events.

Reweighting functions from each $V+$ jets reweighting process are shown in Fig. 5.5. The corrections derived from each $V+$ jets reweighting procedure is designed to change differential distributions, but to preserve normalization. The distributions of $\eta_{\text {jet } 2}, \eta_{\text {jet } 1}$, $p_{T}^{W}$, and $\Delta \mathcal{R}(j 1, j 2)$ in data and MC before and after appying all corrections from $V+$ jets reweighting procedures are shown in Fig. 5.6. These corrections are on the order of a few percent in the highly populated region of each distribution and may exceed $10 \%$ in the extreme values of each distribution. 

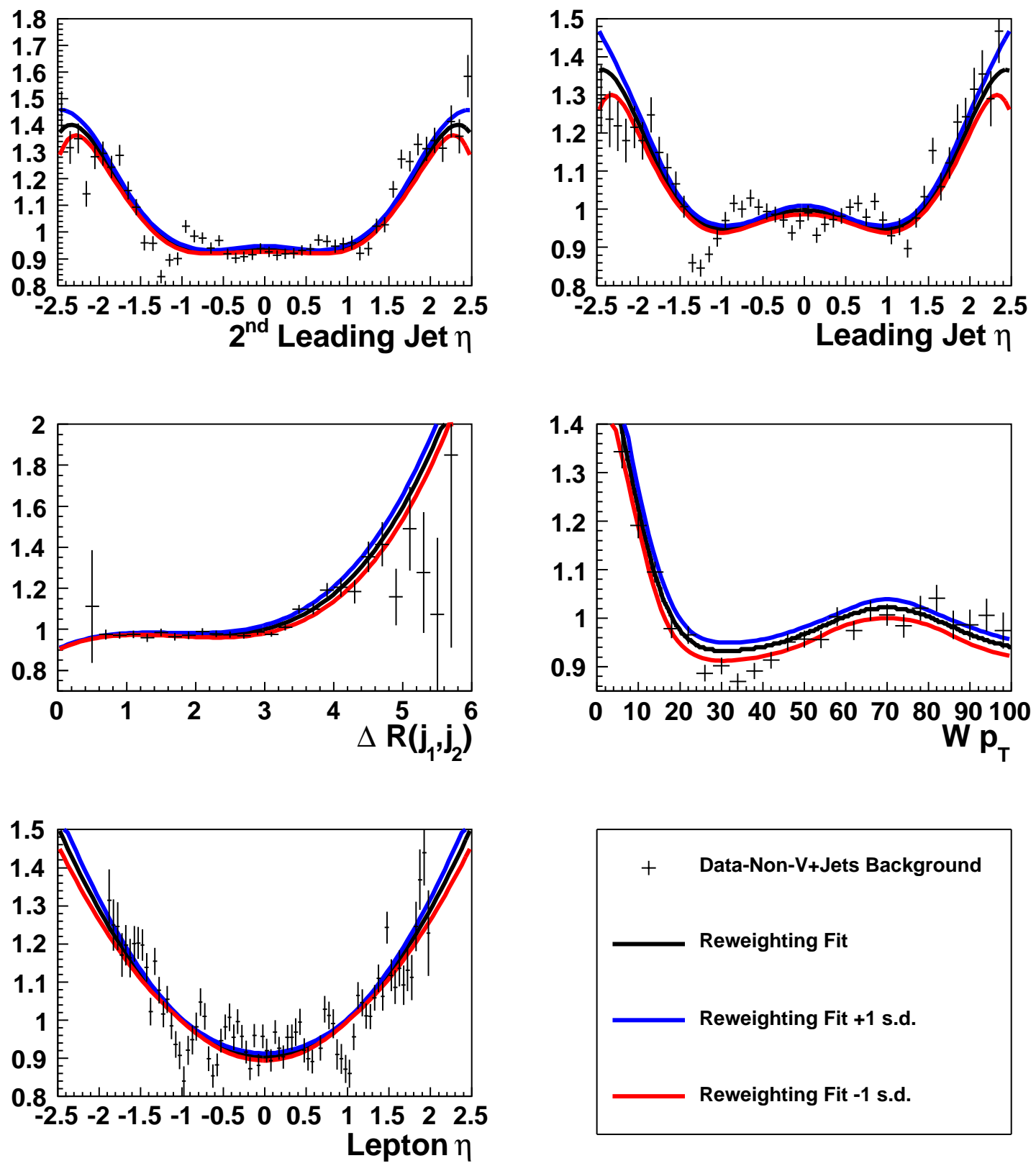

Figure 5.5: Reweighting fuctions applied to correct $\eta_{\text {jet } 2}$ (top left), $\eta_{\text {jet } 2}$ (top right), $\eta_{\text {lepton }}$ (bottom) and simultaneously correct for $p_{T}^{W}$ and $\Delta \mathcal{R}(j 1, j 2)$ ) (middle). In each plot, the black curve represents the nominal reweighting function, while the red and blue curves represent $\pm 1 \sigma$ variations on the fit function. 

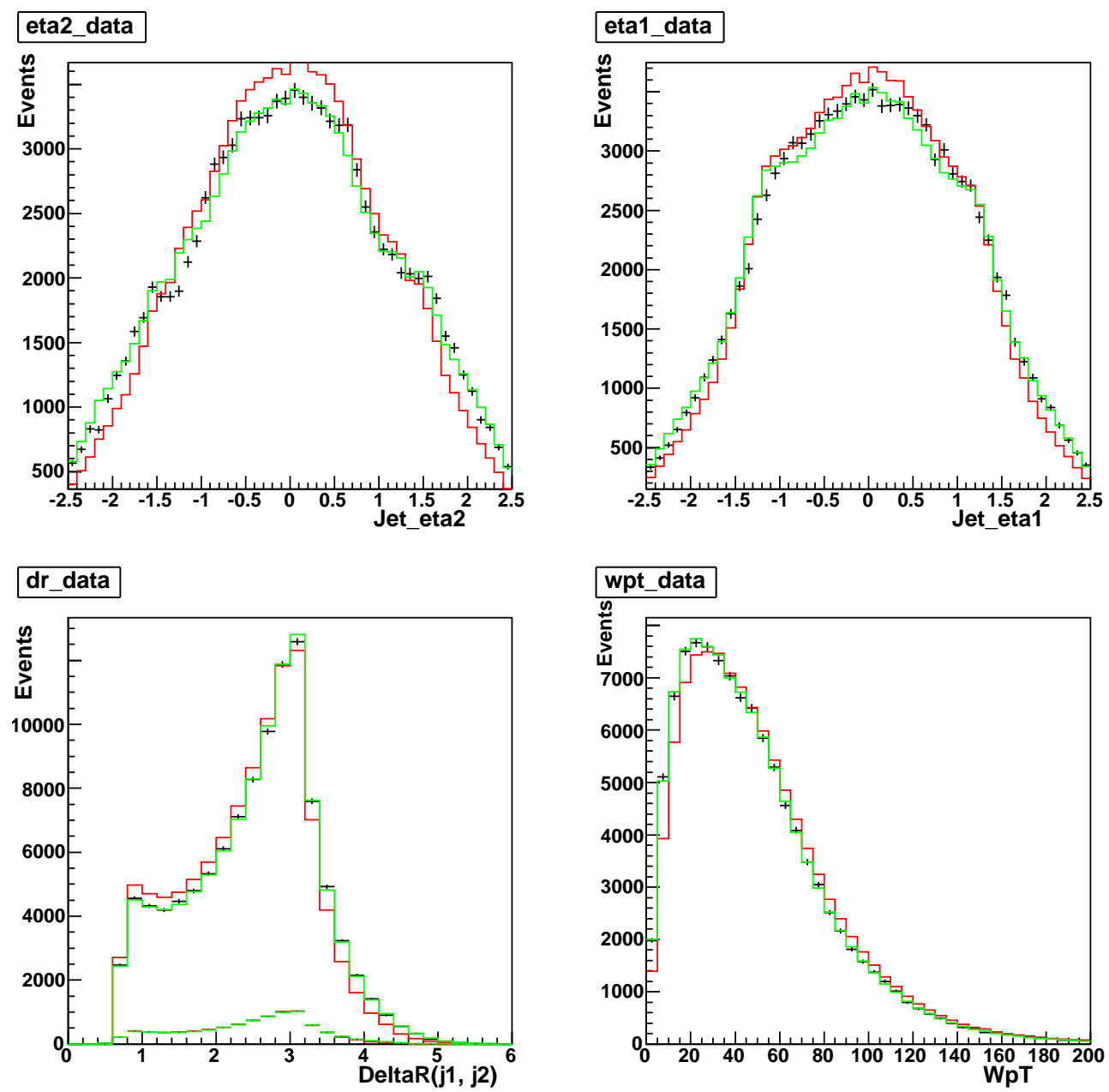

Figure 5.6: Distributions of $\eta_{j e t 2}, \eta_{j e t 1}, p_{T}^{W}$, and $\Delta \mathcal{R}(j 1, j 2)$ in data (black) and MC before (red) and after (green) appying all corrections from $V+$ jets reweighting. 
6

\section{Event Selections}

Event selection in both $\ell \nu j j$ and $Z H \rightarrow \ell \ell b b$ analyses is based on signal signatures and kinematics. The $\ell \nu j j$ analysis requires topologies with an isolated charged lepton and a significant imbalance in the transverse energy, which indicates products of $W \rightarrow e \nu$ or $W \rightarrow \mu \nu$ decays, and at least two jets. The $Z H \rightarrow \ell \ell b b$ analysis requires two isolated charged leptons to reconstruct the $Z$ candidate decaying as $Z \rightarrow e^{+} e^{-}$or $Z \rightarrow \mu^{+} \mu^{-}$and at least two jets. The selection criteria in each analysis are designed to maximize the acceptance of the considered SM Higgs boson signals and to suppress the background as much as possible, while maintaining good data-to-MC agreement in the preselected sample.

Taking into account the differences in background composition and signal topologies, the search in each analysis is then organized into sub-channels according to various lepton, jet multiplicity, and $b$-tagging characterization criteria in order to optimize the overall signal sensitivity. The event selection proceeds in two steps, online event selection (event triggering) and offline event selection. Both selection steps along with the channel subdivisions in each of $\ell \nu j j$ and $Z H \rightarrow \ell \ell b b$ analyses are described in this chapter.

\subsection{Event Selection in $\ell \nu j j$ Analysis}

\subsubsection{Online Event Selection}

\section{Event triggering in electron channel $(e \nu j j)$}

Events in the electron channel have the final state containing an electron candidate expected from $W \rightarrow \ell \nu$ decays. These are triggered by a logical OR of Single-Lepton and Lepton+Jets triggers requiring a candidate electromagnetic object and jets [98]. The trigger efficiency is parameterized as a function of the transverse momentum $p_{T}$ and and $\eta$ of the leading electron candidate in the event, and it is applied as an event weight to simulated samples. For the events selected in our analyses, these triggers have a typically efficiency of above $90 \%$. 


\section{Event triggering in muon channel $(\mu \nu j j)$}

For the muon channel, we accept events fired by any available trigger, except those containing lifetime-based requirements that can bias the performance of $b$-jet identification. The efficiency of this inclusive set of triggers is obtained by applying a trigger correction, $P_{\text {corr }}$, to the efficiency of a well-modeled logical OR of the Single-Muon and Muon+Jets triggers $\left(T_{\mu \mathrm{OR}}\right)$. The correction is derived from comparing data events selected with $T_{\mu \mathrm{OR}}$ triggers to events selected using all triggers:

$$
P_{\text {corr }}=\frac{\left(N_{\text {Data }}-N_{M J}\right)_{i n c l}-\left(N_{\text {Data }}-N_{M J}\right)_{T_{\mu \mathrm{OR}}}}{N_{M C}},
$$

where the numerator is the difference between the number of data events in the inclusive trigger sample and the $T_{\mu \mathrm{OR}}$ trigger sample, after subtracting off the multijet (MJ) backgrounds, and the denominator is the number of MC events with the trigger efficiency set to 1. The inclusive trigger efficiency estimate for events in the muon channel is $T_{\mu \mathrm{OR}}+P_{\text {corr }}$ (limited to be $\leq 1$ ).

Using the inclusive set of triggers results in a gain of about $30 \%$ in efficiency over using only Single-Muon and the Muon+Jets triggers $\left(T_{\mu \mathrm{OR}} \sim 70 \%\right)$. The most significant contribution to this improvement comes from triggers based on jets and $E_{\mathrm{T}}$. To account for the differences in the contribution and the efficiency of different triggers, the inclusive trigger correction $P_{\text {corr }}$ is parametrized as a function of the scalar sum of the transverse momenta of all jets, $H_{T}$, and the $\mathbb{E}_{\mathrm{T}}$ for separate regions in muon $\eta$.
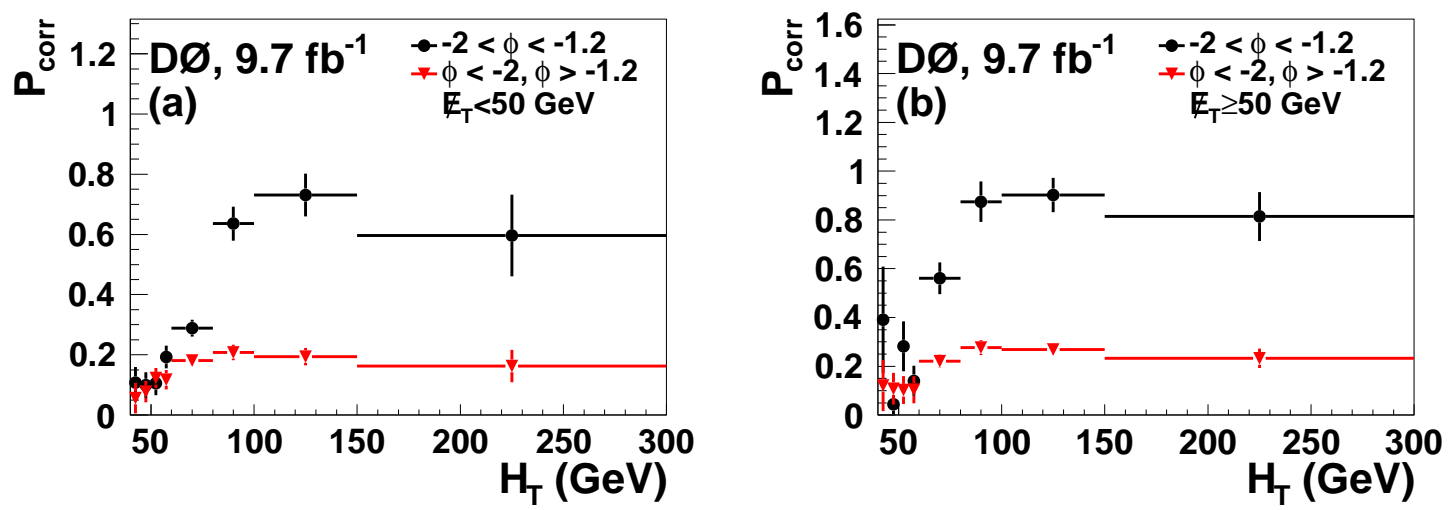

Figure 6.1: Data-derived muon trigger correction to account for the resulting efficiency gain in moving from single muon and muon+jets triggers to inclusive triggers as a function of $H_{T}$ for $|\eta|<1.0$, shown (a) for events with $\mathrm{E}_{\mathrm{T}}<50 \mathrm{GeV}$ and (b) for events with $\mathrm{E}_{\mathrm{T}}<50 \mathrm{GeV}$. The black circles show the correction when the muon is in the region of $\phi(-2<\phi<-1.2)$, where the detector support structure allows only partial coverage by the muon system, and the red triangles show the correction elsewhere in $\phi$. 


\subsubsection{Offline Event Selection}

The preselection of events for the $e \nu j j$ or $\mu \nu j j$ channel includes events satisfying a set of selection criteria based on the identification and kinematics of physics objects in the final state. The selection cuts applied for each channel are discussed in the following sections.

\section{- Primary vertex selection}

The event selection in both electron and muon channels requires a $p \bar{p}$ interaction vertex that has at least three associated tracks, and is located within $60 \mathrm{~cm}$ of the center of the detector along the beam direction $\left(P V_{z} \leq 60 \mathrm{~cm}\right)$. If an event has more than one such vertex, the vertex with the highest average $p_{T}$-value of its associated tracks is chosen. This requirement ensures that selected events are from collisions occurring in the central part of the detector, where the activity from the primary interaction can be covered by the SMT and physics objects in event final state are more likely to be within the detector acceptance.

\section{- Lepton selection}

The requirement of the final state to contain exactly one isolated electron or muon candidate coming from the leptonic decay of a $W$ boson significantly suppresses the multijet background and that from $Z(\rightarrow \ell \ell)+$ jets and $t \bar{t}$ events. Two sets of lepton identification criteria are applied for each lepton channel in order to form a "tight" sample used to perform the search for the SM Higgs boson and a "loose" sample used to estimate the multijet background as described in Sec. 5.5.

For the electron channel, events having a single electron with transverse momentum $p_{T}>15 \mathrm{GeV}$ within a pseudorapidity range of $|\eta|<1.1$ (CC) or $1.5<|\eta|<2.5$ (EC) are selected. We exclude the gap situated between the central and the end-cap calorimeter in the pseudorapidity range of $1.1<|\eta|<1.5$. The electrons passing the kinematic selections above are further required to satisfy identification criteria, which are optimized separately for the $\mathrm{CC}$ and EC regions. The two sets of identification criteria used to select tight and loose electron samples for each calorimeter region are listed in Table 4.2.1 (Sec. 4.2.1).

For the muon channel, we select events having exactly one muon with $p_{T}>15 \mathrm{GeV}$ and $|\eta|<2.0$. Muon candidates are then required to fulfill identification criteria related to the muon track quality and the isolation of muon from particles originating from hadron decay products, which are defined in Sec. 4.2.2. Selected events in the loose or tight samples must contain a muon candidate with a medium quality local track identified in the muon system matched to a medium quality central track. The muons in the tight and loose samples are required to satisfy the tight and loose isolation criteria, respectively.

\section{- Missing transverse energy selection}


To reconstruct the candidate $W(\rightarrow \ell \nu)$ boson, selected events are required to contain a significant $\mathrm{E}_{\mathrm{T}}$, which characterizes the presence of the neutrino. The missing transverse energy is reconstructed as described in Sec. 4.2.4, and is required to satisfy $E_{\mathrm{T}}>15 \mathrm{GeV}$ for the electron channel and $\mathrm{E}_{\mathrm{T}}>20 \mathrm{GeV}$ for the muon channel. The $\mathrm{E}_{\mathrm{T}}$ cut is efficient in both removing mismodeled events and reducing the multijet dominated region at low $\mathrm{E}_{\mathrm{T}}^{\mathrm{T}}$.

\section{- Jet selection}

Events considered in $\ell \nu j j$ analyses are required to contain at least two jets originating from the primary $p \bar{p}$ vertex. Besides satisfying the identification criteria described in Sec. 4.2.3, these jets must have $p_{T}>20 \mathrm{GeV}$ and within $|\eta|<2.5$.

To group events with similar signal-to-background ratio and optimize the search for specific signal processes, the preselected sample of each lepton channel is then split into three orthogonal subsamples based on jet multiplicity: two jet exclusive, three jet exclusive and four jet inclusive. The dominant signal contribution in the twojet exclusive and three-jet exclusive channels comes from the $W H \rightarrow \ell \nu b \bar{b}$ and $H \rightarrow$ $W W^{*} \rightarrow \ell \nu j j$ processes, while the majority of signal events in the four-jet inclusive channel is from the $V H \rightarrow V W W^{*} \rightarrow \ell \nu j j j j$ process.

\section{- Mutijet background veto}

The transverse mass $M_{T}^{W}$ of the leptonically decaying $W(\rightarrow \ell \nu)$ boson is reconstructed from the $\left(\ell, E_{T}\right)$ system using missing transverse energy, the lepton transverse energy $\left(E_{T}^{\ell}\right)$, and the azimuthal separation $\Delta \phi\left(\ell, E_{T}\right)$ between the isolated lepton and the $E_{T}$ vector:

$$
\left(M_{T}^{W}\right)^{2} \equiv 2 p_{T}^{\ell} \mathbb{E}_{T}\left[1-\cos \Delta \phi\left(\ell, E_{T}\right)\right]
$$

Multijet events contribute a significant rate at small values of $M_{T}^{W}$ and the signal-tobackground ratio is rather small at low $\mathbb{E}_{T}$ region $[99,98]$. Therefore, we apply a triangular cut on the $M_{T}^{W}$ :

$$
M_{T}^{W}>40 \mathrm{GeV}-0.5 \times E_{T}
$$

in order to suppress overwhelming background from multijet events (this is developed using control samples in the data as described in Sec. 5.5).

\subsubsection{Normalization at the Preselection}

After applying the MC corrections described in Sec. 5.6 to simulated events, we adjust the normalization of the $V+$ jets and MJ backgrounds to bring the total number of predicted background events in agreement with data. The normalization factors applied to $V+$ jets and MJ backgrounds are determined via a simultaneous template fit of the $M_{T}^{W}$ distribution to the $V+$ jets and MJ component of data, which is obtained by subtracting non- $V+$ jet 
simulated background sources from the preselected data sample. We perform separate fits for each lepton flavor and jet multiplicity category. Figure 6.2 shows examples of the simultaneous fits for two and three jet exclusive samples in muon channel. The $V+$ jets experimental normalization factors for the two jet and three channels are 1.02 and 1.24, respectively. The distribution of $M_{T}^{W}$ after this normalization procedure along with the distributions of muon $p_{T}$ and azimuthal angle between the muon and neutrino for the muon channel are shown in Fig. 6.3. Distributions of the transverse momentum of the two jets with the highest $p_{T}$ and the dijet mass reconstructed from them show good agreement between data and combined background for preselected events in the muon channel (see Fig. 6.4).
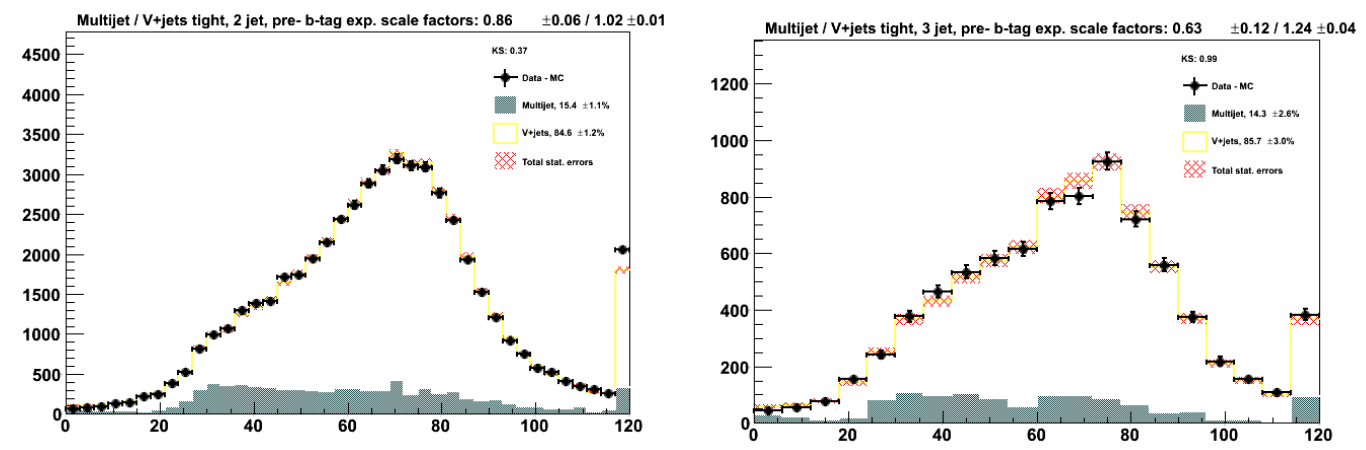

Figure 6.2: Simultaneous fit of the $V+$ jets and MJ backgrounds in the $W(\rightarrow \ell \nu)$ transverse mass $\left(M_{T}^{W}\right)$ distribution, in the muon two jet (left) and muon three jet (right) channels. The $V+$ jets experimental normalization factors for the two jet and three channels are 1.02 and 1.24 , respectively.

\subsubsection{Classifying $b$-tag Samples}

To focus on the number and quality of identified $\mathrm{b}$ jets in preselected events, we define six independent tagging samples based on the $b$-jet identification discriminant output $\left(b_{I D}\right)$ described in Sec. 4.2.5. The criteria for categorizing $b$-tag samples are as follows:

- a zero $b$-tag (0Tag) sample includes events with no jet satisfying the $b$-tagging criteria;

- a one-loose $b$-tag (1L) sample includes events with exactly one $b$-tagged jet that passes the loose selection requirement $\left(b_{I D}>0.02\right)$ but fails the tight selection requirement $\left(b_{I D}>0.15\right)$

- a one-tight $b$-tag (1T) sample includes events having exactly one $b$-tagged jet that has a $b$-identification discriminant output satisfying the tight $b$-jet selection threshold $\left(b_{I D}>0.15\right)$

- a two-loose $b$-tag (2L) sample includes events with two or more $b$-tagged jets that have an average value of the $b$-identification discriminant of the two leading $b$-tagged jets in the range $0.02<\left(b_{I D}^{j 1}+b_{I D}^{j 1}\right) / 2 \leq 0.35$; 

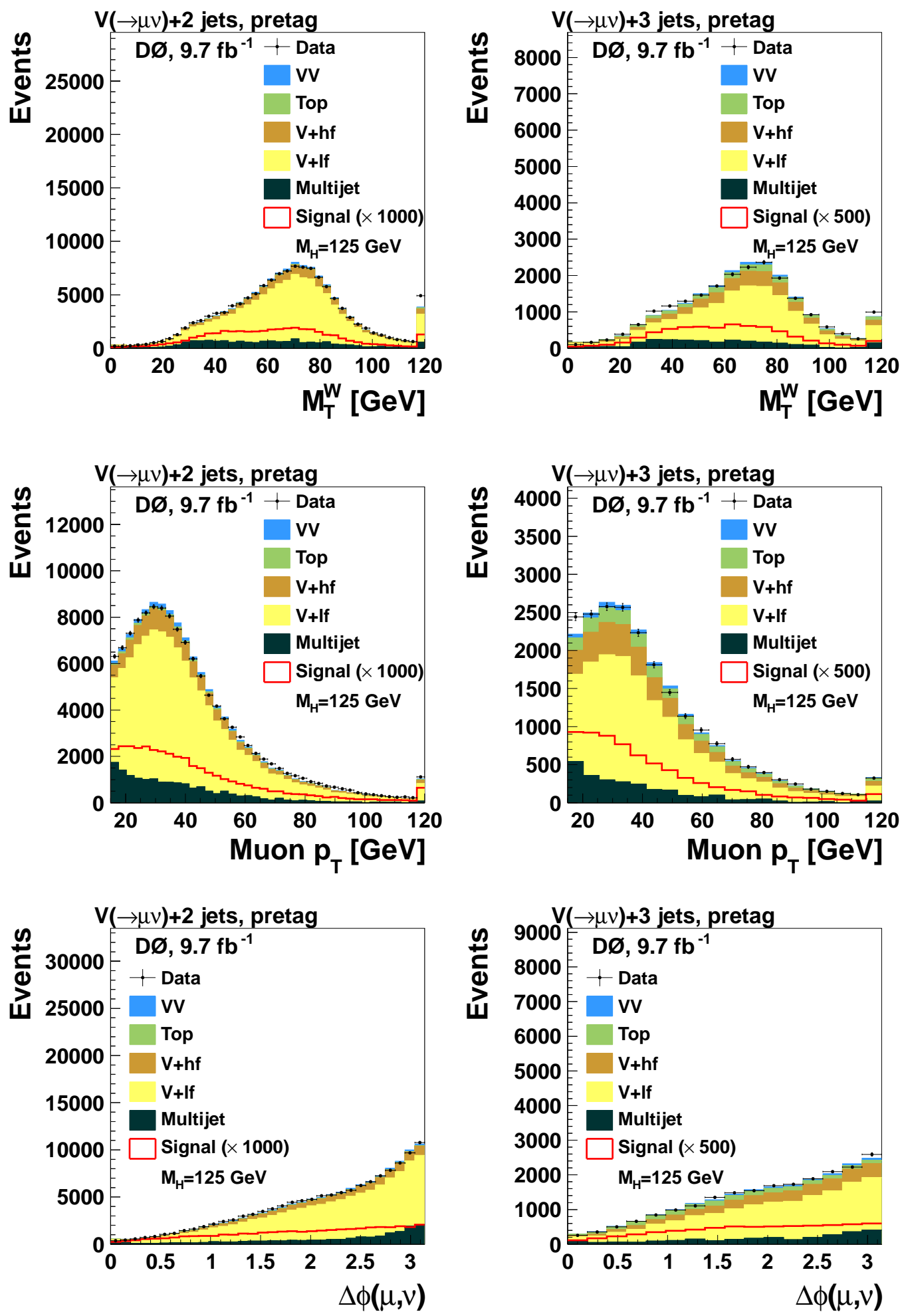

Figure 6.3: Kinematic distributions related to the lepton and $\mathrm{E}_{\mathrm{T}}$ for the two jet (left) and three jet (right) event samples in the muon channel after preselection. 

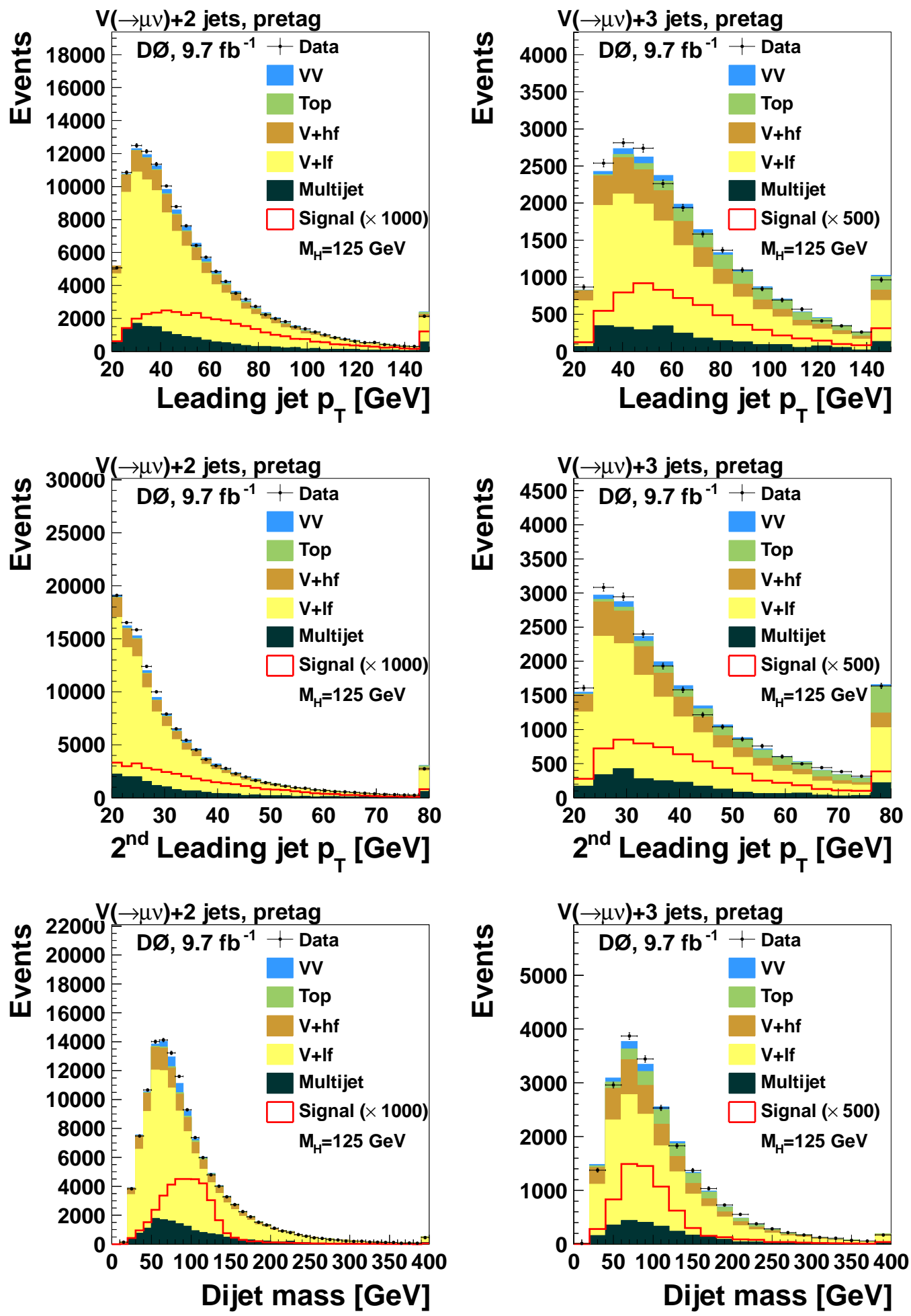

Figure 6.4: Kinematic distributions related to the leading jets for the two jet (left) and three jet (right) event samples in the muon channel after preselection. 
- a two-medium $b$-tag (2M) sample includes events with two or more $b$-tagged jets that satisfy the requirement $0.35<\left(b_{I D}^{j 1}+b_{I D}^{j 2}\right) / 2 \leq 0.55$;

- a two-tight $b$-tag $(2 \mathrm{~T})$ sample includes events with two or more $b$-tagged jets that satisfy the requirement $0.55<\left(b_{I D}^{j 1}+b_{I D}^{j 2}\right) / 2$.

The expected number of events from each signal and background category is compared to the data for each $b$-jet identification category for events in the muon channel with two (three) jets in Table 6.1 (Table 6.2). The signal yield and expected background for both lepton samples combined for the two jets, three jets, and four or more jets are shown in Tables 6.3, 6.4, and 6.5. The significant change in signal-to-background ratio from the lower $b$-tag categories to the higher $b$-tag categories can be visualized from the distribution of dijet invariant mass for events with two jets in all $b$-tag categories shown in Fig. 6.5. In all plots, data points are shown with error bars that reflect the statistical uncertainty only. Discrepancies in data-MC are within our systematic uncertainties described in Sec. 8.1.

\begin{tabular}{lrrrrrrr}
\hline \hline & Pretag & 0Tag & $1 \mathrm{~L}$ & $1 \mathrm{~T}$ & $2 \mathrm{~L}$ & $2 \mathrm{M}$ & $2 \mathrm{~T}$ \\
\hline$V H \rightarrow \ell \nu b \bar{b}$ & $18.20(0.000)$ & 3.08 & 1.95 & 5.53 & 1.55 & 2.28 & 3.79 \\
$H \rightarrow V V \rightarrow \ell \nu j j$ & $11.61(66.36)$ & 8.80 & 1.81 & 0.84 & 0.15 & 0.04 & 0.00 \\
$V H \rightarrow V V V \rightarrow \ell \nu j j j j$ & $5.87(13.15)$ & 4.24 & 1.03 & 0.50 & 0.12 & 0.01 & 0.00 \\
\hline Diboson & 2809 & 1992 & 477 & 265 & 53 & 21 & 19 \\
$V+(g, u, d, s)$-jets & 92542 & 75726 & 13184 & 3053 & 870 & 68 & 7 \\
$V+(b \bar{b} / c \bar{c})$ & 13846 & 7598 & 2447 & 2640 & 497 & 352 & 349 \\
top $(t \bar{t}+$ single top) & 1595 & 344 & 202 & 586 & 109 & 149 & 210 \\
Multijet & 16015 & 11590 & 2798 & 1293 & 327 & 129 & 91 \\
\hline Total expectation & 126807 & 97250 & 19108 & 7837 & 1856 & 719 & 676 \\
Total uncertainty & \pm 7576 & \pm 4752 & \pm 1164 & \pm 835 & \pm 177 & \pm 87 & \pm 85 \\
Observed events & 126811 & 96613 & 19668 & 7460 & 1870 & 656 & 544 \\
\hline \hline
\end{tabular}

Table 6.1: Observed number of events in data and expected number of events from each signal and background source (where $V=W, Z$ ) for events with exactly two jets in the muon channel. The expected signal is quoted at $M_{H}=125 \mathrm{GeV}$, except for the pretag sample where the expected signal is also quoted (in parentheses) for $M_{H}=165 \mathrm{GeV}$. The total background uncertainty includes all sources of systematic uncertainty added in quadrature. 


\begin{tabular}{lrrrrrrr}
\hline \hline & Pretag & 0Tag & $1 \mathrm{~L}$ & $1 \mathrm{~T}$ & $2 \mathrm{~L}$ & $2 \mathrm{M}$ & $2 \mathrm{~T}$ \\
\hline$V H \rightarrow \ell \nu b b$ & $4.15(0.000)$ & 0.62 & 0.47 & 1.14 & 0.43 & 0.55 & 0.84 \\
$H \rightarrow V V \rightarrow \ell \nu j j$ & $4.29(24.35)$ & 2.95 & 0.84 & 0.39 & 0.13 & 0.03 & 0.00 \\
$V H \rightarrow V V V \rightarrow \ell \nu j j j j$ & $3.36(12.27)$ & 2.12 & 0.78 & 0.37 & 0.13 & 0.02 & 0.00 \\
\hline Diboson & 567 & 361 & 119 & 55 & 21 & 6.4 & 5.1 \\
$V+(g, u, d, s)$-jets & 12969 & 9757 & 2440 & 517 & 313 & 17 & 2.2 \\
$V+(b \bar{b} / c \bar{c})$ & 3502 & 1708 & 706 & 655 & 218 & 121 & 87 \\
top $(t \bar{t}+$ single top) & 1753 & 268 & 197 & 526 & 173 & 220 & 272 \\
Multijet & 2508 & 1466 & 572 & 284 & 105 & 40 & 18 \\
\hline Total expectation & 21299 & 13560 & 4034 & 2037 & 830 & 405 & 385 \\
Total uncertainty & \pm 1360 & \pm 735 & \pm 263 & \pm 205 & \pm 73 & \pm 41 & \pm 40 \\
Observed events & 21297 & 13370 & 4071 & 2027 & 828 & 413 & 401 \\
\hline \hline
\end{tabular}

Table 6.2: Observed number of events in data and expected number of events from each signal and background source (where $V=W, Z$ ) for events with exactly three jets in the muon channel. The expected signal is quoted at $M_{H}=125 \mathrm{GeV}$, except for the pretag sample where the expected signal is also quoted (in parentheses) for $M_{H}=165 \mathrm{GeV}$. The total background uncertainty includes all sources of systematic uncertainty added in quadrature.

\begin{tabular}{|c|c|c|c|c|c|c|c|}
\hline & Pretag & 0Tag & $\overline{1 \mathrm{~L}}$ & 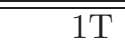 & $\overline{2 \mathrm{LL}}$ & $\overline{\overline{2 M}}$ & $2 \mathrm{~T}$ \\
\hline$V H \rightarrow \ell \nu b b$ & 37.3 & 6.4 & 4.0 & 11.6 & 3.2 & 4.6 & 7.7 \\
\hline$H \rightarrow V V \rightarrow \ell \nu j j$ & 24.7 & 18.8 & 3.9 & 1.8 & 0.3 & 0.07 & 0 \\
\hline$V H \rightarrow V V V \rightarrow \ell \nu j j j j$ & 13.0 & 9.3 & 2.3 & 1.2 & 0.3 & 0.04 & 0.01 \\
\hline Diboson & 5686 & 4035 & 968 & 535 & 109 & 42 & 38 \\
\hline$V+(g, u, d, s)$-jets & 182271 & 148686 & 26421 & 6174 & 1762 & 132 & 13 \\
\hline$V+(b \bar{b} / c \bar{c})$ & 27443 & 15089 & 4872 & 5236 & 978 & 691 & 691 \\
\hline top $(t \bar{t}+$ single top $)$ & 3528 & 758 & 455 & 1289 & 247 & 333 & 462 \\
\hline Multijet & 58002 & 43546 & 9316 & 3700 & 946 & 298 & 195 \\
\hline Total expectation & 276930 & 212114 & 42032 & 16935 & 4043 & 1496 & 1400 \\
\hline Total uncertainty & \pm 14998 & \pm 11352 & \pm 2438 & \pm 1696 & \pm 362 & \pm 117 & \pm 175 \\
\hline Observed events & 276929 & 211169 & 42774 & 16406 & 4057 & 1358 & 1165 \\
\hline
\end{tabular}

Table 6.3: Observed number of events in data and expected number of events from each signal and background source (where $V=W, Z$ ) for events with exactly two jets in muon and electron channels combined. The expected signal is quoted at $M_{H}=125 \mathrm{GeV}$. The total background uncertainty includes all sources of systematic uncertainty added in quadrature. 


\begin{tabular}{lrrrrrrr}
\hline \hline & Pretag & 0 Tag & $1 \mathrm{~L}$ & $1 \mathrm{~T}$ & $2 \mathrm{~L}$ & $2 \mathrm{M}$ & $2 \mathrm{~T}$ \\
\hline$V H \rightarrow \ell \nu b b$ & 8.6 & 1.3 & 1.0 & 2.4 & 0.9 & 1.1 & 1.7 \\
$H \rightarrow V V \rightarrow \ell \nu j j$ & 8.8 & 6.0 & 1.7 & 0.8 & 0.3 & 0.07 & 0.01 \\
$V H \rightarrow V V V \rightarrow \ell \nu j j j j$ & 7.3 & 4.5 & 1.6 & 0.9 & 0.3 & 0.05 & 0.01 \\
\hline Diboson & 1138 & 727 & 238 & 113 & 42 & 14 & 10 \\
$V+(g, u, d, s)$-jets & 24086 & 18078 & 4577 & 976 & 582 & 34 & 3 \\
$V+(b \bar{b} / c \bar{c})$ & 6625 & 3213 & 1349 & 1250 & 411 & 228 & 164 \\
top $(t \bar{t}+$ single top) & 3695 & 563 & 419 & 1123 & 365 & 460 & 570 \\
Multijet & 10364 & 6629 & 2162 & 933 & 367 & 130 & 82 \\
\hline Total expectation & 45908 & 29209 & 8746 & 4395 & 1768 & 867 & 830 \\
Total uncertainty & \pm 2582 & \pm 1619 & \pm 587 & \pm 528 & \pm 209 & \pm 118 & \pm 113 \\
Observed events & 45907 & 28924 & 8814 & 4278 & 1815 & 879 & 797 \\
\hline \hline
\end{tabular}

Table 6.4: Observed number of events in data and expected number of events from each signal and background source (where $V=W, Z$ ) for events with exactly three jets in muon and electron channels combined. The expected signal is quoted at $M_{H}=125 \mathrm{GeV}$. The total background uncertainty include the statistical and systematic uncertainties.

\begin{tabular}{lrrr}
\hline \hline & Pretag & 0 Tag & $1 \mathrm{~L}$ \\
\hline$V H \rightarrow \ell \nu b b$ & 1.4 & 0.2 & 0.2 \\
$H \rightarrow V V \rightarrow \ell \nu j j$ & 2.4 & 1.4 & 0.6 \\
$V H \rightarrow V V V \rightarrow \ell \nu j j j j$ & 3.6 & 2.0 & 0.8 \\
\hline Diboson & 199 & 112 & 46 \\
$V+(g, u, d, s)$-jets & 3055 & 2143 & 679 \\
$V+(b \bar{b} / c \bar{c})$ & 1280 & 542 & 286 \\
top $(t \bar{t}+$ single top $)$ & 2889 & 311 & 268 \\
Multijet & 2092 & 1110 & 450 \\
\hline Total expectation & 9516 & 4217 & 1729 \\
Total uncertainty & \pm 530 & \pm 231 & \pm 144 \\
Observed events & 9685 & 3915 & 1786 \\
\hline \hline
\end{tabular}

Table 6.5: Observed number of events in data and expected number of events from each signal and background source (where $V=W, Z$ ) for events with four or more jets in muon and electron channels combined . The expected signal is quoted at $M_{H}=125 \mathrm{GeV}$. The total background uncertainty includes all sources of systematic uncertainty added in quadrature. 

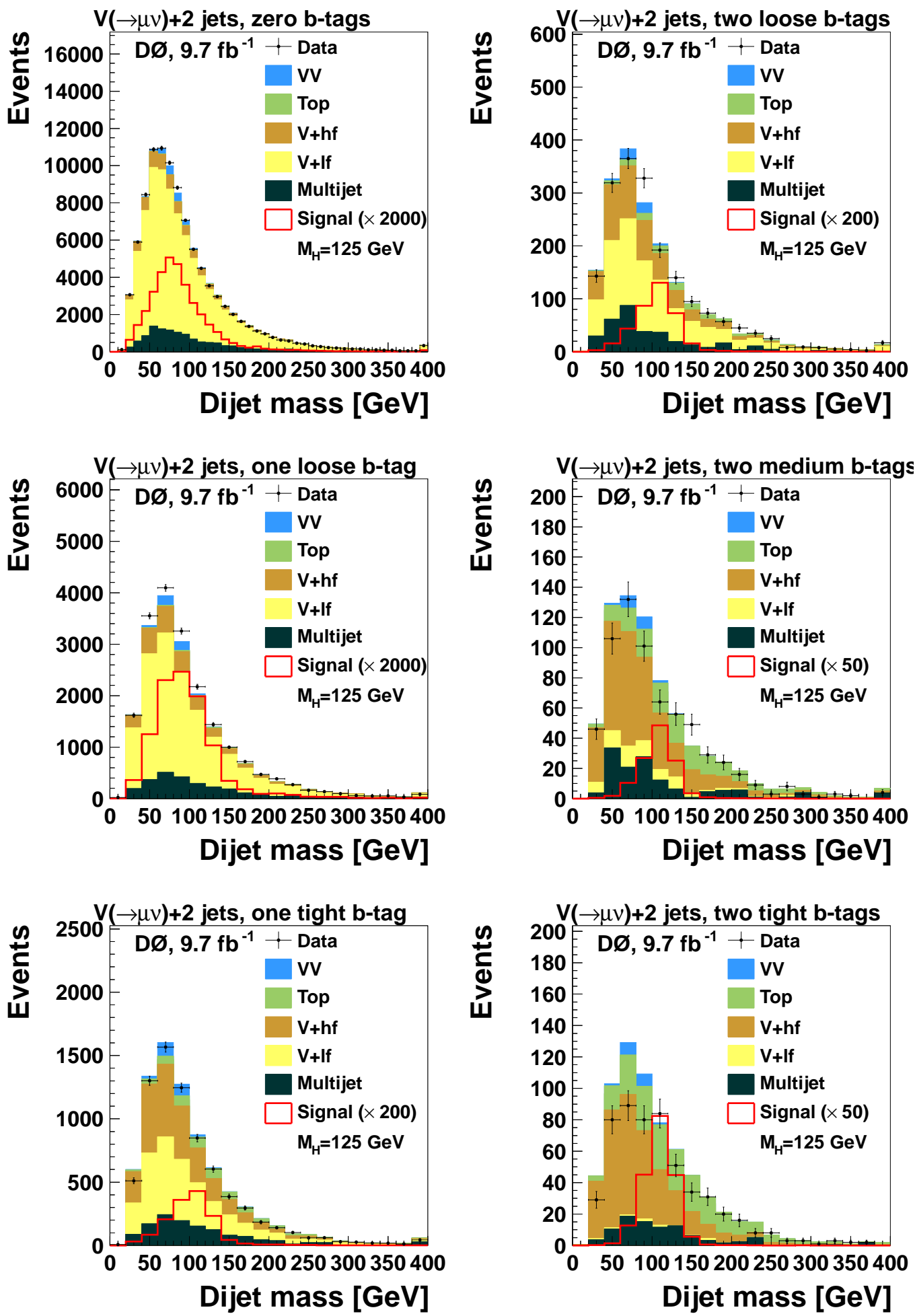

Figure 6.5: Distribution of invariant mass of the leading and second-leading jets for various $b$-tagging categories in events with two jets 


\subsubsection{Organizing the Search in $\ell \nu j j$ Final State}

The $W H \rightarrow \ell \nu b b$ process provides the dominant signal contribution in the one tight $b$-tag or two $b$-tag categories of the two or three jet channels. Since the branching fraction for the Higgs decay to $b$ quarks is only significant over the mass range $90-150 \mathrm{GeV}$, the search in the one tight and two-b-tag samples is restricted to this range of $M_{H}$.

In events with two or three jets and zero and one loose b-tag, the primary signal contribution is from the SM Higgs boson decay to a pair of $W$ bosons, $H \rightarrow W W \rightarrow \ell \nu j j$. Events in these jet multiplicity and $b$-tag categories are examined for $100 \leq M_{H} \leq 150 \mathrm{GeV}$. Since we do not perform the search in the one tight and two-b-tag samples for $M_{H}>150 \mathrm{GeV}$, events having exactly two or three jets in all $b$-tag categories (i.e. pretag events) are used in the search for $155 \leq M_{H} \leq 200 \mathrm{GeV}$.

\begin{tabular}{||c|c|c|c||}
\hline \hline N-Jets & 0 Tag & $1 \mathrm{~L}$ & $1 \mathrm{~T} / 2 \mathrm{~L} / 2 \mathrm{M} / 2 \mathrm{~T}$ \\
\hline 2 & $H \rightarrow W W \rightarrow \ell \nu j j$ & $H \rightarrow W W \rightarrow \ell \nu j j$ & $W H \rightarrow \ell \nu b \bar{b}$ \\
3 & $H \rightarrow W W \rightarrow \ell \nu j j$ & $H \rightarrow W W \rightarrow \ell \nu j j$ & $W H \rightarrow \ell \nu b \bar{b}$ \\
4 & $V H \rightarrow V W W \rightarrow \ell \nu j j j j$ & $V H \rightarrow V W W \rightarrow \ell \nu j j j j$ & --- \\
\hline \hline
\end{tabular}

Table 6.6: Assignment of $b$-tagging categories and jet multiplicities to sub-analyses to perform the SM Higgs search in the mass range $M_{H} \leq 150 \mathrm{GeV}$.

Only the zero and one-loose b-tag categories are considered when searching for the signal in events with four or more jets because $t \bar{t}$ production dominates the small amount of signal present in higher b-tag categories. The majority of signal events in the zero and one loose b-tag categories of the four-jet channel are from the $V H \rightarrow V W W \rightarrow \ell \nu j j j j$ process, and the SM Higgs search in this channel is performed over the mass range of $100-200 \mathrm{GeV}$.

The $\ell \nu j j$ analysis is organized into three sub-analyses namely $W H \rightarrow \ell \nu b b, H \rightarrow$ $W W \rightarrow \ell \nu j j$ and $V H \rightarrow V W W \rightarrow \ell \nu j j j j$, according to the main signal contribution. Table 6.6 details how the tagging categories and jet multiplicities are assigned to each subanalysis in the search for the SM Higgs boson in the low mass range $90 \leq M_{H} \leq 150 \mathrm{GeV}$.

\subsection{Event Selection in $Z H \rightarrow \ell \ell b b$ Analysis}

The $Z H \rightarrow \ell \ell b b$ preselection requires a reconstructed $Z$ candidate decaying as $Z \rightarrow \mu \mu$ or $Z \rightarrow e e$ plus at least two additional jets [11]. The search in this analysis is performed in four independent channels defined by the subdetectors used for lepton identification: the dimuon channel $(\mu \mu)$, the muon + isolated track channel $\left(\mu \mu_{\text {trk }}\right)$, the dielectron channel $(e e)$, and the electron + ICR electron channel $\left(e e_{\mathrm{ICR}}\right)$.

\subsubsection{Event Triggering}

In the $\mu \mu$ and $\mu \mu_{\text {trk }}$ channels, we accept events that satisfy any trigger requirement. As in the $\ell \nu j j$ analysis, the efficiency of this inclusive trigger is obtained by applying a trigger 
correction to the efficiency of the well-modeled single-muon trigger suite.

In the ee channel we also accept events that satisfy any trigger requirement, with a measured efficiency consistent with 100\%. Therefore, no correction for the trigger efficiency is applied to the event weights in the ee channel. The set of triggers used in the $e e_{\mathrm{ICR}}$ channel has an efficiency of $90-100 \%$ depending on the region of the detector, and the efficiency in this channel is parametrized in electron $\eta$, electron $\phi$, and jet transverse momentum [11].

\subsubsection{Offline Event Selection}

\section{Primary vertex selection}

The event selection in all four channels of the $Z H \rightarrow \ell \ell b b$ analysis requires a primary $p \bar{p}$ interaction vertex that has $P V_{z} \leq 60 \mathrm{~cm}$.

\section{Jet selection}

All events considered in the $Z H \rightarrow \ell \ell b b$ analysis must contain at least two jets originating from the primary $p \bar{p}$ vertex, that satisfy the jet identification criteria (Sec. 4.2.3) and following additional requirements:

- jet $p_{T} \geqslant 20 \mathrm{GeV}$ and $\left|\eta_{\text {det }}\right| \leq 2.5$;

- jets must be taggable.

\section{Lepton selection}

\section{Dielectron selection (ee)}

In the dielectron channel the lepton selection requires events to have:

- at least two electrons ${ }^{1}$ reconstructed either in the $\mathrm{CC}$ with $\left|\eta_{\text {det }}\right|<1.1$ or in the EC with $1.5<\left|\eta_{\text {det }}\right|<2.5$, that satisfy the CC or EC identification criteria listed in Table 4.2.1;

- at least one electron identified in the CC;

- at least two electron candidates with $p_{T}>15 \mathrm{GeV}$ and opposite charges.

Electron-plus-ICR selection (e $\left.\boldsymbol{e}_{\mathbf{I C R}}\right)$

The lepton selection requires events in the $e e_{\mathrm{ICR}}$ channel to have exactly one electron passing the identification criteria in either the $\mathrm{CC}$ or $\mathrm{EC}$ with $p_{T}>15 \mathrm{GeV}$ and one electron reconstructed in the ICR $\left(1.1<\left|\eta_{\text {det }}\right|<1.5\right)$ which must be matched to a calorimeter energy deposit with $E_{T}>15 \mathrm{GeV}$. The two electron candidates must have opposite charges.

Dimuon selection $(\boldsymbol{\mu} \boldsymbol{\mu})$

The dimuon channel includes events with:

\footnotetext{
${ }^{1}$ Throughout the text, we refer to both electrons and positrons as "electrons". Likewise, we refer to both muons and anti-muons as "muons".
} 
- at least two muon candidates passing medium quality track and loose muon isolation criteria defined in Sec. 4.2.2 and having opposite charges;

- at least two muons with $p_{T}>10 \mathrm{GeV}$ and $\left|\eta_{\text {det }}\right| \leq 2$;

- at least one muon with $p_{T}>15 \mathrm{GeV}$ and $\left|\eta_{\text {det }}\right| \leq 1.5$;

- at least one muon satisfying the tight muon isolation criteria (Sec. 4.2.2).

Muon-plus-track selection $\left(\boldsymbol{\mu} \boldsymbol{\mu}_{\mathrm{trk}}\right)$

The $\mu \mu_{\text {trk }}$ channel is designed to recover dimuon events in which one muon is not identified in the muon system, primarily because of gaps in the muon system coverage. In this channel events are required to have:

- exactly one muon with $\left|\eta_{\text {det }}\right|<1.5$ and $p_{T}>15 \mathrm{GeV}$, which must satisfy the criteria for the medium-quality track and tight muon isolation, and an isolated track with $\left|\eta_{\text {det }}\right|<2$ and $p_{T}>20 \mathrm{GeV}$, separated from the muon by $\Delta \mathcal{R}>0.1$;

- the track-only muon $\left(\mu_{\text {trk }}\right)$ must also satisfy the same tracker and calorimeter isolation requirements as the first muon;

- the $\mu$ and the $\mu_{\text {trk }}$ candidates must have opposite charges.

To ensure that the $\mu \mu$ and $\mu \mu_{\text {trk }}$ selections do not overlap, events that contain any additional muons with $\left|\eta_{\text {det }}\right|<2$ and $p_{T}>10 \mathrm{GeV}$ are rejected by the $\mu \mu_{\text {trk }}$ selection.

\section{Reconstructed $Z$ selection}

A good $Z$ candidate is required in each event selected for the $Z H \rightarrow \ell \ell b b$ analysis. The $Z$ candidate reconstructed from a pair of selected electrons or muons $(Z \rightarrow \ell \ell)$ must have an invariant mass satisfying $70<M_{\ell \ell}<110 \mathrm{GeV}$.

\subsubsection{Multijet Background and Normalization at the Preselection}

The multijet background in $Z H \rightarrow \ell \ell b b$ is estimated from control samples in the data. The conventional approach to derive the multijet template is to reverse preselection criteria that are used to reject multijet background. In the dimuon $(\mu \mu)$ channel, a multijet event must contain a $Z$ candidate that passes all event selection requirements except isolation and that fails the opposite sign requirement. In the muon-plus-track $\left(\mu \mu_{\text {trk }}\right)$ channel, multijet events are defined as those that pass all selection criteria, except the $\mu$ and $\mu_{\text {trk }}$ have samesign charges. For the ee channel, the electron isolation and shower shape requirements are reversed. The multijet sample for the $e e_{\mathrm{ICR}}$ channel consists of events that satisfy the signal selection, but have inverted quality requirements on the ICR electron. 
For each lepton channel, the normalization of the multijet background and all simulated samples are adjusted using a simultaneous template fit of the dilepton mass $\left(M_{\ell \ell}\right)$ distributions in each channel and jet multiplicity bins. The fit minimizes the $\chi^{2}$ :

$$
\chi^{2}=\sum_{i, j, m} \frac{\left(D_{m}^{i j}-\alpha^{i j} \cdot Q_{m}^{i j},-k_{\epsilon}^{i} \cdot\left(k_{Z}^{j} \cdot Z_{m}^{i j}+O_{m}^{i j}\right)\right)^{2}}{D_{m}^{i j}}
$$

where $m$ runs over the bins of $M_{\ell \ell}, j$ runs over the jet multiplicity, and $i$ indicates the channel. The number of data events are $D_{m}^{i j}$, and the fit adjusts the normalization of $Q_{m}^{i j}$, the multijet sample, $Z_{m}^{i j}$, the simulated $Z$ boson (including $Z+b \bar{b}$ and $Z+c \bar{c}$ ) sample, and $O_{m}^{i j}$, all other simulated samples. The fit parameters are the multijet scale factors $\alpha^{i j}$ that apply to $Q_{m}^{i j}$, the combined luminosity and efficiency scale factors $k_{\epsilon}^{i}$ for channel $i$ that are applied to $Z_{m}^{i j}$ and $O_{m}^{i j}$, and the $Z$ boson cross section scale factors $k_{Z}^{j}$ that apply to $Z_{m}^{i j}$.

The dimuon and dielectron mass spectra for the electron and muon channels after the normalization procedure are shown in Fig. 6.6. Figures 6.7 and 6.8 show distributions of the transverse momenta of the two jets with the highest $p_{T}$ and the invariant mass of the dijet system constructed from those two jets. In all plots, data points are shown with error bars that reflect statistical uncertainty only, and discrepancies in data-MC agreement are within the systematic uncertainties described in Sec. 8.2.

\subsubsection{Classifying $b$-tag Samples}

In order to distinguish events containing a $H \rightarrow b \bar{b}$ decay from background processes involving light quarks $(u d s), c$ quark and gluons, the jets of preselected (pretag) events are identified as "loose" or "tight" b-tagged if they passed corresponding pre-defined loose or tight selection requirement on the $b$-jet identification discriminant output, which are listed in Table 4.3 (Sec. 4.2.5). Events with at least one tight and one loose $b$ tag are classified as double-tagged (DT). If an event fails the DT requirement, but contains a single tight $b$ tag, it is classified as single-tagged (ST). Table 6.7 gives the number of events observed in the pretag, ST and DT samples, and the expected number of events for the different background components and the signal (assuming $M_{H}=125 \mathrm{GeV}$ ), following all $\mathrm{MC}$ corrections and the preselection normalization.

The $H \rightarrow b \bar{b}$ candidate is composed of the two highest- $p_{T}$ tagged jets in DT events, and the tagged jet plus the highest- $p_{T}$ non-tagged jet in ST events. Figure 6.9 shows the distribution of the dijet invariant mass $\left(M_{b b}\right)$ in ST and DT events. The dijet mass resolution of the signal is better in the DT sample (Fig. 6.9(b)) than in the ST sample (Fig. 6.9(a)) due to lower levels of contamination in the DT sample from jets that are not associated with the $H \rightarrow \bar{b}$ decay. 

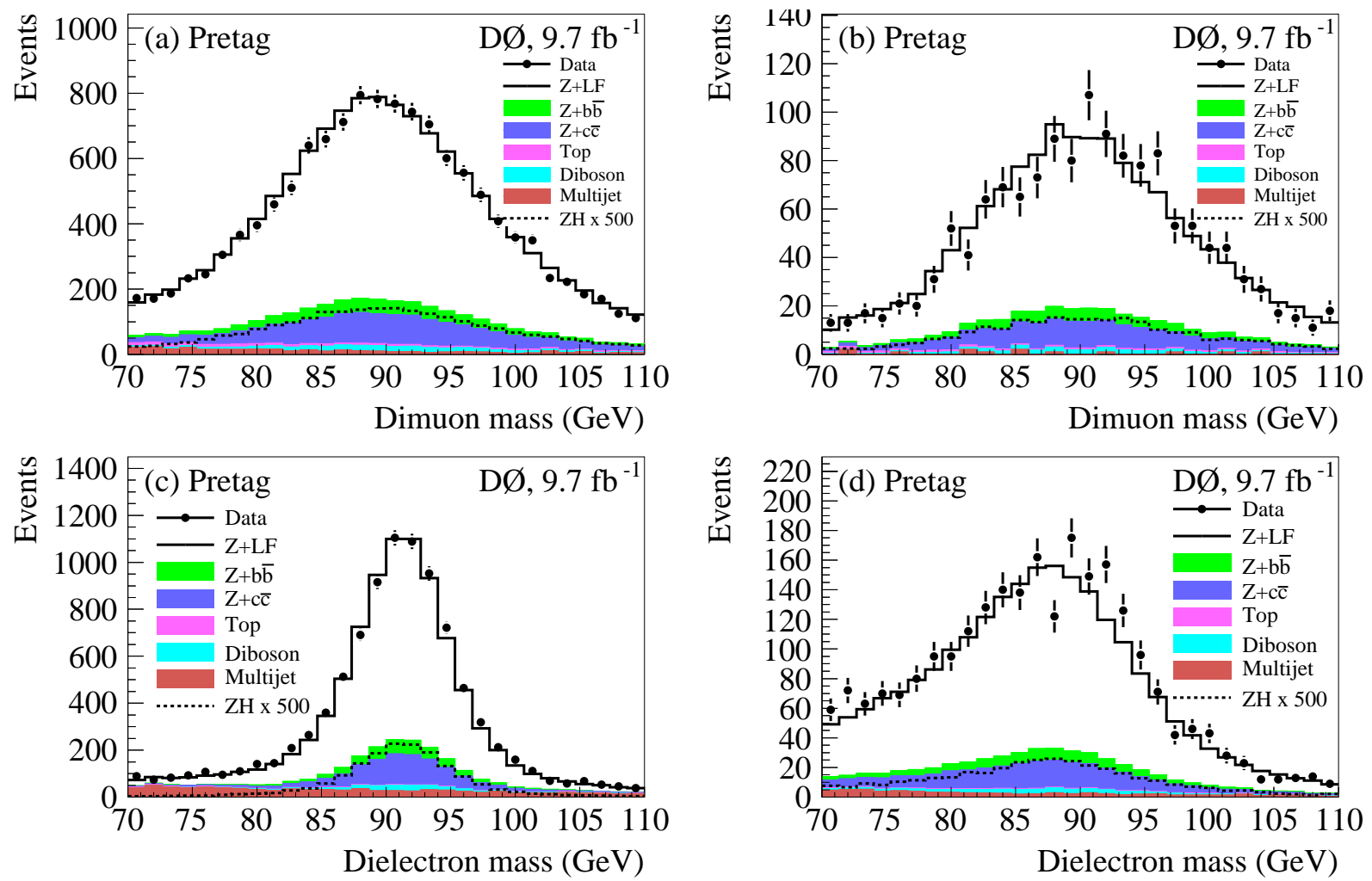

Figure 6.6: The dilepton mass spectra, along with the background expectation, for the (a) $\mu \mu$, (b) $\mu \mu_{\mathrm{trk}}$, (c) $e e$ and (d) $e e_{\mathrm{ICR}}$ channels in the pretag sample. Signal distributions for $M_{H}=125 \mathrm{GeV}$ are scaled by a factor of 500 .
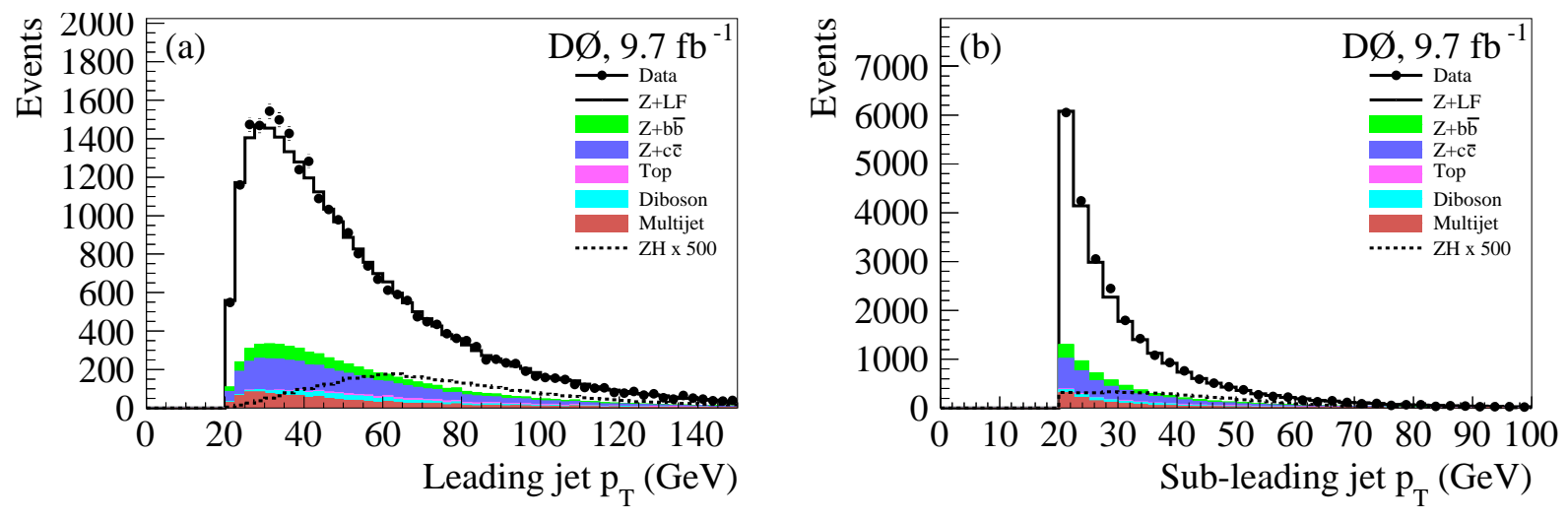

Figure 6.7: The $p_{T}$ spectra of the (a) leading and (b) sub-leading jets, along with the background expectations, summed over all lepton channels in the pretag sample. Signal distributions, for $M_{H}=125 \mathrm{GeV}$, are scaled by a factor of 500 . 


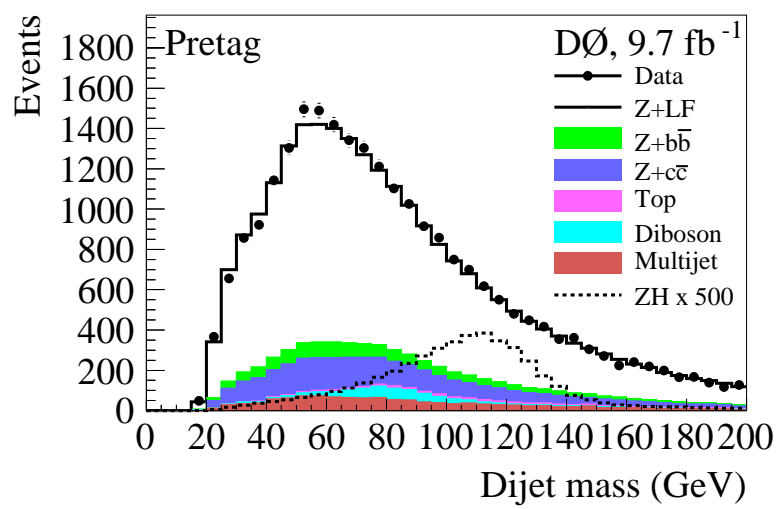

Figure 6.8: Distribution of the dijet invariant mass, along with the background expectation, summed over all lepton channels in the pretag sample. The signal distribution, for $M_{H}=$ $125 \mathrm{GeV}$, is scaled by a factor of 500 .
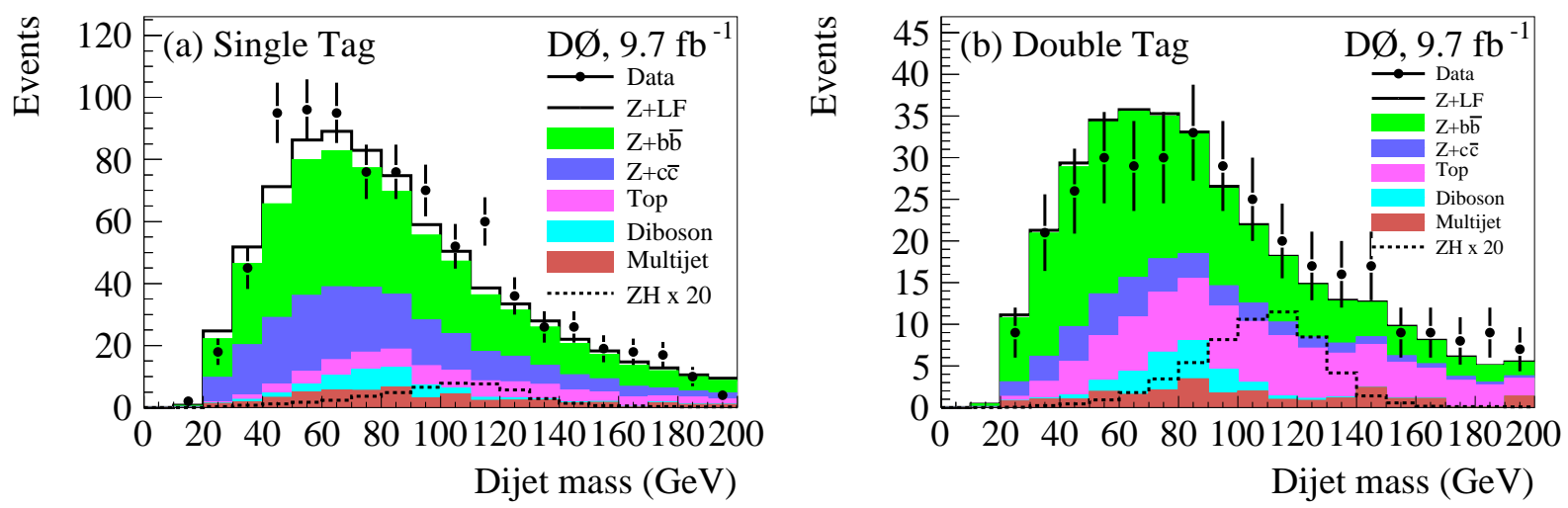

Figure 6.9: Distribution of dijet invariant mass in (a) ST and (b) DT events. Signal distributions for $M_{H}=125 \mathrm{GeV}$ are scaled by a factor of 20 .

\begin{tabular}{lcccccccc}
\hline \hline & Data & Total Background & MJ & $Z+\mathrm{LF}$ & $Z+\mathrm{HF}$ & Diboson & $t \bar{t}$ & $Z H$ \\
\hline Pretag & 25849 & 25658 & 1284 & 19253 & 4305 & 530 & 285 & 9.2 \\
ST & 886 & $824 \pm 102$ & 54 & 60 & 600 & 33 & 77 & 2.5 \\
DT & 373 & $366 \pm 39$ & 25.7 & 3.5 & 219 & 19 & 99 & 2.9 \\
\hline \hline
\end{tabular}

Table 6.7: Expected and observed event yields for all lepton channels combined after requiring two leptons, at least two jets and $70<M_{\ell \ell}<110 \mathrm{GeV}$ (pretag), and after requiring exactly one (ST) or at least two (DT) $b$ tags. The $Z H$ yields are given for $M_{H}=125 \mathrm{GeV}$. The uncertainties quoted include the statistical and systematic uncertainties. 


\section{7 \\ Multivariate Signal Discriminants}

After imposing selection requirements to enhance signal purity in data, the search for the SM Higgs boson is still very challenging because of the presence of prodigious background at the Tevatron. In order to increase the signal sensitivity, multivariate analysis (MVA) techniques are used to fully exploit the kinematic properties of the SM Higgs boson production and decay processes in each search channel and incorporate signal-to-background discriminating information from individual event kinematic variables into a single discriminating variable, the MVA signal discriminant. A crucial process in our analyses is optimizing the MVA performance for each search channel to maximize the power of the MVA signal discriminant (referred to as MVA discriminant from here after) in differentiating signal and background events mixed in a data sample.

Two MVA methods, the decision tree and the matrix element, are employed in the search for the SM Higgs signal in the lepton plus jets final states. While the decision tree method is based on a machine learning approach, the matrix element (ME) method explicitly relies on the physics calculations of scattering amplitudes and phase space of signal and background processes. For the $\ell \nu j j$ analysis, we use a random forest (RF) of decision trees [48] implemented in the SPR package $[100,101]$ for events in the zero and one loose $b$-tag channel, and we use a boosted decision tree (BDT) implemented with the TMVA package [102] in the one tight $b$-tag and all three two $b$-tag channels. For the $Z H \rightarrow \ell \ell b b$ analysis, we use both the ME method and the random forest of decision trees implemented in the TMVA software package [102] for discriminating signal from background. To maximize the use of kinematic information in the RF for $Z H \rightarrow \ell \ell b b$ analysis, we employ a kinematic fit to improve the resolution of the invariant mass of the Higgs boson candidate reconstructed from the jets included in the final state. The RF discriminants provided by the decision tree method are used for setting limits on the signal production, and the ME discriminant derived by ME method is used to verified the result obtained from the decision tree method.

The next sections focus on describing MVA optimizations that the author of this thesis has worked on. The multivariate analysis in $H \rightarrow W W \rightarrow \mu \nu j j$ channel, which is composed of events in the zero and one loose $b$-tag categories of muon channel, is described first. Then 
the ME approach, the kinematic fit process and the RF discriminants in the $Z H \rightarrow \ell \ell b b$ are presented.

\subsection{Mutivariate Analysis in $H \rightarrow W W \rightarrow \mu \nu j j$ Channel}

We use the random forest of decision trees as a MVA classifier to discriminate the SM Higgs signal and background events in the $H \rightarrow W W \rightarrow \mu \nu j j$ channel. The procedure of optimization and estimation of the performance of the RF classifier proceeds in three stages: training, validation and testing. Monte Carlo signal and background events along with multijet events are used in each stage. Depending on the size of the sample, up to $1 / 3$ of these events are used to train the RF classifier. To avoid potential bias, a smaller, independent sample of events is reserved for validation of the performance of the RF and to compare the performance of classifiers trained under different scenarios before considering the $\mathrm{RF}$ response distributions used in the analysis. Finally, the response of the optimized RF classifier is evaluated on a testing sample consisting of the remaining signal and background events and these results are used to construct the MVA discriminant.

\subsubsection{Random Forest of Decision Trees}

The random forest used in our analysis is composed of a collection of decision trees where each tree is trained to separate signal from background. Given a list of input variables $\left\{x_{i}\right\}$, a decision tree starts with all events in its assigned training data set and finds a binary split for each variable that optimizes the signal-to-background separation, according to some chosen criterion, and then selects the variable and its corresponding split that has the best separation power to split the input data into two new nodes, signal enriched and background enriched. The decision tree continues to examine each of the new nodes and splits them into finer nodes. The splitting procedure is repeated until a stopping criterion is satisfied. A terminal node created when the splitting stops is called a "leaf". Each leaf is associated with a signal purity defined as the weighted signal fraction of the total data in the leaf. Once a tree is built, a new event can be run through the tree and its kinematic properties are compared to the splitting tests until it reaches a leaf, and the output of the tree for this event is the leaf purity.

Each binary split in our analysis is optimized using the negative Gini index $[100,101]$ as a figure of merit:

$$
G=-p(1-p)=-\frac{s b}{(s+b)^{2}},
$$

where s (b) is the sum of signal (background) weights and $p=s /(s+b)$ is the signal purity at the corresponding node. The negative Gini index is minimal for an equal mix of signal and background in the node (no separation) and is maximal when there are either only signal or only background events in the node (perfect separation). A split is optimized to 
give the largest overal figure of merit:

$$
G_{\text {split }}=\frac{W^{(1)} G_{1}+W^{(2)} G_{2}}{W}
$$

where $\mathrm{W}$ is the total weight of events in the parent node, which is split in two daughter nodes with weights $W^{(1)}$ and $W^{(2)}\left(W=W^{(1)}+W^{(2)}\right)$. A node is not split further and becomes a leaf if the improvement in the initial figure of merit $G$ is negligible or the best split would create a daughter node with fewer than a minimum number of events. The minimum number of events per a leaf (the leaf size) is chosen to guarantee statistical significance of the associated purity.

The RF is constructed from multiple decision trees where each tree is trained on a randomly selected collection of signal and background MC events as well as multijet events from the full training data set. Each subset of training data is sampled using Bootstrap AGGregatING (commonly referred to as "bagging") technique [101] in such a way that it has the same size as the full training data set, but may contain duplicate events. In addition, a random subset of input variables are examined to split each node of the tree. The result is that each decision tree in the $\mathrm{RF}$ is trained with slightly different input variables and training events. Output from the random forest is then constructed by averaging the output from each decision tree. Given a new event, each decision tree in the forest evaluates the kinematic information of the event and supplies an output, and the output of the random forest for the event is the mean of all the decision tree outputs.

\subsubsection{Optimizing Random Forest Classifiers}

To obtain maximal separation of signal and background events in $H \rightarrow W W \rightarrow \mu \nu j j$ channel, we optimize the RF classifier separately for each jet multiplicity, $b$-tagging category and for each hypothesized SM Higgs boson mass. For the search in the mass range $100 \leq M_{H} \leq$ $150 \mathrm{GeV}$, the RF classifier is optimized separately for each zero $b$-tag or one loose $b$-tag sample with two jets or three jets and for each mass point in steps of $5 \mathrm{GeV}$. For the search in the mass range $155 \leq M_{H} \leq 200 \mathrm{GeV}$, all pretag events are analyzed and we optimize a RF classifier for each of two-jets and three-jets samples and for each mass point in steps of $5 \mathrm{GeV}$. In order to focus on optimizing the splitting at nodes dominated by signal, the signal events in the training sample are scaled up to make the total weighted signal integral equal to the total weighted background integral. A number of parameters characterizing the structure of the RF, such as input variables, the number of decision trees and the leaf size, are optimized to improve the performance of the RF. Among them, the choice of input variables is the most crucial for the discriminating power of the RF classifier and so is the main focus of the RF optimization process. 


\section{Input variable selection}

The performance of a RF classifier depends on the signal-to-background discriminating power of input variables used for training the decision trees. In order to find those variables, we start with defining more than two hundred physics-driven variables to describe the kinematics of individual objects in the $\mu \nu j j$ final state, angular correlations between the objects, and global event kinematics such as reconstructed masses and event shapes. We then scan through the full list to search for variables which have strong signal-to-background discriminating power and are well modeled. The Kolmogorov-Smirnov (KS) distance, which measures the difference in shape between the normalized signal and background distributions corresponding to each of defined variables, is evaluated and we require input variables for the RF to have the KS distance greater than a threshold. To ensure a good agreement in describing data and total of $\mathrm{MC}$ and multijet events using a selected input variable, we require the $\chi^{2}$ probability between data and total background distributions of that variable to be greater than $5 \%$.

The importance of variables used in the RF classifier and the correlations between them are evaluated on the validation sample by investigating how often the variables are used to split decision tree nodes and how the discriminating power of the RF classifier decreases when each variable is removed from the tree. To estimate the change in discriminating power of the RF, we use the average quadratic loss $(\Lambda)$ in the validation sample as a figure of merit:

$$
\Lambda=\frac{\sum_{i=1}^{N_{\mathrm{v}}} w_{i}\left(x_{i}-y_{i}\right)}{\sum_{i=1}^{N_{\mathrm{v}}} w_{i}},
$$

where $N_{\mathrm{v}}$ is the total number of events in the validation sample, $x_{i}$ is desired output of the $\mathrm{RF}$ for the $i$ th event ( $x_{i}$ is zero for background events, one for signal events), $w_{i}$ and $y_{i}$ are the weight and the RF output for that event. The quadratic loss measures how far the RF output is from the desired output, and smaller loss means better signal/background classification.

Because the signal shape is driven by the signal mass hypothesis, we optimize the MVA variable list at two different mass points: at $M_{H}=125 \mathrm{GeV}$ for masses below $150 \mathrm{GeV}$ and at $M_{H}=165 \mathrm{GeV}$ for masses above $150 \mathrm{GeV}$. Since the resolution of the reconstructed Higgs boson mass is about $20 \mathrm{GeV}$ for $H \rightarrow W W \rightarrow \mu \nu j j$ channel, optimizing the input variable list at only these mass points is sufficient. These regions also correspond to the distinct cases of off-shell versus on-shell final states of the second $W$ boson. Tables 7.1 and 7.2 list the input variables of the RF optimized for each jet multiplicity and $b$-tagging category for each search in the low mass $\left(100 \leq M_{H} \leq 150 \mathrm{GeV}\right)$ and high mass $\left(155 \leq M_{H} \leq 200 \mathrm{GeV}\right)$ regions. Distributions of selected input variables for each jet multiplicity and $b$-tag sub-channel are 
shown in Figs. 7.1-7.3. A description of all selected variables is given below:

- $\mu_{p_{T}}$ - transverse momentum of muon

- $\nu_{p_{T}}$ - missing transverse energy $\mathbb{E}_{T}$

- $p_{T}^{j_{1}}$ - transverse momentum of the leading jet in $p_{T}$

- $p_{T}^{j_{2}}$ - transverse momentum of the second leading jet in $p_{T}$

- $p_{T}^{j_{3}}$ - transverse momentum of the third leading jet in $p_{T}$

- $p_{T}^{W_{l e p}}$ - transverse momentum of the $W(\rightarrow \mu \nu)$ system

- $p_{T}^{j_{1} j_{2}}$ - transverse momentum of the leading two jets (dijet system)

- $p_{T}^{j_{1} j_{2} j_{3}}$ - transverse momentum of the system of three leading jets

- $p_{T_{\text {recoil }}}^{j_{1} j_{2}}$ - transverse momentum of the dijet system with respect to the thrust vector $\left.\Delta \vec{P}\left(j_{1}, j_{2}\right)=\vec{P}_{j_{1}}-\vec{P}_{j_{2}}\right)$

- $\left(p_{T}^{W_{l e p}}\right) /\left(\mu_{p_{T}}+\nu_{p_{T}}\right)$ - ratio of transverse momentum the $W(\rightarrow \mu \nu)$ system and the scalar sum of transverse momentum of $\mu$ and $\nu$

- $\left(p_{T}^{j_{1} j_{2}}\right) /\left(p_{T}^{j_{1}}+p_{T}^{j_{2}}\right)$ - ratio of transverse momentum of the dijet system and the scalar sum of transverse momentum of the two leading jets

- $\left(p_{T}^{j_{2} j_{3}}\right) /\left(p_{T}^{j_{2}}+p_{T}^{j_{3}}\right)$ - ratio of transverse momentum of the dijet system and the scalar sum of transverse momentum of the second and the third leading jets

- $p_{T_{\text {recoil }}}^{W_{\text {lep }}}$ - transverse momentum of the $W(\rightarrow \mu \nu)$ system with respect to the thrust vector $\Delta \vec{P}(\mu, \nu)=\vec{P}_{\mu}-\vec{P}_{\nu}$

- $\Delta p_{T}(\mu, \nu)$ - difference in $p_{T}$ between $\mu$ and $\nu$

- $\Delta p_{T}\left(W_{l e p}, \mu\right)$ - difference in $p_{T}$ between the $W(\rightarrow \mu \nu)$ system and $\mu$

- $\Delta \eta\left(j_{1}, \mu\right)-\Delta \eta$ between $\mu$ and the leading jet

- $\Delta \phi\left(W_{l e p}, j_{2}\right)-\Delta \phi$ between the second leading and the $W(\rightarrow \mu \nu)$ system

- $\Delta \phi\left(W_{l e p}, j_{12}^{b i s}\right)-\Delta \phi$ between the bisector of the dijet system and $W(\rightarrow \mu \nu)$

- $\Delta \phi\left(j_{1}, j_{3}\right)-\Delta \phi$ between the first leading and the third leading jets

- $\Delta \phi\left(j_{2} j_{3}, j_{3}\right)-\Delta \phi$ between the third leading jets and the system of the second and the third jets

- $\min \left[\Delta \phi\left(W_{l e p}, \mu\right.\right.$ or $\left.\left.\nu\right)\right]$ - minimum $\Delta \phi$ between the $W(\rightarrow \mu \nu)$ system and $\mu$ or $\nu$ 
- $\Delta R\left(j_{1}, j_{2}\right)-\Delta R$ between the leading and second leading $p_{T}$ jets

- $\Delta R\left(j_{1}, \mu\right)-\Delta R$ between $\mu$ and the leading jet

- $\Delta R\left(W_{\text {lep }}, \nu\right)-\Delta R$ between the $\nu$ and the $W(\rightarrow \mu \nu)$ system

- $\Delta R\left(W_{l e p}, j_{2}\right)-\Delta R$ between the second leading jet and the $W(\rightarrow \mu \nu)$ system

- $\Delta R\left(W_{\text {lep }}, j_{1}\right)-\Delta R$ between the leading jet and the $W(\rightarrow \mu \nu)$ system

- $\min \left[\Delta R\left(W_{\text {lep }}, \nu\right.\right.$ or $\left.\mu\right)$ - minimum $\Delta R$ between $W(\rightarrow \mu \nu)$ system and $\mu$ or $\nu$

- $\cos \theta\left(W_{\text {lep }}, \mathcal{H}^{C M}\right)-\cos \theta$ of the $W(\rightarrow \mu \nu)$ system in the reconstructed $H(\rightarrow W W \rightarrow$ $\mu \nu j j)$ CM frame

- $\cos \theta\left(j_{1} j_{2}, \mathcal{H}^{C M}\right)-\cos \theta$ of the dijet system in the reconstructed $H(\rightarrow W W \rightarrow \mu \nu j j)$ CM frame

- $3 D$ angle $\left(\vec{P}_{\mu}, \vec{P}_{\nu}\right)$

- $3 D$ angle $\left(\vec{P}_{j_{1}}, \vec{P}_{j_{2}}\right)_{\mathcal{H}^{C M}}$ - 3D angle between the two leading jets in the reconstructed $W(\rightarrow \mu \nu) \mathrm{CM}$ frame

- $M_{W_{l e p}}$ - invariant mas of the reconstructed $W(\rightarrow \mu \nu)$ system

- $M_{W_{l e p}}^{T}$ - transverse mas of the $W(\rightarrow \mu \nu)$ system

- $M_{j_{1} j_{2}}$ - reconstructed invariant mass of the two leading $p_{T}$ jets (dijet mass)

- $M_{j_{1} j_{2}}^{T}$ - transverse mass of the two leading $p_{T}$ jets (dijet mass)

- $M_{\mu j_{1} j_{2}}$ - reconstructed invariant mass of the two leading $p_{T}$ jets and $\mu$

- $M_{\mu \nu j j}$ - invariant mass of the system consisting of the $\mu, \nu$ and two leading jets

- $M_{j_{1} j_{2} j_{3}}$ - invariant mass of the system of three leading jets

- $M_{\mu E_{T} j j}$ - invariant mass of a system consisting of the $E_{T}, \mu$ and two leading jets

- $M_{\mu \nu j j}^{a s y m}$ - invariant mass asymmetry between the $W(\rightarrow \ell \nu)$ system and the dijet system, defined as $\frac{M_{W_{l e p}}-M_{j_{12}}}{M_{W_{l e p}}+M_{j_{12}}}$

- $H_{j_{2} W_{l e p}}^{T}$ - the scalar sum of transverse momentum of the $W(\rightarrow \ell \nu)$ system and the second leading jet

- $H_{\mu j_{1} j_{2}}^{T}$ - the scalar sum of transverse momentum of the dijet system and $\mu$

- $H_{V I S}^{T}$ - the scalar sum of transverse momentum of $\mu$ and all jets 
- $H_{j_{2} W_{l e p}}^{T}$ - the scalar sum of transverse momentum of the $W(\rightarrow \ell \nu)$ system and the second leading jet

- $\left(H_{\mu \nu}^{T}\right) /\left(H_{j_{1} j_{2}}^{T}\right)$ - defined as $\frac{\mu_{p_{T}}+\nu_{p_{T}}}{p_{T}^{j_{1}}+p_{T}^{j_{2}}}$

- $H_{\mu \nu j j}^{T^{a s y m}}$ - asymmetry of scalar transverse momentum between the $W(\rightarrow \ell \nu)$ system and the dijet system, defined as $\frac{H_{W_{l e p}}^{T}-H_{j_{12}}^{T}}{H_{W_{l e p}}^{T}+H_{j_{12}}^{T}}$

- $W_{l e p}^{\Sigma_{\nu}}$ - defined as $\frac{\Delta R(\mu, \nu) \times \nu_{p_{T}}}{\mu_{p_{T}}+\nu_{p_{T}}}$

- $W_{l e p}^{\Sigma_{\mu}}$ - defined as $\frac{\Delta R(\mu, \nu) \times \mu_{p_{T}}}{\mu_{p_{T}}+\nu_{p_{T}}}$

- $\min \left[W_{l e p}^{\Sigma_{\mu}}, W_{l e p}^{\Sigma_{\nu}}\right]-\operatorname{minimum}$ of $W_{l e p}^{\Sigma_{\mu}}$ and $W_{l e p}^{\Sigma_{\nu}}$

- $j_{12}^{\Sigma_{j_{2}}}$ - defined as $\frac{\Delta R\left(j_{1}, j_{2}\right) \times p_{T}^{j_{2}}}{p_{T}^{j_{1}}+p_{T}^{j_{2}}}$

- $j_{12}^{\Sigma_{j_{1}}}$ - defined as $\frac{\Delta R\left(j_{1}, j_{1}\right) \times p_{T}^{j_{1}}}{p_{T}^{j_{1}}+p_{T}^{j_{2}}}$

- $\min \left[j_{12}^{\Sigma_{j_{1}}}, j_{12}^{\Sigma_{j_{2}}}\right]-\operatorname{minimum}$ of $j_{12}^{\Sigma_{j_{1}}}$ and $j_{12}^{\Sigma_{j_{2}}}$

- $j_{12}^{\Sigma}$ - defined as $\frac{\Delta R\left(j_{1}, j_{2}\right) \times p_{T}^{j_{1}}+\Delta R\left(j_{1}, j_{2}\right) \times p_{T}^{j_{2}}}{p_{T}^{j_{1}}+p_{T}^{j_{2}}}$

- $\mathcal{T} \mathcal{O} \mathcal{P O} \mathcal{O}_{\mu \nu \text { jr }}^{\text {centity }}$ - centrality, a topological variable describing the event shape, defined as $\left(\sum_{i} p_{T}^{i}\right) /\left(\sum_{i}\left|\vec{p}^{i}\right|\right)$ where $i$ runs over all objects in the final state.

- $\mathcal{T} \mathcal{O P O} \mathcal{O}_{\mu \nu j j}^{\text {sphericity }}$ - sphericity, a topological variable describing the event shape, measures the sum of squared transverse momentum of objects in the final state with respect to the event axis.

- $\mathcal{T} \mathcal{O P O} \mathcal{O}_{\mu j_{1} j_{2}}^{\text {sphericity }}$ - sphericity calculated for the system of $\mu$ and two leading jets

- $\mathcal{F} \mathcal{W} \mathcal{M}_{j_{1} j_{2} j_{3}}$ - The Fox-Wolfram moment, an event shape variable describing the correlations of four-momentum jets, defined as:

$$
\mathcal{F W M}_{j_{1} j_{2} j_{3}}=\frac{\sum_{i=1,2,3} p_{T}^{j_{i}} \times \Delta R\left(\vec{P}_{j_{i}}, \vec{P}_{j_{i} j_{2} j_{3}}\right)}{\sum_{i=1,2,3} p_{T}^{j_{i}}}
$$

- $\mathcal{F} \mathcal{W} \mathcal{M}_{j_{1} j_{2} j_{3}}^{l e p}$ - The Fox-Wolfram moment, defined with respect to the muon's axis:

$$
\mathcal{F} \mathcal{W M}_{j_{1} j_{2} j_{3}}^{\text {lep }}=\frac{\sum_{i=1,2,3} p_{T}^{j_{i}} \times \Delta R\left(\vec{P}_{j_{i}}, \vec{P}_{l e p}\right)}{\sum_{i=1,2,3} p_{T}^{j_{i}}}
$$




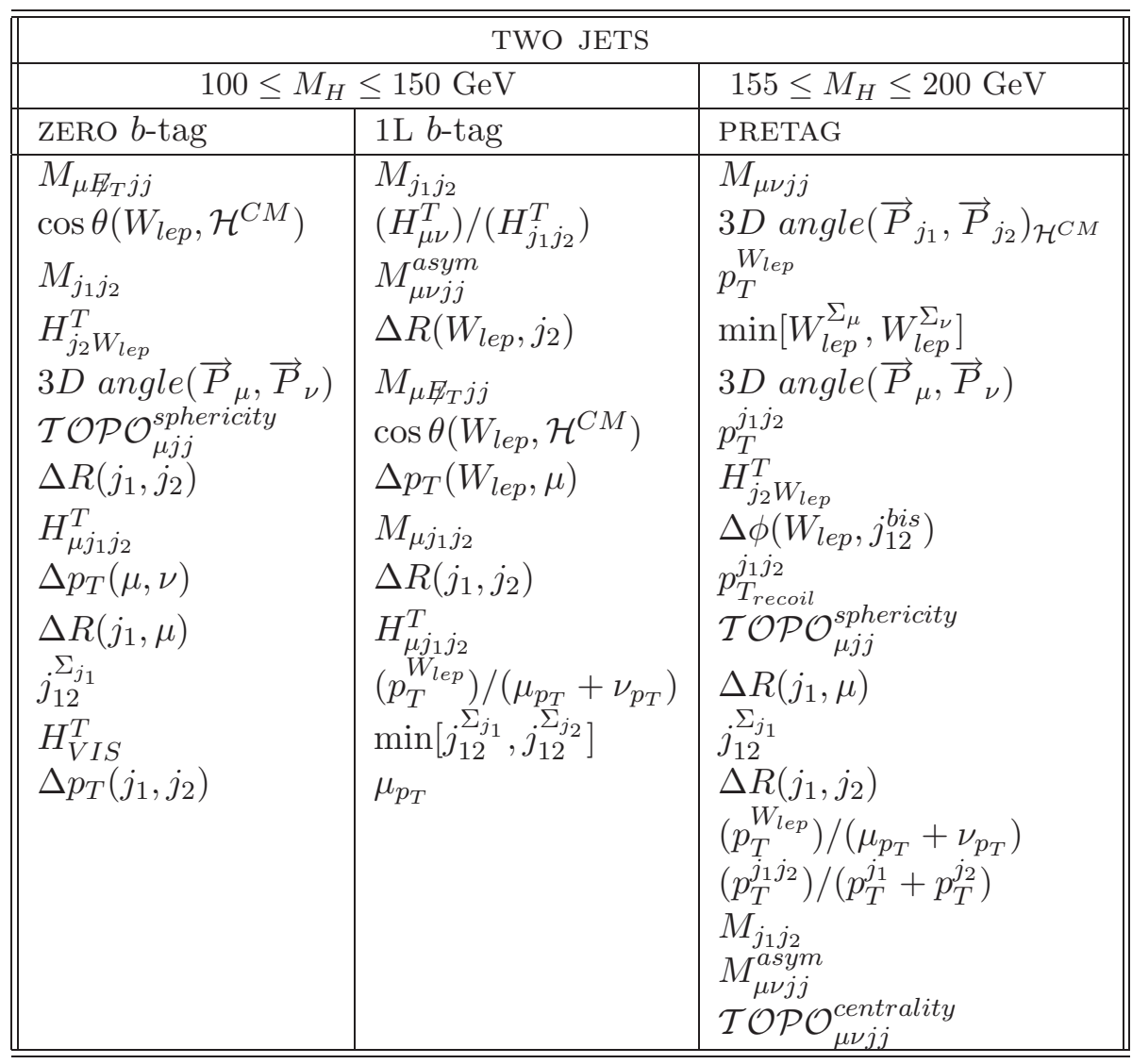

Table 7.1: List of MVA input variables used for each search in low mass $\left(100 \leq M_{H} \leq\right.$ $150 \mathrm{GeV})$ and high mass $\left(155 \leq M_{H} \leq 200 \mathrm{GeV}\right)$ regions for 2-jet events for each tagging category covered by the $H \rightarrow W W \rightarrow \mu \nu j j$ channel. Variables are ranked by importance. A description of all variables is found in Sec. 7.1.2. 


\begin{tabular}{|c|c|c|}
\hline \multicolumn{3}{|c|}{ THREE JETS } \\
\hline \multicolumn{2}{|c|}{$100 \leq M_{H} \leq 150 \mathrm{GeV}$} & $155 \leq M_{H} \leq 200 \mathrm{GeV}$ \\
\hline ZERO $b$-tag & $1 \mathrm{~L} b$-tag & PRETAG \\
\hline $\mathcal{F} \mathcal{W} \mathcal{M}_{j_{1} j_{2} j_{3}}^{l e p}$ & $\mathcal{F} \mathcal{W} \mathcal{M}_{j_{1} j_{2} j_{3}}^{l e p}$ & $\mathcal{F W} \mathcal{M}_{j_{1} j_{2} j_{3}}^{l e p}$ \\
\hline$M_{W_{l e p}}^{T}$ & $\mathcal{T O} \mathcal{O P O} \mathcal{O}_{\mu \nu j j}^{\text {centrality }}$ & $M_{\mu \nu j_{2}}$ \\
\hline$H_{\mu \nu j j}^{T^{\text {assym }}}$ & $M_{W_{l e p}}^{T}$ & $p_{T}^{W_{l e p}}$ \\
\hline$M_{\mu E_{T} j j}$ & $\cos \theta\left(W_{\text {lep }}, \mathcal{H}^{C M}\right)$ & $3 D$ angle $\left(\vec{P}_{\mu}, \vec{P}_{\nu}\right)$ \\
\hline$M_{j_{1} j_{2} \mu}$ & $\Delta \eta\left(j_{1}, \mu\right)$ & $M_{\mu \nu j j}$ \\
\hline $\mathcal{T O P} \mathcal{O}_{\mu \nu j j}^{\text {centrality }}$ & $M_{\mu j_{1} j_{2}}$ & $p_{T_{\text {recoil }}}^{j_{1} j_{2}}$ \\
\hline$\Delta \phi\left(W_{l e p}, j_{2}\right)$ & $\Delta R\left(W_{l e p}, \nu\right)$ & $3 D$ angle $\left(\vec{P}_{j_{1}}, \vec{P}_{j_{2}}\right)_{\mathcal{H}^{C M}}$ \\
\hline$M_{j_{1} j_{2} j_{3}}^{T}$ & $M_{\mu E_{T} j j}$ & $\left(p_{T}^{W_{l e p}}\right) /\left(\mu_{p_{T}}+\nu_{p_{T}}\right)$ \\
\hline$\Delta R\left(j_{1}, \mu\right)$ & $\Delta \phi\left(W_{l e p}, j_{2}\right)$ & $\Delta \phi\left(W_{l e p}, j_{12}^{b i s}\right)$ \\
\hline $\cos \theta\left(j_{1} j_{2}, \mathcal{H}^{C M}\right)$ & $\Delta \phi\left(j_{2} j_{3}, j_{3}\right)$ & $p_{T}^{j_{1} j_{2} j_{3}}$ \\
\hline $\mathcal{F} \mathcal{W} \mathcal{M}_{j_{1} j_{2} j_{3}}$ & $M_{j_{1} j_{2}}$ & $\mathcal{T} \mathcal{O P O} \mathcal{O}_{\mu \nu j j}^{\text {sphericity }}$ \\
\hline$\Delta \phi\left(j_{1}, j_{3}\right)$ & $M_{\mu \nu j j}^{a s y m}$ & $\mathcal{T O P} \mathcal{O}_{\mu \nu j j}^{\text {centrality }}$ \\
\hline$\left(p_{T}^{j_{2} j_{3}}\right) /\left(p_{T}^{j_{2}}+p_{T}^{j_{2}}\right)$ & $p_{T_{\text {recoil }}}^{W_{\text {lep }}}$ & $\Delta R\left(W_{l e p}, j_{1}\right)$ \\
\hline$\Delta \phi\left(W_{l e p}, j_{12}^{b i s}\right)$ & $\begin{array}{l}M_{j_{1} j_{2} j_{3}} \\
\mathcal{F} \mathcal{W} \mathcal{M}_{j_{1} j_{2} j_{3}}\end{array}$ & $\begin{array}{l}\Delta \phi\left(W_{l e p}, j_{2}\right) \\
\left(p_{T}^{j_{1} j_{2}}\right) /\left(p_{T}^{j_{1}}+p_{T}^{j_{2}}\right) \\
M_{j_{1} j_{2}} \\
\mathcal{F} \mathcal{W} \mathcal{M}_{j_{1} j_{2} j_{3}}\end{array}$ \\
\hline
\end{tabular}

Table 7.2: List of MVA input variables used for each search in low mass (100 $\leq M_{H} \leq$ $150 \mathrm{GeV})$ and high mass $\left(155 \leq M_{H} \leq 200 \mathrm{GeV}\right)$ regions for 3 -jet events for each tagging category covered by the $H \rightarrow W W \rightarrow \mu \nu j j$ channel. Variables are ranked by importance. A description of all variables is found in Sec. 7.1.2. 

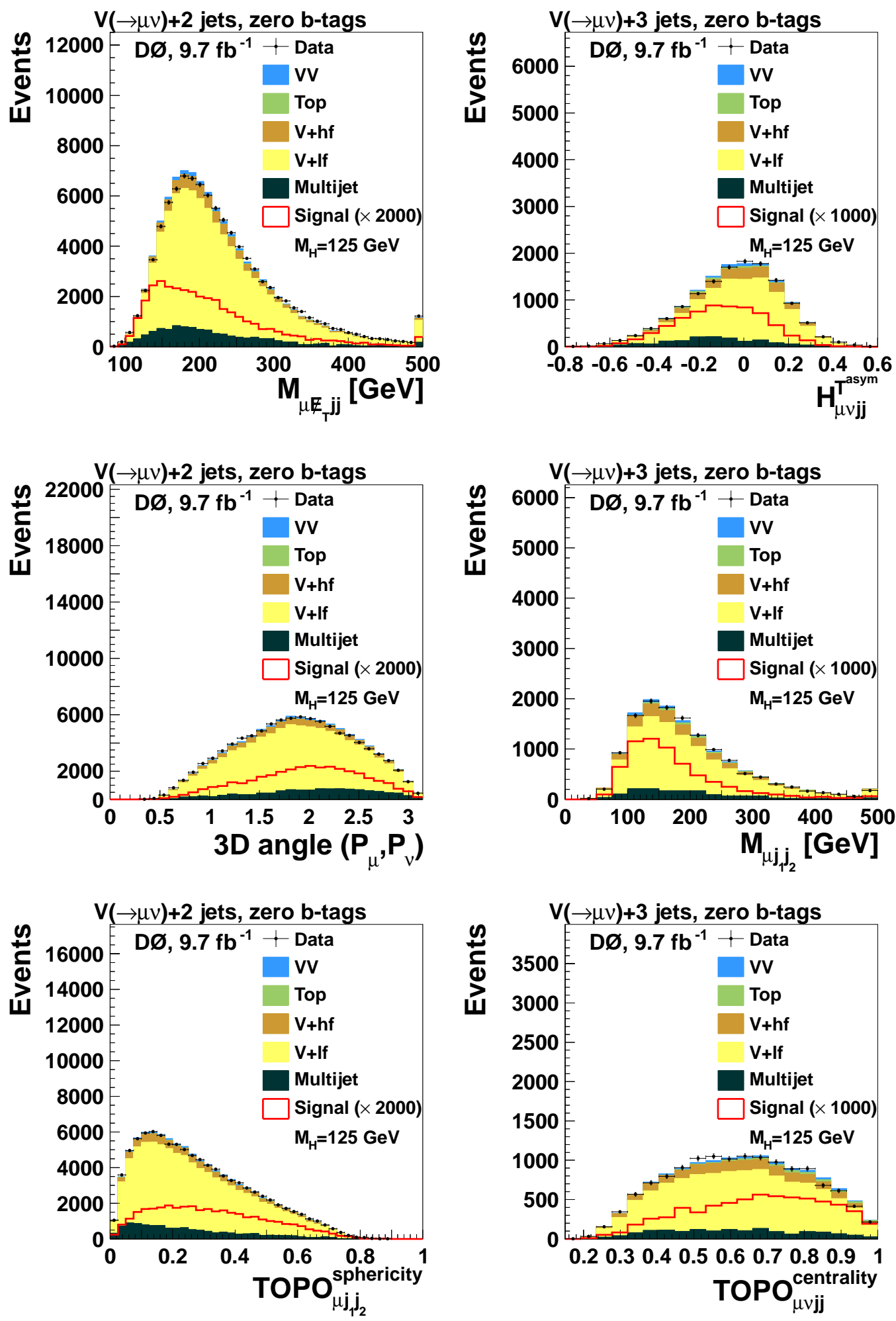

Figure 7.1: Distributions of MVA input variables used for the search in the low mass $\left(155 \leq M_{H} \leq 200 \mathrm{GeV}\right)$ region for all events in zero $b$-tag category that contain two jets (left) or three jets (right). The signal is shown for $M_{H}=125 \mathrm{GeV}$. Overflow events are added to the last bin in each histogram. 

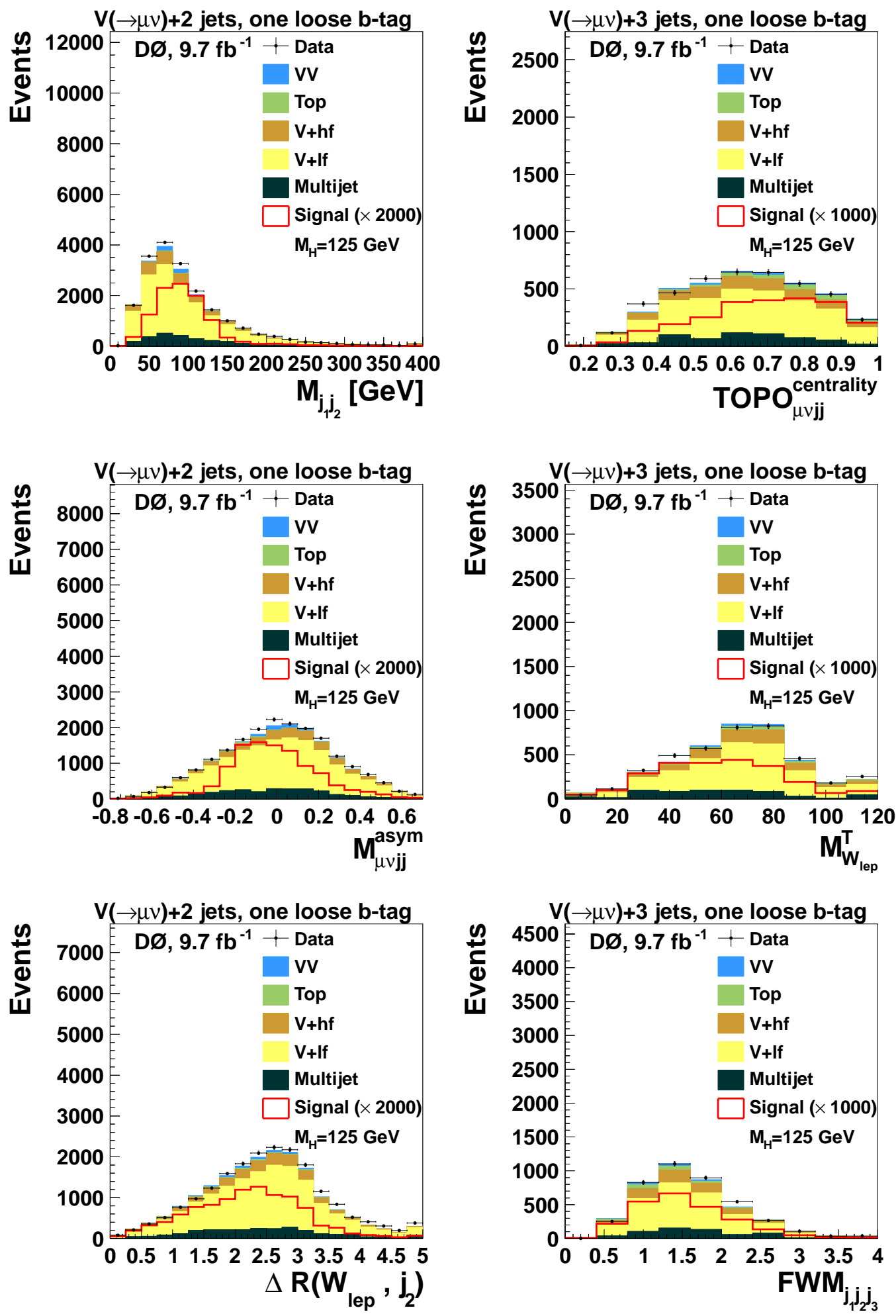

Figure 7.2: Distributions of MVA input variables used for the search in the low mass $\left(100 \leq M_{H} \leq 150 \mathrm{GeV}\right)$ region for all events in one-loose $b$-tag category that contain two jets (left) or three jets (right). The signal is shown for $M_{H}=125 \mathrm{GeV}$. Overflow events are added to the last bin in each histogram. 

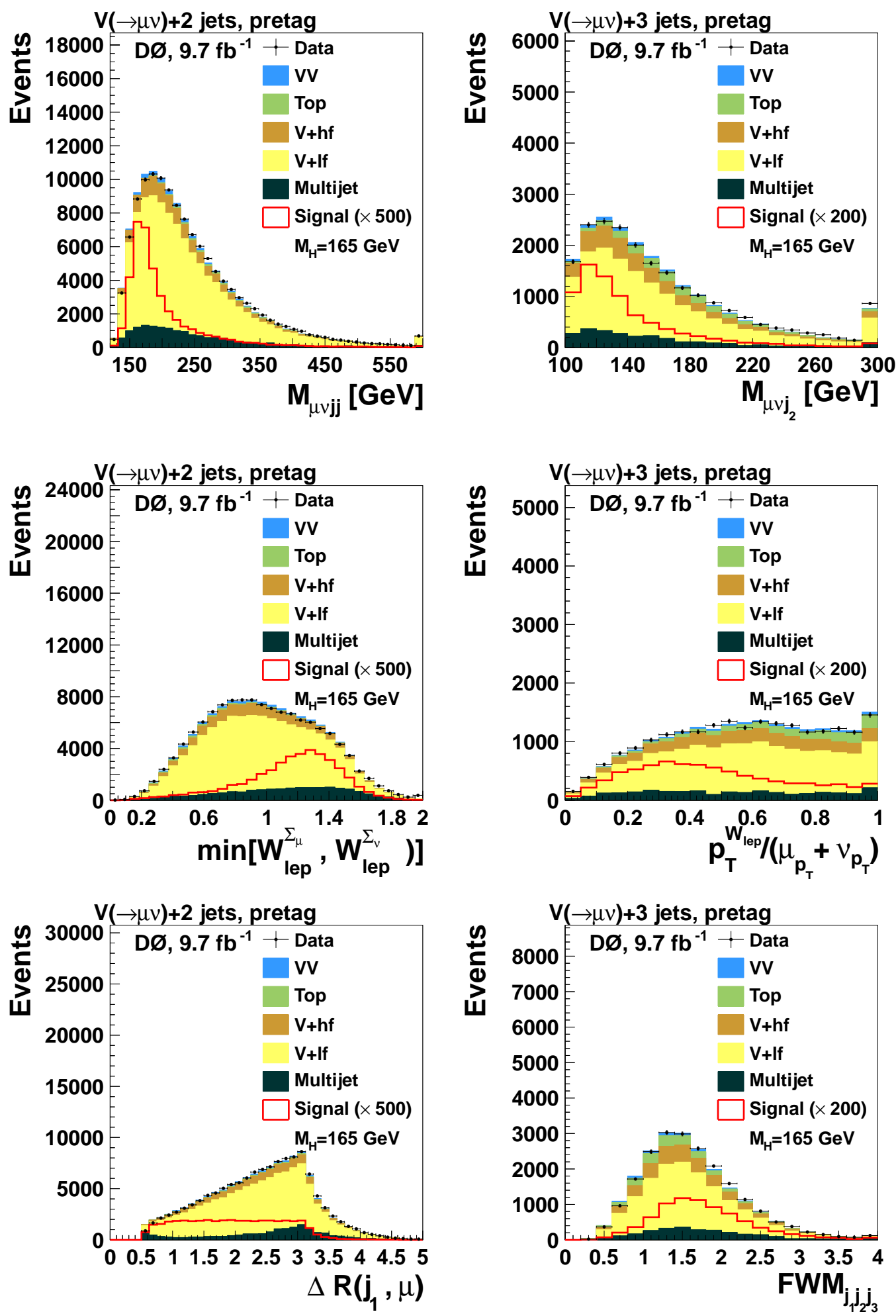

Figure 7.3: Distributions of MVA input variables used for the search in the high mass $\left(155 \leq M_{H} \leq 200 \mathrm{GeV}\right)$ region for all events containing two jets (left) or three jets (right). The signal is shown for $M_{H}=165 \mathrm{GeV}$. Overflow events are added to the last bin in each histogram. 


\section{Random forest parameters}

Besides the discriminating power, other important characteristics of the RF classifier include the stability of the RF performance and classification training and response time per event. These characteristics of the RF are optimized by minimizing the quadratic loss $\Lambda$ (Eqn. (7.1)) of the validation sample when varying the leaf size and the number of decision trees in the forest $[99,103]$. Details of the RF structure used in our analysis are listed in Table 7.3.

To support our parameter choices and to cross-check for overtraining (i.e. a focus on features that are too event specific during the training process), we examined the quadratic loss $\Lambda$ (Eqn. 7.1) in the validation sample as the number of trees used to build the RF increases. The study has been done for each jet multiplicity and each of zero tag and one loose tag channels at mass points $M_{H}=125 \mathrm{GeV}$ ) and for pretag channels at $M_{H}=$ $165 \mathrm{GeV}$. Figure 7.4 showing the change of the quadratic loss figure of merit (FOM) with the number of trees verifies that the effect of training is to asymptotically improved the performance of the RF classifier and no overtraining occurs.

Table 7.3: Parameters used for Random Forest training.

Number of trees in the forest: 25

Criterion for decision tree optimization: Gini index

Minimal number of events per terminal node: 100

Max number of variables used by each decision tree: 50

Number of input variables: varies between samples, see Tables 7.1-7.2

\subsubsection{Radom Forest Discriminants}

We evaluate the performance of each RF classifier on the corresponding testing sample and data set at each searched mass point within the range of $100 \leq M_{H} \leq 200 \mathrm{GeV}$.

Figures 7.5 and 7.6 show the RF discriminants for signal at $M_{H}=125 \mathrm{GeV}$ for the twoand three-jet channels in the zero $b$-tag and the one-loose $b$-tag categories, respectively. The $\mathrm{RF}$ discriminants for signal at $M_{H}=165 \mathrm{GeV}$ for all pretag events in the two- and three-jet channels are shown in Fig. 7.7. The distributions of RF discriminant for MC signals are likely to be shifted towards unity, while background events are likely to be shifted towards zero. Agreement is observed with expectations from SM background within the systematic uncertainty, and the distributions of the RF discriminants are therefore used to set upper limits on the cross section for SM Higgs boson production as presented in Sec. 9.2. 

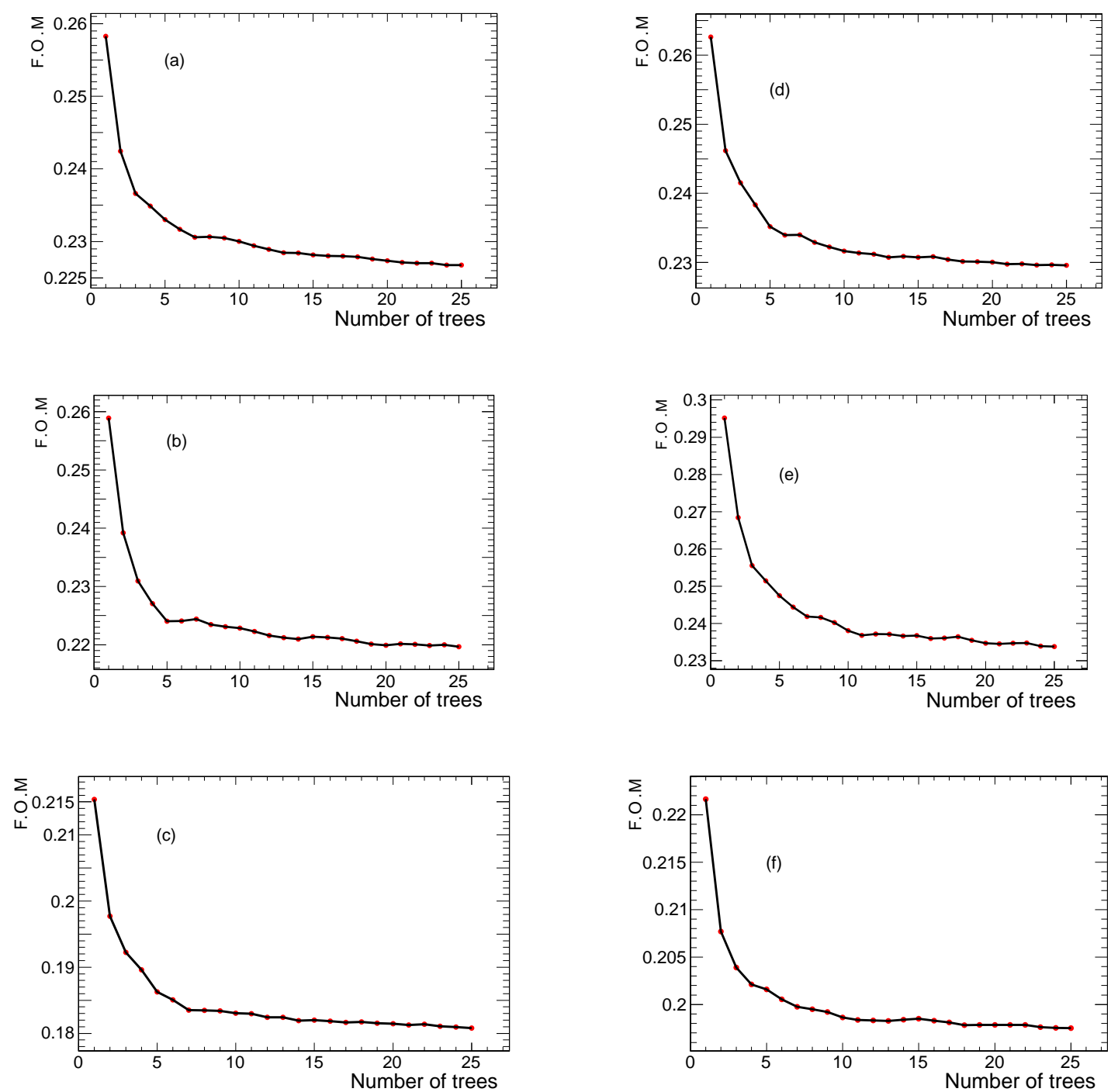

Figure 7.4: Examination of the quadratic loss F.O.M. when varying the number of trees used for RF training in two-jet zero tag (a), two-jet one loose tag (b), two-jet pretag (c), three-jet zero tag (d), three-jet one loose tag (e) and three-jet pretag (f) sub-channels. The zero-tag and one loose tag channels are trained at $M_{H}=125 \mathrm{GeV}$ and the pretag channels are train at $M_{H}=165 \mathrm{GeV}$. No indication of overtraining is observed. 

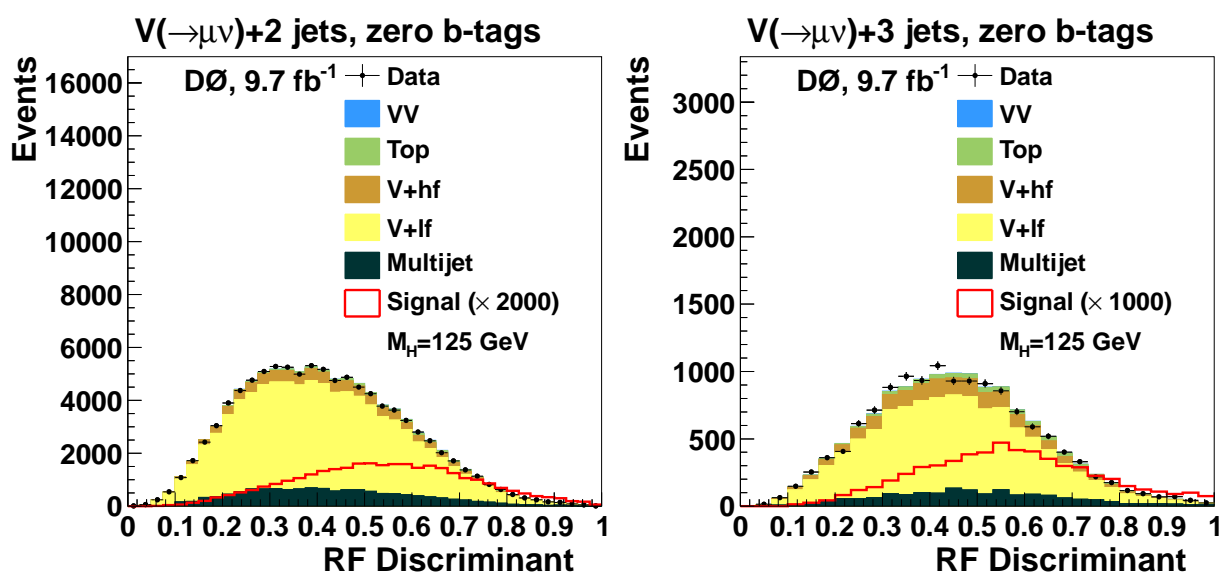

Figure 7.5: Distributions of RF discriminant for signal at $M_{H}=125 \mathrm{GeV}$ in zero $b$-tag events with exactly two jets (left) or three jets (right)
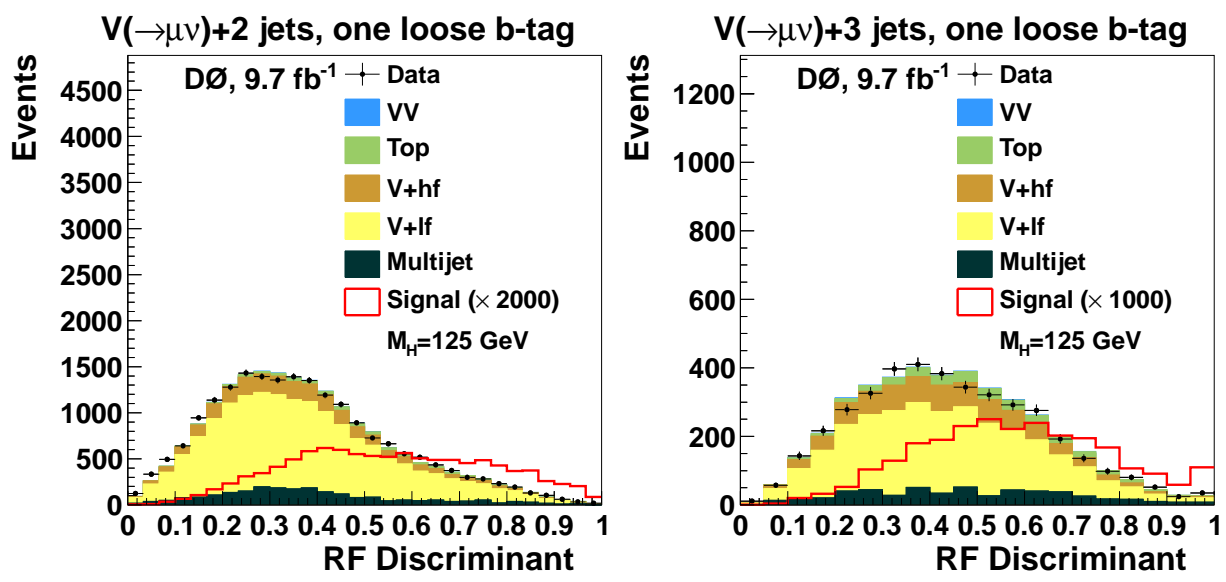

Figure 7.6: Distributions of RF discriminant for signal at $M_{H}=125 \mathrm{GeV}$ in one-loose $b$-tag events with exactly two jets (left) or three jets (right)
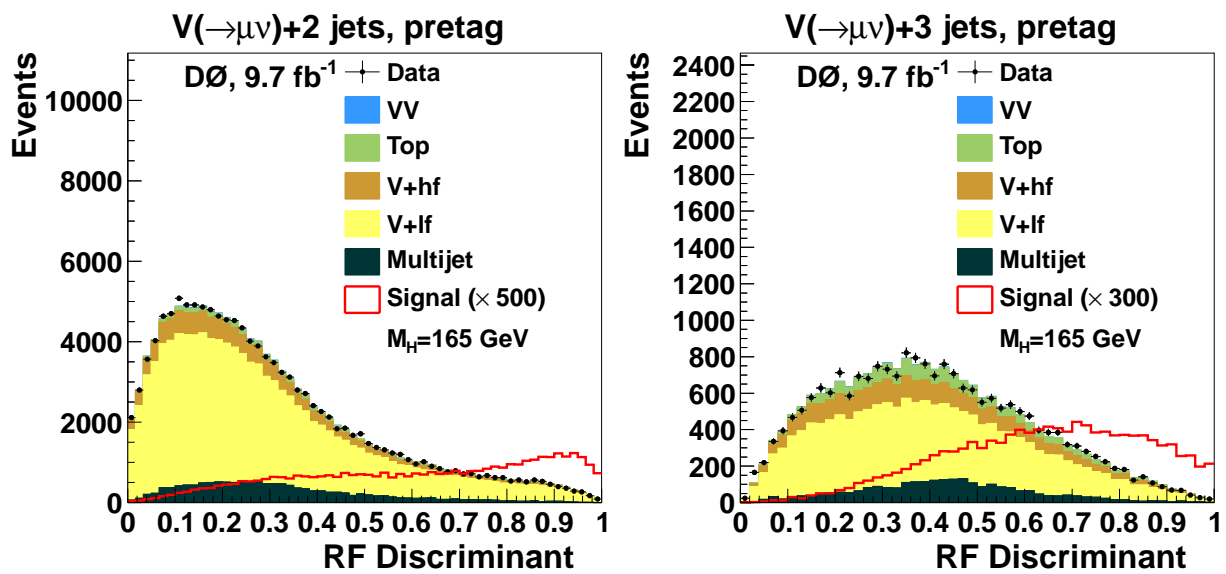

Figure 7.7: Distributions of $\mathrm{RF}$ discriminant for signal at $M_{H}=165 \mathrm{GeV}$ in all pretag events with exactly two jets (left) or three jets (right) 


\subsection{MVA Analysis in $Z H \rightarrow \ell \ell b b$ Search}

\subsubsection{Matrix Elements Discriminants}

The Matrix Element technique makes an optimal use of available kinematic information in building a discriminant to separate signal from backgrounds. In this approach, the ME probabilities for each event to originate from a certain process are evaluated based on the parton-level differential cross-section and a parameterized detector response. The probabilities for individual processes are then combined to form a ME discriminant that is used to characterize events as background-like or signal-like. The ME discriminant is constructed such that signal-like events are most likely to take values close to unity, while background-like events are likely to pile up near zero.

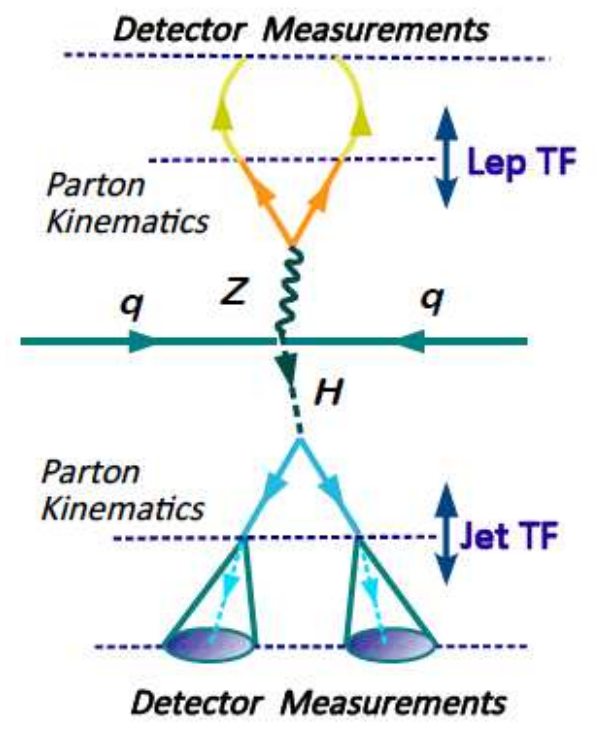

Figure 7.8: Schematic of the Matrix Element modelling for $Z H \rightarrow \ell \ell b b$. Transfer functions (TF) are used to map calculated parton states to detector level observables.

\section{The Matrix Element Probabilities}

A measured event $s$ observed at detector level is the result from an underlying process with parton kinematics $\boldsymbol{k}$ (Fig.7.8). The ME probability $p(\mathbf{s})$ for the event $\boldsymbol{s}$ being from a specific signal or background process is calculated by integrating over the phase space $\Phi(\mathbf{k})$ of outgoing partons, weighing each configuration by the matrix element probability $\mathcal{M}$ for the process to produce a final state with kinematics $\boldsymbol{k}$. A transfer function $\boldsymbol{T}(\boldsymbol{k}, s)$ describes the probability for the final state partons of the event $\boldsymbol{k}$ to produce a detector measurement $s$ :

$$
p(\mathbf{s})=\int|\mathcal{M}(\mathbf{k})|^{2} \mathbf{T}(\mathbf{k}, \mathbf{s}) d \Phi(\mathbf{k})
$$


Taking $m_{1}, m_{2}, q_{1}$ and $q_{2}$ as the masses and the four momenta of the incident particles, we have:

$$
d \Phi(\mathbf{k})=\frac{2 \pi^{4}}{4 \sqrt{\left(q_{1} q_{2}\right)^{2}-m_{q_{1}}^{2} m_{q_{2}}^{2}}} \delta^{4}\left(q_{1}+q_{2}-\sum_{i=1}^{n} p_{i}\right) \prod_{i=1}^{n} \frac{d^{3} p_{i}}{(2 \pi)^{3} 2 E_{i}},
$$

where $\boldsymbol{k}$ comprises the four-vector states of the outgoing partons. Since the reaction is initiated by partons inside the proton and antiproton, the phase space has to be convoluted with the parton distribution functions:

$$
d \Phi(\mathbf{k})=\frac{2 \pi^{4}}{4 \sqrt{\left(q_{1} q_{2}\right)^{2}-m_{q_{1}}^{2} m_{q_{2}}^{2}}} \delta^{4}\left(q_{1}+q_{2}-\sum_{i=1}^{n} p_{i}\right) \prod_{i=1}^{n} \frac{d^{3} p_{i}}{(2 \pi)^{3} 2 E_{i}} f\left(q_{1}\right) d q_{1} f\left(q_{2}\right) d q_{2},
$$

where $f\left(q_{i}\right) d q_{i}$ is the probability that a parton $i$ carries a longitudinal momentum between $q_{i}$ and $q_{i}+d q_{i}$.

The whole-event transfer function $\boldsymbol{T}(\boldsymbol{k}, \boldsymbol{s})$ is factored into the detector response for each individual particle species in the final state, jet transfer functions for quarks and muon or electron transfer functions for leptons:

$$
\mathbf{T}(\mathbf{k}, \mathbf{s})=\prod_{i=q u a r k s, \text { leptons }} T_{i}\left(k_{i}, s_{i}\right)
$$

The matrix elements $\left(|M(\mathbf{k})|^{2}\right)$ are calculated using MCFM [75], a general purpose MC event generator. We perform the calculation to leading order in perturbation theory where the final state of the $\ell \ell b b$ system has zero transverse momentum. To incorporate the effects of additional processes such as initial or final state radiation, we fit a prior $p_{T}$ distribution from the fully simulated MC events (which includes additional processes that boost the $\ell \ell b b$ system) and boost the matrix element in the frame with no net $p_{T}$ to replicate this prior $p_{T}$ distribution $\left(p\left(p_{T}\right)\right)$. The full ME is approximately factorized as:

$$
\left|\mathcal{M}\left(p_{\mathrm{T}} \neq 0\right)\right|^{2}=\left|\mathcal{M}\left(p_{\mathrm{T}}=0\right)\right|^{2} \times p\left(p_{\mathrm{T}}\right) .
$$

Computational efficiency is achieved by restricting the integral over underlying parton kinematics $d \mathbf{k}$ to a region nearby the measured event $\boldsymbol{s}$.

\section{Transfer Functions}

The transfer function $T\left(x_{\text {parton }}, x_{\text {reco }}\right)$ is a parameterized response function of the detector to a parton (quark or lepton), and it represents the probability that a process with kinematic quantity $x_{\text {parton }}$ at the parton level will produce a detector measurement $x_{\text {reco }}$. The experimental measurement of the $\ell \ell b b$ final state is primarily affected by energy measurement and resolution effect, and we can assume that the reconstructed jet and muon directions describe the parton directions accurately. The jet (electron) transfer function 
relates the measured jet (electron) energy to the parton quark (electron) energy, while the muon transfer function relates the observed muon momentum to its incident momentum.

\section{- Jet Transfer Functions}

The jet transfer function relating the reconstructed jet energy to the corresponding quark energy at parton level is parameterized based on the shape of the fractional energy resolution distribution:

$$
x=\frac{E_{\text {part }}-E_{\text {reco }}}{E_{\text {part }}} .
$$

In order to model the central peak and the asymmetric tails of the distribution, we use a double Gaussian parameterization and add a small flat background to prevent rare outliers from distorting the fit. The jet transfer function is parameterized as:

$\mathcal{F}(x)=\frac{1}{\sqrt{2 \pi} \sigma_{a}} \alpha(1-\gamma) \exp \left\{-\frac{\left(x-\mu_{a}\right)^{2}}{2 \sigma_{a}^{2}}\right\}+\frac{1}{\sqrt{2 \pi} \sigma_{b}} \beta(1-\gamma) \exp \left\{-\frac{\left(x-\mu_{b}\right)^{2}}{2 \sigma_{b}^{2}}\right\}+\frac{\gamma}{2},(7$.

where parameters of the two Gaussians are constrained as:

$$
\begin{array}{rlrl}
\mu_{a} & =\left(p_{1}+\frac{p_{2}}{E_{\text {part }}}\right)-p_{5} \beta, & \sigma_{a}=\left(p_{3}+\frac{p_{4}}{E_{\text {part }}}\right) \sqrt{1+\frac{p_{6}}{1+p_{7}}}, & \alpha=\frac{1}{1+p_{6}}, \\
\mu_{b}=\left(p_{1}+\frac{p_{2}}{E_{\text {part }}}\right)+p_{5} \alpha, & \sigma_{b}=\left(p_{3}+\frac{p_{4}}{E_{\text {part }}}\right) \sqrt{\frac{p_{7}}{1+p_{7}}}, \quad \beta=\frac{p_{6}}{1+p_{6}} .
\end{array}
$$

The data for the fit is obtained from fully simulated MC events where parton-level quarks are matched to reconstructed jets within a sufficiently small angular range. The fitted data are selected based on quark flavors and jet decay products. We perform the fit for the transfer function separately for light flavor jets and b-jets originating from the parent quark decaying with and without a muon. For each type of jet, we derived different parameterizations for three $\eta$ regions in the detector: $\left|\eta_{1}\right|<0.8$, $0.8<\left|\eta_{2}\right|<1.6$, and $1.6<\left|\eta_{3}\right|<2.5$. The jet transfer function for jets in the $\left|\eta_{1}\right|$ region is shown in Fig. 7.9, and the $|\eta|$ dependencies of the jet transfer functions for the three types of jets are shown in Figs. 7.10, 7.11, and 7.12.

We perform closure tests to compare the reconstructed energy and transverse momentum of $b$-jets in the full MC, to those generated from the parton $b$ quarks after application of the transfer functions, as shown in Fig. 7.13.

\section{- Lepton transfer function}

The enery of electrons is measured by the EM calorimeter, therefore, the electron transfer function is parameterized similarly to the jet transfer function. The muon momentum is obtained from the track curvature measurement, making the inverse transverse momentum $\left(1 / p_{\mathrm{T}}\right)$ best suited for a Gaussian parameterization. We use 


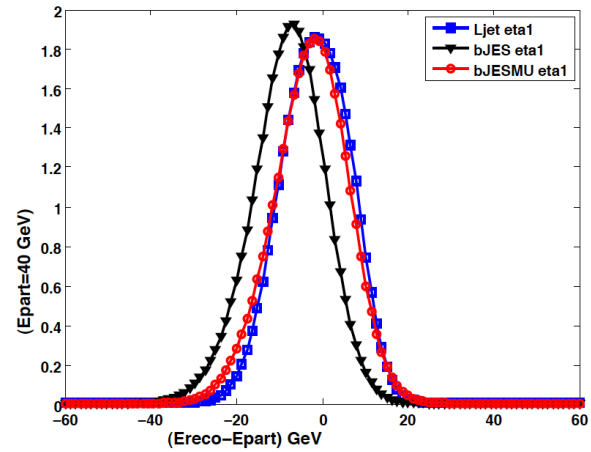

Figure 7.9: The transfer function for light jets (red), $b$-jets with $\mu$ (blue) and $b$-jets without $\mu$ (black) in $\left|\eta_{1}\right|$ region

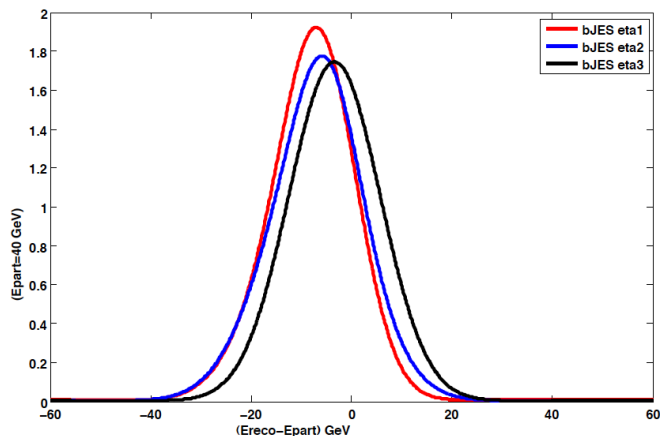

Figure 7.11: The transfer function for $b$ jets decaying without a muon in three $\eta$ regions

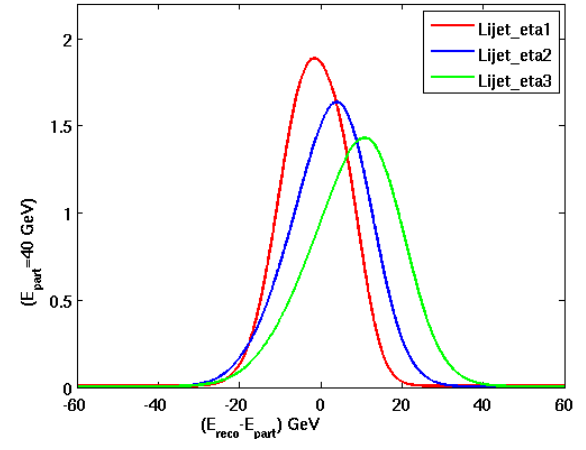

Figure 7.10: The transfer function for light jets in three $\eta$ regions

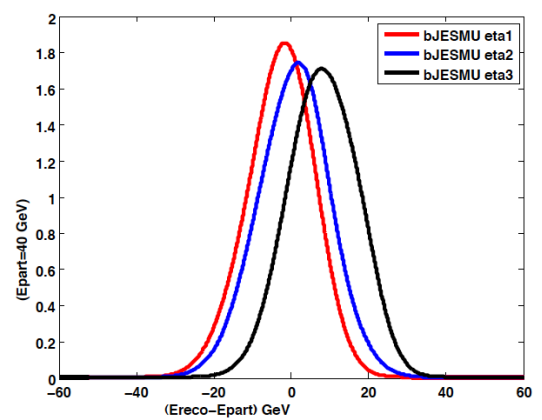

Figure 7.12: The transfer function for $b$ jets decaying with a muon in three $\eta$ regions

the same orthogonal double Gaussian parameterization as for the jet transfer function(Eqn. (7.3)), with the substitution $E \rightarrow 1 / p_{\mathrm{T}}$. The closure tests comparing the reconstructed muon energies and transverse momentum in the full MC to those generated from the parton-level muon after application of the transfer functions is shown in Fig. 7.14.

\section{Prior $p_{\mathrm{T}}$}

We use a triple Gaussian fitting function to parameterize the transverse momentum $p_{T}$ of the $l l b b$ system:

$$
\mathcal{F}_{p_{T}}(x)=f_{0}+f_{1} \cdot G\left(x ; \sigma_{1}, x_{1}\right)+f_{2} \cdot G\left(x ; \sigma_{2}, x_{2}\right)+f_{3} \cdot G\left(x ; \sigma_{3}, x_{3}\right),
$$

where $x$ presents the prior $p_{T}$ of the $l l b b$ system, $x_{i}$ and $\sigma_{i}$ are the mean and sigma of the $i$ th Gaussian. The fit is performed using the parton shower MC generated by PYTHIA, which models additional processes that boost the $l l b b$ system. Closure tests for the prior $p_{\mathrm{T}}$ distribution are shown in Fig. 7.15. 

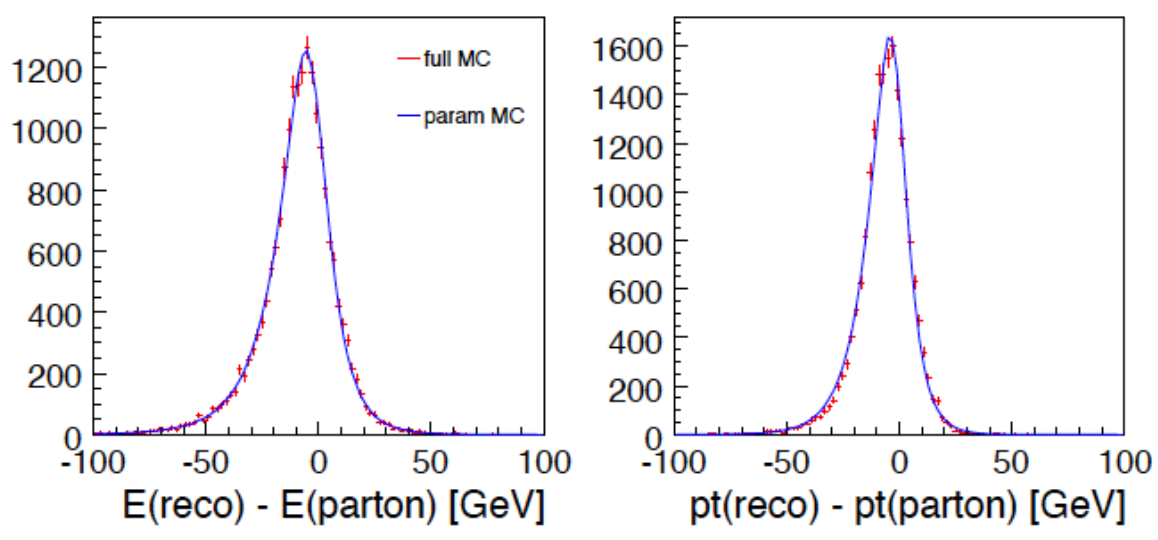

Figure 7.13: Energy and $p_{\mathrm{T}}$ closure test for $b$ jet transfer functions.
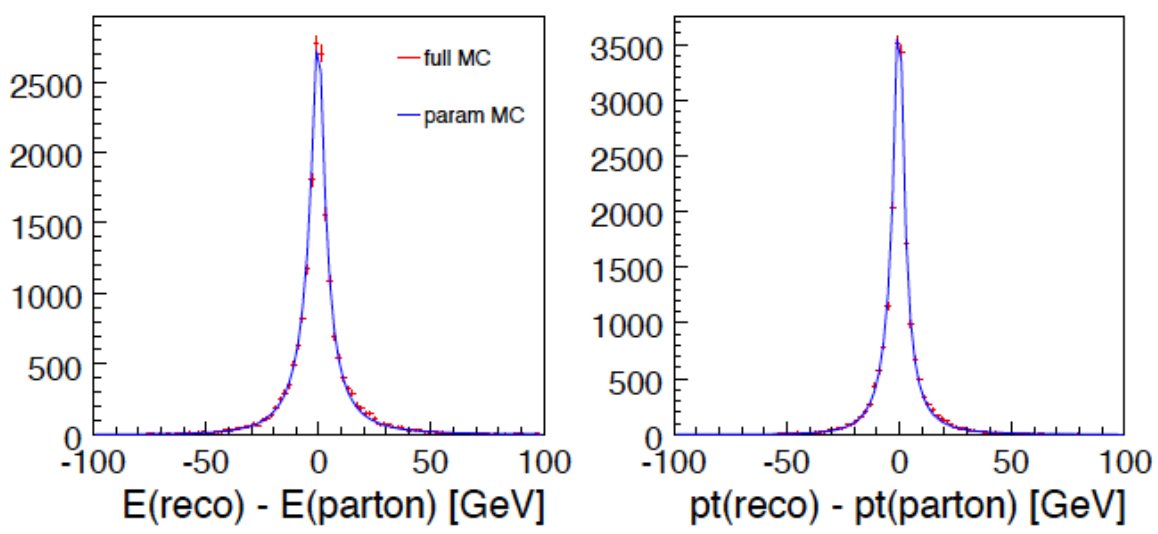

Figure 7.14: Energy and $p_{\mathrm{T}}$ closure test for muon transfer function.
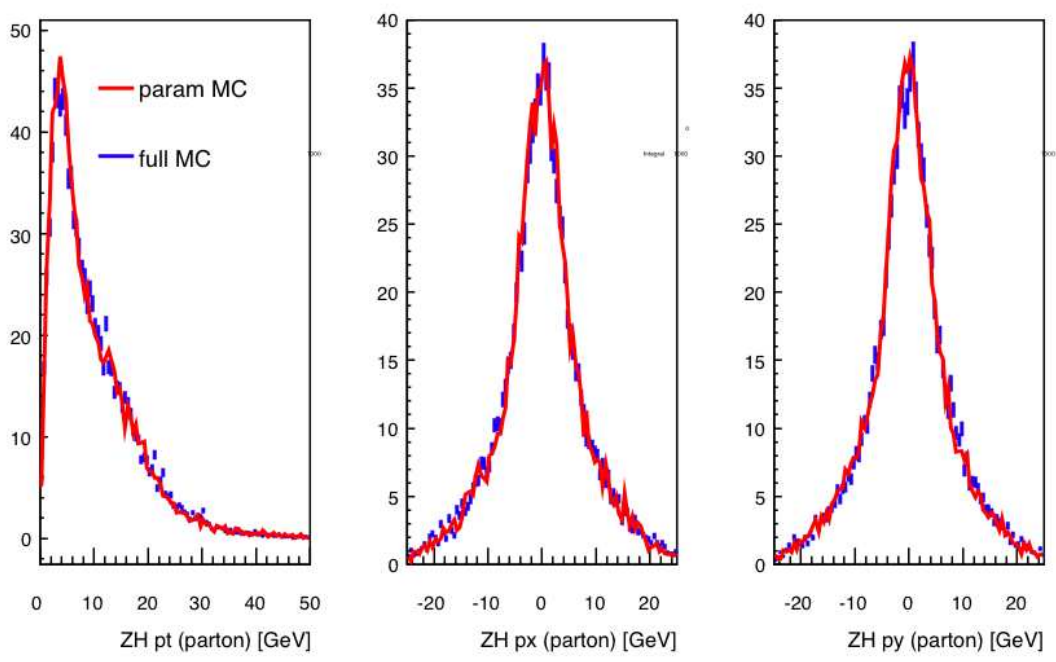

Figure 7.15: $p_{x}, p_{y}$ and $p_{\mathrm{T}}$ closure test for $Z H \rightarrow \ell \ell b b$ prior $p_{\mathrm{T}}$. 


\section{Matrix Element Disciminant}

The ME probabilities for each measured event being from either signal or background processes are calculated and combined to construct a discriminant:

$$
D=\frac{P_{Z H}}{P_{Z H}+\alpha \cdot P_{Z j j}+\beta \cdot P_{Z b b}},
$$

where $P_{Z H}, P_{Z j j}$ and $P_{Z b b}$ are ME probabilities for the measured event $(s)$ observed at the detector to be from the signal $Z H \rightarrow \ell \ell b b$ and background $Z+L F, Z+b b$ processes, respectively.

The normalization parameters $\alpha$ and $\beta$ are obtained by maximizing the unbinned likelihood

$$
\log (L)=\sum_{b k g^{[i]}} \frac{w_{b k g}^{[i]}}{W_{b k g}^{\text {total }}} \log \left(1-D^{[i]}\right)+\sum_{\text {sig }} \frac{w_{\text {sid }}^{[i]}}{W_{\text {sig }}^{\text {total }}} \log \left(D^{[i]}\right),
$$

where $w^{[i]}$ is the weight of signal or background $\mathrm{MC}$ events and the sum is over events in the training $\mathrm{MC}$ sample. A comparison of the matrix-element discriminant for background and signal in a sample of test events with two-loose $b$-tags in the muon channel is shown in Fig. 7.16 for a hypothesized SM Higgs boson mass of $M_{H}=125 \mathrm{GeV}$. Similar distributions have been calculated for Higgs masses ranging from 100 to $150 \mathrm{GeV}$ in $5 \mathrm{GeV}$ increments. The constructed ME discriminants will later be used as inputs for the procedure of setting upper limits on the SM Higgs boson production, and then the resulting limits will be compared to that obtained using the $\mathrm{RF}$ discriminants to validate the $\mathrm{RF}$ technique (Sec. 9.3.2).
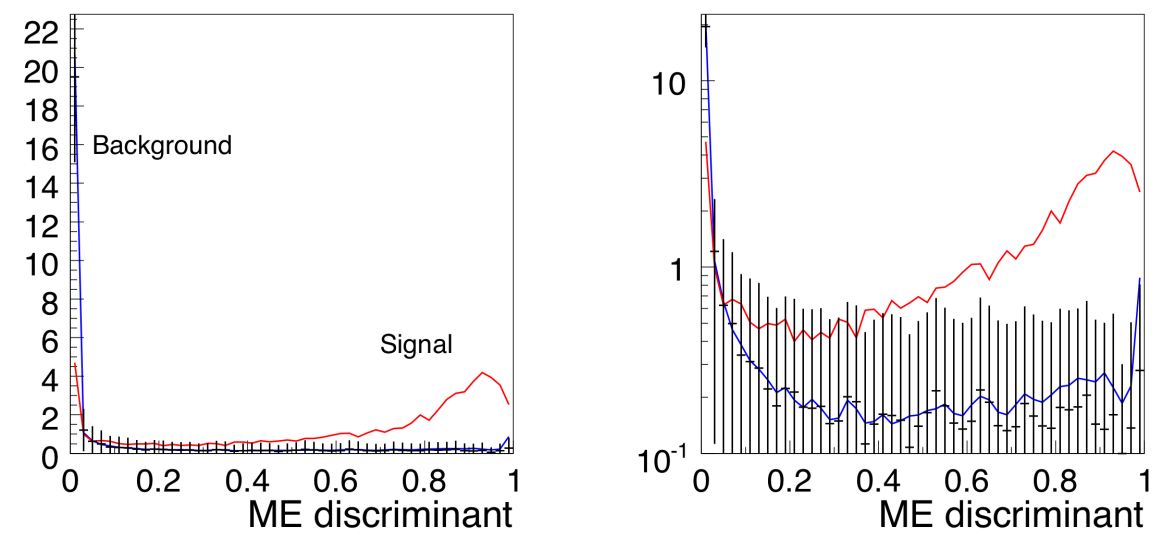

Figure 7.16: Output of the ME discriminant for signal (red), background (blue) and data (black), shown in linear (left) and log scales (right). 


\subsubsection{Kinematic Fit and Random Forest Discriminants}

For the machine learning approach in the $Z H \rightarrow l l b b$ analysis, we apply the multivariate analysis strategy based on the random forest of decision trees implemented in the TMVA package to improve the separation of signal from background. We first employ a kinematic fit to improve resolution of the reconstructed invariant mass $M_{j j}$ of the two leading jets in the final state, the most important input variable for the $\mathrm{RF}$ in the $Z H \rightarrow l l b b$ analysis [104, 11]. We then use the RF classifier to provide the discriminant distributions for the final statistical analysis, applied in a two-step process [11]. At the first step, the events are divided into independent $t \bar{t}$-depleted and $t \bar{t}$-enriched subchannels using a dedicated $R F$ that is trained to discriminate signal from the $t \bar{t}$ background. At the second step, final discriminants are constructed to separate signal from all backgrounds for each of the $t \bar{t}$-depleted and $t \bar{t}$-enriched samples.

\section{Kinematic Fit}

To make optimal use of the available kinematic information, the energies of the two leptons that form the $Z(\rightarrow l l)$ candidate and the two jets that form the Higgs candidate $H(\rightarrow b b)$ are fit within their resolutions to values which maximize a likelihood function:

$$
\mathcal{L}=\prod_{i} f\left(y_{i}^{\text {obs }}, y_{i}^{\text {pred }}\right)
$$

where $f\left(y_{i}^{o b s}, y_{i}^{\text {pred }}\right)$ is the probability density for observing $y_{i}^{\text {obs }}$ when the predicted value is $y_{i}^{\text {pred }}$. The transfer functions derived in Sec. 7.2.1 are used as probability densities for the energy resolution of jets or electrons and for the inverse transverse momentum $\left(1 / p_{T}\right)$ for muon. We use three different sets of jet transfer functions, (i) jets that originate from a b quark and do not contain a muon, (ii) jets that originate from a b quark and contain a muon, and (iii) jets that originate from a light quark or gluon. Each set includes three parameterized functions for different $\eta$ regions in the detector.

The kinematic fit is subjected to the following three constraints:

- The reconstructed dilepton mass must be consistent with the $Z$ boson mass $\left(M_{\ell \ell}=\right.$ $\left.M_{Z}\right)$ is characterized by a Breit-Wigner distribution with width $\Gamma_{Z}=2.4952 \mathrm{GeV}$ [105].

- Each of the $x$ and $y$ components of the vector sum of the transverse momenta of all leptons and jets in the final state, which allows for a $Z+$ jets system boost along the $x$ or $y$ axis, must be consistent with zero in Gaussian distributions with the width of $7 \mathrm{GeV}$ (the width is determined from the simulated $\mathrm{ZH}$ samples).

The constraints on the fit are implemented as probability densities for kinematic constraints 


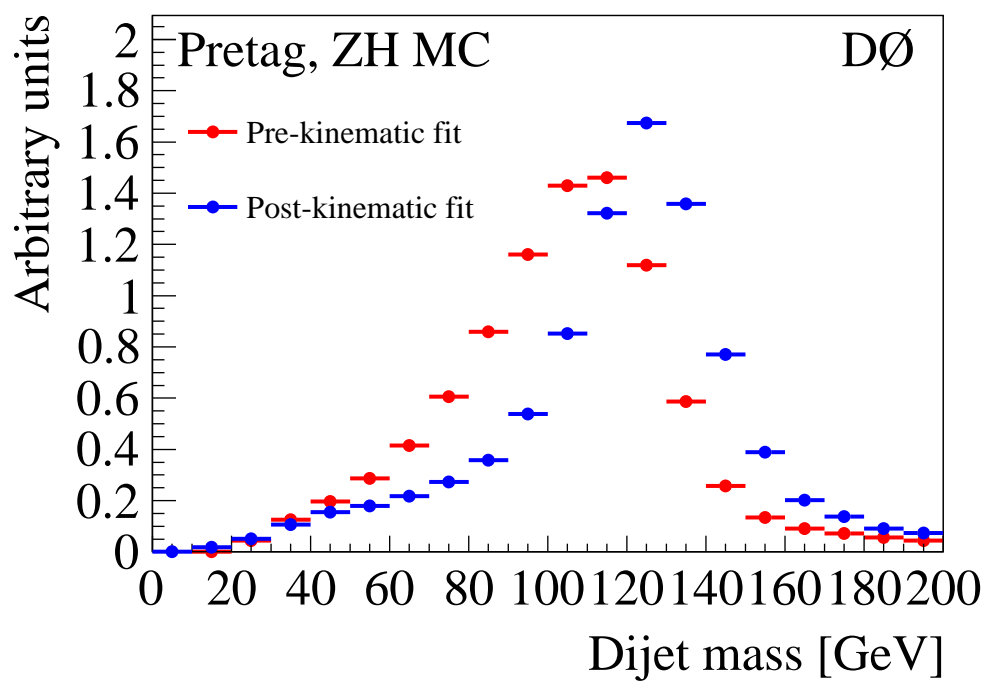

Figure 7.17: The dijet invariant mass for the simulated $Z H$ signal, at $M_{H}=125 \mathrm{GeV}$, summed over all lepton channels in the pretag sample, shown before and after the kinematic fit.

$C_{j}$ that are multiplied with the likelihood function:

$$
\mathcal{L}=\prod_{i} f\left(y_{i}^{\text {obs }}, y_{i}^{\text {pred }}\right) \cdot \prod_{j=1,2,3} C_{j}
$$

where

$$
\begin{array}{r}
C_{1} \equiv C_{M_{Z}}=\frac{1}{\left(M_{l l}^{2}-M_{Z}^{2}\right)^{2}+M_{Z}^{2} \Gamma_{Z}^{2}}, \\
C_{2} \equiv C_{p_{\mathrm{x}}}=\frac{1}{\sqrt{2 \pi} \sigma_{x}} \exp \left\{-\frac{\left(p_{\mathrm{x}}^{o b s}-p_{\mathrm{x}}^{p r e d}\right)^{2}}{2 \sigma_{x}^{2}}\right\} \\
C_{3} \equiv C_{p_{\mathrm{y}}}=\frac{1}{\sqrt{2 \pi} \sigma_{y}} \exp \left\{-\frac{\left(p_{\mathrm{y}}^{\text {obs }}-p_{\mathrm{y}}^{\text {pred }}\right)^{2}}{2 \sigma_{y}^{2}}\right\} .
\end{array}
$$

The fit to maximize the likelihood in Eqn. (7.5) is equivalent to the fit to minimize the negative logarithm of the likelihood :

$$
-\ln \mathcal{L}=-\sum_{i} \ln f\left(y_{i}^{\text {obs }}, y_{i}^{\text {pred }}\right)-\sum_{j} \ln C_{j}
$$

The kinematic fit corrects the measured jet energies to their best fit values and improves the dijet mass resolution by $10-15 \%$, depending on $M_{H}$. Distributions of the dijet invariant mass spectra, before and after adjustment by the kinematic fit, are shown in Fig. 7.17. The resolution for $M_{H}=125 \mathrm{GeV}$ is $\approx 15 \mathrm{GeV}$ after the fit. 


\section{Random Forest Discriminant}

We use a two-stage random forest training to improve the discrimination of signal from background $[11,106]$. In the first stage, we train a dedicated $\mathrm{RF}(t \bar{t} \mathrm{RF})$ that considers $t \bar{t}$ as the only background and $Z H$ as the signal. This approach takes advantage of the distinctive signature of the $t \bar{t}$ background, for instance the presence of large $E_{T}$. We use the $t \bar{t} \mathrm{RF}$ output to define two independent regions, a $t \bar{t}$-enriched region $(t \bar{t} \mathrm{RF}<0.5)$ and a $t \bar{t}$-depleted region $(t \bar{t} \mathrm{RF}>=0.5)$, for each of the single $b$-tag (ST) and double $b$-tag (DT) categories (Fig. 7.18).
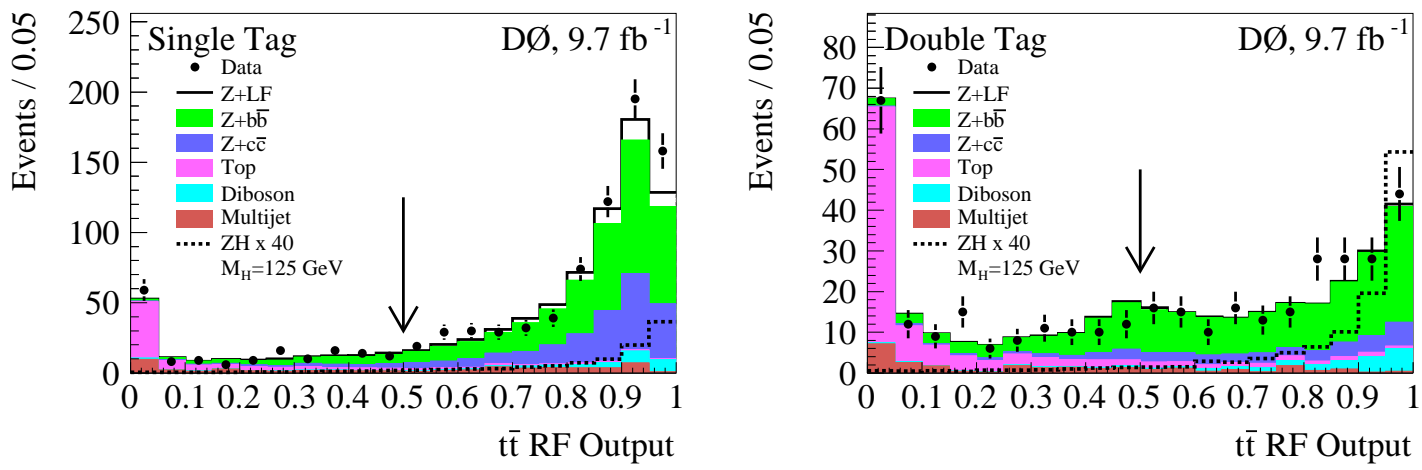

Figure 7.18: The $t \bar{t} \mathrm{RF}$ output for all lepton channels combined in ST and DT events trained for $M_{H}=125 \mathrm{GeV}$. The signal distributions are scaled by a factor of 40 . The arrows indicate the $t \bar{t} \mathrm{RF}$ selection requirement used to define the $t \bar{t}$-enriched and $t \bar{t}$-depleted subsamples.

In the second stage, we train a global RF to separate the $Z H$ signal from all backgrounds for each of the $t \bar{t}$-enriched and $t \bar{t}$-depleted regions. We consider ST and DT events separately and train the discriminants for each mass point in the range $90 \leq M_{H} \leq 150 \mathrm{GeV}$ in steps of $5 \mathrm{GeV}$. The input variables selected for the RF include particle 4 -vectors, angles between objects in the final state, and combinations of kinematic variables such as reconstructed masses and event shapes. Among them, the reconstructed invariant mass of the two $b$-jet candidates after the jet energies are adjusted by the kinematic fit is the most powerful variable in discriminating signal from background.

Figures 7.2.2 and 7.20 show the distributions of the global RF discriminants trained for $M_{H}=125 \mathrm{GeV}$ for ST and DT events in the $t \bar{t}$-enriched and $t \bar{t}$-depleted regions, respectively. Discrepancies between the distributions of data and the background prediction are covered by systematic uncertainties, and the global RF discriminants are used to extract results for the production of a SM Higgs boson. 

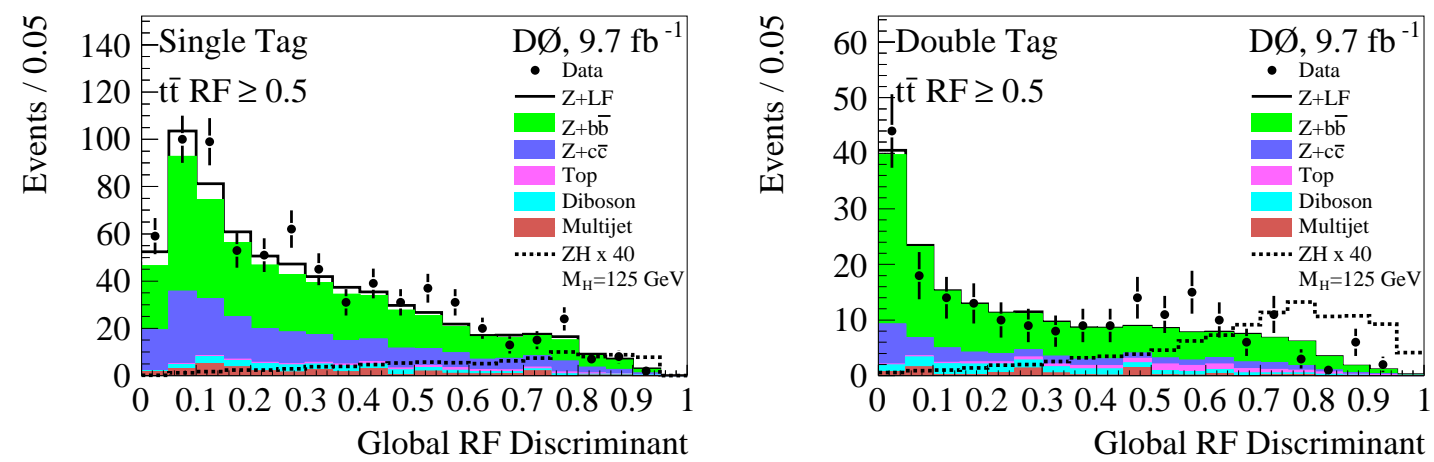

Figure 7.19: Distributions of Global RF discriminants for ST and DT events in the $t \bar{t}$ enriched region for $M_{H}=125 \mathrm{GeV}$. The signal distributions correspond to the $M_{H}$ used for the RF training and are scaled by a factor of 40 .
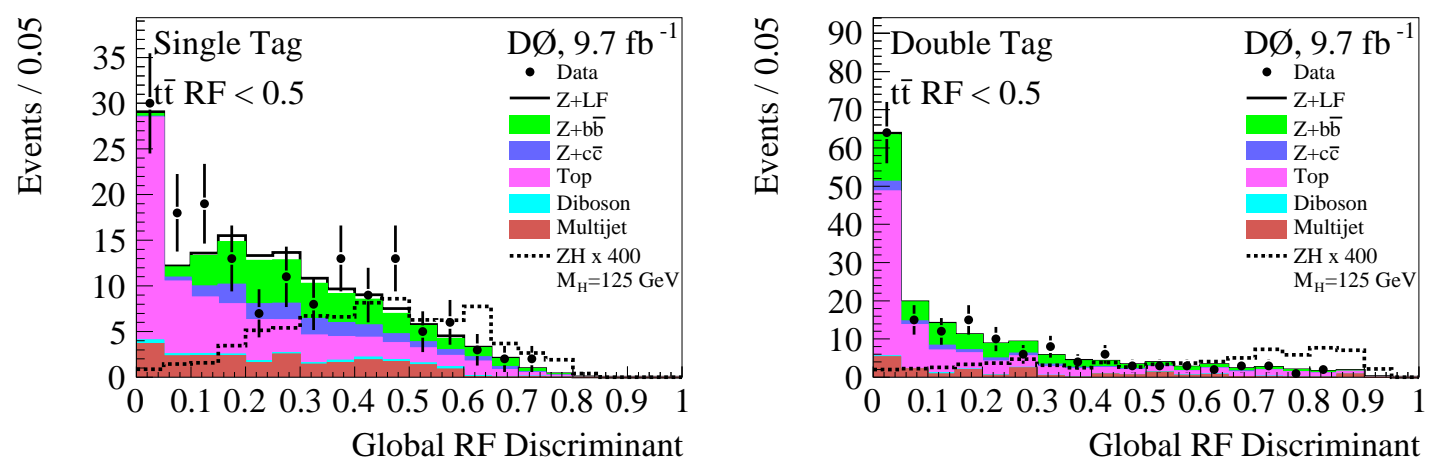

Figure 7.20: Distributions of Global RF discriminants for ST and DT events in the $t \bar{t}$ depleted region for $M_{H}=125 \mathrm{GeV}$. The signal distributions correspond to the $M_{H}$ used for the RF training and are scaled by a factor of 400 . 
8

\section{Systematic Uncertainties}

The distributions of final MVA discriminants for the observation in data and for the signal and background processes are used as inputs for statistical calculations to extract limits on the SM Higgs boson production cross section and branching fraction. The accuracy of these calculations, as well as the signal sensitivity of our searches, is determined by the impact of both statistical and systematic uncertainties on the normalization and shape of the final discriminant distributions.

The systematic uncertainties arising from various sources, such as reconstruction of objects, calibration of measured quantities and $\mathrm{MC}$ generations, are propagated to the final MVA discriminant by individually varying each source of systematics by $\pm \sigma$ (where $\sigma$ is the size of the relevant uncertainty at one standard deviation) and re-performing a full analysis or just re-evaluating the performance of the trained RF classifier on the modified testing sample. For the most significant uncertainties resulting from the calibration of jet energies, jet resolution, jet reconstruction efficiency and deriving the template for multijet background from data, we need to vary each of corresponding source of uncertainties and reperform the entire analysis starting from the event selection. The systematic uncertainties are classified into two categories:

- Flat systematic uncertainties: affect only the normalization of a signal or background process, and thereby result in varying all bins of the corresponding RF distributions by a constant factor.

- Shape systematic uncertainties: affect each bin of the final discriminant distribution differently, causing a difference in shape between the nominal distribution and the distribution with uncertainty propagated.

We assess the impact of systematic uncertainties based on one standard deviation variations on signals and backgrounds for each of the jet multiplicity and $b$-tag channels. A description of the propagation of systematic uncertainties and their status as shape or flatonly for each of $H \rightarrow W W \rightarrow \mu \nu j j$ and $Z H \rightarrow \ell \ell b b$ analyses is given in the following sections. 


\subsection{Systematic Uncertainties in $H \rightarrow W W \rightarrow \mu \nu j j$ Analysis}

\subsubsection{Flat Systematics Uncertainties}

- The theoretical uncertainties applied to the production cross sections times branching ratios of signal and background processes are $7 \%$ for $t \bar{t}$ and single top quark production $([85,86]), 6 \%$ for diboson $(W W, W Z$ and $Z Z$ ) production ( $[87]), 6 \%$ for $V+$ lp production, $20 \%$ for the $V+$ hf production (estimated from MCFM [84,75]), $6 \%$ for the $H \rightarrow W W \rightarrow \mu \nu j j$ production and approximately $3 \%$ for $W H \rightarrow \ell \nu b b$ and $W H \rightarrow$ $W W W \rightarrow \ell \nu j j j j$ production at the hypothesized mass of $M_{H}=125 \mathrm{GeV}$.

- The uncertainty in the measurement of luminosity affects the expected rates of all signal and background processes. We include a flat uncertainty of $6 \%$ on the total integrated luminosity [107].

- The parton distribution function (PDF) uncertainty is estimated by calculating the change in event yield for each of the twenty PDF eigenvectors in the CTEQ6M model used for MC generation. These twenty uncertainties are then added in quadrature to produce a single flat uncertainty. A flat PDF uncertainty of $2 \%$ is included in our analyses.

- For the searches in the low mass regions $\left(100 \leq M_{H} \leq 150 \mathrm{GeV}\right)$, we assigned flat systematic uncertainties of $3 \%$ and $5 \%$ affecting all signal and background events in zero and one-loose $b$-tag channels to account for the uncertainties in the taggability and $b$-jet tagging efficiency, respectively.

- The normalization of the multijet background is derived from the simultaneous fit of the multijet template and the $V+$ jets $M C$ sample to the data. We apply a flat uncertainty on the multijet rate of $20 \%$ to account for the uncertainty in the multijet normalization scale factor. Since the $V+$ jets experimental scaling factors for the threejet channel is different from unity, we also apply an additional systematic uncertainty on the $V+$ jets samples in this channel. The size of this uncertainty is taken as the uncertainty of $V+$ jets K-factor obtained from the fit described in Sec. 5.5.

- We include an uncertainty of $3 \%$ on the efficiency of muon identification and an uncertainty of $2 \%$ on the efficiency of jet identification.

\subsubsection{Shape Systematics Uncertainties}

- We assess systematic changes in the shape of the RF discriminant distribution due to uncertainty in the jet energy scale (JES) by shifting the JES correction for MC jet energy up and down by one standard deviation and repeating the full analysis with the resulting jets instead of the nominal jets. In addition to the nominal sample, we produce JES systematic samples for each of jet multiplicity and $b$-tag channels. The 
$\mathrm{RF}$ trained on the nominal sample is then applied to these systematic samples. The resulting final discriminant distributions are different from the nominal distributions and used in the statistical analysis to quantify the systematic uncertainty.

- The uncertainty on jet energy resolution (JSSR) is propagated to the RF discriminant distribution through similar steps as for the JES uncertainty. Jets are reconstructed using JSSR parameters fluctuated at $\pm 1 \sigma$ to produce two independent JSSR systematic samples for each channel. The RF trained on the nominal sample is then applied to JSSR systematic samples to obtain corresponding RF discriminant distributions which are different in shape from the nominal RF distributions.

- We assess systematic changes in the shape of the RF distribution due to uncertainty in jet identification efficiency (JetID) by shifting the removal scale factor down by $1 \sigma$, repeating the selection process, and applying the trained RF on the JetID systematic sample. For the $+1 \sigma$ JetID variation, we symmetrize the distribution of the final discriminant corresponding to the $-1 \sigma$ JetID variation.

- The jet vertex confirmed (VC) scale factor is implemented through the use of event weights. Therefore, the impact of the uncertainty on the VC scale factor to the RF discriminants is evaluated through event weights by shifting the VC scale factor up or down by one standard deviation.

- Three shape dependent systematics are considered for the ALPGEN and PYTHIA modeling of $V+$ jets production. To account for uncertainties from ALPGEN reweighting, we include separate uncertainties on each of the five fitted functions used to apply the reweighting. The adjusted functions are calculated by shifting the parameter responsible for the largest shape variation of the fit by $\pm 1 \sigma$, and then calculating the remaining parameters for the function using the covariance matrix obtained from the functional fit. We apply an uncertainty on the MLM matching ALPGEN tree-level matrix element generators with PYTHIA parton showers to $V+$ jets events. Additionally, we include an uncertainty for the underlying event (AlpUE) modeled in ALPGEN and PYTHIA [108]. Both the MLM and AlpUE uncertainties are varied independently and applied event-by-event.

- The uncertainty on the shape of our multijet sample driven from data is determined by relaxing the triangular cut on the transverse mass $M_{T}^{W}$ of the leptonic $W$ for vetoing multijet background (Sec. 6.1.2) to $M_{T}^{W}>30 \mathrm{GeV}-0.5 \times \mathbb{E}_{T}$ and repeating the analysis with this selection in place. The positive and negative variations are taken to be symmetric.

A summary of the magnitude of the systematic uncertainties and their effect on shape or normalization in RF discriminant distribution is given in Table 8.1 
Table 8.1: Systematic uncertainties for the $H \rightarrow W W \rightarrow \mu \nu j j$ analysis. Flat systematic uncertainties are given as a relative change in the rate in percent. The uncertainty on the experimental K-factor resulting in the uncertainty in normalization of the $V+$ jets $\mathrm{MC}$ sample is only applied to events with three jets. Systematic uncertainties for signals shown in this table are obtained for $M_{H}=125 \mathrm{GeV}$.

\begin{tabular}{||l|c|c|c|c|c|c||}
\hline \hline Contribution & Signals & $V+\mathrm{lp}$ & $V+\mathrm{hf}$ & Top & Dibosons & Multijets \\
\hline Theoretical X-Section & $3-6 \%$ & $6 \%$ & $20 \%$ & $7 \%$ & $6 \%$ & - \\
Luminosity & $6 \%$ & $6 \%$ & $6 \%$ & $6 \%$ & $6 \%$ & - \\
PDFs & $2 \%$ & $2 \%$ & $2 \%$ & $2 \%$ & $2 \%$ & - \\
Efficiency of $\mu$ ID & $3 \%$ & $3 \%$ & $3 \%$ & $3 \%$ & $3 \%$ & - \\
Efficiency of Jet ID & $2 \%$ & $2 \%$ & $2 \%$ & $2 \%$ & $2 \%$ & - \\
Normalization & - & $5 \%(3$ jets $)$ & $5 \%(3$ jets $)$ & - & - & $20 \%$ \\
Jet Taggability & $3 \%$ & $3 \%$ & $3 \%$ & $3 \%$ & $3 \%$ & $3 \%$ \\
$b$-tagging Efficiency & $5 \%$ & $5 \%$ & $5 \%$ & $5 \%$ & $5 \%$ & - \\
Jet Energy Scale & Shape & Shape & Shape & Shape & Shape & - \\
Jet Energy Resolution & Shape & Shape & Shape & Shape & Shape & - \\
Jet Identification & Shape & Shape & Shape & Shape & Shape & - \\
VC Scale Factor & Shape & Shape & Shape & Shape & Shape & - \\
Alpgen MLM & - & Shape & Shape & - & - & - \\
AlgUE & - & Shape & Shape & - & - & - \\
Multijet Shape & - & - & - & - & & Shape \\
\hline \hline
\end{tabular}

\subsection{Systematic Uncertainties in $Z H \rightarrow \ell \ell b b$ Analysis}

Similar to the $H \rightarrow W W \rightarrow \mu \nu j j$ analysis, we assess the impact of systematic uncertainties on both the normalization and the shape of the predicted global RF distribution for each of the signal and background processes in the $Z H \rightarrow \ell \ell b b$ analysis $[11,106]$. We include uncertainties associated with the theoretical SM cross section for the $Z H$ signal process $(6 \%)$, the $t \bar{t}(10 \%)$, diboson (7\%), the $Z+\mathrm{lp}(6 \%)$ and the $Z+\mathrm{hf}(20 \%)$ background processes. The uncertainties on the integrated luminosity and the lepton identification efficiencies are absorbed by the uncertainties on the normalization applied to each of signal and background processes. Other sources of flat systematic uncertainties include the uncertainty on the normalization of the multijet background, which is determined from uncertainties on the normalization fit (typically around 10\%), and the PDF uncertainty.

Sources of systematic uncertainty affecting the shapes of the final RF discriminant distributions are the jet energy scale, jet energy resolution, jet identification efficiency, and $b$-tagging efficiency. These shape uncertainties are assessed by repeating the full analysis with each source of uncertainty varied up and down by one standard deviation. We also include uncertainties in modeling of the underlying event and the MLM matching process, which are only applied to ALPGEN $Z+$ jets events. A summary of the magnitude of systematic uncertainties and their effect on shape or normalization in the final RF discriminant distribution is given in Table 8.2. 
Table 8.2: Systematic uncertainties for the $Z H \rightarrow \ell \ell b b$ analysis. Flat systematic uncertainties are given as the relative change in the rate in percent. Systematic uncertainties for the $Z H$ signal shown in this table are obtained for $M_{H}=125 \mathrm{GeV}$.

\begin{tabular}{||l|c|c|c|c|c|c|c||}
\hline \hline Contribution & $Z H$ & $Z+\mathrm{lp}$ & $Z+b \bar{b}$ & $Z+c \bar{c}$ & Dibosons & $t \bar{t}$ & Multijet \\
Theoretical X-Section & $6 \%$ & $6 \%$ & $20 \%$ & $20 \%$ & $7 \%$ & $10 \%$ & - \\
PDFs & $0.6 \%$ & $1.0 \%$ & $2.4 \%$ & $1.1 \%$ & $0.7 \%$ & $5.9 \%$ & - \\
Normalization & $5.1 \%$ & $5.1 \%$ & $5.1 \%$ & $5.1 \%$ & $5.1 \%$ & $5.1 \%$ & $10 \%$ \\
Jet Taggability & Shape & Shape & Shape & Shape & Shape & Shape & - \\
$b$-tagging Efficiency & Shape & Shape & Shape & Shape & Shape & Shape & - \\
Jet Energy Scale & Shape & Shape & Shape & Shape & Shape & Shape & - \\
Jet Energy Resolution & Shape & Shape & Shape & Shape & Shape & Shape & - \\
Jet Identification & Shape & Shape & Shape & Shape & Shape & Shape & - \\
Alpgen MLM & - & Shape & Shape & Shape & - & - & - \\
AlgUE & - & Shape & Shape & Shape & - & - & - \\
Multijet Shape & - & - & - & - & - & & Shape \\
\hline \hline
\end{tabular}


9

\section{Results of the Higgs Boson Searches}

The MVA discriminant distributions, together with their associated uncertainties, are used as inputs to a procedure of statistical calculations to derive upper limits on the Higgs boson production cross section multiplied by the corresponding branching fraction $\left(\sigma_{\mathrm{H}}\right)$. Our limits setting procedure is based on the modified frequentist $\mathrm{CL}_{s}$ approach $[109,110,111]$ and is repeated for each hypothesized mass of the Higgs boson. In this chapter, the $\mathrm{CL}_{\mathrm{S}}$ method of limit setting is described first, and then the results of the SM Higgs boson searches in the $\ell \nu j j$ and $Z H \rightarrow \ell \ell b b$ analyses are presented, along with the interpretation of our Higgs boson searches in models containing a fourth generation of fermions and a model with a fermiophobic Higgs boson for the $\ell \nu j j$ final state. Our search results in the $\ell \nu j j$ and $Z H \rightarrow \ell \ell b b$ analyses are included in the results of the $\mathrm{D} \varnothing$ and Tevatron combinations, which are described in the last section of this chapter.

\section{1 $\mathrm{CL}_{\mathrm{S}}$ Method for Limit Calculation}

\subsubsection{Procedure for Limit-Setting}

The limit calculations in our analyses are performed with the modified frequentist technique $\mathrm{CL}_{\mathrm{s}}[109,110]$ implemented in the Confidence Level Limit Evaluator (COLLIE) package [112]. We first consider the following two hypotheses:

- The background-only hypothesis (B), in which only background contributions are present.

- The signal-plus-background hypothesis $(\mathrm{S}+\mathrm{B})$, in which both signal and background contributions are present.

We assume that the data collected by an experiment is stochastically sampled from a Poisson parent distribution. Thus, a set of pseudo-data for each of background-only and the signal- 
plus-background hypotheses is simulated via sampling randomly from a Poisson distribution with a mean value given by the expected number of events for the hypothesis under the investigation. The probabilities that the data (either observed data or pseudo-data) are drawn from distributions predicted under background-only and the signal-plus-background hypotheses are given as:

$$
\begin{aligned}
P(\text { data } \mid \mathrm{B}) & =\quad \frac{b^{d} \cdot e^{-b}}{d !}, \\
P(\text { data } \mid \mathrm{S}+\mathrm{B}) & =\frac{(s+b)^{d} \cdot e^{-(s+b)}}{d !},
\end{aligned}
$$

where $b$ and $s$ correspond, respectively, to the number of predicted background and signal events, and $d$ corresponds to the number of events in either observed data or pseudo-data. The likelihood ratio is then constructed from $P($ data $\mid \mathrm{B})$ and $P($ data $\mid \mathrm{S}+\mathrm{B})$ probabilities, and taking its logarithm (LLR), we define:

$$
\Gamma \equiv-2 \ln (Q)=-2 \ln \left(\frac{P(\text { data } \mid \mathrm{S}+\mathrm{B})}{P(\text { data } \mid \mathrm{B})}\right)
$$

which is used as a test statistic in the $\mathrm{CL}_{\mathrm{S}}$ method to quantify the degree of compatibility of the data with the background-only and signal-plus-background hypotheses. The probability distribution function of the test statistic $(\Gamma)$ for each hypothesis is numerically constructed via generating a set of pseudo-data for the corresponding hypothesis by running artificial pseudo-experiments based on Poisson statistics.

Since the inputs to COLLIE are MVA discriminant distributions, which are histograms including a number of bins and provided from separate channels, the join likelihood ratio is formed to combine the likelihood ratios of Poisson distributions from each individual bin and channel:

$$
\begin{aligned}
Q^{\prime} & =\prod_{i=1}^{N_{\mathrm{C}}} \prod_{j=1}^{N_{\text {bins }}} \frac{e^{-\left(s_{i j}+b_{i j}\right)} \cdot\left(s_{i j}+b_{i j}\right)^{d_{i j}} / d_{i j} !}{e^{-\left(b_{i j}\right)} \cdot\left(b_{i j}\right)^{d_{i j}} / d_{i j} !} \\
Q^{\prime} & =\prod_{i=1}^{N_{\mathrm{C}}} \prod_{j=1}^{N_{\text {bins }}} e^{-s_{i j}}\left(\frac{s_{i j}+b_{i j}}{b_{i j}}\right)^{d_{i j}},
\end{aligned}
$$

where the index $i$ runs over the number of channels and the index $j$ runs over the number of bins in each channel. Consequently, the combined LLR for separate channels and bins is obtained by summing LLR values over all bins and channels:

$$
\Gamma \equiv-2 \ln \left(Q^{\prime}\right)=2 \sum_{i}^{N_{\mathrm{C}}} \sum_{j}^{N_{\text {bins }}}\left[s_{i j}-d_{i j} \cdot \ln \left(1+\frac{s_{i j}}{b_{i j}}\right)\right] .
$$

We obtain the observed LLR from Eqn. (9.2) by taking $d_{i j}$ as the observed data for the bin $j$ of the MVA discriminant distribution for observed data in the $i$ channel. To construct 
the LLR for each of the background-only and signal-plus-background hypotheses, we first generate an individual ensemble of pseudo-data for each hypothesis. The number of pseudodata events in each bin is determined by Poisson trials per bin, which are seeded with the mean value taken from the sum of contributing processes in that bin for the relevant hypothesis $\left(s_{i j}+b_{i j}\right.$ for the $\mathrm{S}+\mathrm{B}$ hypothesis, or $b_{i j}$ for the B hypothesis). The probability distribution function of the LLR for each of the background-only and signal-plus-background hypotheses is populated via evaluating Eqn. (9.2) on the corresponding ensemble of pseudodata. Examples of the observed LLR and two distributions of LLR corresponding to the background-only hypothesis (B Only LLR) and signal-plus-background hypothesis ( $\mathrm{S}+\mathrm{B}$ LLR) are shown in Fig. 9.1.

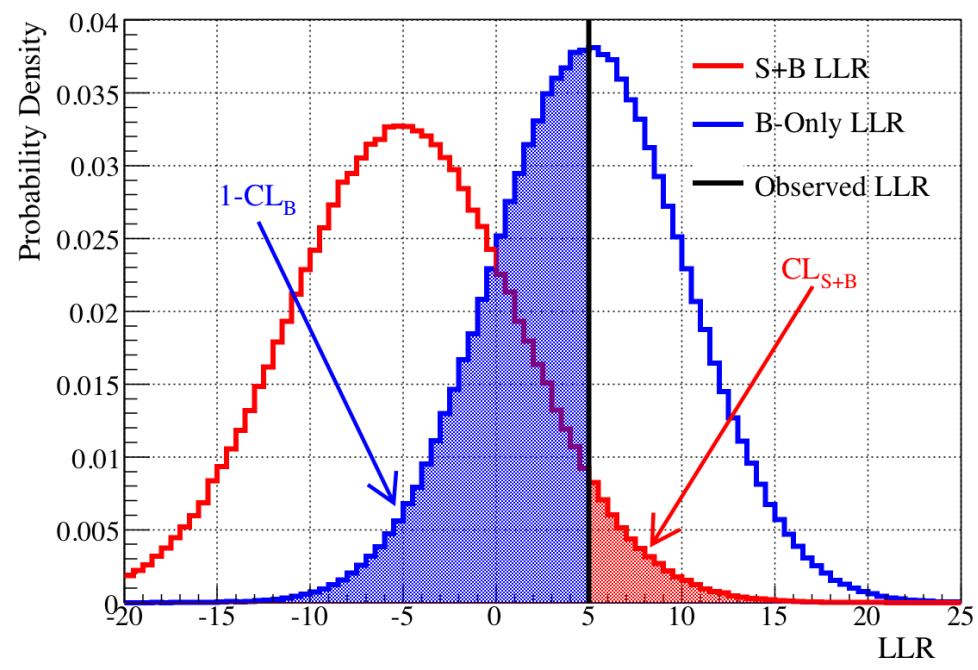

Figure 9.1: Examples of LLR evaluated for observed data (black) and distributions of LLR evaluated for backround-only (blue) and signal-plus-background (red) hypotheses. The shaded red (blue) correspond to the values $\mathrm{CL}_{\mathrm{S}+\mathrm{B}}\left(1-\mathrm{CL}_{\mathrm{B}}\right)$.

Upon inspection of Eqn. (9.2), the median LLR value is positive for the background-only hypothesis $\left(d_{i j}=b_{i j}\right)$ and is negative for the signal-plus-background hypothesis $\left(d_{i j}=\right.$ $s_{i j}+b_{i j}$ ). If the signal rate increases, the distribution of LLR for signal-plus-background hypothesis in Fig. 9.1 will move further to the left while the distribution of LLR for background-only hypothesis will move further to the right. Having the LLR distributions for the two hypotheses constructed, we define the confidence level for each hypothesis as following

- The confidence level for the signal-plus-backround hypothesis, $\mathrm{CL}_{\mathrm{S}+\mathrm{B}}$, is the probability for this hypothesis to produce a LLR more background-like than a reference LLR (for example, observed LLR or the median LLR for the backround-only hypothethis) denoted as $\Gamma_{0}$. It is evaluated by integrating the LLR distribution for signal-plus-background hypothesis from the value of $\Gamma_{0}$ to infinity. In Fig. 9.1, the shaded red corresponds to the value of $\mathrm{CL}_{\mathrm{S}+\mathrm{B}}$. The $\mathrm{CL}_{\mathrm{S}+\mathrm{B}}$ is also the $p$-value for the 
signal-plus-background hypothesis.

- The confident level for the backround-only hypothesis, $\mathrm{CL}_{\mathrm{B}}$, is the probability for this hypotheses to produce LLR more background-like than the reference LLR. It is evaluated by integrating the corresponding LLR distribution from $\Gamma_{0}$ to infinity. The value of $\left(1-\mathrm{CL}_{\mathrm{B}}\right)$ presents the $p$-value for the background-only model. In Fig. 9.1, the shaded blue corresponds to the $p$-value for the background-only hypothesis.

To compute the upper limits on the signal cross section to exclude the signal-plus-background hypothesis at a certain confidence level, we evaluate the ratio of the confidence levels for the two hypotheses:

$$
\mathrm{CL}_{\mathrm{S}}=\frac{\mathrm{CL}_{\mathrm{S}+\mathrm{B}}}{\mathrm{CL}_{\mathrm{B}}}
$$

As the signal rate increases the $\mathrm{CL}_{\mathrm{S}+\mathrm{B}}$ will decrease while $\mathrm{CL}_{\mathrm{B}}$ will increase, therefore, the $\mathrm{CL}_{\mathrm{S}}$ in Eqn. (9.3) will decrease. In order to use the $\mathrm{CL}_{\mathrm{S}}$ as the modified-Frequentist statistic to quote a $95 \%$ confident level limit, the signal rate is iteratively changed by scaling

the signal cross section in steps until it reaches the desired upper limit $\sigma_{\text {sig }}^{95 \%}$ where the $\mathrm{CL}_{\mathrm{S}}$ satisfies:

$$
1-\mathrm{CL}_{\mathrm{S}}>0.95 \text { or } \mathrm{CL}_{\mathrm{S}}<0.05
$$

For the SM Higgs boson searches, we present the upper limits on the Higgs boson production cross section in units of the $\mathrm{SM}$ prediction $\left(\sigma_{\text {sig }}^{\mathrm{SM}}\right)$ :

$$
R_{95}=\sigma_{\text {sig }}^{95} / \sigma_{\text {sig }}^{\mathrm{SM}}
$$

The observed upper limit, $R_{95}^{\text {obs }}$, is obtained from Eqn. (9.5) by taking the value $\Gamma_{0}$ of the reference LLR, which is used to calculated confident levels $\mathrm{CL}_{\mathrm{S}+\mathrm{B}}$ and $\mathrm{CL}_{\mathrm{B}}$, as the observed LLR. The expected upper limit, $R_{95}^{\text {exp }}$, is computed similarly by using the median LLR value expected in the background-only hypothesis as the reference LLR. The procedure of limit-setting needs to be repeated for each each assumed value of $M_{H}$.

\subsubsection{Treatment of Uncertainties}

The predicted number of signal and background events in each bin of the MVA discriminant distributions, which are used as the mean values of the Poisson statistics for generating pseudo-data, are determined with uncertainties as described in Sec. 8.1. In order to incorporate effects of these uncertainties in the limit-setting procedure, we fluctuate the signal and background predictions $\left(s_{i j}\right.$ and $\left.b_{i j}\right)$ around their nominal values and within their uncertainties when generating the pseudoexperiments used to compute $\mathrm{CL}_{\mathrm{S}+\mathrm{B}}$ and $\mathrm{CL}_{\mathrm{B}}$.

We include a nuisance parameter for each independent source of systematic uncertainty and each parameter may affect the predictions of several sources of signal and background in 
different channels, thus accounting for correlations. Gaussian prior densities are assumed for the nuisance parameters, and systematic uncertainties are parametrized by the dependence of signal and background predictions on the whole set of nuisance parameters $(\vec{\theta})$. The parameters present the uncertainties on the signal and background predictions caused by the corresponding systematics source. The nominal value $p_{i}^{0}$ of the predicted number of events in bin $i$ for the background-only or signal-plus-background hypothesis is defined by the central values of the set of nuisance parameters:

$$
p_{i}^{0}\left(\overrightarrow{\theta_{k}}\right)=\sum_{j}^{N_{\text {proc }}} p_{i j}\left(\theta_{1}^{0}, \ldots, \theta_{k}^{0}, \ldots\right),
$$

where the index $j$ run over the number of contributing processes for the relevant hypothesis $N_{\text {proc. }}$.

For each pseudoexperiment, the value of each nuisance parameter $\theta_{k}$ is randomly drawn from its Gaussian distribution with the width $\sigma_{k}$. For a given set of fluctuated parameters, the predicted mean values used as the Poisson mean for generating pseudo-data are rederived as [112]:

$$
\begin{aligned}
p_{i}\left(\overrightarrow{\theta_{k}}\right) & =\sum_{j}^{N_{\text {proc }}} p_{i j}\left(\theta_{1}, \ldots, \theta_{k}, \ldots\right), \\
p_{i}\left(\overrightarrow{\theta_{k}}\right) & =\sum_{j}^{N_{\text {proc }}} p_{i j}^{0} \prod_{k=1}^{N_{\text {par }}}\left(1+R_{k} \sigma_{i j k}\right),
\end{aligned}
$$

where the index $k$ runs over the number of nuisance parameters in the model, $\sigma_{i j k}=$ $\sigma_{k} \cdot \frac{\partial p_{i j}\left(\overrightarrow{\theta_{k}}\right) / \delta \theta_{k}}{p_{i j}\left(\theta_{k}^{0}\right)}$ defines the fractional change in the number of events for the specified nuisance parameter $k$ for the specified event source $\mathrm{j}$, and $R_{k}=\left(\theta_{k}-\theta_{k}^{0}\right) / \sigma_{k}$ represents the deviation from the central value of the nuisance parameter in units of the Gaussian width $\sigma_{k}$.

Each common systematics uncertainty, such as the uncertainties on predicted SM cross sections, identification efficiencies, energy calibration and the uncertainty on a measurement of the luminosity (Sec. 8.1), is taken to be correlated for each signal or background process across all channels. These correlations are considered in the process of simulating pseudoexperiments to ensure the pseudo-data are generated properly. As the nuisance parameter corresponding to each correlated uncertainty is sampled from a Gaussian distribution and used to calculate the mean value for each Poisson trial (Eqn.( 9.7)), it is randomly sampled only once per iteration. In this way, each correlated uncertainty is ensured to have the same fluctuation for all events of correlated processes and channels.

The statistical uncertainty associated with the number of Monte Carlo events per histogram bin of the inputs is also accounted in the calculation of the number of predicted signal and background events. This is done by performing an uncorrelated Gaussian fluc- 
tuation of the expectation for the number of events in each bin of each event source. The width of the Gaussian prior is set by the per-bin statistical uncertainty.

The inclusion of systematic uncertainties in the simulation of pseudoexperiments has the effect of broadening the LLR distributions and, thus, reducing the ability to resolve signallike excesses. In order to minimize the degrading effects of systematic uncertainties on the search sensitivity, we fit the predicted MVA discriminant templates corresponding to each signal-plus-background and background-only hypothesis to the pseudo-data for each hypothesis while letting the predicted event contribution from each process to vary within the systematic uncertainties. The likelihood used in the fits is the joint Poisson probability over the number of bins, and is parameterized as a function of the nuisance parameters and their uncertainties [111]. Maximization of the likelihood function is performed over the nuisance parameters and is repeated for each Poisson MC trial. The negative log-likelihood LLR in Eqn. (9.1) is re-defined to be the negative logarithm of likelihood maximized independently for the signal-plus-background and background-only hypotheses:

$$
\Gamma \equiv-2 \ln \left(Q\left(\text { data } \mid \vec{\theta}_{0}, \vec{\theta}_{1}\right)\right)=-2 \ln \left(\frac{P\left(\text { data } \mid \mathrm{S}+\mathrm{B}, \hat{\theta}_{1}\right)}{P\left(\text { data } \mid \mathrm{B}, \hat{\theta}_{0}\right)}\right)
$$

where $\vec{\theta}_{1}$ represents the set of nuisance parameters for the signal-plus-background hypoth-

esis, $\vec{\theta}_{0}$ represents the set of nuisance parameters for the background-only hypothesis, $\hat{\theta}_{1}$ represents the set of nuisance parameter values that maximize the likelihood for the signalplus-background hypothesis, and $\hat{\theta}_{0}$ represents the set of nuisance parameter values that maximize the likelihood for the background-only hypothesis.

Figure 9.2 shows the signal expectation at $M_{H}=125 \mathrm{GeV}$ and the observed data with the background subtracted as functions of the signal purity $s / b$ of the collected bins of the RF discriminant distributions, where $s$ is the number of signal and $b$ the number of background events in each bin, for the $H \rightarrow W W \rightarrow \mu \nu j j$ channel. The background prediction in the background-only hypothesis is fit to the corresponding pesudo-data, allowing the nuisance parameters to vary within their constraints. The signal expectation is shown with the SM cross section scaled by a factor of 10 and the uncertainties in the background predictions after the constrained fit are shown by the solid lines. A good agreement between data and predictions within the systematic uncertainties is indicated in Fig. 9.2.

\subsection{Results of the Higgs Boson Searches in $\ell \nu j j$ Analysis}

\subsubsection{Upper Limits on the SM Higgs Boson Production}

The RF discriminant distributions of the $H \rightarrow W W \rightarrow \mu \nu j j$ channel (Sec. 7.1) along with their associated systematic uncertainties are used to extract the upper limits on the SM Higgs boson production cross section multiplied by the corresponding branching fraction in 


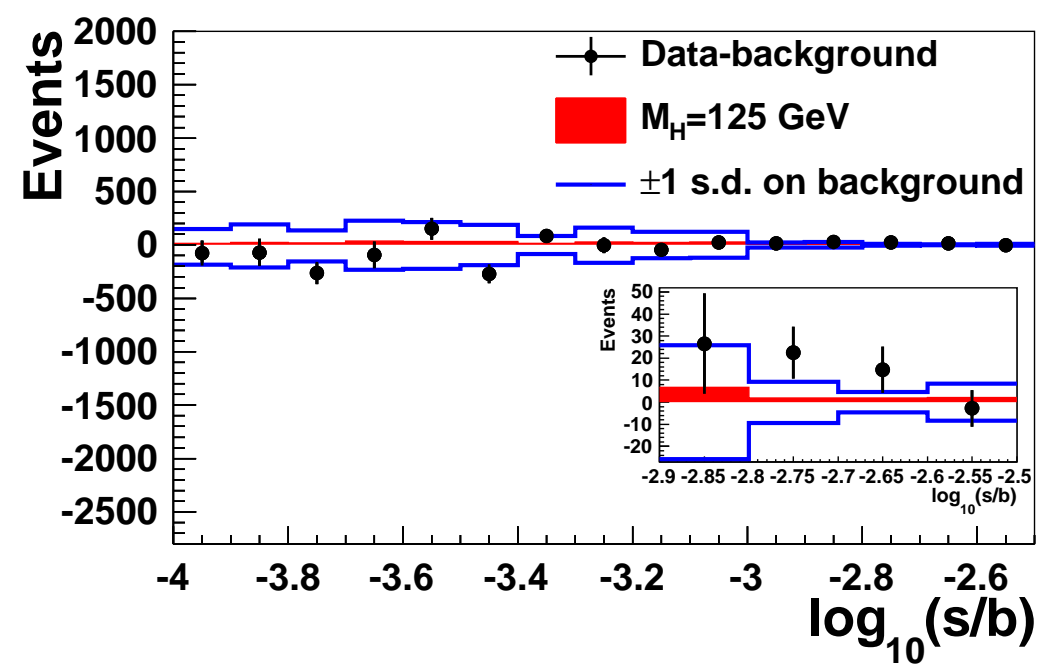

Figure 9.2: The MVA discriminant distributions for the expected signal at $M_{H}=125 \mathrm{GeV}$ and background-subtracted data rebinned as a function of $\log (s / b)$ for events in the $H \rightarrow W W \rightarrow \mu \nu j j$ channel, after the maximum likelihood fit to the observed data in the background-only hypothesis. The error bars on data points represent the statistical uncertainty only. The post-fit systematic uncertainties are represented by the solid lines. The signal distribution is shown with the SM cross section scaled by a factor of 10 . The inset gives an expanded view of the $\operatorname{high} \log (s / b)$ region.

units of the SM prediction for this channel. The upper limits are calculated separately at 21 discrete values of the Higgs boson mass, spanning the range 100-200 GeV and spaced in increments of $5 \mathrm{GeV}$. For the calculations in the low mass region, $100 \leq M_{H} \leq 150 \mathrm{GeV}$, we use the RF discriminant distributions of the four sub-samples from the zero $b$-tag and one-loose $b$-tag categories with two different jet multiplicities (two jets and three jets). In the high mass region, $155 \leq M_{H} \leq 200 \mathrm{GeV}$, two-jet and three-jet events are used for the limit calculations without $b$-tag requirements. To preserve the stability of the limit derivation procedure in regions of small background statistics, the binning of the RF discriminant distribution is chosen such that the relative statistical uncertainty on the signals plus background prediction is less than $15 \%$.

Figure 9.3 displays the LLR distributions for the $H \rightarrow W W \rightarrow \mu \nu j j$ analysis as a function of the hypothesized Higgs boson mas, $M_{H}$. Included in this figure are the median LLR values expected for the signal-plus-background hypothesis ( $\mathrm{LLR}_{\mathrm{S}+\mathrm{B}}$ ), backgroundonly hypothesis $\left(\mathrm{LLR}_{\mathrm{B}}\right)$, and the results observed in data $\left(\mathrm{LLR}_{\mathrm{OBS}}\right)$. The corresponding \pm 1 standard deviation (s.d.) and \pm 2 s.d. values for the $L_{L R}$ at each mass point are represented by the green and yellow shaded bands.

The separation between the $L_{L} R_{S+B}$ and $L L R_{B}$ distributions provides a measure of the discriminating power of the search and illustrates the effectiveness of the analysis to separate the the signal-plus-background and background-only hypotheses. The widths of the one- 


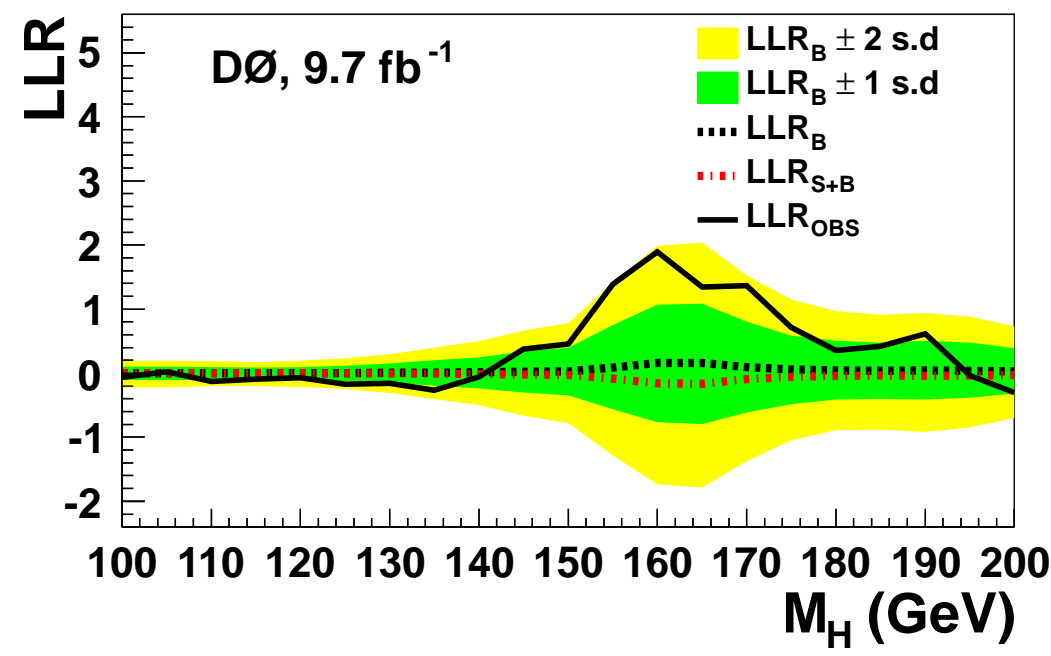

Figure 9.3: The expected and observed log-likelihood ratios (LLR) as functions of the hypothesized Higgs boson mass $M_{H}$ for the $H \rightarrow W W \rightarrow \mu \nu j j$ analysis. The solid line corresponds to the LLR obtained from the data. The dashed red and black lines correspond to the median LLR of the signal-plus-background and background-only hypotheses, respectively. The green and yellow shaded regions are the \pm 1 s.d. and \pm 2 s.d. values for the $\mathrm{LLR}_{\mathrm{B}}$ corresponding to the background-only hypothesis.

and two-s.d. $\mathrm{LLR}_{\mathrm{B}}$ bands indicate the fluctuations of the $\mathrm{LLR}_{\mathrm{B}}$ due to statistical fluctuations and effects of systematic uncertainties, assuming no signal. The value of LLR $\mathrm{OBS}_{\mathrm{B}}$ relative to the $L_{L L R}+B$ and $L_{L R}$ indicates whether the data distribution more closely resembles the distributions expected if a signal is present or only background is present. The significance of departures of $L L R_{\mathrm{OBS}}$ from $\mathrm{LLR}_{\mathrm{B}}$ can be evaluated by the width of the $\mathrm{LLR}_{\mathrm{B}}$ uncertainty bands. Figure 9.3 shows that the observed data is consistent with the background-only hypothesis for $145<M_{H}<195 \mathrm{GeV}$ and more compatible with the signal-plus-background hypothesis for $120<M_{H}<145 \mathrm{GeV}$.

We calculate the upper limit on the SM Higgs production at 95\% C.L. as a function of the Higgs boson mass $M_{H}$, and present the result in units of the predicted SM rate, $\sigma_{\mathrm{LM}} / \sigma_{S M}$, where $\sigma_{\mathrm{LM}}$ and $\sigma_{\mathrm{SM}}$ are the upper limit and the standard model prediction for the Higgs boson production cross section. The value of LLR ${ }_{\mathrm{OBS}}$ measured in data is taken as the LLR reference when calculating the $\mathrm{CL}_{\mathrm{S}+\mathrm{B}}$ and $\mathrm{CL}_{\mathrm{B}}$ confidence levels used to extract the observed limit, whereas the value of $\mathrm{LLR}_{\mathrm{B}}$ for the background-only hypothesis is taken as the LLR reference to set the expected limits. The expected and observed upper limits on $\sigma_{\mathrm{H}}$ at $95 \%$ C.L. extracted from $H \rightarrow W W \rightarrow \mu \nu j j$ analysis for each hypothesized Higgs boson mass in the region $100 \leq M_{H} \leq 200 \mathrm{GeV}$ are presented in Fig. 9.4 and Table 9.1. The $H \rightarrow W W \rightarrow \mu \nu j j$ analysis is most sensitive to the search for the SM Higgs boson at the hypothesized mass of $165 \mathrm{GeV}$, where we expect (observe) the upper limit of 6.14 (4.38) times the SM cross section. For the mass $M_{H}=125 \mathrm{GeV}$, we expect an upper limit at 
$43.6 \times \sigma_{\mathrm{SM}}$ and observe the upper limit at $83.8 \times \sigma_{\mathrm{SM}}$.

We combine individual searches from all sub-analyses $(H \rightarrow W W \rightarrow \ell \nu j j, W H \rightarrow \ell \nu j j$, and $W H \rightarrow W W W \rightarrow \ell \nu j j j j$ ) for different $b$-tag, jet multiplicity and lepton flavor categories in the $\ell \nu j j$ final state [10]. The distributions of the observed LLR and the median values of LLR for background-only and signal-plus-background hypotheses for each hypothesized mass $M_{H}$ are presented in Fig. 9.5. The expected and observed upper limits on $\sigma_{\mathrm{H}}$ at $95 \%$ C.L. relative to the $\mathrm{SM}$ rate for the mass region $90 \leq M_{H} \leq 200 \mathrm{GeV}$ are shown in Fig. 9.5 and summarized in Table 9.2. For the mass $M_{H}=125 \mathrm{GeV}$, the observed and expected upper limit are $5.8 \times \sigma_{\mathrm{SM}}$ and $4.7 \times \sigma_{\mathrm{SM}}$, respectively.

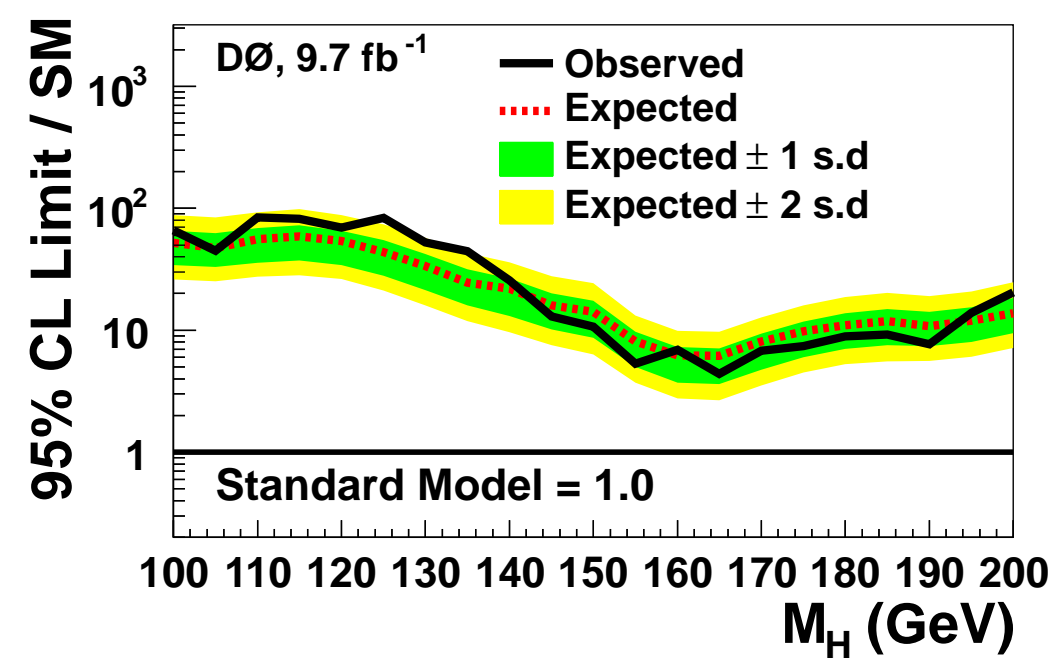

Figure 9.4: The expected and observed 95\% C.L. upper limits on SM Higgs boson production for the $H \rightarrow W W \rightarrow \mu \nu j j$ channel. The limits are presented as ratios to the expected SM prediction. The dashed line corresponds to the expected limit, and the solid line corresponds to the limit observed in data. The shaded regions are the \pm 1 s.d. and \pm 2 s.d. values for the expected limit.

\begin{tabular}{||c|ccccccccccc||}
\hline \hline & \multicolumn{10}{|c|}{ Upper limits $\left(\boldsymbol{\sigma}_{\mathrm{LM}} / \boldsymbol{\sigma}_{\mathrm{SM}}\right)$} & for $\boldsymbol{H} \boldsymbol{\boldsymbol { W }} \boldsymbol{W} \rightarrow \boldsymbol{\mu} \boldsymbol{\nu} \boldsymbol{j} \boldsymbol{j}$ channel \\
\hline$M_{H}(\mathrm{GeV})$ & 100 & 105 & 110 & 115 & 120 & 125 & 130 & 135 & 140 & 145 & 150 \\
\hline Expected & 52.1 & 47.1 & 55.9 & 58.7 & 53.7 & 43.6 & 33.5 & 24.5 & 21.8 & 16.0 & 14.2 \\
Observed & 66.1 & 44.7 & 83.8 & 82.3 & 69.5 & 83.8 & 52.9 & 44.5 & 25.6 & 13.0 & 10.7 \\
\hline \hline$M_{H}(\mathrm{GeV})$ & 155 & 160 & 165 & 170 & 175 & 180 & 185 & 190 & 195 & 200 & \\
\hline Expected & 8.11 & 6.23 & 6.14 & 8.06 & 9.82 & 10.96 & 11.9 & 10.8 & 12.0 & 13.8 & \\
Observed & 5.34 & 6.88 & 4.38 & 6.80 & 7.41 & 8.94 & 9.19 & 7.70 & 13.9 & 20.5 & \\
\hline \hline
\end{tabular}

Table 9.1: The expected and observed 95\% C.L. limits, as a function of the Higgs boson mass $M_{H}$, presented as ratios of production cross section times branching fraction to the expected SM prediction. 

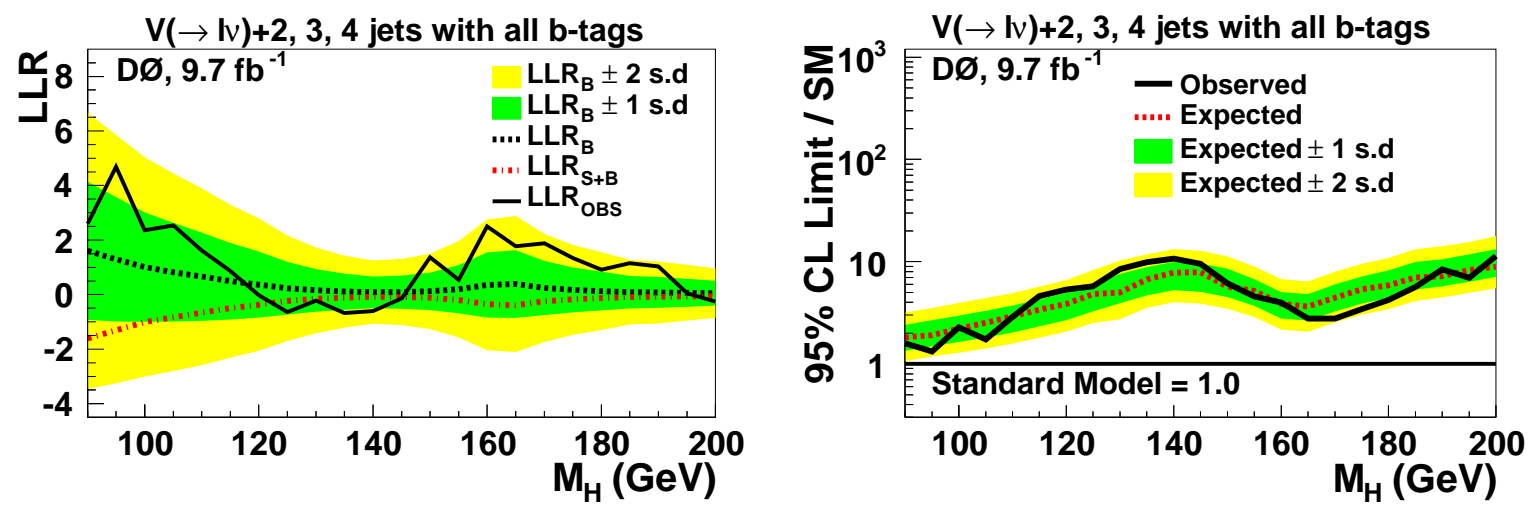

Figure 9.5: The LLR (left) and the 95\% C.L. upper limit (right) as functions of Higgs boson mass for the $\ell \nu j j$ analysis. The LLR plot shows the background-only expectation $\left(\mathrm{LLR}_{\mathrm{B}}\right)$, the signal-plus-background expectation $\left(\mathrm{LLR}_{\mathrm{S}+\mathrm{B}}\right)$, the observed data $\left(\mathrm{LLR}_{\mathrm{OBS}}\right)$. The green and yellow bands are the \pm 1 s.d. and \pm 2 s.d on the background-only expectation. The $95 \%$ C.L. limit on the Higgs boson production cross section is shown as a ratio of the SM cross section. The black dashed line shows the expected limit, the solid black line shows the observed limit [10].

\begin{tabular}{||c|cccccccccccc||}
\hline \hline \multicolumn{10}{c|}{ Upper limits $\left(\boldsymbol{\sigma}_{\mathbf{L M}} / \boldsymbol{\sigma}_{\mathbf{S M}}\right)$} & for $\boldsymbol{\ell} \boldsymbol{\nu} \boldsymbol{j} \boldsymbol{j}$ analysis \\
\hline$M_{H}(\mathrm{GeV})$ & 90 & 95 & 100 & 105 & 110 & 115 & 120 & 125 & 130 & 135 & 140 & 145 \\
\hline Expected & 1.8 & 1.9 & 2.2 & 2.5 & 2.9 & 3.4 & 3.8 & 4.7 & 5.0 & 6.7 & 7.8 & 7.9 \\
Observed & 1.6 & 1.3 & 2.3 & 1.7 & 2.9 & 4.6 & 5.3 & 5.8 & 8.5 & 9.9 & 10.7 & 9.6 \\
\hline \hline$M_{H}(\mathrm{GeV})$ & 150 & 155 & 160 & 165 & 170 & 175 & 180 & 185 & 190 & 195 & 200 & \\
\hline Expected & 5.7 & 5.2 & 3.8 & 3.7 & 4.4 & 5.4 & 5.9 & 7.0 & 7.2 & 8.3 & 8.9 & \\
Observed & 6.1 & 4.6 & 4.0 & 2.8 & 2.8 & 3.4 & 4.2 & 5.7 & 8.4 & 6.9 & 11.4 & \\
\hline \hline
\end{tabular}

Table 9.2: The expected and observed 95\% C.L. limits, as a function of the Higgs boson mass $M_{H}$, presented as ratios of production cross section times branching fraction to the expected SM prediction for $\ell \nu j j$ analysis.

\subsubsection{Interpretation in Fourth Generation and Fermiophobic Higgs Boson Models}

We consider two simple extensions to the SM that can be tested with Higgs boson search results at the Fermilab Tevatron Collider:

- The models with a sequential fourth generation of fermions (SM4), where the additional fourth-generation fermions have masses much larger than those of the three known generations [38, 39]

- The Fermiophobic Higgs boson model (FHM), where the Higgs boson has modified couplings [40].

The interpretations of our results in the SM4 and the Fermiophobic Higgs boson model are described below. 


\section{Fourth Generation Interpretation}

We consider two scenarios for the masses of the fourth-generation fermions, low-mass and high-mass. The masses of the fourth-generation neutrino and the fourth-generation charged lepton in the low-mass scenario are set at $m_{\nu 4}=80 \mathrm{GeV}$ and $m_{\ell 4}=100 \mathrm{GeV}$, in order to satisfy experimental constraints $[113,39]$ and to have maximum impact on the Higgs boson decay branching fractions. In the high-mass scenario, the masses of the fourth-generation neutrino and the fourth-generation charged lepton are set at $m_{\nu 4}=m_{\ell 4}=1 \mathrm{TeV} / c^{2}$, so that the fourth-generation leptons do not modify the decay branching fractions of the Higgs boson relative to the SM. In both scenarios, the mass of the fourth-generation down-type quark is set to $m_{d 4}=400 \mathrm{GeV}$ and that of the up-type quark is set to $m_{u 4}=450 \mathrm{GeV}$ [42].

The inclusion of two additional heavy fourth-generation quarks in the SM4 enhances the $H g g$ coupling significantly over the SM and, therefore, increases the $g g \rightarrow H$ production cross section. Although the larger $\mathrm{Hgg}$ coupling also increases the partial decay width for $H \rightarrow g g$, the $H \rightarrow W W$ decay mode continues to dominate over the loop-mediated decays for $M_{H}>135 \mathrm{GeV}$. These facts make the search for a Higgs boson produced via gluon fusion with subsequent decay to $W W, g g \rightarrow H \rightarrow W W$, the most sensitive search for the Higgs boson in the SM4 model. Table 9.3 shows the $\sigma(g g \rightarrow H) \times \mathcal{B}(H \rightarrow W W)$ for each of low-mass and high-mass scenarios in the SM4 compared to that of the SM. The $g g \rightarrow H$ cross section is calculated at the NNLO approximation [42] and the $H \rightarrow W W$ branching fraction is calculated using HDECAY [81] modified to include fourth-generation fermions [39].

\begin{tabular}{||c|c|c|c||}
\hline \hline \multicolumn{4}{|c||}{$\boldsymbol{\sigma}(\boldsymbol{g g} \rightarrow \boldsymbol{H}) \times \mathcal{B}(\boldsymbol{H} \rightarrow \boldsymbol{W} \boldsymbol{W})(\mathbf{f b})$} \\
\hline$M_{H}(\mathrm{GeV})$ & SM4LM & SM4HM & SM \\
\hline 110 & 344.62 & 344.62 & 66.78 \\
115 & 546.98 & 546.61 & 105.42 \\
120 & 778.90 & 778.90 & 153.34 \\
125 & 1071.20 & 1071.20 & 205.05 \\
130 & 1379.15 & 1379.15 & 257.08 \\
135 & 1667.69 & 1667.69 & 302.57 \\
140 & 1989.44 & 1989.44 & 337.98 \\
145 & 2222.18 & 2222.18 & 362.16 \\
150 & 2474.18 & 2474.18 & 376.83 \\
155 & 2731.97 & 2737.19 & 385.26 \\
160 & 3055.84 & 3056.19 & 392.96 \\
165 & 2930.84 & 3033.90 & 368.35 \\
170 & 2546.02 & 2753.46 & 331.96 \\
175 & 2239.78 & 2469.23 & 296.69 \\
180 & 1949.59 & 2172.73 & 260.21 \\
185 & 1569.91 & 1762.75 & 212.77 \\
190 & 1324.59 & 1479.13 & 179.21 \\
195 & 1148.53 & 1295.75 & 156.85 \\
200 & 1027.00 & 1153.72 & 140.12 \\
\hline \hline
\end{tabular}

Table 9.3: The $\sigma(g g \rightarrow H) \times \mathcal{B}(H \rightarrow W W)$ for each of the low-mass (SM4LM) and high-mass (SM4HM) scenarios of the fourth generation fermion models and the SM. 
Our search for the SM4 Higgs boson in the $\ell \nu j j$ final state is performed for Higgs mass hypotheses in the range $110 \leq M_{H} \leq 200 \mathrm{GeV}$ considering only the $g g \rightarrow H \rightarrow W W$ signal process. Since the sensitivity of high $b$-tag channels (one-tight $b$-tag and two $b$-tag) to the $g g \rightarrow H \rightarrow W W$ signal process in the low mass region is very limitted, we only use events in zero and one-loose $b$-tag categories for the search in the range $110 \leq M_{H} \leq 150 \mathrm{GeV}$. Events in all $b$-tag categories are used for the search in the high mass region $155 \leq M_{H} \leq 200 \mathrm{GeV}$. To set the upper limit on the $\sigma(g g \rightarrow H) \times \mathcal{B}(H \rightarrow W W)$ in the SM4, we apply the same analysis steps as for our SM Higgs search, but scale both the $g g \rightarrow H$ cross sections and $H \rightarrow W W$ branching ratio to account for fourth-generation effects. The distributions of the RF discriminants for the SM4 Higgs boson search at $M_{H}=125 \mathrm{GeV}$ for the high-mass scenario in $H \rightarrow W W \rightarrow \mu \nu j j$ analysis using events with two and three jets in the zero $b$-tag and the one-loose $b$-tag categories are shown in Figs. 9.6 and 9.7. Figure 9.8 shows the distributions of the RF discriminants for the SM4 Higgs boson search at $M_{H}=165 \mathrm{GeV}$ for the high-mass scenario in $H \rightarrow W W \rightarrow \mu \nu j j$ analysis.
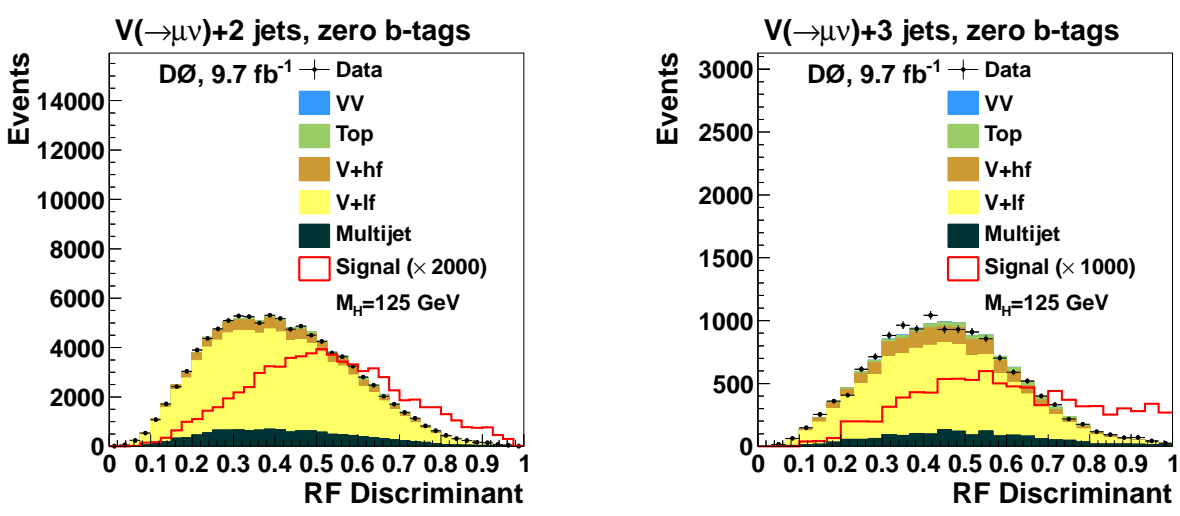

Figure 9.6: Distributions of the RF discriminants for the SM4 Higgs boson search at $M_{H}=$ $125 \mathrm{GeV}$ in zero $b$-tag events with two jets (left) or three jets (right) for the high-mass scenario.

The upper limits on $\sigma(g g \rightarrow H) \times \mathcal{B}(H \rightarrow W W)$ obtained from $H \rightarrow W W \rightarrow \mu \nu j j$ channel and from combining all channels in $\ell \nu j j$ analysis are shown as functions of the Higgs boson mass in Fig. 9.9, along with the theory predictions for fourth-generation models in the low- and high-mass scenarios. In the low-mass scenario, our combined search in $\ell \nu j j$ analysis excludes a SM4 Higgs boson with a mass in the range $150<M_{H}<188 \mathrm{GeV}$ at the $95 \%$ C.L. In the high-mass scenario, the mass range $150<M_{H}<190 \mathrm{GeV}$ is excluded [10]. 

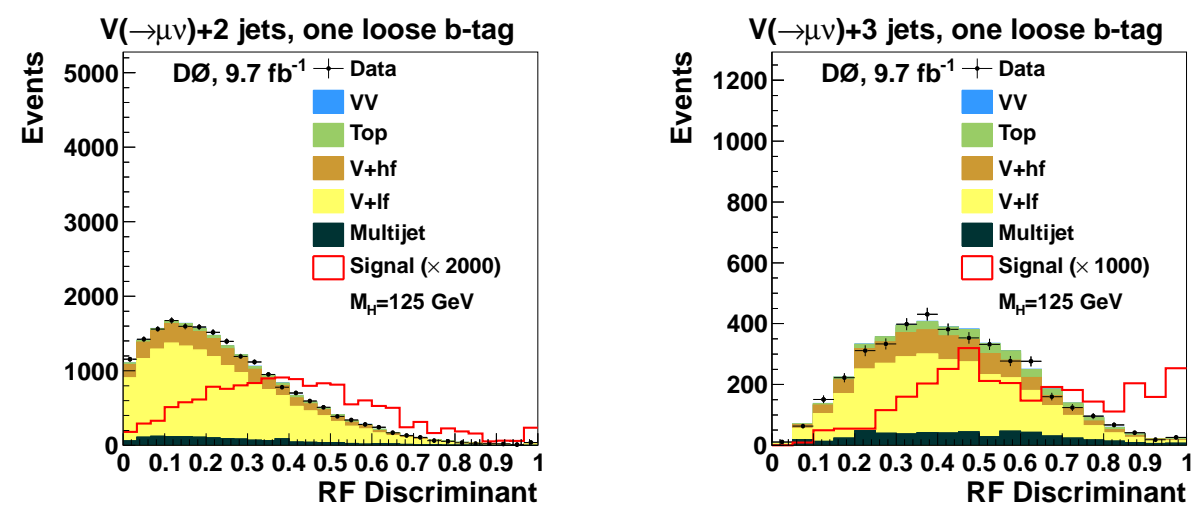

Figure 9.7: Distributions of the RF discriminants for the SM4 Higgs boson search at $M_{H}=$ $125 \mathrm{GeV}$ in one-loose $b$-tag events with two jets (left) or three jets (right) for the high-mass scenario.
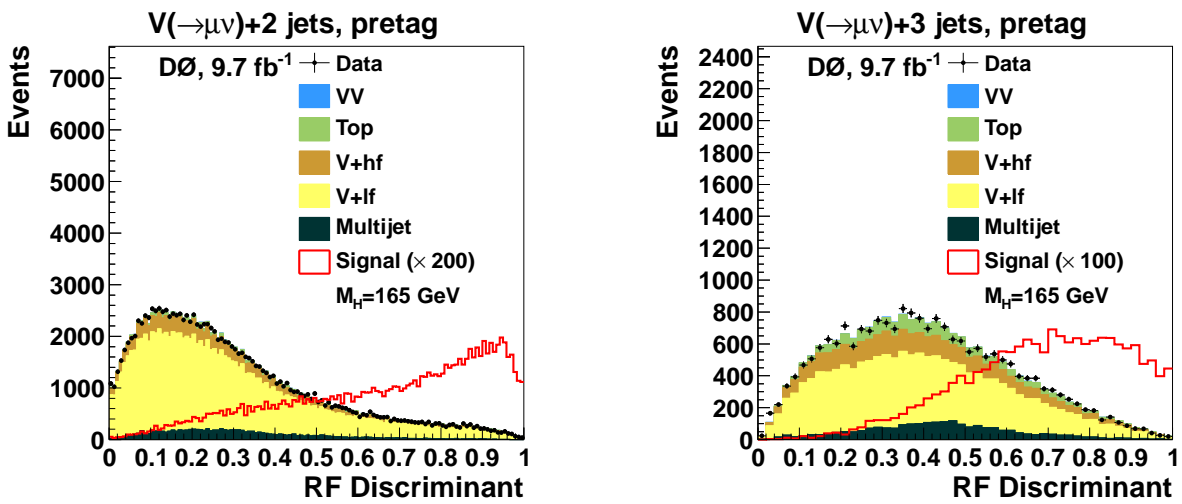

Figure 9.8: Distributions of the RF discriminants for the SM4 Higgs boson search at $M_{H}=$ $165 \mathrm{GeV}$ in all $b$-tag events with two jets (left) or three jets (right) for the high-mass scenario.
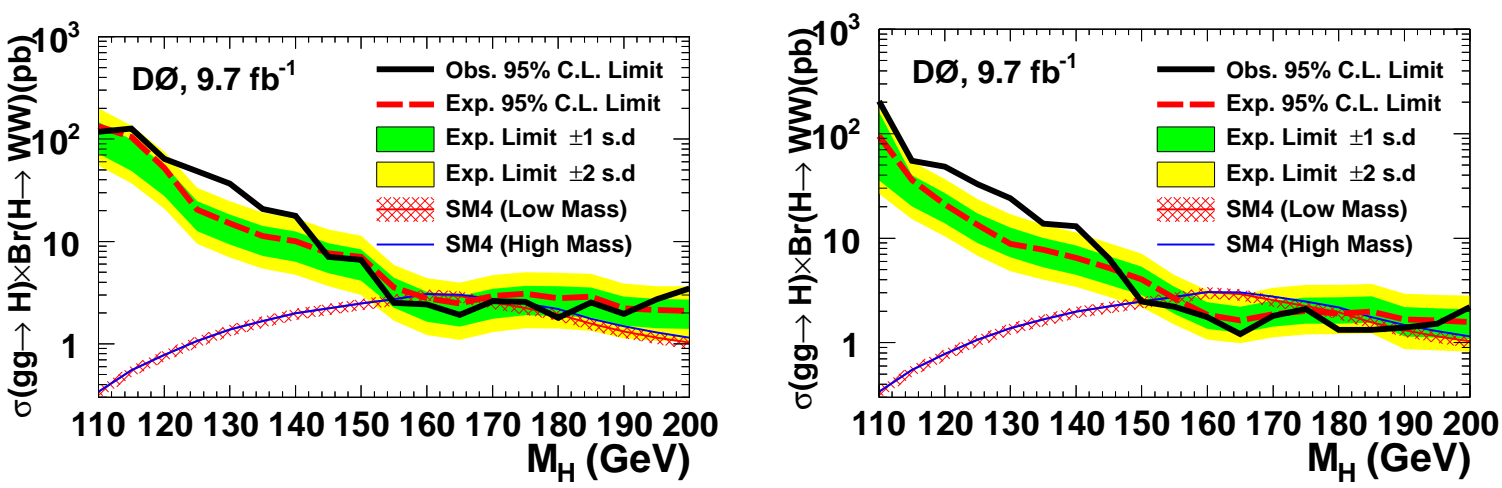

Figure 9.9: Observed and expected limits on the $\sigma(g g \rightarrow H) \times \mathcal{B}(H \rightarrow W W)$ at 95\% C.L. for the $H \rightarrow W W \rightarrow \mu \nu j j$ channel (left) and for all channels in $\ell \nu j j$ analysis combined (right). The green and yellow shaded bands indicate, respectively, the one and two s.d. probability regions in which the expected limits fluctuate in the absence of signal. Theoretical predictions for SM4 in the low- and high-mass scenarios are shown with blue and red lines, respectively. The hatched band indicates the theoretical uncertainty associated with the SM4 low-mass scenario. 


\section{Fermiophobic Higgs Model Interpretation}

In the Fermiophobic Higgs model, the Higgs boson does not couple to fermions at the tree level, but behaves like the SM Higgs boson in coupling to bosons. The zero tree-level couplings of the Higgs boson to fermions forbids the direct decay to fermions $(H \rightarrow f f)$ and suppresses the production cross section via gluon fusion $(g g \rightarrow H)$. The decays to two weak bosons and two photons are enhanced accounting for nearly the entire decay width of the Higgs boson. Table 9.4 shows the branching ratios for $H \rightarrow V V$ decays in the FHM in comparison to that in the SM.

\begin{tabular}{||c|c|c|c|c||}
\hline \hline$M_{H}(\mathrm{GeV})$ & \multicolumn{2}{|c|}{$\mathcal{B}(H \rightarrow W W)$} & \multicolumn{2}{c||}{$\mathcal{B}(H \rightarrow Z Z)$} \\
& FHM & SM & FHM & SM \\
\hline 100 & 0.7353 & 0.011 & 0.0763 & 0.001 \\
105 & 0.8157 & 0.024 & 0.0733 & 0.002 \\
110 & 0.8527 & 0.048 & 0.0788 & 0.004 \\
115 & 0.8664 & 0.086 & 0.0887 & 0.009 \\
120 & 0.8694 & 0.143 & 0.0993 & 0.016 \\
125 & 0.8684 & 0.216 & 0.1087 & 0.027 \\
130 & 0.8667 & 0.305 & 0.1160 & 0.040 \\
135 & 0.8662 & 0.403 & 0.1205 & 0.055 \\
140 & 0.8682 & 0.504 & 0.1214 & 0.069 \\
145 & 0.8741 & 0.603 & 0.1178 & 0.079 \\
150 & 0.8861 & 0.699 & 0.1077 & 0.083 \\
155 & 0.9086 & 0.796 & 0.0871 & 0.074 \\
160 & 0.9512 & 0.909 & 0.0466 & 0.042 \\
165 & 0.9754 & 0.960 & 0.0236 & 0.022 \\
170 & 0.9748 & 0.965 & 0.0246 & 0.024 \\
175 & 0.9663 & 0.958 & 0.0332 & 0.032 \\
180 & 0.9386 & 0.932 & 0.0609 & 0.060 \\
185 & 0.8479 & 0.844 & 0.1517 & 0.150 \\
190 & 0.7881 & 0.786 & 0.2116 & 0.209 \\
195 & 0.7590 & 0.757 & 0.2407 & 0.239 \\
200 & 0.7423 & 0.741 & 0.2575 & 0.256 \\
\hline \hline
\end{tabular}

Table 9.4: The branching ratio of $H \rightarrow V V$ decays in the FHM and the SM.

Our $\ell \nu j j$ analysis here seeks a FHM Higgs boson produced via either the vector-bosonfusion (VBF) or $W / Z$ associated production process and subsequently decaying to $W W$ or $Z Z$. We perform similar analysis steps as for the SM Higgs search, but set the contribution from $g g \rightarrow H$ production to zero and scale the contributions from fermiophobic signal processes, $V B F \rightarrow H \rightarrow V V$ and $V H \rightarrow V V V$ (where $V \equiv W$ or $Z$ ) to reflect the predicted rate in the FHM. The contribution of fermiophobic signal processes in high $b$-tag categories (one-tight $b$-tag and two $b$-tag) to the search at lower Higgs masses is very limited and, therefore, is neglected. Only events with two or more jets in zero and one loose b-tag 
categories are used in the search for $110 \leq M_{H} \leq 150 \mathrm{GeV}$. Events in all $b$-tag categories are examined for the search in high mass region $155 \leq M_{H} \leq 200 \mathrm{GeV}$. To set the upper limits on the Higgs boson production cross section multiplied by the branching ratio in the FHM, we retrain RF discriminant for fermiophobic signal processes for each sub-channel. The distributions of RF discriminants for the $H \rightarrow W W \rightarrow \mu \nu j j$ channel at the Higgs mass of $125 \mathrm{GeV}$ are shown in Figs. 9.10 and 9.11.
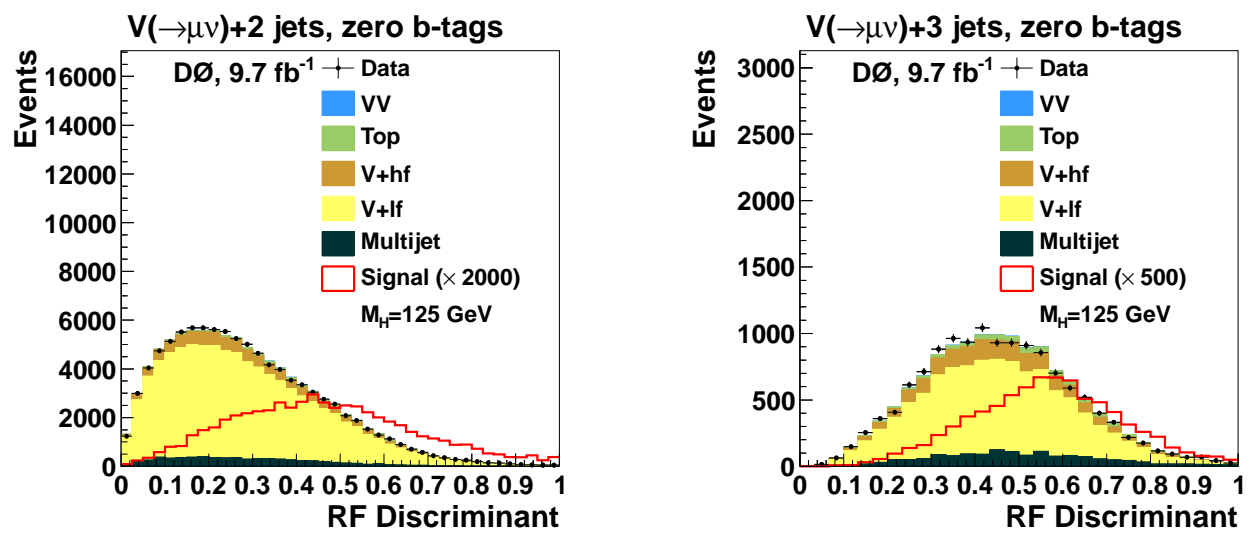

Figure 9.10: Distributions of the RF discriminants for the FHM Higgs boson search at $M_{H}=125 \mathrm{GeV}$ in zero $b$-tag events with two jets (left) or three jets (right).
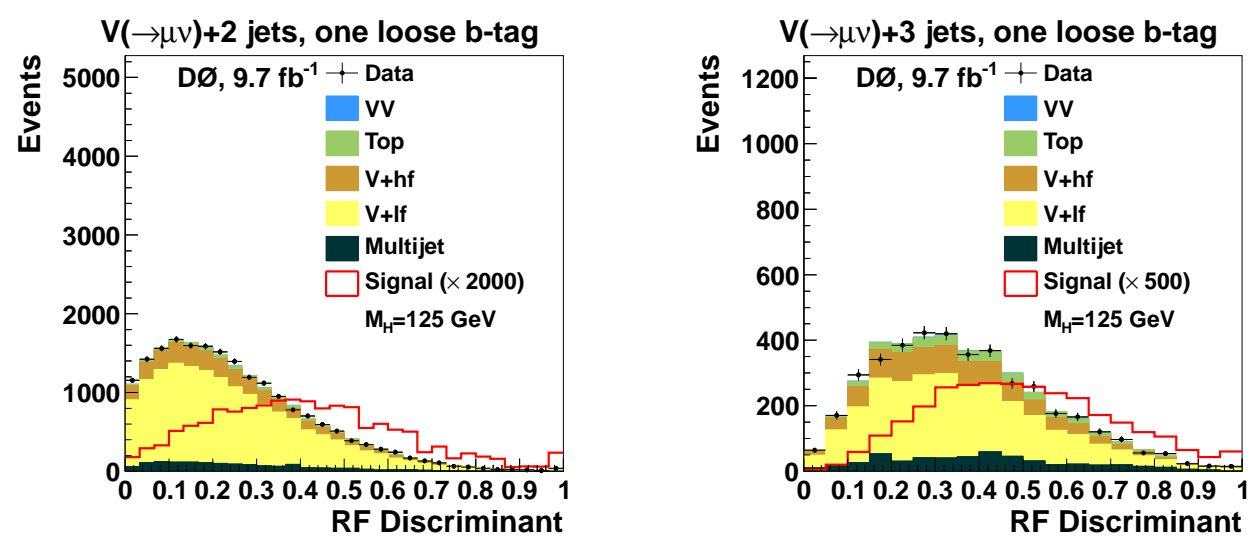

Figure 9.11: Distributions of the RF discriminants for the FHM Higgs boson search at $M_{H}=125 \mathrm{GeV}$ in one-loose $b$-tag events with two jets (left) or three jets (right).

The upper limits on Fermiophobic Higgs boson production are calculated at 95\% C.L. as a function of the Higgs boson mass for the $H \rightarrow W W \rightarrow \mu \nu j j$ channel and presented in units of the FHM prediction in Fig. 9.12 and Table 9.5. The resulting limits for the combined search in the $\ell \nu j j$ analysis are also shown in Fig. 9.12. 

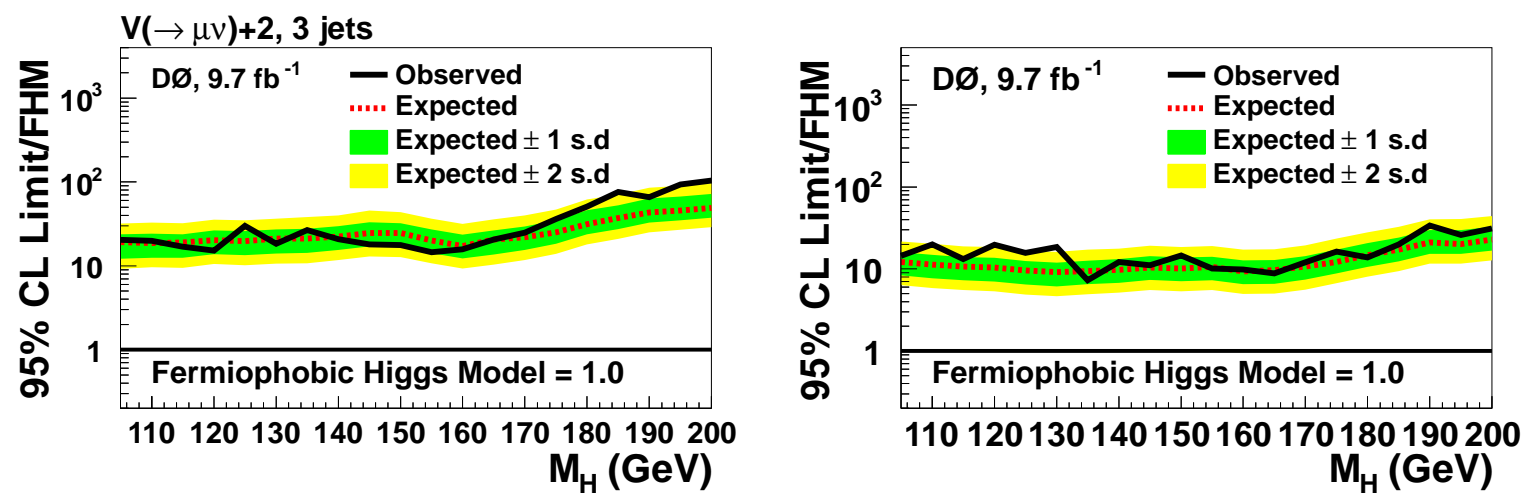

Figure 9.12: Observed and expected upper limits at 95\% C.L. on fermiophobic Higgs boson production as a function of its mass for the $H \rightarrow W W \rightarrow \mu \nu j j$ channel (left) and all channels in the $\ell \nu j j$ analysis combined (right). The limits are presented in the unit of the FHM cross section. The green and yellow shaded bands indicate, respectively, the one and two s.d. probability regions in which the expected limits fluctuate in the absence of signal.

\begin{tabular}{||c|cccccccccccc||}
\hline \hline & \multicolumn{10}{c||}{ Upper limits $\left(\boldsymbol{\sigma}_{\mathbf{L M}} / \boldsymbol{\sigma}_{\mathrm{FHM}}\right)$} & for $\boldsymbol{H} \rightarrow \boldsymbol{W} \boldsymbol{W} \rightarrow \boldsymbol{\mu} \boldsymbol{\nu} \boldsymbol{j} \boldsymbol{j}$ channel & \\
\hline$M_{H}(\mathrm{GeV})$ & 100 & 105 & 110 & 115 & 120 & 125 & 130 & 135 & 140 & 145 & 150 \\
\hline Expected & 179.9 & 19.2 & 18.7 & 18.9 & 20.2 & 19.6 & 21.1 & 21.1 & 22.3 & 24.7 & 24.7 \\
Observed & 186.1 & 20.5 & 19.9 & 16.9 & 15.2 & 30.1 & 18.3 & 26.8 & 20.7 & 18.1 & 17.7 \\
\hline \hline$M_{H}(\mathrm{GeV})$ & 155 & 160 & 165 & 170 & 175 & 180 & 185 & 190 & 195 & 200 & \\
\hline Expected & 20.1 & 17.2 & 20.5 & 21.9 & 25.1 & 31.4 & 37.3 & 43.2 & 45.6 & 49.2 & \\
Observed & 14.6 & 15.7 & 20.7 & 24.79 & 36.2 & 50.9 & 76.3 & 65.8 & 93.6 & 103.9 & \\
\hline \hline
\end{tabular}

Table 9.5: The expected and observed 95\% C.L. limits, as a function of the Higgs boson mass $M_{H}$, presented as ratios of production cross section times branching fraction to the expected FHM prediction.

\subsection{Results of the SM Higgs Boson Searches in $Z H \rightarrow \ell \ell b b$ Analysis}

\subsubsection{Upper Limits on the SM Higgs Boson Production}

We use the global RF output distributions of the four sub-samples for the single $b$-tag and double $b$-tag in the $t \bar{t}$-depleted and $t \bar{t}$-enriched regions in $Z H \rightarrow \ell \ell b b$ analysis (Sec. 7.2) along with the corresponding systematic uncertainties to extract the upper limits for the SM Higgs boson production in the region $90 \leq M_{H} \leq 150 \mathrm{GeV}$, in steps of $5 \mathrm{GeV}$ [11]. Figure 9.13 shows the distributions of the LLR for observed data (LLR ${ }_{\mathrm{OBS}}$ ) and the median LLR values corresponding to background-only $\left(\operatorname{LLR}_{\mathrm{B}}\right)$ and signal-plus-background $\left(\mathrm{LLR}_{\mathrm{S}+\mathrm{B}}\right)$ hypotheses. For $M_{H}>120 \mathrm{GeV}$, the observed LLR favors the signal-plus-background hypothesis. The upper limits at the $95 \%$ C.L. on the product of the $Z H \rightarrow \ell \ell b b$ production cross section and branching ratio are expressed as a ratio to the SM prediction and shown in Fig. 9.13 and Table 9.6. At $M_{H}=125 \mathrm{GeV}$, the observed and expected limits for $Z H \rightarrow \ell \ell b b$ analysis are $7.1 \times \sigma_{\mathrm{SM}}$ and $5.1 \times \sigma_{\mathrm{SM}}$, respectively. 

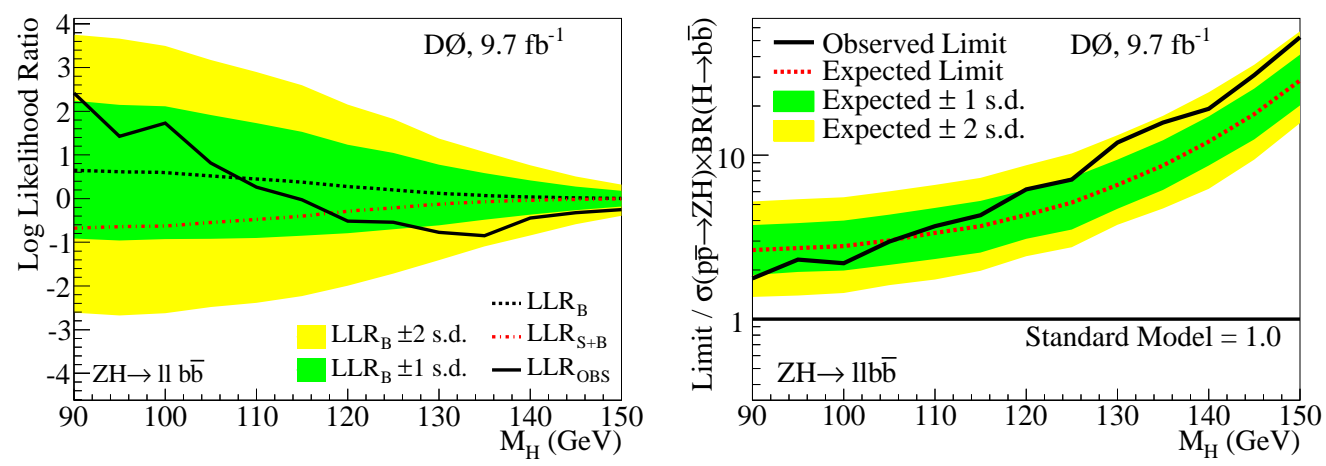

Figure 9.13: (Left) Observed LLR and median LLR values for $\mathrm{S}+\mathrm{B}$ and $B$ hypotheses as functions of $M_{H}$ for the $S+B$ and $B$ hypotheses, along with the \pm 1 and \pm 2 s.d. bands for the $B$ hypotheses, for the $Z H \rightarrow \ell \ell b b$ analysis. (Right) Expected and observed limits at the $95 \%$ C.L. on the $Z H \rightarrow \ell^{+} \ell^{-} b \bar{b}$ production cross section, relative to the SM prediction.

\begin{tabular}{||lccccccccccccc||}
\hline \hline \multicolumn{10}{|c||}{ Upper limits $\left(\boldsymbol{\sigma}_{\mathbf{L M}} / \boldsymbol{\sigma}_{\mathbf{S M}}\right)$ for $\boldsymbol{Z} \boldsymbol{H} \boldsymbol{\rightarrow} \boldsymbol{\ell} \boldsymbol{\ell} \boldsymbol{b} \boldsymbol{b}$ analysis } \\
\hline \hline$M_{H}(\mathrm{GeV})$ & 90 & 95 & 100 & 105 & 110 & 115 & 120 & 125 & 130 & 135 & 140 & 145 & 150 \\
\hline Expected & 2.6 & 2.7 & 2.8 & 3.0 & 3.4 & 3.7 & 4.3 & 5.1 & 6.6 & 8.7 & 12 & 18 & 29 \\
Observed & 1.8 & 2.3 & 2.2 & 3.0 & 3.7 & 4.3 & 6.2 & 7.1 & 12 & 16 & 19 & 31 & 53 \\
\hline \hline
\end{tabular}

Table 9.6: The expected and observed upper limits at the 95\% C.L. on the SM Higgs boson production cross section for $Z H \rightarrow \ell^{+} \ell^{-} b \bar{b}$, expressed as a ratio to the SM cross section.

\subsubsection{RF Technique Validation with Matrix Element Results}

ME discriminants for the $Z H \rightarrow \ell \ell b b$ analysis are derived based on physically motivated calculation and, therefore, can be used as a valuable cross check for the more "black box" approach of deriving the RF discriminants. To validate our RF technique, we determine the upper limits on the product of the $Z H \rightarrow \ell \ell b b$ production cross section and branching ratio $\sigma_{(\mathrm{p} \overline{\mathrm{p}} \rightarrow \mathrm{ZH})} \times \mathcal{B}_{(\mathrm{H} \rightarrow \mathrm{b} \overline{\mathrm{b}})}$ for a tested data set of $3.1 \mathrm{fb}^{-1}$ in $Z H \rightarrow \mu^{-} \mu^{+} b \bar{b}$ channel using the ME discriminant and compare the resulting limits to that obtained using the RF discriminants.

Using ME discriminants as inputs for the limit-setting procedure, we obtain the LLR distributions and the upper limits for the tested data set, shown as functions of the Higgs boson mass $M_{H}$ in Fig. 9.14. The upper limits determined from the RF discriminants for the same data set are shown in comparison with the resulting limits obtained from using the ME discriminants in Fig. 9.15. The compatibility of the signal sensitivities extracted from the ME discriminants and RF discriminants verifies the performance of the random forest for the $Z H \rightarrow l l b b$ channel.

In order to improve signal sensitivity in the $Z H \rightarrow l l b b$ analysis, we combine the ME and $\mathrm{RF}$ techniques by feeding the calculated ME probabilities into a random forest as additional input variables. A comparison of the expected limits obtained using RF classifiers which are trained with or without using the ME probabilities as RF input variables are shown in Fig. 9.16. As a preliminary result, we observe an improvement of around $5 \%$ in expected sensitivity for the Higgs boson signal by combining the ME and RF techniques. 

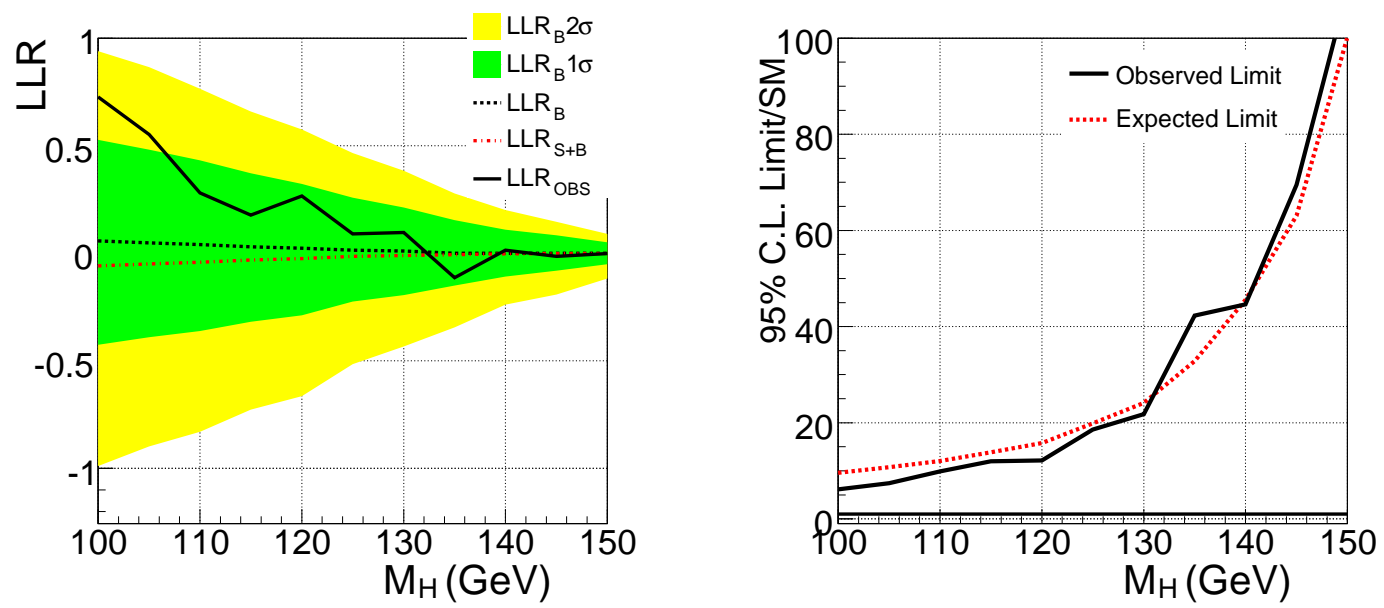

Figure 9.14: The LLR distributions (left) and the upper limits (right) determined from ME discriminants. The LLR plot shows the median LLR for the background-only expectation $\left(\mathrm{LLR}_{\mathrm{B}}\right)$, the signal-plus-background expectation $\left(\mathrm{LLR}_{\mathrm{S}+\mathrm{B}}\right)$ and the observed data (LLR $\left.\mathrm{OBS}_{\mathrm{S}}\right)$. The green and yellow bands are the \pm 1 s.d. and \pm 2 s.d. on the background-only expectation. The observed (solid black line) and expected (dotted red line) limits on the $Z H \rightarrow \ell \ell b b$ production are expressed relative to the SM rate.

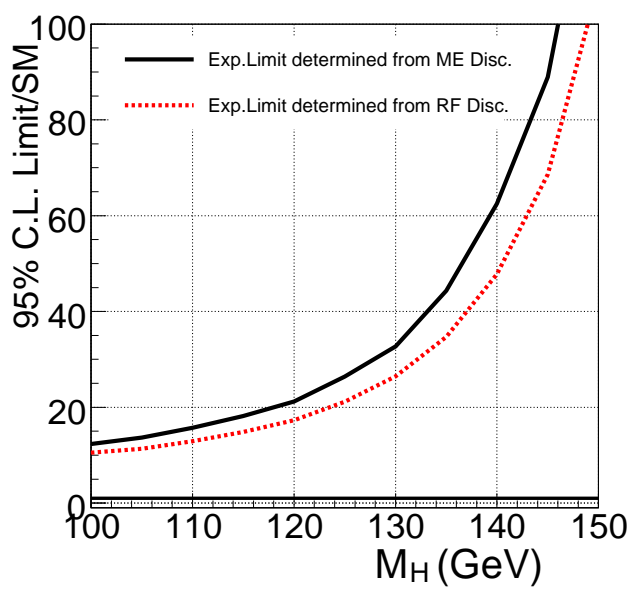

Figure 9.15: The expected limits on the $Z H \rightarrow \ell \ell b b$ production obtained using the $\mathrm{RF}$ discriminants (red) and ME discriminants (black). The limits are presented in the unit of the SM rate.

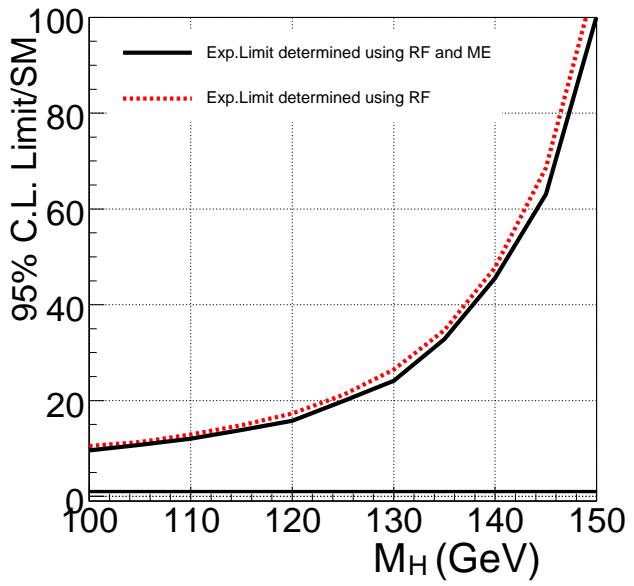

Figure 9.16: The expected limits on the $Z H \rightarrow \ell \ell b b$ production obtained from using only the RF technique (black) and from combining the $\mathrm{ME}$ and $\mathrm{RF}$ techniques (red). The limits are presented in the unit of the SM rate. 


\subsection{Combined Results from DØ and Tevatron Higgs Boson Searches}

The direct searches for the Higgs boson in $p \bar{p}$ collisions at the Tevatron are organized into analysis subchannels comprising different production, decay, and final state particle configurations, in order to maximize the sensitivity for each particular Higgs boson production and decay mode. Results of the SM Higgs boson searches focusing on the $H \rightarrow b \bar{b}, H \rightarrow W^{+} W^{-}$, $H \rightarrow Z Z, H \rightarrow \tau^{+} \tau^{-}$, and $H \rightarrow \gamma \gamma$ decay modes are then combined for the D $\varnothing$ Collaboration and for both $\mathrm{D} \varnothing$ and CDF Collaborations $[12,13]$. The combinations are also interpreted in fermiophobic and fourth generation models.
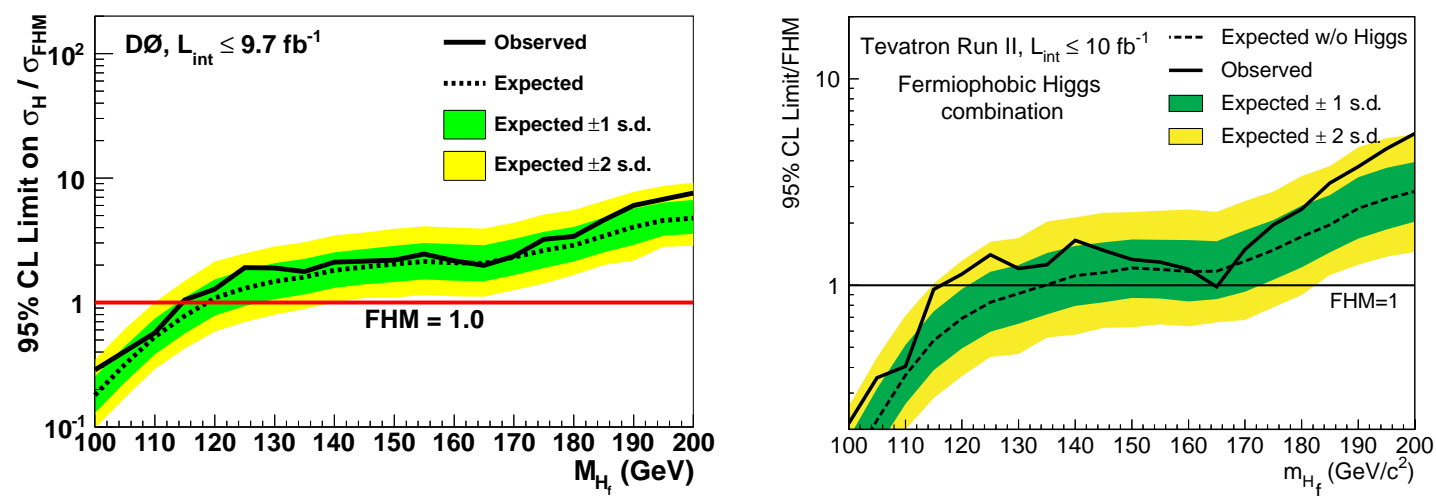

Figure 9.17: Observed and expected upper limits at 95\% C.L. on the fermiophobic Higgs boson production as function of Higgs boson mass for the D $\varnothing$ combination (left) and the Tevatron combination (right). The limits are presented in units of the FHM cross section. The green and yellow shaded bands indicate, respectively, the one and two s.d. probability regions in which the expected limits fluctuate in the absence of signal.

Figure 9.17 shows the upper limits on the fermiophobic Higgs boson production at $95 \%$ C.L. in the mass range of $100 \leq M_{H} \leq 200 \mathrm{GeV}$ for the $\mathrm{D} \varnothing$ and Tevatron combinations. The $\mathrm{D} \varnothing$ combination excludes a fermiophobic Higgs boson with a mass below $114 \mathrm{GeV}$. Integrating results of searches by both $\mathrm{D} \varnothing$ and CDF Collaborations, the Tevatron combination excludes a fermiophobic Higgs boson in the mass range of $100-116 \mathrm{GeV}$.

In the presence of a fourth sequential generation of fermions, the $95 \%$ C.L. upper limits on the cross section times branching ratio $\sigma(g g \rightarrow H) \times \mathcal{B}(H \rightarrow W W)$ along with the theory predictions for fourth-generation models in the low- and high-mass scenarios from the DØ combination and from the Tevatron combination are shown in Fig. 9.18. The combined result of searches by the D $\varnothing$ Collaboration excludes a SM4 Higgs boson in the mass ranges of $125<M_{H}<218 \mathrm{GeV}$ and $125<M_{H}<228$ at $95 \%$ C.L. in the low-mass and high-mass scenarios, respectively. Combination of $\mathrm{D} \varnothing$ and CDF results excludes a SM4 Higgs boson at $95 \%$ C.L in the mass range $121<M_{H}<225 \mathrm{GeV}\left(125<M_{H}<232\right)$ in the low-mass (high-mass) scenario. 

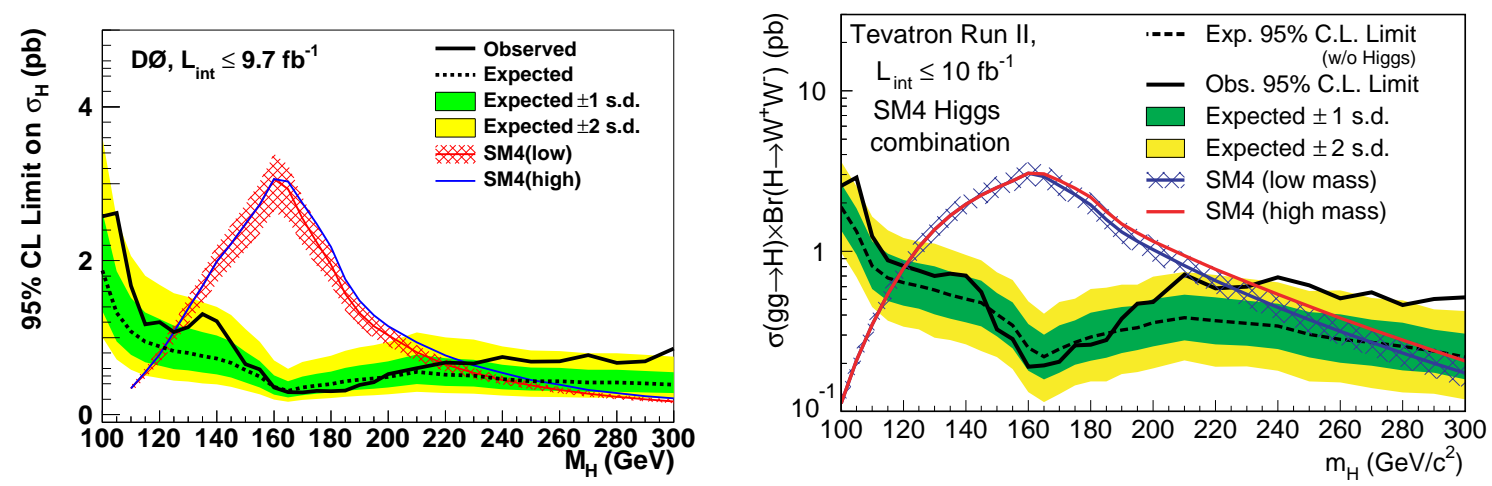

Figure 9.18: Observed and expected limits on the $\sigma(g g \rightarrow H) \times \mathcal{B}(H \rightarrow W W)$ at 95\% C.L. for the $\mathrm{D} \varnothing$ combination (left) and the Tevatron combination (right). The green and yellow shaded bands indicate, respectively, the one and two s.d. probability regions in which the expected limits fluctuate in the absence of signal. Theoretical predictions for SM4 in the low- and high-mass scenarios are shown with blue and red lines. The hatched band indicates the theoretical uncertainty associated with the SM4 low-mass scenario.

The LLR distributions obtained from combinations of searches for the SM Higgs boson conducted by the $\mathrm{D} \varnothing$ Collaboration and by both $\mathrm{D} \varnothing$ and CDF Collaborations are shown in Fig. 9.19 as functions of $M_{H}$. The upper limits on the SM Higgs boson production for the DØ and Tevatron combinations are displayed in Fig. 9.20. The observed data for each combination are consistent with the background-only hypothesis at masses smaller than $\approx 110 \mathrm{GeV}$ and above $\approx 145 \mathrm{GeV}$. Intersections of the observed limit curves with the $\mathrm{SM}=1$ line in the limits plots are used to quote ranges of Higgs boson masses that are excluded in the SM. The result of DØ combination excludes the SM Higgs boson at 95\% C.L. in the mass ranges $90<M_{H}<101 \mathrm{GeV}$ and $157<M_{H}<178 \mathrm{GeV}$. The Tevatron result excludes the SM Higgs boson at the $95 \%$ C.L. in the mass ranges from 90 to $109 \mathrm{GeV}$ and from 149 to $182 \mathrm{GeV}$.

For the mass range $115<M_{H}<140 \mathrm{GeV}$, the data recorded by the DØ detector exhibit an excess up to two s.d. with respect to the SM background-only expectation, while Tevatron combined result shows a significant excess above two standard deviations in the data over the expected background. Additional distributions of median LLR and the upper limits corresponding to the hypothesis that a SM Higgs boson is present with $M_{H}=125 \mathrm{GeV}$ are also shown in Figs. 9.19 and 9.20. These signal-injected LLR and limit curves are consistent with the distributions of the observed LLR and observed limits. 

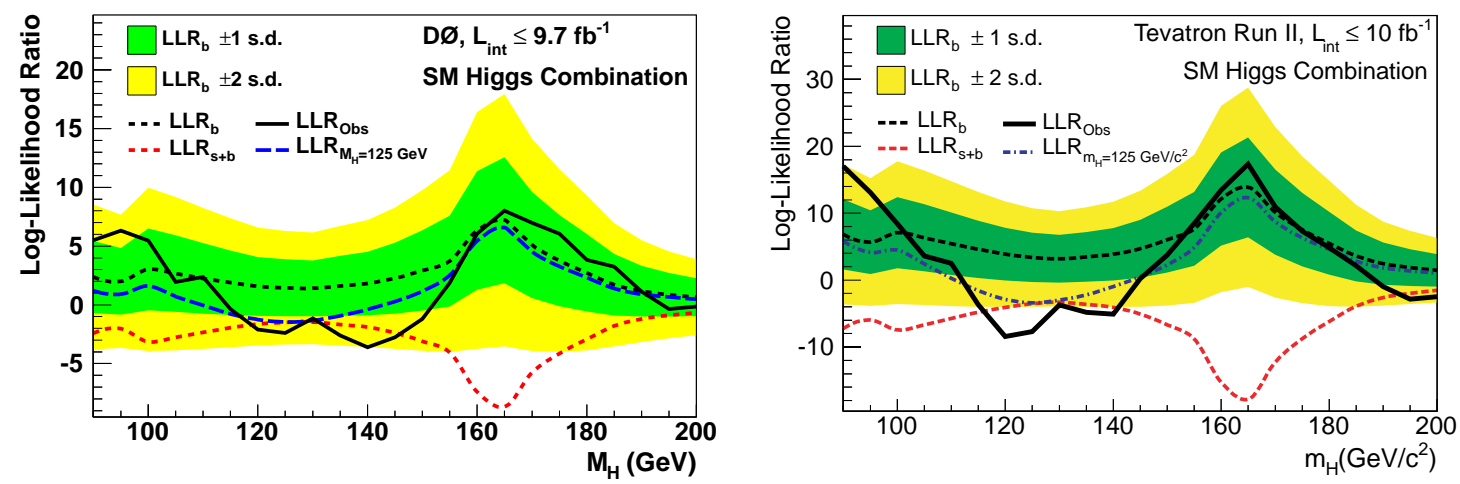

Figure 9.19: The log-likelihood ratio LLR as a function of Higgs boson mass for the D $\varnothing$ combination (left) and full Tevatron combination (right) of SM Higgs boson searches. The solid line shows the observed LLR values, the black dashed line shows the median expectation assuming no Higgs boson signal is present. The green and yellow shaded bands correspond, respectively, to the regions encompassing one and two s.d. fluctuations around the background-only expectation. The red dashed line shows the median expectation assuming a SM Higgs boson signal is present at each value of $M_{H}$. The blue dashed line shows the median expected LLR assuming the SM Higgs boson is present at $M_{H}=125 \mathrm{GeV}[10,13]$.
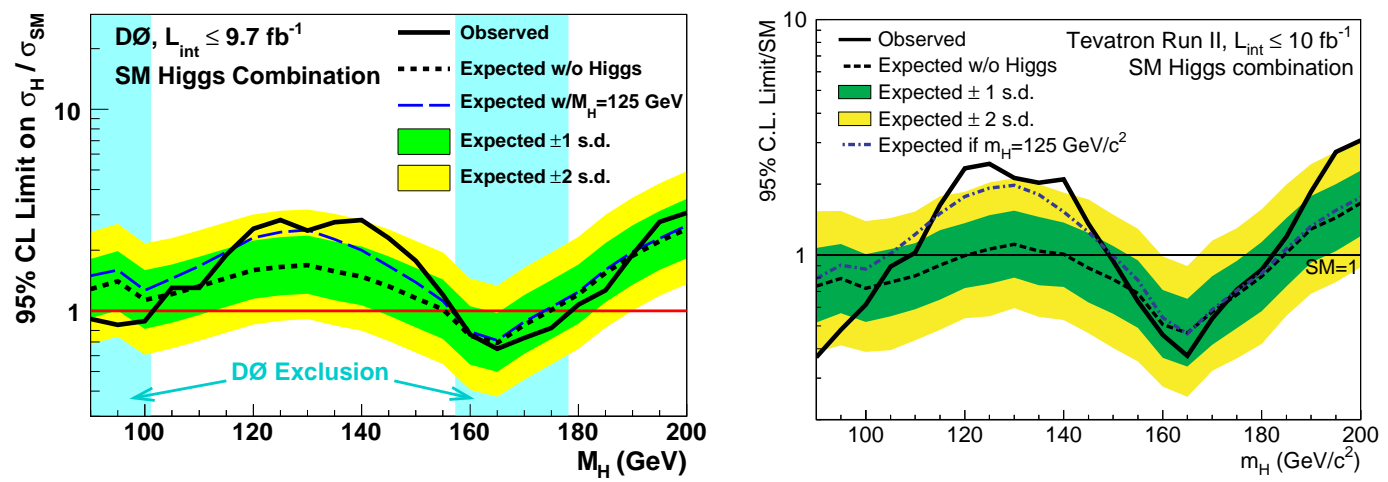

Figure 9.20: Observed and median expected upper limits at 95\% C.L on the cross section of the SM Higgs boson for the DØ combination (left) and full Tevatron combination (right). The limits are expressed as multiples of the SM prediction of the Higgs boson cross section for each value of $M_{H}$ in the mass range $90-200 \mathrm{GeV}$. The green and yellow shaded bands indicate, respectively, the one and two s.d probability regions in which the limits are expected to fluctuate in the absence of signal. The blue dashed line shows median expected limits assuming the SM Higgs boson is present at $M_{H}=125 \mathrm{GeV}[10,13]$. 
To quantify the significance of the excess in the data over the background prediction for the full Tevatron combination, we calculate the $p$-value with respect to the backgroundonly hypothesis at each hypothesized Higgs boson mass. Figure 9.21 displays the observed $p$-value, along with the expected $p$-value assuming a SM Higgs boson signal is present, for each value of $M_{H}$. The median expected $p$-value assuming a SM Higgs boson is presented with $M_{H}=125 \mathrm{GeV}$ for a signal strength of 1.0 and 1.5 times the SM prediction are also shown. As seen in Fig. 9.21, the observed $p$-value is close to 0.5 for $145 \leq M_{H} \leq 180$, indicating the agreement between the data and the background-only hypothesis. For the mass range $115<M_{H}<140$, the observed $p$-value drops significantly. The observed $p$-value at $M_{H}=125 \mathrm{GeV}$, which expresses the probability that background would fluctuate up to the excess seen in the data at this mass point, corresponds 3.0 standard deviations. This result of the Tevatron combination is consistent with the mass of the Higgs boson observed at the $\mathrm{LHC}[5,6]$.

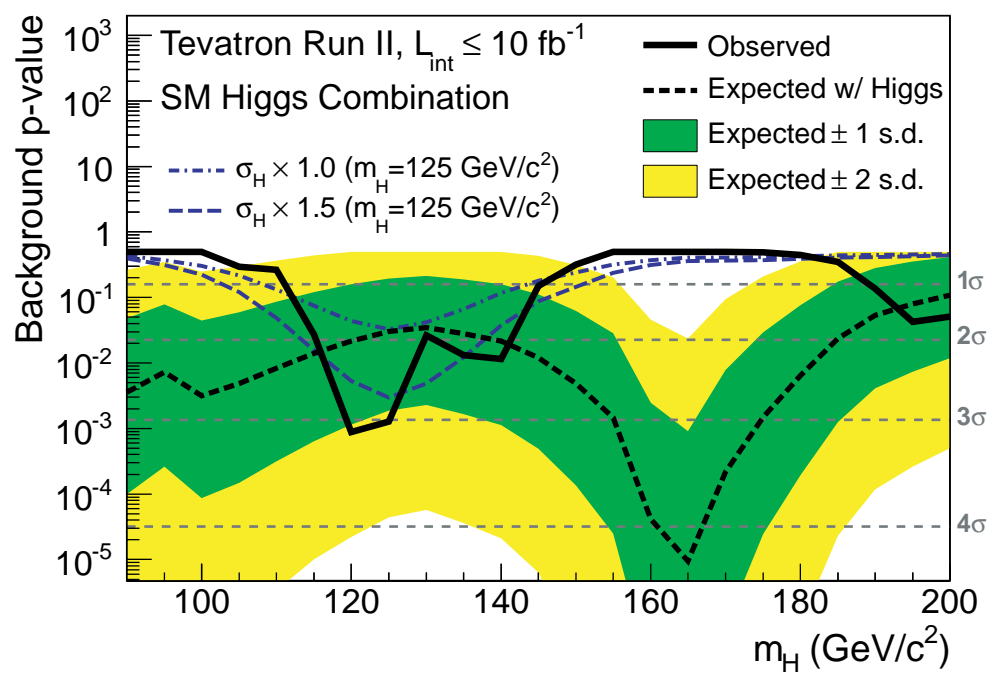

Figure 9.21: The $p$-values calculated separately at each value of $M_{H}$ for the full Tevatron combination. The solid black line displays the observed $p$-value and the dotted black line shows the median expected values assuming a SM signal is present. The associated green and yellow shaded bands indicate the one and two s.d. fluctuations of possible experimental outcomes under this scenario. The blue lines show the median expected $p$-values assuming the SM Higgs boson is present with $M_{H}=125 \mathrm{GeV}$ at signal strengths of 1.0 times (shortdashed) and 1.5 times (long-dashed) the SM prediction [13]. 


\section{0}

\section{Conclusion}

Searches for SM Higgs boson production in the leptons plus jets final states with a data set corresponding to $9.7 \mathrm{fb}^{-1}$ of $p \bar{p}$ collisions at $\sqrt{s}=1.96 \mathrm{TeV}$ collected by the DØ Experiment are presented in this thesis. The searches are carried out in two independent analyses, accounting for different signal topologies.

The $\ell \nu j j$ analysis investigates events containing at least two jets, a charged lepton and a large missing transverse energy arising from the presence of a neutrino. It comprises searches for three main signal processes, $W H \rightarrow \ell \nu b b, H \rightarrow W W \rightarrow \ell \nu j j$, and $W H \rightarrow W W W \rightarrow$ $\ell \nu j j j j$, in the mass range $90 \leq M_{H} \leq 200 \mathrm{GeV}$. The $Z H \rightarrow \ell \ell b b$ analysis performs a search for the SM Higgs boson produced in association with a $\mathrm{Z}$ boson and subsequently decaying to a bottom and anti-bottom quark pair in the mass range $90 \leq M_{H} \leq 150 \mathrm{GeV}$, using events having a pair of charged leptons and least two jets in the final state.

Searching for the SM Higgs boson in both analyses is challenging due to a small signal expectation and large background contributions at the Tevatron. To focus on each particular Higgs boson production and decay mode as well as the final state particle configuration, the data set of each analysis is divided into independent sub-channels according to lepton flavor, jet multiplicity, and the number and quality of b-tagged jets. Multivariate analyses are optimized for each individual sub-channel to maximize the resolving power for discriminating the Higgs boson signal from backgrounds. In order to obtain the optimal signal sensitivity, advanced statistical tools are applied to control the impact of systematic uncertainties and set the upper limits on the SM Higgs boson production for combined sub-channels, based on the outputs of the MVA.

Discriminants obtained with the Random Forest classifiers are used as inputs for setting the upper limits on the Higgs boson production in the $Z H \rightarrow \ell \ell b b$ analysis and the $H \rightarrow$ $W W \rightarrow \ell \nu j j$ channel in the $\ell \nu j j$ analysis. In order to improve the performance of the RF classifiers in the $Z H \rightarrow \ell \ell b b$ analysis, we employ the kinematic fit and gain an improvement $\sim 15 \%$ in the resolution of the reconstructed dijet mass, which is the most important variable for the RF classifiers. Additionally, to validate the Random Forest technique, we construct the Matrix Element discriminants for a sub-dataset of the $Z H \rightarrow \ell \ell b b$ analysis. The upper 
limits obtained from using the ME discriminants are consistent with the corresponding limits obtained by using the RF discriminants.

Our search results for the SM Higgs boson in the $\ell \nu j j$ and the $Z H \rightarrow \ell \ell b b$ analyses have been published in Refs. [10] and [11]. We set 95\% C.L. upper limits on the SM Higgs boson production cross section in the mass range $90 \leq M_{H} \leq 200 \mathrm{GeV}$ for the $\ell \nu j j$ analysis and in the mass range from 90 to $150 \mathrm{GeV}$ for the $Z H \rightarrow \ell \ell b b$ analysis. The observed (expected) limits at $M_{H}=125 \mathrm{GeV}$ for the $\ell \nu j j$ and the $Z H \rightarrow \ell \ell b b$ analyses are 7.1 (5.1) and 5.8 (4.7) times the SM expectation, respectively. We also interpret the searches in the $\ell \nu j j$ analysis in terms of models containing a fourth generation of fermions, as well as models with a fermiophobic Higgs boson. In these interpretations, we exclude $150<M_{H}<188$ (190) $\mathrm{GeV}$ in the low-mass (high-mass) fourth generation fermion scenario, and provide 95\% C.L limits on the production cross section in the fermiophobic model.

Each of $\ell \nu j j$ and the $Z H \rightarrow \ell \ell b b$ analyses provide inputs to the D $\varnothing$ combination and the overall Tevatron combination. Our combined results of the searches for the SM Higgs boson in leading decay modes $H \rightarrow b \bar{b}, H \rightarrow W^{+} W^{-}, H \rightarrow Z Z, H \rightarrow \tau^{+} \tau^{-}$, and $H \rightarrow \gamma \gamma$ for the mass range $90 \leq M_{H} \leq 200 \mathrm{GeV}$ were published in Refs. [12] and [13]. The results of combined searches interpreted in fermiophobic and fourth generation models are also described in these papers.

The maximum sensitivity to the Higgs boson produced in $p \bar{p}$ collisions was attained from the Tevatron combination. We exclude the Fermiophobic Higgs boson in the mass range $100<M_{H}<116 \mathrm{GeV}$ at the $95 \%$ C.L., and exclude the SM4 Higgs boson in the presence of a fourth sequential generation of fermions in the mass range $121<M_{H}<225 \mathrm{GeV}$ $\left(125<M_{H}<232 \mathrm{GeV}\right)$ in the low-mass (high-mass) scenario at the $95 \%$ C.L. The SM Higgs boson in the mass ranges from 90 to $109 \mathrm{GeV}$ and from 149 to $182 \mathrm{GeV}$ are excluded at $95 \%$ C.L. In the mass range of $M_{H} \approx 115-140 \mathrm{GeV}$, the data recorded at the Tevatron exhibit an excess above the background prediction of more than two standard deviations. At $M_{H}=125 \mathrm{GeV}$, where the largest contribution to the Higgs boson sensitivity at the Tevatron comes from the $H \rightarrow b \bar{b}$ decay mode, we observe an excess in data consistent with the Higgs boson observed at the LHC, at the level of three standard deviations.

The Standard Model insists that the Higgs boson is the unique source of mass for all quarks, leptons and gauge bosons, and its couplings to those fundamental particles are precisely in the ratio of their masses. The ATLAS and CMS Collaborations at the LHC have discovered a Higgs boson with mass near $126 \mathrm{GeV}$ in the bosonic final states, where the Higgs boson decays to $\gamma \gamma, Z Z$, and $W^{+} W^{-}$. Our results from the Tevatron combined searches for the SM Higgs boson at $125 \mathrm{GeV}$, where much of the signal sensitivity is due to the fermionic decay mode $H \rightarrow b \bar{b}$, provides evidence for signal at the 3 s.d. level, offering complementary informaton on the nature of the Higgs boson observed at the LHC. Continuing to uncover the secrets that the discovered Higgs boson holds has begun a major and exiting theme of high energy physics. Whether or not the new particle is the Standard Model Higgs boson will be determined based on further investigations of its quantum properties and precise 
measurements of its coupling to quarks, leptons and gauge bosons. Any deviation from the SM prediction observed in the couplings of the Higgs boson to other fundamental particles will lead us to interesting routes for exploring new physics. 


\section{Bibliography}

[1] LEP Electroweak Working Group, http://lepewwg.web.cern.ch/LEPEWWG/.

[2] R. Barate et al., LEP Working Group for Higgs boson searches, ALEPH Collaboration, DELPHI Collaboration, L3 Collaboration, OPAL Collaboration, Phys.Lett. B565, 61 (2003), hep-ex/0306033.

[3] TEVNPH (Tevatron New Phenomena and Higgs Working Group), arXiv:1203.3774.

[4] T. Aaltonen et al., CDF Collaboration, D0 Collaboration, Phys.Rev. D82, 011102 (2010), 1005.3216.

[5] G. Aad et al., ATLAS Collaboration, Phys.Rev. D86, 032003 (2012), 1207.0319.

[6] S. Chatrchyan et al., CMS Collaboration, Phys.Lett. B710, 26 (2012), 1202.1488.

[7] G. Aad et al., (ATLAS Collaboration), Phys. Lett. B 716, 1 (2012).

[8] S. Chatrchyan et al., (CMS Collaboration), Phys. Lett. B 716, 30 (2012).

[9] T. Aaltonen et al., (CDF and D0 Collaborations), Phys. Rev. Lett. 109, 071804 (2012).

[10] V. M. Abazov et al., D0 Collaboration, Phys.Rev. D88, 052008 (2013), 1301.6122.

[11] V. M. Abazov et al., D0 Collaboration, Phys.Rev. D88, 052010 (2013), 1303.3276.

[12] V. M. Abazov et al., D0 Collaboration, Phys.Rev. D88, 052011 (2013), 1303.0823.

[13] T. Aaltonen et al., CDF Collaboration, D0 Collaboration, Phys.Rev. D88, 052014 (2013), 1303.6346.

[14] M. E. Peskin and D. V. Schroeder, An Introduction to Quantumn Field Theory, (Wesview Press).

[15] T. Lee and C.-N. Yang, Phys.Rev. 104, 254 (1956).

[16] C. S. Wu, E. Ambler, R. W. Hayward, D. D. Hoppes, and R. P. Hudson, Phys. Rev. 105, 1413 (1957). 
[17] K. Nakamura and P. D. Group, Journal of Physics G: Nuclear and Particle Physics 37, 075021 (2010).

[18] S. Dawson, p. 1 (1998), hep-ph/9901280.

[19] T. Hambye and K. Riesselmann, Phys.Rev. D55, 7255 (1997), hep-ph/9610272.

[20] G. Altarelli and G. Isidori, Phys.Lett. B337, 141 (1994).

[21] S. Schael et al., ALEPH Collaboration, DELPHI Collaboration, L3 Collaboration, OPAL Collaboration, SLD Collaboration, LEP Electroweak Working Group, SLD Electroweak Group, SLD Heavy Flavour Group, Phys.Rept. 427, 257 (2006), hepex/0509008.

[22] E. Braaten and J. Leveille, Phys.Rev. D22, 715 (1980).

[23] B. W. Lee, C. Quigg, and H. Thacker, Phys.Rev.Lett. 38, 883 (1977).

[24] W.-Y. Keung and W. J. Marciano, Phys.Rev. D30, 248 (1984).

[25] M. A. Shifman, A. Vainshtein, M. Voloshin, and V. I. Zakharov, Sov.J.Nucl.Phys. 30, 711 (1979).

[26] G. K. J. Gunion, H. Haber and S. Dawson, The Higgs Hunters Guide, (PERSEUS PUBLISHING).

[27] A. Djouadi, Phys.Rept. 457, 1 (2008), hep-ph/0503172.

[28] J. Beringer et al., Particle Data Group, Phys. Rev. D 86, 010001 (2012).

[29] Boughezal Radja, (2009), arXiv:0908.3641, Theoretical Status of Higgs Production at HadronColliders in the Standard Model.

[30] http://maltoni.home.cern.ch/maltoni/TeV4LHC/SM.html.

[31] T. Aaltonen et al., CDF Collaboration, D0 Collaboration, Phys.Rev.Lett. 109, 071804 (2012), 1207.6436.

[32] S. Chatrchyan et al., CMS Collaboration, Phys.Lett. B716, 30 (2012), 1207.7235.

[33] T. Aaltonen et al., CDF Collaboration, Phys.Rev.Lett. 108, 181804 (2012), 1201.4880.

[34] V. M. Abazov et al., D0 Collaboration, (2013), 1302.5723.

[35] V. Abazov et al., D0 Collaboration, (2012), 1211.6993.

[36] G. Aad et al., ATLAS Collaboration, Phys.Lett. B716, 1 (2012), 1207.7214.

[37] TEVNPH (Tevatron New Phenomina and Higgs Working Group), CDF Collaboration, D0 Collaboration, (2012), 1203.3774. 
[38] P. H. Frampton, P. Hung, and M. Sher, Phys.Rept. 330, 263 (2000), hep-ph/9903387.

[39] G. D. Kribs, T. Plehn, M. Spannowsky, and T. M. Tait, Phys.Rev. D76, 075016 (2007), 0706.3718.

[40] A. Akeroyd, Phys.Lett. B368, 89 (1996), hep-ph/9511347.

[41] E. Arik, O. Cakir, S. A. Cetin, and S. Sultansoy, Acta Phys. Polon. B37, 2839 (2006), hep-ph/0502050.

[42] C. Anastasiou, R. Boughezal, and E. Furlan, JHEP 06, 101 (2010), 1003.4677.

[43] S. Chatrchyan et al., CMS Collaboration, (2013), 1302.1764.

[44] G. Aad et al., ATLAS Collaboration, Eur.Phys.J. C71, 1728 (2011), 1106.2748.

[45] A. Akeroyd, Phys.Lett. B353, 519 (1995).

[46] A. Barroso, L. Brucher, and R. Santos, Phys.Rev. D60, 035005 (1999), hep$\mathrm{ph} / 9901293$.

[47] TMVA package (version 4.1.0), http://tmva.sourceforge.net/.

[48] L. Breiman, Mach. Learn. 45, 5 (2001).

[49] I. Narsky, (2005), physics/0507143.

[50] V. M. Abazov et al., D0 Collaboration, (2013), 1303.3276.

[51] Fermilab web picture book, http://www.fnal.gov/pub/about/whatis/picturebook/.

[52] R. Thurman-Keup, E. Lorman, T. Meyer, S. H. Pordes, and S. De Santis, Conf.Proc. C0505161, 2440 (2005).

[53] S. Abachi et al., D0 Collaboration, Nucl.Instrum.Meth. A338, 185 (1994).

[54] V. Abazov et al., D0 Collaboration, Nucl.Instrum.Meth. A565, 463 (2006), physics/0507191.

[55] M. Abolins et al., Nucl.Instrum.Meth. A584, 75 (2008), 0709.3750.

[56] R. Angstadt et al., D0 Collaboration, Nucl.Instrum.Meth. A622, 298 (2010), 0911.2522.

[57] R. Angstadt et al., Nuclear Instruments and Methods in Physics Research Section A: Accelerators, Spectrometers, Detectors and Associated Equipment 622, 298 (2010).

[58] Claus Grupen, Physics of particle detection, arXiv:physics/9906063, 2001.

[59] C. Fabjan and R. Wigmans, Rept.Prog.Phys. 52, 1519 (1989). 
[60] http://www d0.fnal.gov/computing/algorithms/howto/howtoreco.html.

[61] O. Atramentov, D. Bandurin, Y. Lin and Hang Yin, DØ Note 5635 (2008).

[62] T. H. Xuebing Bu and Y. Xie, Electron Identication for Summer 2010, DØ Note 6116, 2010.

[63] C. Fabjan and F. Gianotti, Rev.Mod.Phys. 75, 1243 (2003).

[64] J. Zhu et al., DØ Note 4323 (2003).

[65] V. M. Abazov et al., D0 Collaboration, Nucl.Instrum.Meth.A (2013), 1307.5202.

[66] U. Baur, R. K. Ellis, and D. Zeppenfeld, (2000).

[67] V. M. Abazov et al., (D0 Collaboration), Phys. Rev. D 85, 052006 (2012).

[68] N. Makovec and J. Grivaz, DØ Note 4914 (2005).

[69] C. Orchando and J. Grivaz, DØ Note 5609 (2008).

[70] V. Abazov et al., D0 Collaboration, Nucl.Instrum.Meth. A620, 490 (2010), 1002.4224.

[71] T. Sjöstrand, S. Mrenna, and P. Z. Skands, J. High Energy Phys. 05, 026 (2006).

[72] W.-K. Tung, Acta Phys.Polon. B33, 2933 (2002), hep-ph/0206114.

[73] F. Caravaglios, M. L. Mangano, M. Moretti, and R. Pittau, Nucl.Phys. B539, 215 (1999), hep-ph/9807570.

[74] E. Boos, V. Bunichev, L. Dudko, V. Savrin, and V. Sherstnev, Phys. Atom. Nucl. 69, 1317 (2006).

[75] John M. Campbell, R. Keith Ellis and Ciaran Williams, MCFM - Monte Carlo for FeMtobarn processes, http://mcfm.fnal.gov/.

[76] J. Alwall et al., Eur. Phys. J. C 53, 473 (2007).

[77] J. Baglio and A. Djouadi, J. High Energy Phys. 10, 064 (2010).

[78] D. de Florian and M. Grazzini, Phys. Lett. B 674, 291 (2009).

[79] P. Bolzoni, F. Maltoni, S.-O. Moch, and M. Zaro, Phys. Rev. D 85, 035002 (2012).

[80] M. L. Ciccolini, S. Dittmaier, and M. Kramer, Phys. Rev. D68, 073003 (2003), hep-ph/0306234.

[81] A. Djouadi, J. Kalinowski, and M. Spira, Comput. Phys. Commun. 108, 56 (1998).

[82] J. Butterworth et al., arXiv:1003.1643, (2010). 
[83] R. Hamberg, W. van Neerven, and T. Matsuura, Nucl. Phys. B359, 343 (1991), ibid, B644, 403 (2002).

[84] J. M. Campbell, arXiv:hep-ph/0105226, (2001).

[85] U. Langenfeld, S. Moch, and P. Uwer, Phys. Rev. D 80, 054009 (2009).

[86] N. Kidonakis, Phys. Rev. D 74, 114012 (2006).

[87] J. M. Campbell and R. K. Ellis, Phys. Rev. D 60, 113006 (1999).

[88] A. J. Emanuela Barberis and P. Schieferdecker, The Matrix Method and its Error Calculation, DØ Note 4564, 2004.

[89] D. H. Oleg Brandta, SungWoong Chob, Muon identication certication and treatment of systematic uncertainties for the Run IIb dataset, DØ Note 6326, 2012.

[90] M. Vesterinen, An Improved Energy Calibration and Monte Carlo Over-smearing for Electromagnetic Clusters Electron Identication for Summer 2010, DØ Note 6040, 2010.

[91] L. F. O. Brandt, D. Hedin and A. Santos, Muon Momentum Oversmearing Update for Run IIb1 and Run IIb2 Data Taking Epochs and Run IIb2 Monte Carlo, DØ Note $6314,2012$.

[92] G. F. Brendan Casey and M. Rangel, Jet ID/VC Eciencies and Scale Factors using Dijet events, DØ Note 6058, 2010.

[93] D. Boline, Jet Multiplicity Dependent Reweighting of the Z Boson pT in p20, DØ Note 5569, 2010.

[94] G. Hesketh, W pT Re-weighting for Alpgen and Pythi, DØ Note 5786, 2010.

[95] K. Melnikov and F. Petriello, Phys. Rev. D 74, 114017 (2006).

[96] V. M. Abazov et al., (D0 Collaboration), Phys. Lett. B 669, 278 (2008).

[97] V. M. Abazov et al., (D0 Collaboration), Phys. Rev. D 86, 032005 (2012).

[98] V. M. Abazov et al., (D0 Collaboration), Phys. Lett. B 698, 6 (2011).

[99] V. M. Abazov et al., (D0 Collaboration), Phys. Rev. Lett. 106, 171802 (2011).

[100] I. Narsky, arXiv:physics/0507143, (2005).

[101] I. Narsky, arXiv:physics/0507157, (2005).

[102] A. Hoecker et al., PoS ACAT, 040 (2007), we use version 4.1.0. 
[103] M. Buehler, B. Hirosky, S. Zelitch, and L. Zivkovic, Search for standard-model Higgs production in the lepton plus jets final state for $H \rightarrow W W^{*} \rightarrow \ell \nu j j$ decay in in 5.8 $\mathrm{fb}^{-1}$ of RunII data, DØ Note 005851, 2011.

[104] V. M. Abazov et al., D0 Collaboration, Phys.Rev.Lett. 109, 121803 (2012), 1207.5819.

[105] J. Beringer et al., Particle Data Group, Phys. Rev. D 86, 010001 (2012).

[106] S.B.Beri, X.Bu, S.Caughron, M.-C.Cousinou, S.Desai, S.Dutt, L.Han, P.Jiang, A.Kumar, Q.Li, E.Nagy, H.Nguyen, L.Welty-Rieger, T.Yasuda, J.Yu, Search for $Z H \rightarrow l l b b$ production in $9.7 \mathrm{fb}^{-1}$ of ppbar collisions, DØ Note 6342, 2012.

[107] T. Andeen et al., FERMILAB-TM-2365 (2007).

[108] W. Fisher et al., Studies of Alpgen parameters, corrections and associated uncertainties, D0 note 5966, 2009.

[109] T. Junk, Nucl. Instrum. Methods Phys. Res. A 434, 435 (1999).

[110] A. L. Read, J. Phys. G 28, 2693 (2002).

[111] W. Fisher, FERMILAB-TM-2386-E (2007).

[112] W. Fisher et al., Collie: A Confidence Level Limit Evaluator, D0 note 5595, 2010.

[113] P. Achard et al., L3 Collaboration, Phys. Lett. B517, 75 (2001), hep-ex/0107015. 